\title{
b-Tagging and the Search for Neutral Supersymmetric Higgs Bosons at $\mathrm{D} \emptyset$
}

\author{
Tim Scanlon \\ Imperial College London
}

A thesis submitted for the degree of

Doctor of Philosophy

of the University of London

and the Diploma of Imperial College

October, 2006 


\title{
b-Tagging and the Search for Neutral Supersymmetric Higgs Bosons at $\mathrm{D} \emptyset$
}

\author{
Tim Scanlon \\ Imperial College London
}

October, 2006

\begin{abstract}
A search for neutral supersymmetric Higgs bosons and work relating to the improvement of the $b$-tagging and trigger capabilities at the $\mathrm{D} \varnothing$ detector during Run II of the Fermilab Tevatron collider is presented. The search for evidence of the Higgs sector in the Standard Model (SM) and supersymmetric extensions of the $\mathrm{SM}$ are a high priority for the $\mathrm{D} \varnothing$ collaboration, and $b$-tagging and good triggers are a vital component of these searches.

The development and commissioning of the first triggers at $\mathrm{D} \varnothing$ which use $b$ tagging is outlined, along with the development of a new secondary vertex $b$-tagging tool for use in the Level 3 trigger. Upgrades to the Level 3 trigger hit finding code, which have led to significant improvements in the quality and efficiency of the tracking code, and by extension the $b$-tagging tools, are also presented.

An offline Neural Network (NN) b-tagging tool was developed, trained on Monte Carlo and extensively tested and measured on data. The new $b$-tagging tool significantly improves the $b$-tagging performance at $\mathrm{D} \varnothing$, for a fixed fake rate relative improvements in signal efficiency range from $\sim 40 \%$ to $\sim 15 \%$. Fake rates, for a fixed signal efficiency, are typically reduced to between a quarter and a third of their value.

Finally, three versions of the search for neutral supersymmetric Higgs bosons are presented. The latest version of the analysis makes use of almost $1 \mathrm{fb}^{-1}$ of data, the new NN $b$-tagger and the new $b$-tagging triggers, and has set one of the world's best limits on the supersymmetric parameter $\tan \beta$ in the mass range 90 to $150 \mathrm{GeV}$.
\end{abstract}




\section{Acknowledgements}

I would like to thank both my supervisors Gavin Davies and Per Jonsson for providing all their help, expertise and friendship, and for helping me out any trouble I may have gto into. I would also like to thank anyone else at Imperial who helped me with my thesis, and the HEP group for taking me on in the first place.

I would like to thank all the various people who convened groups I worked in for their help and leadership, and all the other people who helped me out at Fermilab. I would also like to thank Miruna and Stephen who both directly contributed to aspects of the work in my thesis.

I would also like to thank all my friends from London, Blackburn and Chicago. Without them it would have been a lot less fun and a lot harder to get through this $\mathrm{PhD}$.

Both my parents who have helped me throughout my whole life, and supported me whenever I needed it, and even when I didn't. Without them I wouldn't be here now.

Finally, and most importantly I would like to thank Maeve for putting up with me for the entire $\mathrm{PhD}$ and for always being there to help me get through it. Without her it wouldn't have been worth it. 


\section{Contents}

$\begin{array}{ll}\text { Abstract } & 2\end{array}$

$\begin{array}{ll}\text { Acknowledgements } & 3\end{array}$

$\begin{array}{ll}\text { Contents } & 4\end{array}$

$\begin{array}{ll}\text { List of Figures } & 9\end{array}$

List of Tables $\quad 22$

$\begin{array}{ll}\text { Preface } & 27\end{array}$

$\begin{array}{lll}\text { Chapter 1. Theory } & 30\end{array}$

1.1 The Standard Model 30

1.1.1 The Higgs Mechanism 33

1.1.2 Constraints on the Higgs 36

1.2 Limitations of the Standard Model and Supersymmetry 37

1.2.1 Higgs Sector of the MSSM 40

1.2.2 MSSM Higgs Searches 41

Chapter 2. The Tevatron and the DØ Detector 42

2.1 The Tevatron 42

2.2 The DØ Detector 42

2.2.1 The Central Tracking Detector 46

2.2.2 The Calorimeter 48

2.2.3 The Muon Detector 50 
2.2.4 Luminosity Monitor 50

2.2.5 The Trigger 51

2.2.6 Analysis Tools 53

$\begin{array}{lll}\text { Chapter 3. } & \text { Level } 3 \text { Trigger } & 57\end{array}$

3.1 Introduction $\quad 57$

$\begin{array}{lll}3.2 & \text { Tools } & 57\end{array}$

3.2.1 Unpacking Tools $\quad 57$

$\begin{array}{lll}3.2 .2 & \text { Tracking } & 57\end{array}$

$\begin{array}{lll}3.2 .3 & \text { Vertexing } & 59\end{array}$

$\begin{array}{lll}3.2 .4 & \text { Jets } & 59\end{array}$

3.2.5 Impact Parameter $b$-tagging Tool $\quad 59$

$\begin{array}{lll}3.2 .6 & \text { Muons } & 59\end{array}$

3.3 Tracking and SMT Studies 60

3.3.1 Measurement of the Tracking Performance 60

3.3.2 SMT Problems 61

$\begin{array}{lll}\text { 3.3.3 Results } & 68\end{array}$

$\begin{array}{lll}3.3 .4 & \text { Conclusion } & 71\end{array}$

3.4 SUSY Higgs Trigger Development 71

$\begin{array}{lll}3.4 .1 & \text { Introduction } & 71\end{array}$

3.4.2 Trigger Development 74

3.4.3 Trigger Performance 84

3.4.4 Online and Future Performance 84

3.4.5 Conclusion 86

3.5 Secondary Vertex Tagger 86

3.5.1 Vertexing Algorithm 87

$\begin{array}{ll}\text { 3.5.2 Data Optimisation } & 90\end{array}$

$\begin{array}{ll}\text { 3.5.3 Timing Studies } & 99\end{array}$

$\begin{array}{lll}3.5 .4 & \text { Conclusion } & 99\end{array}$ 
$\begin{array}{lll}\text { Chapter } 4 . & \text { The Neural Network } b \text {-Tagger } & 101\end{array}$

4.1 Introduction 101

4.1.1 Procedure for Data Certification 102

4.1.2 Neural Networks 105

$\begin{array}{lll}\text { 4.1.3 Data and MC Samples } & 107\end{array}$

4.2 Input Variables 108

$\begin{array}{lll}\text { 4.2.1 JLIP Variables } & 109\end{array}$

$\begin{array}{lll}4.2 .2 & \text { SVT } & 109\end{array}$

4.2.3 CSIP Variables 112

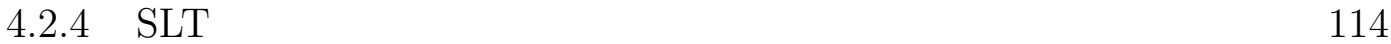

4.2.5 Primary Vertex 114

$\begin{array}{lll}4.2 .6 & \text { Jet Variables } & 114\end{array}$

4.3 Neural Network Optimisation 114

4.3.1 Training Samples 115

$\begin{array}{lll}\text { 4.3.2 Training Algorithms } & 115\end{array}$

$\begin{array}{lll}\text { 4.3.3 Performance Benchmark } & 115\end{array}$

4.3.4 Variable Optimisation 116

4.3.5 Number of Training Epochs 119

$\begin{array}{lll}\text { 4.3.6 Neural Network Structure } & 120\end{array}$

4.3.7 Input Selection Cuts 121

4.3.8 Optimised NN Parameters 122

4.4 Monte Carlo Performance 124

4.5 Data and Monte Carlo Variable Comparison 124

4.5.1 b-Jet Input Variables 124

4.5.2 Fake-Jet Input Variables 127

4.5.3 Data and Monte Carlo Neural Network Output Comparison 128

$\begin{array}{lll}4.6 & b-E f f i c i e n c y ~ M e a s u r e m e n t & 128\end{array}$

4.6.1 System8 Method 132

4.6.2 Measurement of the Correlation Coefficients 134

4.6.3 System8 Solution and Systematic Errors 136

4.6.4 Efficiency and Scale Factor Parameterisation 137 
4.6.5 Data/MC Scale Factor 138

4.6.6 Tag Rate Functions 138

4.6.7 Systematic Uncertainties 141

4.7 Fake Rate 146

$\begin{array}{lll}\text { 4.7.1 Negative Tag Rate } & 146\end{array}$

4.7.2 Negative Tag Rate Scale Factors 148

$\begin{array}{lll}\text { 4.7.3 Fake Rate } & 149\end{array}$

$\begin{array}{lll}\text { 4.7.4 Systematic Uncertainties } & 149\end{array}$

$\begin{array}{lll}4.8 & \text { Data Performance } & 155\end{array}$

4.9 Conclusion 156

Chapter 5. Search for Neutral Supersymmetric Higgs Bosons 159

$\begin{array}{llr}5.1 & \text { Introduction } & 159\end{array}$

5.1.1 Analysis Overview 161

5.1.2 The Three Analysis Generations 162

$\begin{array}{lll}5.2 & \text { Data } & 162\end{array}$

5.2.1 Skimming 164

5.2.2 Data Quality 164

5.3 Event Simulation 164

5.3.1 Signal Samples 166

$\begin{array}{lll}\text { 5.3.2 Background Samples } & 167\end{array}$

$\begin{array}{lll}5.4 & \text { Trigger } & 170\end{array}$

$\begin{array}{lll}\text { 5.4.1 Trigger Simulation } & 170\end{array}$

5.4.2 Trigger Efficiencies 171

5.4.3 Trigger Crosschecks 173

5.5 Pass1 Analysis 173

5.5.1 Event Selection 173

5.5.2 Analysis Method 174

$\begin{array}{lll}\text { 5.5.3 Limit Setting } & 178\end{array}$

5.5.4 Systematic Uncertainties 178

$\begin{array}{lll}\text { 5.5.5 Results } & 180\end{array}$

$\begin{array}{lll}5.6 & \text { Pass2 Analysis } & 182\end{array}$ 
5.6.1 Event Selection 182

5.6.2 Pass2 Analysis Problems 183

5.6.3 Neural Network Tagger Investigation 183

5.6.4 Background Composition Investigation 189

5.6.5 Pass2 Analysis Method 191

5.6.6 Systematic Uncertainties 192

5.6.7 Tagging Optimisation 194

$\begin{array}{lll}5.6 .8 & \text { Results } & 196\end{array}$

$\begin{array}{ll}5.6 .9 \text { Conclusions } & 199\end{array}$

5.7 p17 Analysis 199

$\begin{array}{lll}5.7 .1 & \text { Event Selection } & 199\end{array}$

$\begin{array}{lll}5.7 .2 & \text { Analysis Method } & 199\end{array}$

$\begin{array}{lll}\text { 5.7.3 Systematic Uncertainties } & 201\end{array}$

$\begin{array}{lll}5.7 .4 & \text { Results } & 202\end{array}$

5.8 Conclusion 204

Chapter 6. Conclusions and Outlook 205

$\begin{array}{lll}6.1 & \text { Summary } 205\end{array}$

6.2 Future Work 207

$\begin{array}{ll}\text { References } & 209\end{array}$ 


\section{List of Figures}

1.1 Graphical representation of $V(\phi)[9]$.

1.2 The ratio of the expected and observed 95\% CL limits to the SM cross section for the combined CDF and D $\varnothing$ analyses.

1.3 The $\Delta \chi^{2}\left(m_{H}\right)=\chi_{\text {min }}^{2}\left(m_{H}\right)-\chi_{\text {min }}^{2}$ of the fit to the EW parameters and the Higgs mass as a function of the Higgs mass [16]. The band represents the theoretical uncertainty, and the vertical band shows the 95\% confidence level exclusion limit found from the direct searches at LEP. 38

1.4 The theoretical limits on the mass of the Higgs $\left(M_{H}\right)$ as a function of $\Lambda$, the energy where new physics appears [17].

1.5 The running values of the inverse of the coupling constants as a function of the energy scale, Q, in the SM (left) and the MSSM (right) [4], where $\alpha_{1}, \alpha_{2}$ and $\alpha_{3}$ are the $\mathrm{U}(1), \mathrm{SU}(2)$ and $\mathrm{SU}(3)$ coupling constants respectively.

2.1 The system of accelerators at the Fermi National Accelerator Laboratory used to produce and accelerate protons and antiprotons.

2.2 The peak instantaneous luminosity delivered by the Tevatron during Run IIa $[28]$.

2.3 A cross section of the $\mathrm{D} \varnothing$ detector. The central tracking region is shown in more detail in Fig. 2.4.

2.4 The central tracking region in the DØ detector. 44

2.5 The data-taking efficiency of the D $\varnothing$ detector during Run IIa [31]. 45

2.6 The silicon microstrip tracker. $\quad 47$

$\begin{array}{lll}2.7 & \text { The central and two end cap calorimeters. } & 48\end{array}$ 
2.8 The $\mathrm{D} \varnothing$ trigger and data acquisition systems.

2.9 The different characteristics used to identify b-jets: a displaced secondary vertex, displaced tracks with large impact parameters and high $\mathrm{p}_{T}$ leptons. 56

3.1 For data run 189917 the number of axial (left) and stereo (right) SMT clusters assigned to the L3 (green) and offline (red) tracking tools. The distributions were normalised to be of equal integral.

3.2 For data run 189917 the number of axial clusters (left) and stereo clusters (right) found by the L3 (green) and offline (red) SMT unpacking tools.

3.3 The number of hit strips in each SVX chip (left) and on a log scale (right) for 2500 events from data run 189917. Each SVX chip reads out 128 strips. 63

3.4 The number of clusters per event found due to 'hot' SVX chips.

3.5 An example from a data event of consecutive strips in the SMT detector, all of which registered a hit except strip 2 which is a 'dead' or 'noisy' strip. 65

3.6 The number of merged clusters which contain a 'dead' or 'noisy' strip per event, based on 2500 events.

3.7 An example from a data event of consecutive strips in the SMT detector which are divided by the splitting algorithm. Three distinct peaks are found at strips $2,5+6$ and $8+9$, and two troughs are found at $3+4$ and 7 . The large cluster is therefore split into 3 smaller clusters corresponding to the three peaks. The strips in the troughs are split amongst the clusters. The first cluster gets strip 3 and the second strip 4 . The second and third clusters both get half of strip 7 .

3.8 The number of clusters per event created by cluster splitting (left) and the number of clusters each split cluster produces (right) based on 2500 events.

3.9 The residuals between the L3 tracks and the closest L3 cluster for the axial layers (left) and the stereo layers (right). The tracks were all required to have 8 CFT and at least 2 SMT hits matched to an offline track.

3.10 The number of axial clusters (top) and stereo clusters (bottom) per event found by the old L3 (blue), offline (red) and new L3 (green) SMT unpacking tools. 
3.11 The track efficiency (left) and purity (right) found with the old L3 (red) and new L3 (green) SMT unpacking tools.

3.12 The number of axial (left) and stereo (right) SMT clusters associated with 'good' matched tracks (at least 8 CFT and 2 SMT clusters matched with an offline track) found using the old L3 (green) and new L3 (red) SMT unpacking tools.

3.13 The timing distributions, in milliseconds, for the SMT unpacking tool (left) and the tracking tool (right) over 2500 events, when using the old (red) and new (green) SMT unpacking tools.

3.14 Left: The L3 IP $b$-tag distribution for the $h b$ signal sample (green) and the background (red). Right: The signal (green circles) and background (red squares) efficiencies as a function of the L3 IP b-tag cut. The black dotted lines are at a cut value of 0.05 .

3.15 Left: The $1^{\text {st }}$ (top), $2^{\text {nd }}$ (middle) and $3^{\text {rd }}$ (bottom) leading jet $E_{T}$ for the $h b$ signal sample (green) and the background (red). Right: The trigger efficiencies for signal (green circles) and background (red squares) as a function of the jet $E_{T}$ cut on the $1^{\text {st }}$ (top), $2^{\text {nd }}$ (middle) and $3^{\text {rd }}$ (bottom) leading jets after the L1L2 cuts and a L3 $b$-tag IP cut $<0.05$. The black dotted lines are at the v12 cut values.

3.16 Left: The L3 Loose muon $\mathrm{p}_{T}$ distribution in the $h b$ signal sample (green) and the background (red), before (top) and after (bottom) requiring a Loose L3 muon. Right: The signal (green circles) and background (red squares) efficiencies as a function of the L3 muon $\mathrm{p}_{T}$ cut before (top) and after (bottom) requiring a Loose L3 muon. The black dotted lines are at a cut value of $3.0 \mathrm{GeV}$.

3.17 Left: The $1^{\text {st }}$ (top), $2^{\text {nd }}$ (middle) and $3^{\text {rd }}$ (bottom) leading jet $E_{T}$ distributions for the $h b$ signal sample (green) and the background (red). Right: The trigger efficiencies for signal (green circles) and background (red squares) as a function of the jet $E_{T}$ cut on the $1^{\text {st }}$ (top), $2^{\text {nd }}$ (middle) and $3^{\text {rd }}$ (bottom) leading jets, after the L1L2 cuts and a Loose L3 Muon with $p_{T}>3 \mathrm{GeV}$. The black dotted lines are at the v12 cut values. 
3.18 The L3 track IP (IP) significancies for all events (left) and for those with at least one offline JLIP tag (right) in a $b$-enriched 'special run'. The fit is a double exponential and a gaussian.

3.19 The L3 IP $b$-tagging tool output for jets which pass a JLIP probability cut of 0.003 (blue line), 0.01 (green line) and 0.02 (red line). The spike at one corresponds to jets with too few tracks at L3 with which to calculate a $b$-tag.

3.20 Left: The L3 IP $b$-tagging tool's efficiency to tag jets which have a JLIP cut of 0.003 (blue line), 0.01 (green line) and 0.02 (red line). Right: The L3 IP $b$-tagging tool's efficiency to tag events which have three jets with JLIP cuts of 0.003 (blue line), 0.01 (green line) and 0.02 (red line).

3.21 The L1L2L3 signal efficiency of various trigger cuts versus the rate to tape at a luminosity of $60 \times 10^{30} \mathrm{~cm}^{-2} \mathrm{~s}^{-1}$. Each line represents a cut on a L3 tool and the points along the line represent the different cut values. The black star is the final trigger.

3.22 The L1L2L3 signal efficiency of various trigger cuts versus the rate to tape at a luminosity of $60 \times 10^{30} \mathrm{~cm}^{-2} \mathrm{~s}^{-1}$. Each line represents a cut on a L3 tool and the points along the line represent the different cut values. The black star is the final trigger.

3.23 A single track in a jet which is linearised at the DCA to the EP (jx, jy). The vertex position $(\mathrm{V})$ is found by minimising the $d_{0}$ of all the tracks, with an additional constraint from $\sigma_{j}$.

3.24 The distance from the jet axis $(\mathrm{dR})$ as a function of distance along the jet axis (dj) for offline 'Tight' SVs (top left). The distribution of SVs around the jet axis is characterised by fitting gaussian functions to slices along the jet axis. The three gaussian fit parameters, the constant (top right), mean (bottom left) and sigma (bottom right), are shown as a function of the distance along the jet axis.

3.25 The $\chi_{\text {dof }}^{2}$ as a function of distance along the jet axis (dj) for a 'typical' data event. Values of dj with no $\chi_{\text {dof }}^{2}$ value occur due to there being no SVs reconstructed due to a lack of tracks. 
3.26 The performance of the L3 SVT $b$-tagging tool as a function of the iterative $\chi^{2}$ threshold. A representative sample of thresholds of 0.1 (red circles), 0.5 (green squares), 1.0 (blue triangles) and no iteration (purple diamonds) are shown. Errors shown are statistical.

3.27 The performance of the L3 SVT b-tagging tool as a function of the split vertex threshold, $\chi_{\text {split }}^{2}$. A representative sample of thresholds of 0.0 (red circles), 0.5 (green squares), 2.0 (blue triangles) and 8.0 (purple diamonds) are shown. Errors shown are statistical.

3.28 The performance of the L3 SVT $b$-tagging tool for various values of the threshold $S_{\text {Track. }}^{P V}$. The thresholds are 0 (red circles), 1 (green squares), 2 (blue triangle), 3 (purple diamond), 4 (cyan cross) and 5 (grey star). Errors shown are statistical.

3.29 The performance of the L3 SVT $b$-tagging tool for various values of the threshold $S_{\text {Track }}^{S V}$. The thresholds are 4 (red circles), 6 (green squares), 8 (blue triangles) and no threshold (purple diamonds). Errors shown are statistical.

3.30 The performance of the L3 SVT $b$-tagging tool for various track $\chi_{d o f}^{2}$ thresholds. The lines represent the thresholds 1.0 (red circles), 2.0 (green squares), 2.5 (blue triangles) and 5.0 (purple diamonds). Errors shown are statistical.

3.31 The performance of the L3 SVT b-tagging tool for various track $\mathrm{p}_{T}$ thresholds. The lines represent the thresholds 0.5 (red circles), 1.0 (green squares) and $1.5 \mathrm{GeV}$ (blue triangles). Errors shown are statistical.

3.32 The performance of the optimised L3 SVT $b$-tagging tool for the six operating points: PV0 (red circles), PV1 (green squares), PV2 (blue triangles), PV3 (purple diamonds), PV4 (cyan crosses) and PV5 (grey stars). Errors shown are statistical.

3.33 The DLS (top left), $-\log \left(\chi_{\text {dof }}^{2}\right)$ (top right), decay length (middle left), $N_{\text {Tracks }}$ (middle right) and the number of vertices (bottom) for the signal sample (red line) and background sample (green line) for the operating point PV0. 
3.34 The timing distributions for the L3 SVT $b$-tagging tool operating point PV0 on the signal events (red line) and background events (green line).

3.35 The time taken as a function of the number of tracks in the signal events for the operating point PV0.

4.1 The two methods used to apply $b$-tagging to $\mathrm{MC}$ and data.

4.2 JLIP tagger variables Prob (top left), $-\log _{10}$ (Prob) (top right), $\operatorname{Prob}_{\text {Red }}$ (bottom left) and the $N_{\text {Tracks }}$ (bottom right) for QCD $b \bar{b}$ (red) and fake (green) MC jets. See the text for the full definitions of variables.

4.3 Variables for the Loose SVT tagger, DLS (top left and right), $\chi_{d o f}^{2}$ (middle left), $N_{\text {Tracks }}$ (middle right), Mass (bottom left) and number of vertices (bottom right) for QCD $b \bar{b}$ (red) and fake (green) MC jets. See the text for a full description of the variables.

4.4 $\mathrm{SVT}_{S L}$ (red circles) and $\mathrm{SVT}_{L}$ (green squares) efficiencies for QCD $b \bar{b}$ and QCD fake MC jets.

4.5 Variables from the Loose CSIP tagger, the number of tracks with a significance $\geq 2$ (top left), the number of tracks with a significance $\geq 3$ (top right) and the combination variable (bottom) for QCD $b \bar{b}$ (red) and fake (green) MC jets. See the text for a full description of the variables.

4.6 The $\mathrm{p}_{T}$ (left) and the $\eta$ (right) of the training and test samples.

4.7 Training curves for the various training algorithms for a fixed amount of CPU time.

4.8 Fake rate for fixed signal efficiencies of $75 \%$ (top), and $50 \%$ (bottom) as a function of additional NN variables. The NN variables were added to the $\mathrm{NN}$ in order of performance. The fit is intended to guide the eye only. The errors are statistical only.

4.9 Performance curves for the 5 (red circles), 7 (green squares) and 9 (blue triangles) variable NN. The 7 and 9 variable NNs have better performance than the 5 variable NN, although there is no improvement in going from 7 to 9 variables. 
4.10 Fake rate for fixed signal efficiencies of $75 \%$ (left) and $50 \%$ (right) as a function of the number of training epochs. The majority of the minimisation is achieved by 200 epochs for each of the operating points with only small increases in performance thereafter. The fit is intended to guide the eye only.

4.11 Fake rate for fixed signal efficiencies of $75 \%$ (left) and $50 \%$ (right) as a function of the number of hidden nodes in a single hidden layer. There is a minimum at 24 hidden nodes for each of the operating points. The fits are designed to guide the eye only.

4.12 The NN output for MC QCD $b \bar{b}$ (red) and fake-jets (green) for the case of a single hidden layer 7:24:1 (left) and double hidden layer 7:24:1:1 (right). The extra hidden layer constrains the NN between 0 and 1 .

4.13 Fake rate for fixed signal efficiencies of $75 \%$ (left) and $50 \%$ (right) as a function of the $\mathrm{SVT}_{S L}$ DLS cut (top), JLIP Prob cut (middle) and CSIP Comb cut (bottom) on the input jets. The fits are designed to guide the eye only.

4.14 NN output for QCD MC $b$ (green) and fake-jet (red) samples, after the $\mathrm{NN}$ jet input selection cuts.

4.15 Performance curves for the NN tagger (red circles) and the JLIP tagger (green squares) for QCD $b$ and $b$-like fake-jet samples.

4.16 Tag rates for the JLIP (green squares), CSIP (purple triangles), SVT (blue triangles) and NN (red circles) taggers on QCD $b$ (top) and fakejet (bottom) samples in $\mathrm{p}_{T}$ (left) and $\eta$ (right) projections.

4.17 NN input variables in QCD $b \bar{b}$ (red line) MC and the $b$-enriched data sample (green squares) after the NN jet input cuts. The variables are JLIP Prob (top left), $-\log _{10}$ (JLIP Prob)(top right), CSIP Comb (2nd left), $\mathrm{SVT}_{L} N_{\text {Tracks }}$ (2nd right), $\mathrm{SVT}_{S L}$ DLS (3rd left), $\mathrm{SVT}_{S L} \chi_{d o f}^{2}$ (3rd right), $\mathrm{SVT}_{S L}$ Mass (bottom left) and $\mathrm{SVT}_{S L}$ (bottom right). Comparisons should be made between the shape and not the absolute levels of the input variables, as their areas have been normalised to be equal. 
4.18 NN input NT variables in QCD MC (red line) and data (green squares) after the NN jet input cuts. The variables are JLIP Prob (top left), $-\log _{10}$ (JLIP Prob) (top right), CSIP Comb (2nd left), $\mathrm{SVT}_{L} N_{\text {Tracks }}$ (2nd right), $\mathrm{SVT}_{S L}$ DLS (3rd left), $\mathrm{SVT}_{S L} \chi_{d o f}^{2}$ (3rd right), $\mathrm{SVT}_{S L}$ Mass (bottom left) and $\mathrm{SVT}_{S L}$ (bottom right). Comparisons should be made between the shape and not the absolute levels of the input variables, as their areas have been normalised to be equal.

4.19 NN output data/MC comparison: Top - The b-enriched skim (red) and QCD $b \bar{b}$ MC (green). Bottom - The NT outputs from the QCD (green) and EM (red) data skims compared to the QCD MC sample (blue). Comparisons should be made between the shape and not the absolute levels of the NN outputs, as their areas have been normalised to be equal.

4.20 The process to measure the TRFs for inclusive $b$ and $c$-jets.

4.21 Top: The Loose NN tagging efficiency on muonic $b$-jets (green squares), the tagging efficiency on muonic $b$-jets with an away tag (red squares), and their ratio, $\beta$ (blue line) in the $b \rightarrow \mu \mathrm{MC}$ sample as a function of jet $p_{T}$ (left) and $\eta$ (right). The fit error on $\beta$ is represented by two black dotted lines although the error is to small for these to be seen. Bottom: The ratio between $\beta$ found in the $Z \rightarrow b \bar{b} \rightarrow \mu$ and $t \bar{t} \rightarrow b \rightarrow \mu$ samples in $\mathrm{p}_{T}$ (left) and $\eta$ (right) projections.

4.22 Top: The efficiency of the SLT tagger (red circles), the Loose NN tagger (green squares), the AND of the NN and SLT taggers (blue triangles), and $\kappa_{b}$ (upside down purple triangles), measured in the $b \rightarrow \mu \mathrm{MC}$ sample in the jet $p_{T}$ (left) and $\eta$ (right) projections. The black dotted line represents the error on the fit calculated from the full covariant fit error matrix. Bottom: The ratio of $\kappa_{b}$ measured in the $Z \rightarrow b \bar{b} \rightarrow \mu$ and $t \bar{t} \rightarrow b \rightarrow \mu \mathrm{MC}$ samples in $\mathrm{p}_{T}$ (left) and $\eta$ (right).

4.23 The Loose NN tagger scale factor (blue line) and the data (green squares) and $\mathrm{MC}$ (red circles) b-jet efficiencies as a function of $\mathrm{p}_{T}$ (top) and $\eta$ (bottom). The functions used for the parameterisation are outlined in the text and the black dotted curves represent the $\pm 1 \sigma$ statistical error. 
4.24 The $\alpha$ (red), $\beta$ (green), $\kappa_{b}$ (blue), $\kappa_{u d s c}$ (purple), $P_{T R e l}$ (cyan) and total combined (empty grey squares) S8 relative systematic errors calculated for each bin in $\mathrm{p}_{T}$ (left) and $\eta$ (right) for the Loose operating point. The grey line is a constant fit to the total combined error.

4.25 NN tagger inclusive $b$-jet efficiency (top) and $c$-jet efficiency (bottom) as a function of $\mathrm{p}_{T}$ (left) and $\eta$ (right) in both data (green line) and MC (red circles). The data TRF is calculated by multiplying the MC TRF by the data/MC SF. The dotted black lines represent the error on the fit which is almost entirely inherited from the error on the scale factor. The functions used for the parameterisation are outlined in the text.

4.26 The fractional differences between the actual and predicted tags calculated in $\mathrm{p}_{T}$ bins in the CC (green), ICR (blue), EC (purple) and all (red) for the $t \bar{t} \rightarrow b$ (top) and the $b$ sample (bottom). The Mean (M) and root mean square (RMS) of the distributions are in the legend. The fractional errors are weighted by the number of actual tags in each bin and the RMS is used to estimate the error on the $b$-jet TRF.

4.27 The total relative error (combined systematic and statistical) for the Scale Factor (SF) (top), $\mathrm{TRF}_{b}$ (middle) and $\mathrm{TRF}_{c}$ (bottom) for the L4 (red circles), L3 (green squares), L2 (blue triangles), Loose (purple triangles), Medium (cyan empty circles) and Tight (grey empty squares) NN operating points in terms of $\mathrm{p}_{T}$ (left) when $\eta=1.2$ and $\eta$ (right) when $p_{T}=45 \mathrm{GeV}$.

4.28 The process used to measure the fake rate in the DØcertification.

4.29 The NTR parameterisation for the COMB skim in the CC (red circles), ICR (green squares) and EC (blue triangles). The NTR is parameterised with a second order polynomial and the dotted lines show the $\pm 1 \sigma$ fit error.

4.30 The heavy flavour correction, $S F_{h f}$ (red circles), light jet asymmetry correction, $S F_{l l}$ (green squares), and total NT SF correction (blue triangles) in the $\mathrm{CC}$ (top left), ICR (top right) and EC regions (bottom) for the L4 operating point. For fit details see the text. 
4.31 The FTRs parameterised in the CC (red), ICR (green) and EC (blue)for the Loose operating point. The dotted black lines represent the fit error. 150

4.32 The NTR parameterisation for the EM skim (top left) and the QCD skim (top right) in the CC (red circles), ICR (green squares) and EC (blue triangles). The NTR is parameterised with a second order polynomial and the dotted lines show the $\pm 1 \sigma$ fit error. The ratio of the EM and QCD NTRs in the CC (middle left), ICR (middle right) and EC (bottom) regions is also shown.

4.33 The predicted and actual number of NTs in the three eta regions for the Loose tagger on the COMB skim (top) and the relative difference between the actual and predicted number of NTs in the CC (middle left), ICR (middle right) and EC (bottom) $\eta$ regions.

4.34 The total relative error (combined systematic and statistical) for the L4 (red circles), L3 (green squares), L2 (blue triangles), Loose (purple triangles), Medium (cyan empty circles) and Tight (grey empty squares) NN operating points on the Fake-Tag Rate in the CC (top left), ICR (top right) and $\mathrm{EC}$ (bottom) as a function of $\mathrm{p}_{T}$. The error on the L4 operating point in the $\mathrm{CC}$ increases at large $\mathrm{p}_{T}$ due to the uncertainty in the NTSF fits.

4.35 Performance profile of the NN (blue circles) and JLIP (green squares) taggers on the $b$-ID and COMB skim. The errors represent the total uncertainty, statistical and systematic. The NN tagger shows large performance gains over the JLIP tagger, with relative increases in efficiency of up to $50 \%$ for a fixed fake rate. Fake rates are typically reduced to between a quarter and a third of their value for a fixed signal efficiency.

4.36 'Data' performance profile of the NN (blue circles) and JLIP (green squares) taggers on $Z \rightarrow b \bar{b}$ and $Z \rightarrow q \bar{q}$ MC samples. The performance is calculated on a jet by jet basis. The error represents the full statistical and systematic error.

5.1 The production cross section [88] for the SM Higgs Boson at the Tevatron.159 5.2 The branching ratio [88] for the SM Higgs Boson. 
5.3 Leading-Order Feynman diagrams showing gb $\rightarrow$ hb and gg, $q \bar{q} \rightarrow b \bar{b}$ h production.

5.4 The $h b$ analysis triple $b$-tagged background prediction method. The different coloured boxes correspond to: data samples (blue), derived samples (green) and processes (red).

5.5 The $\mathrm{p}_{T}$ (left) and $\eta$ distribution (right) of the jets in the Pass1 (top), Pass2 (middle) and p17 data sets from the highest $\mathrm{p}_{T}$ (1st jet) to the lowest $\mathrm{p}_{T}$ jet (4th or 5th). The jets have passed the skimming, trigger, data quality and taggability requirements.

$5.6 \mathrm{p}_{T}$ (left) and $\eta$ distribution (right) for the Pass2 MC samples from the highest $\mathrm{p}_{T}$ (1st jet) to the lowest $\mathrm{p}_{T}$ jet (5th). The jets have passed the skimming, trigger, quality and taggability requirements.

5.7 Production cross sections for neutral Higgs bosons associated with a $b \bar{b}$ pair at leading-order as a function of $m_{A}$ only, for $\tan \beta$ of 30 calculated using HQQ [88]. The A is solid, the $\mathrm{h}$ is dashed, and the $\mathrm{H}$ is dotted. The sum of $\mathrm{h}, \mathrm{H}$, and A production is shown dashed-dotted, and is always equal to twice the A production alone.

5.8 Higgs width as a function of mass and $\tan \beta$. At high $\tan \beta$ and mass the width is larger than the experimental resolution of $\sim 20 \mathrm{GeV}$.

5.9 The total simulated double $b$-tagged di-jet mass distribution (black line), consisting of $j j j$ (red dotted line), bbj (blue dotted line), and other small backgrounds: $Z j, t t$, and $b b b b$ (Purple dot-dashed line), to data (black dots). The $j j j$ distribution shown in this figure has a heavy flavour component which is corrected for, as explained in the text.

5.10 $\mathrm{TRF}_{\text {all }}$ (red points) and FTRF (black points).

5.11 Fit of the total background (black line) consisting of $b b j$ (blue dotted line), jjj (red dotted line) and other small backgrounds (purple dotdashed line) to the double tagged data (black points) sample using the corrected FTRF. 
5.12 The MC predicted triple tagged background (black line) for the Loose SVT tagger, consisting of the correctly normalised MC bbj sample (blue dotted line), the $j j j$ prediction from data (red dotted line) and other small MC contributions (purple dot-dashed line), compared to the triple tagged data sample (black dots).

5.13 The data (points), normalised background (solid line), and the Higgs signal for $m_{A}=120 \mathrm{GeV}$ (dashed line) at $\tan \beta \simeq 100$.

5.14 The observed $95 \%$ C.L. limits on $\tan \beta$ as a function of $m_{A}$ (area above blue line) and overlayed exclusion limits from the p13 analysis [23] (area above red dotted line), assuming $\tan ^{2} \beta$ cross section enhancement (treelevel results for the MSSM).

5.15 The Pass2 double (left) and triple tag (right) di-jet mass distributions for the Loose SVT tagger (top), Tight SVT tagger (middle) and Tight NN tagger (bottom). Data (black points), predicted background (black line) and MC predicted background (dotted black line) are all shown.

5.16 The fake-tag rates in di-jet (red circles) and multi-jet events (green squares) for a representative NN operating point (Medium). The fake-tag rates are in agreement within errors.

5.17 NN input variables in the QCD di-jet data sample (green square) and the 3JET multi-jet data sample (red circle) after the NN jet input cuts. The variables are JLIP Prob (top left), $-\log _{10}$ (JLIP Prob)(top right), CSIP Comb (2nd left), $S V T_{L} N_{\text {Tracks }}$ (2nd right), $S V T_{S L}$ DLS (3rd left), $S V T_{S L} \chi_{d o f}^{2}$ (3rd right), $S V T_{S L}$ Mass (bottom left) and $S V T_{S L}$ (bottom right). The input events are normalised to have equal number.

5.18 NN input variables in the QCD di-jet data sample (green square) and the 3JET multi-jet data sample (red circle) after the NN jet input cuts and 1 tag required in the event. The variables are JLIP Prob (top left), $-\log _{10}$ (JLIP Prob)(top right), CSIP Comb (2nd left), $S V T_{L} N_{\text {Tracks }}$ (2nd right), $S V T_{S L}$ DLS (3rd left), $S V T_{S L} \chi_{d o f}^{2}$ (3rd right), $S V T_{S L}$ Mass (bottom left) and $S V T_{S L}$ (bottom right). The input events are normalised to have equal number. 
5.19 The TRFs for all jets in the sample, $\mathrm{TRF}_{\text {all }}$ (red dots), and for jets after 2 tags have been applied, $\mathrm{TRF}_{2 t a g}$ (green squares), in the eta regions $\eta<1.1$ (top), $1.1<\eta<1.5$ (middle) and $1.5<\eta<2.5$ (bottom).

5.20 The Pass2 double (left) and triple tag (right) di-jet mass distributions for the Loose (top), Medium (middle) and Tight (bottom) NN taggers. Data (black points), predicted background (black line) and MC predicted background (dotted black line) are all shown. A good agreement exists between the data and MC predicted backgrounds for all operating points. 192

5.21 Sensitivity as a function of operating point for the NN (blue dots) and SVT (red squares) taggers for $\tan (\beta)=50$. For the cases of: no systematic error on the background prediction (top) and full systematic errors (bottom).

5.22 Expected limits for the NN and SVT taggers: no systematic errors on the background prediction (top), the Pass 2 systematic errors on the background prediction (middle) and the Pass1 systematic errors for the Pass1 and Pass2 SVT taggers (bottom).

5.23 Data MC comparison of the di-jet invariant mass distributions in the double $b$-tag sample (top) and the triple $b$-tag sample (bottom).

5.24 The observed and expected $95 \%$ confidence limits on $\tan \beta$ as a function of $m_{A}$, assuming $(\tan \beta)^{2}$ cross section enhancement. The error bands indicate the $\pm 1 \sigma$ and $\pm 2 \sigma$ ranges on the expected limit.

5.25 The di-jet invariant mass distribution for the data (circles), signal (solid grey line), background (solid black line) and combined signal and background (dashed black line) for $m_{A}=120 \mathrm{GeV}$ at the observed $95 \%$ confidence limit $(\tan \beta=60)$. 


\section{List of Tables}

2.1 The Tevatron operating parameters in Run I and II [30]. 45

2.2 Approximate trigger rates and latency for the three trigger levels. $\quad 51$

3.1 The threshold cuts used in the old L3, new L3 and offline SMT unpacking tools.

3.2 The errors assigned to the SMT hits in the new and old versions of the L3 SMT unpacking tool.

3.3 The times measured for each component of the tracking before and after the upgrade of the L3 SMT unpacking tool. Errors on timing tests were estimated to be $\sim 10 \%$ from repeated measurements.

3.4 Efficiency (\%) of the v12 and v13 triggers on events which have a minimum of 3 offline jets with $E_{T}>8 \mathrm{GeV}$, measured on the $h b\left(m_{h}=100 \mathrm{GeV}\right)$ $\mathrm{MC}$ sample. The $\mathrm{OR}$ of the triggers measures the joint efficiency of the two triggers. The errors contain both a statistical and systematic $(\sim 5.5 \%[71])$ component.

3.5 The rates for the two v13 $h b$ triggers for an average instantaneous luminosity of $40 \times 10^{30} \mathrm{~cm}^{-2} \mathrm{~s}^{-1}$ measured on run $207292,60 \times 10^{30} \mathrm{~cm}^{-2} \mathrm{~s}^{-1}$ measured on run 206944 and $80 \times 10^{30} \mathrm{~cm}^{-2} \mathrm{~s}^{-1}$ measured on run 206692 . The rates for trigger JT3_3JT10L_LM3_V in run 206944 are corrected to account for a pre-scale of 5 . The average rates over the range of instantaneous luminosities are within the design goals.

3.6 The final optimised thresholds used in the L3 SVT b-tagging tool

3.7 Representative signal efficiencies and rates for the six operating points defined for the L3 SVT b-tagging tool. 
3.8 The time taken by the L3 SVT $b$-tagging tool, and the tracking and vertexing tools for comparison, in the signal and background samples. Errors on the times were estimated to be $\sim 10 \%$ from repeated measurements.

4.1 The NN tagger's OPs with their corresponding design fake rates.

4.2 Calorimeter regions (which have different detector responses) in the D $\varnothing$ detector.

4.3 Data skims used in the certification of the NN tagger.

4.4 MC samples used in the development and certification of the NN tagger. All the samples were generated using Pythia [70], except for the $t \bar{t}$ sample which was generated using Alpgen [85] before being processed through Pythia to simulate the hadronisation and showering.

4.5 Number of MC jets of each flavour available in each of the combined and merged samples after data processing and jet selection.

4.6 The track selection cuts used for the standard SVT operating points and the Super Loose SVT tagger. The tracks are selected based upon their $\chi^{2}$, impact parameter significance $\left(I P_{\text {sig }}\right)$, transverse momentum $\left(\mathrm{p}_{T}\right)$ and number of SMT hits.

4.7 NN input variables ranked in order of power.

4.8 Optimised NN parameters.

4.9 Away tag cut applied to the $b$-ID skim. Designed to primarily reduce the $c$-jet content in the $b$-enriched sample.

4.10 The QCD content (estimated from QCD MC) along with the SLT and away tag efficiencies used to estimate the flavour content in the $b$-enriched sample.

4.11 The S8 correlation coefficient values for the Loose operating point measured in the $\mathrm{p}_{T}$ and $\eta$ projections. $\kappa_{u d s c}$ is evaluated at $\mathrm{p}_{T}=39 \mathrm{GeV}$ in the $\mathrm{p}_{T}$ projection (the average $\mathrm{p}_{T}$ of the sample). The values measured in the $\mathrm{p}_{T}$ and $\eta$ projections are consistent within errors.

4.12 The $b$-efficiencies and their relative systematic uncertainties measured on the $b$-ID skim. The total systematic uncertainty was determined by adding the individual uncertainties in quadrature. 
4.13 The $b$-jet TRF error for the $b$-jet sample and each of the constituent MC samples. The sample with the largest error is used as the error on the TRF.

4.14 The muonic $b$-jet TRF error for the muonic $b$-jet sample and each of the constituent MC samples. The sample with the largest error is used as the error on the TRF.

4.15 The $c$-jet TRF error for the $c$-jet sample and each of the constituent MC samples. The sample with the largest error is used as the error on the TRF.

4.16 Total systematic errors on the MC sample parameterisations, the SF and the TRFs.

4.17 NTR measured for the COMB skim, for all $\mathrm{p}_{T}$ in the three calorimeter regions.

4.18 The data FTR calculated on the COMB skim, in the three calorimeter regions for all $\mathrm{p}_{T}$.

4.19 Errors assigned to the difference in the NTRs for the EM and QCD skims..152

4.20 Errors assigned to the difference in the NTRs for the EM and QCD skims for jets with $\mathrm{EMF}<0.8$.

4.21 The systematic uncertainties on the COMB skim parameterisation.

4.22 The systematic error in the FTR due to the uncertainty in the $b$-content in the MC.

4.23 The systematic error in the FTR due to the uncertainty in the $c$-content in the MC.

4.24 Total systematic errors on the FTR.

5.1 The percentage of runs in the different trigger list epochs which are classed as bad in the SMT, CFT, Muon, Cal and Any detector subsystems [90]. The 'All pre-v13' epoch corresponds to the p14 analyses and the 'All' epoch corresponds to the p17 analysis.

5.2 Signal production cross sections used to normalise the $h b \mathrm{MC}$ samples. The values are taken from the NLO calculations and correspond to $\tan \beta$ of $1[96]$. 
5.3 Background MC cross sections, source of the cross section and generator level cuts where applicable.

5.4 The recorded integrated luminosity of each of the multi-jet triggers after data quality cuts for the Pass1, Pass2 and p17 analyses.

5.5 The trigger efficiencies for the various signal masses for the jet terms only. The 'Overall' efficiency is given by the integrated luminosity weighted average efficiency of the trigger lists. The uncertainties are statistical only.172

5.6 The trigger efficiencies for the various signal masses for the L3 IP $b$-tag term only. Events were required to have at least three NN tight $b$-tags. The uncertainties are statistical only.

5.7 The trigger efficiencies for various Higgs masses, when requiring at least three NN tight $b$-tagged jets. The 'Overall' efficiency is given by the integrated luminosity weighted average efficiency of the trigger lists. The uncertainties are statistical only.

5.8 Trigger efficiencies calculated using TrigSim and the analysis trigger simulation for the jet only trigger ( $\mathrm{v} 9$ - v12) measured relative to the skimming criteria $\left(3\right.$ jets $\mathrm{p}_{T}>15 \mathrm{GeV}$ and 2 jets $\left.\mathrm{p}_{T}>25 \mathrm{GeV}\right)$. The TrigSim errors are statistical only and the errors on the analysis trigger simulation includes the $9 \%$ systematic error from the 'p14' analyses (see Section 5.5.4.1).

5.9 The individual errors for the Loose SVT tagger from each source (in \%), added in quadrature to give the total error on the signal MC.

5.10 The expected and observed 95\% C.L. $\tan \beta$ limits for the MSSM at treelevel for each $m_{A}$.

5.11 The jet flavour composition predicted from Pythia MC samples of di-jet and multi-jet events, errors are statistical only.

5.12 The systematic errors on the signal in the Pass2 analysis for the Medium NN operating point. All the errors are in $\%$ and are added in quadrature to give the total error. A description of the errors can be found in Section 5.5.4.1. 
5.13 The systematic errors on the signal in the Pass2 analysis for the Tight SVT operating point. All the errors are in $\%$ and are added in quadrature to give the total error. A description of the errors can be found in Section 5.5.4.1.

5.14 The systematic errors, in $\%$, on the signal $\left(M_{h}=120 \mathrm{GeV}\right)$ for the NN and SVT taggers for each of their respective operating points. Only three operating points exist for the SVT tagger.

5.15 The systematic errors, in \%, on the background for the NN and SVT taggers for each of their respective operating points. Only three operating points exist for the SVT tagger.

5.16 The number of events in the signal, predicted background and data triple tagged distribution for the Medium NN and Tight SVT taggers. The errors on the data are statistical only, the errors on the predicted background and signal contain the statistical and systematic errors.

5.17 The expected 95\% C.L. $\tan \beta$ limits for the NN tagger and the Pass2 SVT tagger in the MSSM scheme at tree-level. No systematic errors were included in the limit setting.

5.18 The expected 95\% C.L. $\tan \beta$ limits for the Pass1 SVT tagger, Pass2 NN tagger and the Pass2 SVT tagger in the MSSM scheme at tree-level. Systematic errors were used in the limit setting.

5.19 The expected 95\% C.L. $\tan \beta$ limits for the Pass1 SVT tagger and the Pass2 SVT tagger in the MSSM scheme at tree-level for each $m_{A}$. Limits were calculated assuming the Pass1 systematic error values.

5.20 The systematic errors on the signal in the p17 analysis for the Tight NN operating point. All the errors are in $\%$ and are added in quadrature to give the total error. A description of the errors can be found in Section 5.5.4.1.

5.21 The systematic errors on the background prediction from data in the p17 analysis for the Tight NN operating point. All the errors are in \% and are added in quadrature to give the total error.

5.22 The observed and expected 95\% C.L. $\tan \beta$ exclusion limits for the p17 analysis in the MSSM scheme at tree-level. 


\section{Preface}

This thesis describes the work the author carried out between January 2003 - June 2006 as a member of the $\mathrm{D} \varnothing$ collaboration. The structure of the thesis is outlined below, followed by a description of the author's work.

Chapter 1 - A brief review of the Standard Model (SM) and the Minimal Supersymmetric extension of the Standard Model (MSSM), paying particular attention to the Higgs sector.

Chapter 2 - A description of the Tevatron and the DØ detector.

Chapter 3 - All work relevant to the Level 3 trigger, namely: improving the hit finding and tracking in the Silicon Microstrip Detector, the development and commissioning of a secondary vertexing tool and the design of two multi-jet Higgs triggers.

Chapter 4 - The development and commissioning of the Neural Network (NN) $b$-tagging tool at $\mathrm{D} \varnothing$.

Chapter 5 - The search for neutral supersymmetric Higgs bosons $(h b)$ in multi-jet events at $\mathrm{D} \varnothing$, which covers the three analyses with which I was involved.

Chapter 6 - An overview and the future outlook of the work in this thesis.

Natural units are used throughout this thesis where $c=\hbar=1$, and energy, momentum and masses are all therefore expressed in terms of electron volts $(\mathrm{eV})$.

The work the author undertook as part of this thesis is outlined below, broken into my Level 3, b-tagging and Higgs work. 
Level 3: The author has been a member of the Level 3 (L3) algorithms group since January 2003. The author's work for the L3 group centred around $b$-tagging and tracking improvements. The author alone is responsible for the L3 work documented in this chapter, except for the secondary vertex tool which was tuned with the help of a fellow PhD student.

The tracking work resulted in various improvements to the Silicon Microstrip hit finding. These upgrades were presented to and accepted by the L3 group in June 2004. The upgrades substantially improved the quality, efficiency and purity of the tracking. Several of the improvements have since been incorporated into the 'offline' reconstruction code. The improvements to the L3 tracking have resulted in more efficient L3 triggers for almost every physics group at DØ.

The L3 triggers developed for the multi-jet Higgs analysis, including the use of $b$-tagging in a trigger for the first time, were presented to and accepted by the Trigger Board. The triggers have been running online since Summer 2004, and are integral to the success of the latest multi-jet Higgs analysis. Since its demonstrated success in the multi-jet Higgs trigger, the $b$-tagging tool has been incorporated into numerous triggers.

The L3 secondary vertex $b$-tagging tool has been running online in a test trigger since Summer 2006, and will be incorporated into new physics triggers in Winter 2006. This new tool will play an important role in providing the extra rejection necessary to allow triggers to effectively function at the higher Run IIb instantaneous luminosities. As part of this work a method was developed to allow trigger development over a large sample of $b$-enriched real data events spanning a long time period, and this has since been used in the development of other tools.

b-Tagging: The author has been an active member of the $b$-ID algorithms group since November 2004, and designed and developed the first, and only, NN $b$-tagging tool at DØ. With the help of another PhD student the author 'certified' this tool for general use, and implemented the tool into the standard analysis framework. The 'certification' required the tagger to be accepted by both an editorial board and a group review. This tool has greatly increased the performance of the $b$-tagging at D $\varnothing$ and is now the official $b$-tagging tool which is used in all $b$-jet based analyses. The large improvement in the $b$-tagging efficiency has effectively doubled the sensitivity of most $b$-jet based analyses at $\mathrm{D} \varnothing$.

The author also developed a fully automated package which performs the full certification required for a $b$-tagging tool at $\mathrm{D} \varnothing$, including: determining the data $b$-tagging efficiency on $u d s g, c$ and $b$-jets, a full error evaluation and production of 
all plots needed for documentation. This package is now used for every $b$-tagging tool's certification at D $\varnothing$, and greatly simplifies a very complex procedure which needs to be repeated on a regular basis. This has long been a goal of the $b$-tagging group.

Higgs: The author has been a member of the Higgs group since October 2003. The author has participated in three generations of the ' $h b$ 'analysis. The first analysis in which the author took part was the 'p14 Pass1' analysis, which was published in Physical Review Letters, setting a new world best limit on the MSSM Higgs sector. The author carried out trigger studies as part of this analysis.

The second analysis documented is the 'p14 Pass2' analysis. The aim of the Pass2 analysis was to investigate the effect of the higher performance NN $b$-tagging in the $h b$ analysis. The author undertook all the work in this version of the analysis, and co-developed a new analysis method and background model, which was necessary due to the higher performance $b$-tagging.

The last analysis is the 'p17' analysis. The author's contribution included the design of the triggers (outlined in Chapter 3) which collected two thirds of the data, the joint development of the new background model and analysis method, and the Neural Network $b$-tagging. This version of the analysis has set a new preliminary world best limit on the MSSM Higgs sector, as presented at ICHEP 2006, and will be published early 2007 . 


\section{Chapter 1}

\section{Theory}

The Standard Model (SM) of particle physics is a very successful model which describes to a high degree of accuracy the interactions of the fundamental particles. The SM is not however a complete theory and the origin of mass in the SM has not yet been resolved. The most popular theory to introduce mass into the SM is the Higgs mechanism. Searches for the Higgs boson are thus currently one of the highest priorities in high energy physics.

Supersymmetry (SUSY) is a popular extension of the SM which creates a symmetry between fermions and bosons. SUSY naturally explains some problems within the SM, and allows for the unification of all the forces and the inclusion of gravity into the SM. At present no evidence for SUSY has been found, although neither has it been excluded.

A brief overview of the SM is given in Section 1.1. The Higgs mechanism is explained in Section 1.1.1, and constraints on the mass of the SM Higgs are outlined in Section 1.1.2. The motivation for SUSY, along with an introduction to the minimal supersymmetric extension to the SM (MSSM), is covered in Section 1.2. The Higgs sector in the MSSM is covered in Section 1.2.1, along with the recent search results for the MSSM Higgs bosons.

This chapter is a brief introduction to the SM and SUSY, and more complete reviews can be found in $[1,2]$ and $[3,4,5]$ respectively.

\subsection{The Standard Model}

This section provides a brief overview of how the forces, via their mediating bosons, are 'naturally' introduced into the SM by requiring systems to be invariant under certain symmetry transformations. Group transformations which vary in space and 
time are called 'local gauge transformations'. By forcing the Lagrangian ${ }^{1}$ of a free particle to be invariant under a local group transformation, it is necessary to introduce a 'gauge field' to maintain the invariance of the Lagrangian. These gauge fields are actually the force mediating spin-1 bosons. The use of local gauge invariance is best demonstrated using a free Dirac field, $\Psi=\Psi(x)$ and $\bar{\Psi}=\Psi^{\dagger} \gamma^{0}$, whose Lagrangian is given by:

$$
\mathcal{L}_{\text {Dirac }}=\bar{\Psi}\left(i \gamma^{\mu} \partial_{\mu}-m\right) \Psi
$$

where $\gamma^{\mu}$ are the $4 \times 4$ gamma matrices. A U(1) local gauge transformation of the Dirac fields is defined as:

$$
\begin{gathered}
\Psi \rightarrow \Psi^{\prime}=e^{i \theta(x)} \Psi \\
\bar{\Psi} \rightarrow \bar{\Psi}^{\prime}=e^{-i \theta(x)} \bar{\Psi}
\end{gathered}
$$

where $\theta(x)$ is a function of the space-time co-ordinates. Substituting Equation 1.2 into Equation 1.1 proves that the Lagrangian is clearly not invariant in its current form:

$$
\mathcal{L}_{\text {Dirac }} \rightarrow \mathcal{L}_{\text {Dirac }}^{\prime}=\mathcal{L}_{\text {Dirac }}-\bar{\Psi} \gamma^{\mu} \partial_{\mu} \theta(x) \Psi
$$

To maintain the invariance of the Lagrangian a real gauge field, $A_{\mu}$, is introduced whose transformation exactly cancels out the extra term in Equation 1.3:

$$
A_{\mu} \rightarrow A_{\mu}^{\prime}=A_{\mu}-\frac{1}{e} \partial_{\mu} \theta(x) .
$$

For completeness an invariant kinetic term for the gauge field is also added to the Lagrangian:

$$
\mathcal{L}^{K . E .}=-\frac{1}{4} F_{\mu \nu} F^{\mu \nu}
$$

where $F_{\mu \nu}=\partial_{\mu} A_{\nu}-\partial_{\nu} A_{\mu}$. Replacing the derivative with the covariant derivative, $D_{\mu}$, defined as:

$$
D_{\mu} \equiv \partial_{\mu}+i e A_{\mu}
$$

ensures that the extra term is cancelled out and the Lagrangian is invariant under the U(1) local gauge transformation. The resulting Lagrangian now describes

\footnotetext{
${ }^{1}$ Technically it is the Lagrangian density, and the actually Lagrangian is given by $L=\int \mathcal{L} d^{3} x$.
} 
the interactions of electrons and photons - quantum electrodynamics (QED). By requiring the invariance of a free Dirac field under a $U(1)$ local gauge transformation, the free system has been changed into an interacting one. The final QED Lagrangian is given by:

$$
\begin{aligned}
\mathcal{L}_{Q E D} & =\bar{\Psi}\left(i \gamma^{\mu} D_{\mu}-m\right) \Psi-\frac{1}{4} F_{\mu \nu} F^{\mu \nu} \\
& =\bar{\Psi}\left(i \gamma^{\mu} \partial_{\mu}-m\right) \Psi-\underbrace{e \bar{\Psi} \gamma^{\mu} \Psi A_{\mu}}_{\text {Interaction Term }}-\frac{1}{4} F_{\mu \nu} F^{\mu \nu} .
\end{aligned}
$$

A mass term for $A_{\mu}$ is not included, as a term such as $m A_{\mu} A^{\mu}$ is not invariant. In the SM the gauge bosons of the weak and strong forces are introduced in an analogous way, but using different group structures to represent the different symmetries. For instance, the strong force is introduced into the SM Lagrangian by requiring invariance under $\mathrm{SU}(3)_{C}$ local gauge transformations. The 8 generators ${ }^{2}$ of the $\mathrm{SU}(3)$ group correspond to the 8 gluons.

The unified EM and weak forces are introduced by requiring invariance under $\mathrm{SU}(2)_{L} \otimes \mathrm{U}(1)_{Y}$ local gauge transformations $[11,12]$. The $\mathrm{SU}(2)_{L}$ transformations are in weak isospin $(\mathbf{T})$ space and the $\mathrm{U}(1)_{Y}$ transformations are in hypercharge $(\mathrm{Y})$ space. The three generators of the $\mathrm{SU}(2)_{L}$ group correspond to the $\mathrm{W}_{\mu}^{1}, \mathrm{~W}_{\mu}^{2}$ and $\mathrm{W}_{\mu}^{3}$ gauge bosons with a coupling $g$, and the single generator of the $\mathrm{U}(1)_{Y}$ group to the $\mathrm{B}_{\mu}$ boson with a coupling $g^{\prime}$. The EM charge, $\mathrm{Q}$, is given by $Q=T^{3}+Y / 2$ where $T^{3}$ is the eigenvalue of the third component of isospin, and the couplings are related by $g^{\prime}=g \tan \theta_{W}$, where $\theta_{W}$ is the weak mixing angle. The physical bosons (photon, $\mathrm{W}^{ \pm}$and $\mathrm{Z}^{0}$ ) exist as linear superpositions of the gauge fields, and are given by:

$$
\begin{aligned}
W^{ \pm} & \equiv\left(W_{\mu}^{1} \mp i W_{\mu}^{2}\right) \sqrt{2} \\
Z_{\mu} & \equiv \cos \theta_{W} W_{\mu}^{3}-\sin \theta_{W} B_{\mu} \\
A_{\mu} & \equiv \cos \theta_{W} B_{\mu}-\sin \theta_{W} W_{\mu}^{3} .
\end{aligned}
$$

The gauge bosons are all massless to maintain the invariance of the Lagrangians, however this is at odds with the experimental evidence of massive $\mathrm{Z}^{0}$ and $\mathrm{W}^{ \pm}$bosons. Clearly a mechanism is needed which introduces mass terms into the SM in an invariant way; one such theory is the Higgs mechanism.

\footnotetext{
${ }^{2} \mathrm{SU}(\mathrm{n})$ groups have $n^{2}-1$ generators.
} 


\subsubsection{The Higgs Mechanism}

The section describes the Higgs mechanism [6, 7, 8]. An illustrative example is given below of how the Higgs mechanism can give mass to a gauge boson, and the implementation of the Higgs mechanism into the SM is covered at the end of this section.

The Higgs mechanism is best illustrated using a U(1) locally gauge invariant Lagrangian for a complex scalar field, $\phi$ (analogous to the Lagrangian derived in Section 1.1):

$$
\begin{aligned}
\phi & =\frac{1}{\sqrt{2}}\left(\phi_{1}+i \phi_{2}\right) \\
\phi^{*} & =\frac{1}{\sqrt{2}}\left(\phi_{1}-i \phi_{2}\right) .
\end{aligned}
$$

The U(1) gauge invariant Lagrangian for this field is (see Section 1.1):

$$
\mathcal{L}=\underbrace{\left(\partial^{\mu}+i q A^{\mu}\right) \phi^{*}\left(\partial_{\mu}-i q A_{\mu}\right) \phi-\frac{1}{4} F^{\mu \nu} F_{\mu \nu}}_{\text {Kinetic Term }}-\underbrace{V(\phi)}_{\text {Potential Term }}
$$

The first two terms are the kinetic terms, and the third term, $V(\phi)$, is the potential term which is defined as:

$$
V(\phi)=\mu^{2} \phi^{*} \phi+\lambda\left(\phi^{*} \phi\right)^{2}
$$

The potential for $\mathrm{V}(\phi)$ is shown in Fig. 1.1 for the case of $\mu^{2}>0$ and $\mu^{2}<0$. In the case of $\mu^{2}>0$ the minimum of the potential is at $\phi=0$ and the Lagrangian describes a scalar field with mass $\sqrt{2} \mu$. The more interesting case is that for $\mu^{2}<0$, where there is an unstable maximum at $\phi=0$ and a minimum mapped out by a circle:

$$
\phi_{1, \text { min }}^{2}+\phi_{2, \text { min }}^{2}=v^{2} \text { with } v=\sqrt{\frac{-\mu^{2}}{\lambda}},
$$

where $v$ is the vacuum expectation value. The perturbative expansion of the Lagrangian has to be performed around the classical minimum, and by choosing a minimum we are breaking the symmetry of the theory. This is 'spontaneous symmetry breaking', by choosing a particular minimum the symmetry of the theory has been broken. The minimum is chosen as: 

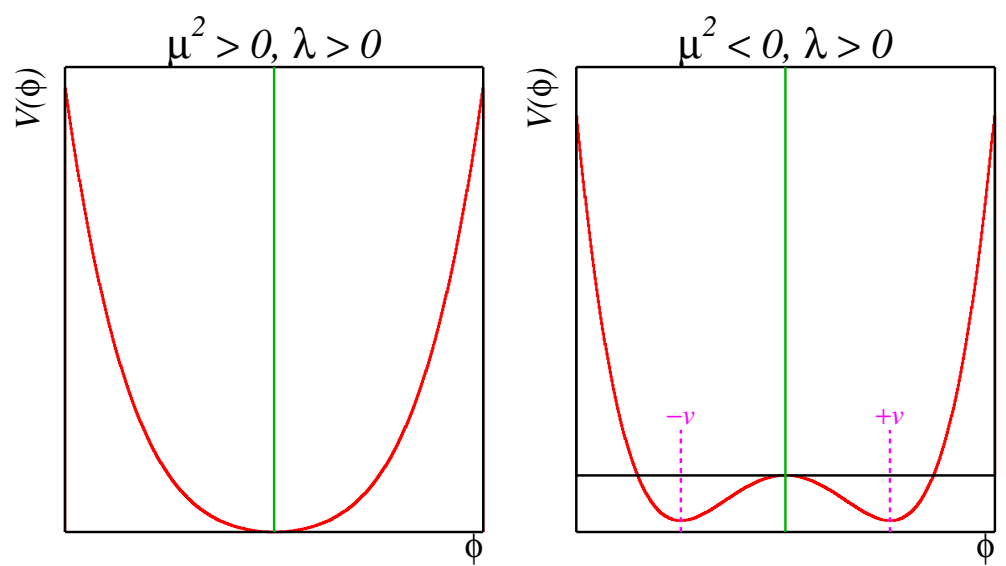

Figure 1.1: Graphical representation of $V(\phi)$ [9].

$$
\begin{aligned}
\phi_{1} & =v \\
\phi_{2} & =0
\end{aligned}
$$

and two new fields can be defined at the minimum:

$$
\begin{array}{r}
\eta=v-\phi_{1} \\
\zeta=\phi_{2} .
\end{array}
$$

Substituting Equation 1.18 into Equation 1.13 gives:

$$
\begin{aligned}
\mathcal{L} & =\underbrace{\frac{1}{2}\left(\partial_{\mu} \eta\right)^{2}-v^{2} \lambda \eta^{2}}_{\text {Scalar Particle }}+\underbrace{\frac{1}{2}\left(\partial_{\mu} \zeta\right)^{2}}_{\text {Goldstone Boson }}-\underbrace{\frac{1}{4} F^{\mu \nu} F_{\mu \nu}}_{\text {K.E. Term }} \\
& +\underbrace{\frac{1}{2} e^{2} v^{2} A_{\mu} A^{\mu}}_{\text {Mass Term }}-\underbrace{e v A_{\mu} \partial^{\mu} \zeta}_{\text {Interaction Term }}+\ldots
\end{aligned}
$$

The first two terms describe a scalar particle $(\eta)$ of mass $\sqrt{2 v^{2} \lambda}$, and the third term describes a 'Goldstone' boson, $\zeta$, a massless scalar spin-0 particle which always results from the breaking of a continuous global symmetry [10]. The fourth term provides a mass term for the previously massless boson field $A^{\mu}$ as desired.

The above Lagrangian does not represent the fundamental particles in the theory. The field $\zeta$ has a bilinear interaction term with $A^{\mu}$ and it is also a massless boson. By picking the 'unitary gauge', defined as: 


$$
\theta=-\tan ^{-1}\left(\phi_{2} / \phi_{1}\right)
$$

the $\zeta$ field can be transformed away. Replacing $\eta \rightarrow h$, the Lagrangian becomes:

$$
\begin{aligned}
\mathcal{L}_{\text {scalar }}^{\prime \prime} & =\frac{1}{2}\left(\partial_{\mu} h\right)^{2}-\frac{1}{4} F^{\mu \nu} F_{\mu \nu}-v^{2} \lambda h^{2}+\frac{1}{2} e^{2} v^{2} A_{\mu} A^{\mu} \\
& -\lambda v h^{3}-\frac{1}{4} \lambda h^{4}+\frac{1}{2} e^{2} A_{\mu}^{2} h^{2}+v e^{2} A_{\mu}^{2} h .
\end{aligned}
$$

There are two real fields left, a massive scalar field $h$ (the Higgs particle) and a massive gauge field $A_{\mu}$. From the initial massive complex scalar field introduced into the Lagrangian, the $A_{\mu}$ field has 'absorbed' an extra degree of freedom, leaving a massive scalar Higgs field.

\subsubsection{The Higgs Mechanism in the Standard Model}

The above example is $\mathrm{U}(1)$, in the SM the Higgs mechanism couples to $\mathrm{SU}(2)$, thus we introduce a doublet of complex scalar fields which has four degrees of freedom. The Higgs field spontaneously breaks the symmetry of the SM Lagrangian and in the process imparts three of its degrees of freedom to the weak gauge bosons, with the remaining degree of freedom appearing as the massive scalar Higgs field. The vacuum remains invariant under EM charge $(\mathrm{Q})$ hence electric charge is conserved and the photon remains massless.

The mass terms for the fermions, f, can be generated using the same Higgs doublet in a gauge invariant way. The Yukawa couplings are of the form:

$$
\mathcal{L}_{Y u k a w a}=-G_{f}\left(\bar{\Psi}_{L} \Phi \Psi_{R}+\bar{\Psi}_{R} \Phi^{\dagger} \Psi_{L}\right)
$$

where $G_{f}$ are the Yukawa coupling constants, determined from experiment. The mass of the fermions and their interaction with the Higgs can be generated by substituting the broken Higgs field transformed into the unitary gauge into Equation 1.21 , as shown below for the case of an electron:

$$
\mathcal{L}_{Y u k a w a}=-m_{e}\left(\bar{e}_{L} e_{R}+\bar{e}_{R} e_{L}\right)-\frac{m_{e}}{v}\left(\bar{e}_{L} e_{R}+\bar{e}_{R} e_{L}\right) h .
$$

The first term is the mass of the electron, with $m_{e}=G_{e} v / \sqrt{2}$, and the second term is its interaction with the Higgs. The coupling is proportional to the mass of the electron. A similar procedure can be followed to impart mass to the quarks, whose coupling to the Higgs is again proportional to their mass. 


\subsubsection{Constraints on the Higgs}

Although the Higgs mechanism is successful in generating the necessary mass terms in the SM, the Higgs boson has not been observed. The constraints from direct searches, indirect measurements and theory on the Higgs mass are outlined in the following sections.

\subsubsection{Direct Searches}

The most stringent limit on the mass of the Higgs particle has been set by direct searches carried out at LEP. The LEP experiments performed a direct search for the Higgs boson using $2461 \mathrm{pb}^{-1}$ of data collected at centre of mass energies from 189 - $209 \mathrm{GeV}$. The primary production process was $e^{+} e^{-} \rightarrow H Z$; all Z decays were included for the $H$ to $b \bar{b}$ channel, but only $\mathrm{Z}$ decays to quarks were included for the $H$ to $\tau^{+} \tau^{-}$channel. A lower limit was set on the Higgs boson mass of $114.4 \mathrm{GeV}$ at $95 \%$ confidence level (CL) [13].

Direct searches have also been carried out at both D $\varnothing$ and CDF. Upper limits have been set on the production cross section of a SM Higgs in associated production $\left(Z H \rightarrow \nu \nu b \bar{b}, W H \rightarrow e \nu b \bar{b}, W H \rightarrow \mu \nu b \bar{b}\right.$ and,$\left.W H \rightarrow W W^{+} W^{-}\right)$and gluon fusion $(H \rightarrow W W)$. The current combined limit from both experiments for all search channels is presented in Fig. 1.2 [14]. The expected limit indicates the limit setting potential of the experiment, and is derived from the background and signal distributions assuming no signal production. The observed limit is the actual limit on signal production derived when also taking into account the experimental data. The observed limit at the Tevatron is currently a factor of 10(4) from the SM cross section at a mass of 115 (160) GeV, although with the expected increases in luminosity and improved analysis techniques (see for example the $b$-tagging improvements in Chapter 4) this factor will decrease rapidly. Previous studies have shown that $\sim 2 \mathrm{fb}^{-1}$ is needed for sensitivity to a SM Higgs of mass $\sim 115 \mathrm{GeV}[15]$.

\subsubsection{Indirect Experimental Measurements}

Most electroweak (EW) parameters are sensitive to the Higgs via higher order loop corrections and it is thus possible to use these corrections to place indirect constraints on the mass of the Higgs. Precision measurements of $18 \mathrm{EW}$ parameters, such as the mass and width of the $\mathrm{W}$ boson, have been combined in a global fit with the Higgs mass, using high- $Q^{2}$ data from LEP, SLC and the Tevatron. The $\Delta \chi^{2}\left(m_{H}\right)=$ $\chi_{\min }^{2}\left(m_{H}\right)-\chi_{\min }^{2}$ of the fit as a function of Higgs mass is shown in Fig. 1.3. The minimum of the $\Delta \chi^{2}$ curve corresponds to $85_{-28}^{+39} \mathrm{GeV}$ at $68 \%$ CL and an upper limit 


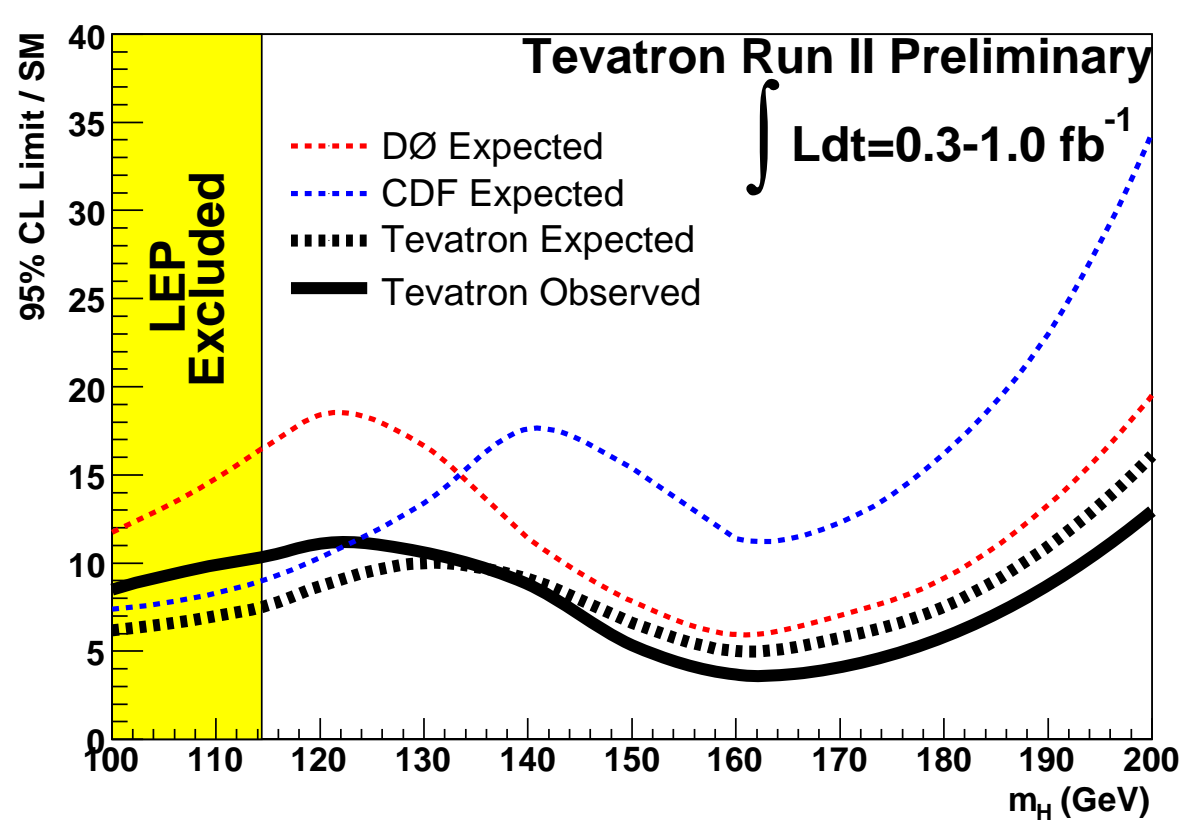

Figure 1.2: The ratio of the expected and observed 95\% CL limits to the SM cross section for the combined CDF and DØ analyses.

is set on the Higgs mass of $m_{H}<199 \mathrm{GeV}$ at 95\% CL [16]. The largest sources of error in the fit are due to the mass of the W-boson and the mass of the $t$-quark.

\subsubsection{Theoretical Constraints}

Constraints on the mass of the Higgs can be derived by requiring that $\lambda$, the constant of the quartic term in the Higgs potential (see Equation 1.14), remains positive and finite up to an energy scale $\Lambda$, where new physics appears. Figure 1.4 shows the theoretical limits on the mass of the Higgs as a function of $\Lambda$ [17]. The theoretical limits allow a Higgs boson up to the Planck scale ${ }^{3}$, in which case the Higgs mass is restricted to be between 130 and $190 \mathrm{GeV}$. If $\Lambda$ is closer to $1 \mathrm{TeV}$, then the Higgs mass is constrained to be in the range $85<m_{h}<420 \mathrm{GeV}$. Additionally, unitarity requirements in the $W_{L}$ scattering process place an upper limit of $1 \mathrm{TeV}$ on the Higgs mass, this requirement applies to all SM extensions which involve a Higgs $[18]$.

\subsection{Limitations of the Standard Model and Su- persymmetry}

Though the SM is a very successful theory, it is not complete. For example:

\footnotetext{
${ }^{3}$ The Planck scale is the energy at which quantum gravity becomes important.
} 


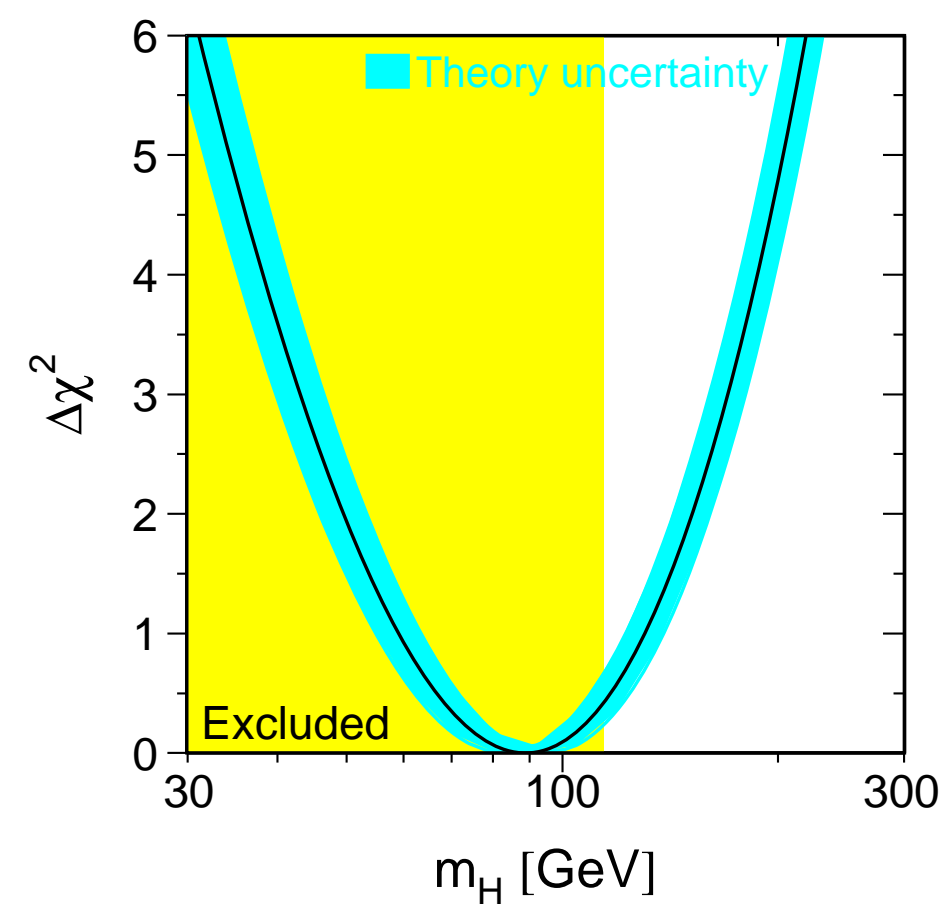

Figure 1.3: The $\Delta \chi^{2}\left(m_{H}\right)=\chi_{\min }^{2}\left(m_{H}\right)-\chi_{\min }^{2}$ of the fit to the EW parameters and the Higgs mass as a function of the Higgs mass [16]. The band represents the theoretical uncertainty, and the vertical band shows the $95 \%$ confidence level exclusion limit found from the direct searches at LEP.

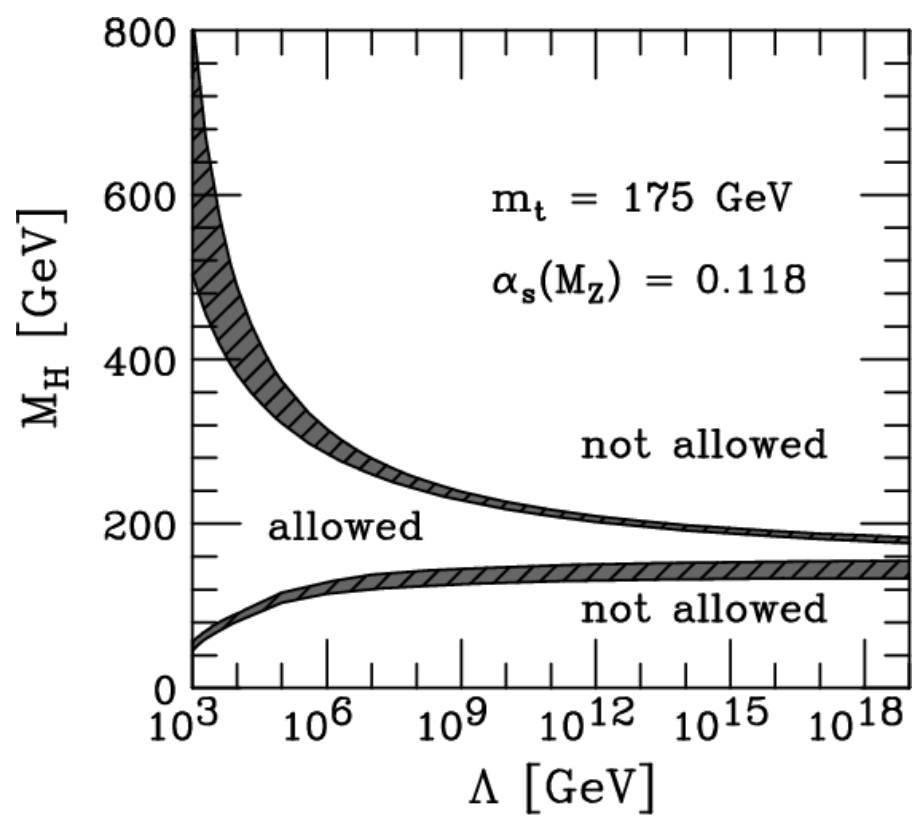

Figure 1.4: The theoretical limits on the mass of the Higgs $\left(M_{H}\right)$ as a function of $\Lambda$, the energy where new physics appears [17]. 
- The Higgs particle is expected to have a mass of the order of the EW scale (several hundred $\mathrm{GeV}$ ), although loop corrections push the mass to values of the order of the highest energy scale at which the SM is valid. This could be of the order of the Planck scale i.e. $10^{19} \mathrm{GeV}$. Correcting the Higgs mass to be of the correct order by fine tuning the parameters is possible, although this is considered to be an unsatisfactory approach, and the fine tuning has to be retuned for every order of perturbation theory. The large difference between the 'natural' Higgs mass and the EW scale is known as the 'hierarchy' problem, and the constant retuning required to maintain a Higgs mass on the order of the EW energy scale the 'fine tuning' problem [3].

- In Grand Unified Theories (GUT) the coupling constants for the strong weak and EM forces are expected to converge at high energy. In the SM the coupling constants never all converge at a single value [4].

Supersymmetry addresses both of these issues by creating a new symmetry between the fermions and the bosons. A supersymmetric partner, or 'sparticle', is introduced for every particle. The sparticles have the same quantum numbers as their SM partner, except for the spin which is altered from half integer to full integer or vice-versa. The MSSM is the simplest of the supersymmetric extensions of the SM, but still results in the inclusion of 105 extra parameters [5]. Since no supersymmetric partners have ever been seen, it is assumed that the supersymmetric particles have a higher mass than the particles.

Supersymmetry is a popular theory because:

- It solves the hierarchy problem as the extra loops from the sparticles cancel out the divergent loop terms in the SM.

- The three coupling constants converge in SUSY due to corrections caused by the sparticles, as shown in Fig. 1.5.

- The mathematical framework of SUSY allows spin-2 particles to be introduced. Gravity is postulated to be mediated by a spin-2 particle, and this could therefore allow for the future inclusion of gravity into the theory. 

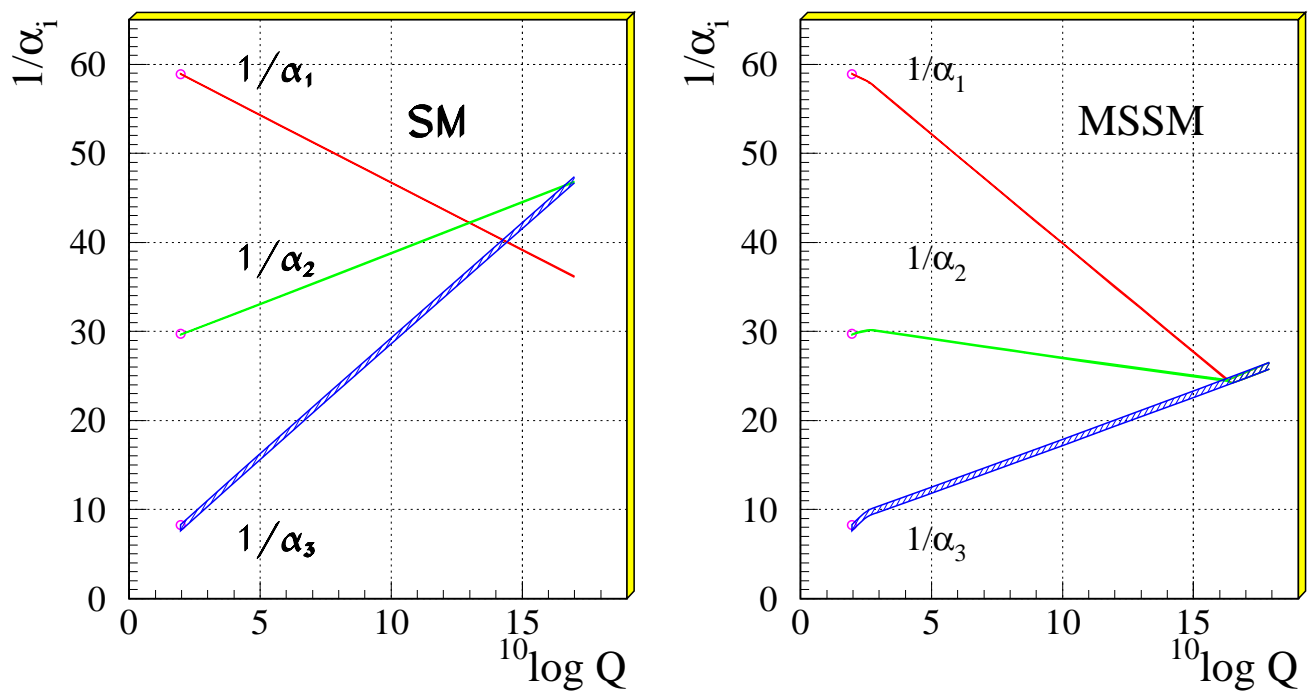

Figure 1.5: The running values of the inverse of the coupling constants as a function of the energy scale, Q, in the SM (left) and the MSSM (right) [4], where $\alpha_{1}, \alpha_{2}$ and $\alpha_{3}$ are the U(1), SU(2) and $\mathrm{SU}(3)$ coupling constants respectively.

\subsubsection{Higgs Sector of the MSSM}

In the MSSM Higgs sector there are two Higgs doublets: one which couples only to down-type fermions and the other which couples only to up-type fermions. The vacuum expectation values of these two fields are referred to as $v_{u}$ and $v_{d}$ respectively, and their ratio is defined as:

$$
\tan \beta=v_{u} / v_{d} .
$$

The Higgs doublets have 8 degrees of freedom, three of which are 'absorbed' by the EW gauge bosons. This leaves five 5 physical Higgs bosons: three neutral (one $\mathrm{CP}$-odd, $\mathrm{A}$, and two $\mathrm{CP}$ even, $\mathrm{h}$ and $\mathrm{H})$ and two charged $\left(H^{ \pm}\right)$. All couplings to fermions and the masses of the Higgs particles can be deduced once two parameters from the Higgs sector are defined. These are chosen here as $m_{A}$ and $\tan \beta$ [3].

At tree level the masses of the CP-even Higgs bosons are related to the mass of the CP-odd Higgs and the mass of the Z-boson $\left(m_{Z}\right)$ by :

$$
m_{h, H}^{2}=\frac{1}{2}\left(m_{A}^{2}+m_{Z}^{2} \mp \sqrt{\left(m_{A}^{2}+m_{Z}^{2}\right)^{2}-4 m_{A}^{2} m_{z}^{2} \cos ^{2} 2 \beta}\right) .
$$

The masses of the CP-even Higgs bosons are further constrained by the following relationships:

$$
\begin{aligned}
m_{h} & \leq m_{Z} \mid \cos (2 \beta \mid) \\
m_{H} & \geq \sqrt{m_{A}^{2}+m_{Z}^{2} \sin ^{2} 2 \beta} .
\end{aligned}
$$


The masses of the charged Higgs bosons are given by:

$$
m_{H^{ \pm}}^{2}=m_{A}^{2}+m_{W}^{2} .
$$

At tree level, the mass of $h$ appears to be less than or equal to the mass of the $Z$, however due to radiative corrections the mass actually has an upper limit of $\sim 130 \mathrm{GeV}[3]$. The SM coupling of the CP-odd Higgs boson to the bottomquark is enhanced by a factor of $\tan \beta$, and therefore the production cross section is enhanced by $\tan ^{2} \beta$. The coupling of the CP-even states, $h$ and $H$, are enhanced by $-\sin \alpha / \cos \beta$ and $\cos \alpha / \cos \beta$ respectively, where $\alpha$ is the mixing angle between $h$ and $H$, and is given by:

$$
\cos ^{2}(\beta-\alpha)=\frac{m_{h}^{2}\left(m_{Z}^{2}-m_{h}^{2}\right)}{m_{A}^{2}\left(m_{H}^{2}-m_{h}^{2}\right)} .
$$

The joint production cross section of the $h$ and $H$ Higgs bosons is equal to the production cross section of $A$ at high $\tan \beta$ (see Section 5.3.1.1 for more details). At large $\tan \beta$ this results in an enhancement factor of $\sim 2 \tan ^{2} \beta$ for the total production cross section of $\mathrm{A}, \mathrm{h}$ and $\mathrm{H}$ bosons in association with $b$-quarks with respect to the SM production cross section [19].

Large $\tan \beta$ values are favoured by some models, as it naturally explains the difference in mass between the bottom and top quarks [20].

\subsubsection{MSSM Higgs Searches}

Combined limits from the four LEP experiments have excluded at 95\% CL MSSM neutral Higgs bosons below $\mathrm{m}_{h}<92.9 \mathrm{GeV}$ and $\mathrm{m}_{A}<93.4 \mathrm{GeV}$ for all $\tan \beta$, and have excluded $\tan \beta$ values between 0.7 and 2.0 for all $\mathrm{m}_{A / h}$ values [21].

Searches at the Tevatron have further constrained the MSSM Higgs bosons firstly at $\mathrm{CDF}[22]$ and then at $\mathrm{D} \varnothing[23,24,25,26]$. The current preliminary world best limit has been set in the latest $\mathrm{D} \varnothing$ analysis [26] which has set limits on $\tan \beta$ from 50 - 100 for $\mathrm{m}_{A}$ ranging from $100-170 \mathrm{GeV}$. This result [26], along with its predecessor [24] are documented in Chapter 5. 


\section{Chapter 2}

\section{The Tevatron and the DØ Detector}

\subsection{The Tevatron}

The Fermi National Accelerator Laboratory (Fermilab) [27], as shown in Fig. 2.1, consists of a series of accelerators which produce protons and antiprotons, and accelerate them up to $150 \mathrm{GeV}$ for injection into the Tevatron. The Tevatron is currently the highest energy operational particle accelerator in the world. The accelerator is $6.3 \mathrm{~km}$ in circumference and collides beams of protons and antiprotons at a centre of mass energy of $1.96 \mathrm{TeV}$. Both beams are circulated in the same beam pipe but in opposite directions, and are brought to focus at two points: the D $\varnothing$ and CDF detectors. Run I of the Tevatron took place between 1992-1996, and Run II began in 2001. Run II is split into two sections, Run IIa and Run IIb. Run IIa finished in April 2006, and all data used in this thesis were collected during this run. Both detectors were upgraded to operate at the higher instantaneous luminosities expected in Run IIb, which began in June 2006. Run II is currently planned to last until the end of 2009, with a final total integrated luminosity of up to $8 \mathrm{fb}^{-1}$. The operating parameters for the Tevatron in Run I and II are shown in Table 2.1. Figure 2.2 shows the peak instantaneous luminosity achieved during Run IIa; as can be seen the goals were exceeded. Instantaneous luminosities in Run IIb are expected to reach $\sim 3 \times 10^{32} \mathrm{~cm}^{-2} \mathrm{~s}^{-1}$.

\subsection{The DØ Detector}

The D $\varnothing$ detector, shown in Fig. 2.3, has a symmetrical design of concentric cylindrical sub-detectors centred around the collision point. The innermost layer is the 


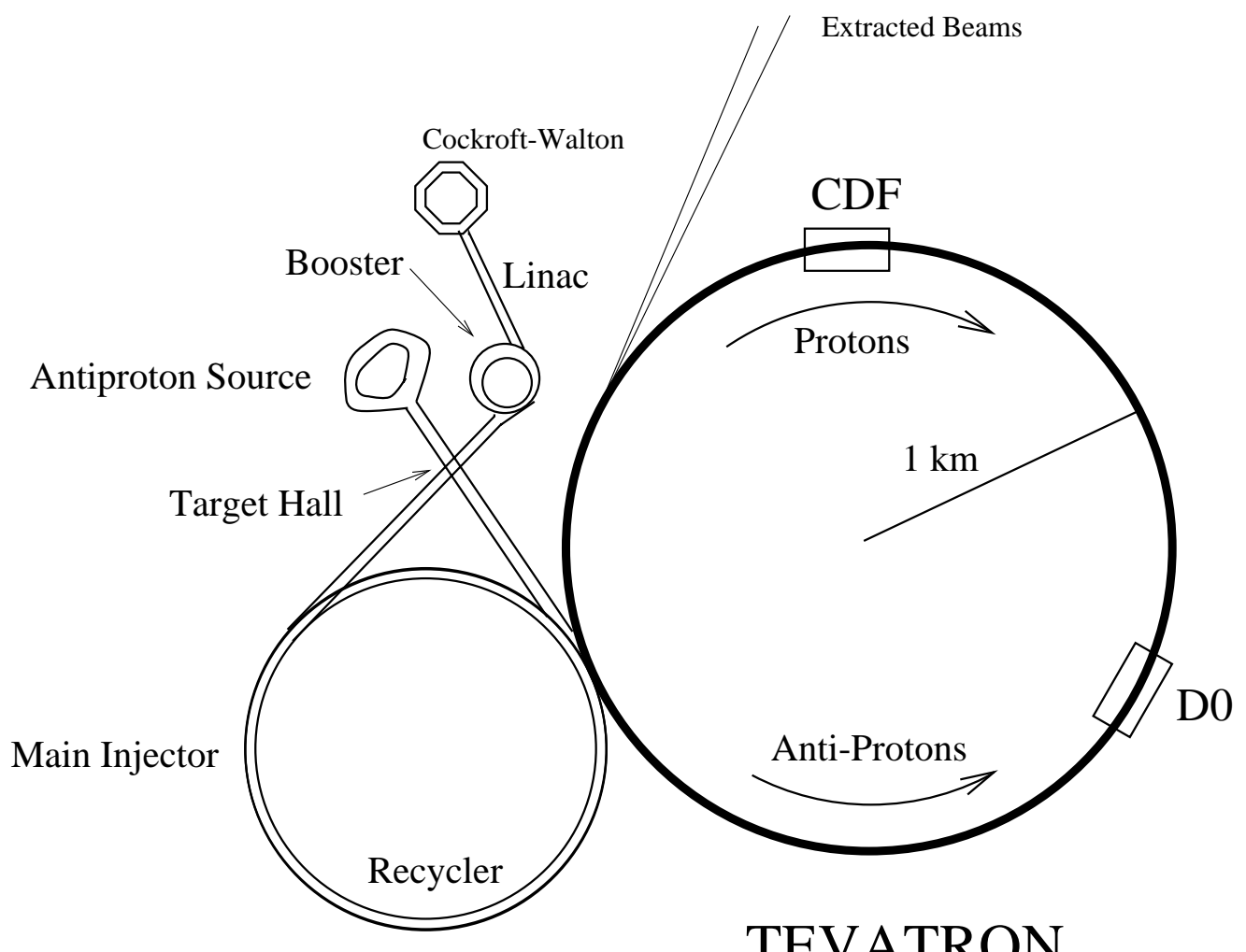

Figure 2.1: The system of accelerators at the Fermi National Accelerator Laboratory used to produce and accelerate protons and antiprotons.

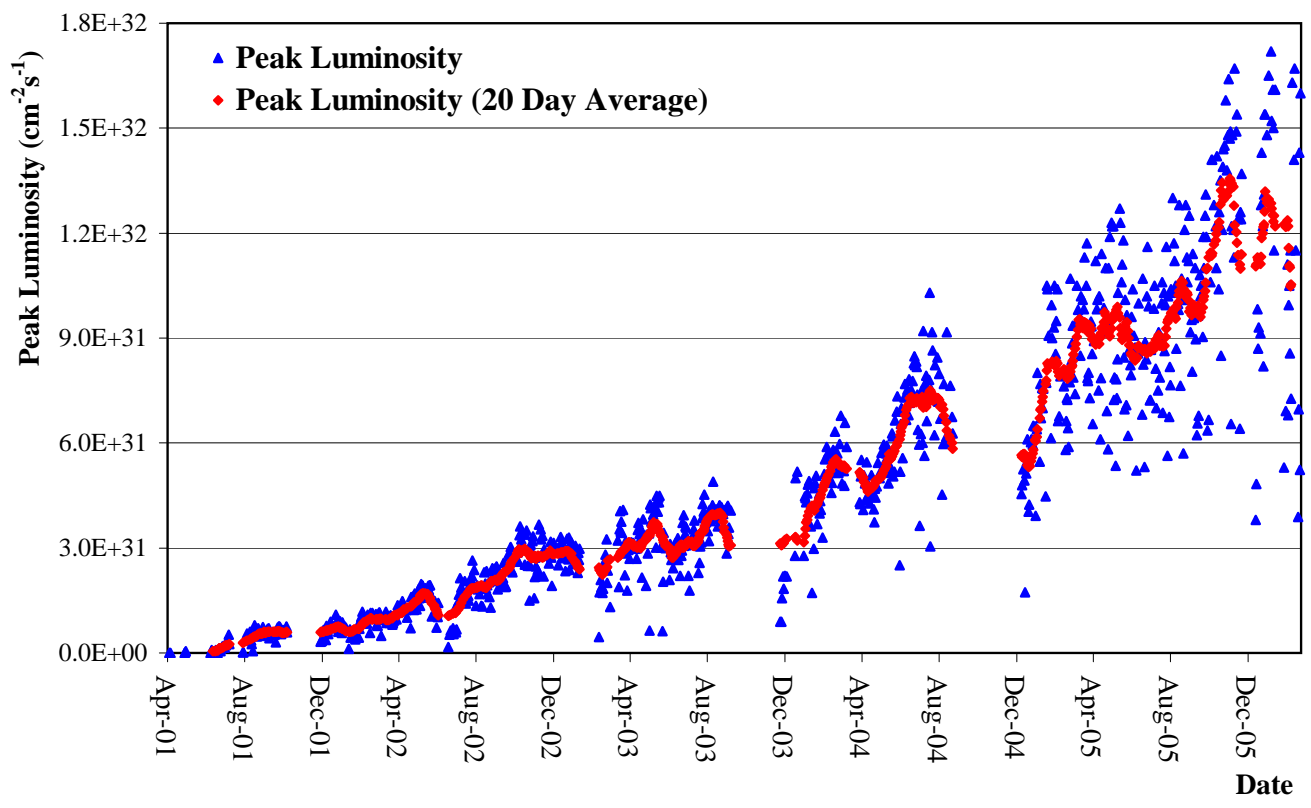

Figure 2.2: The peak instantaneous luminosity delivered by the Tevatron during Run IIa [28]. 


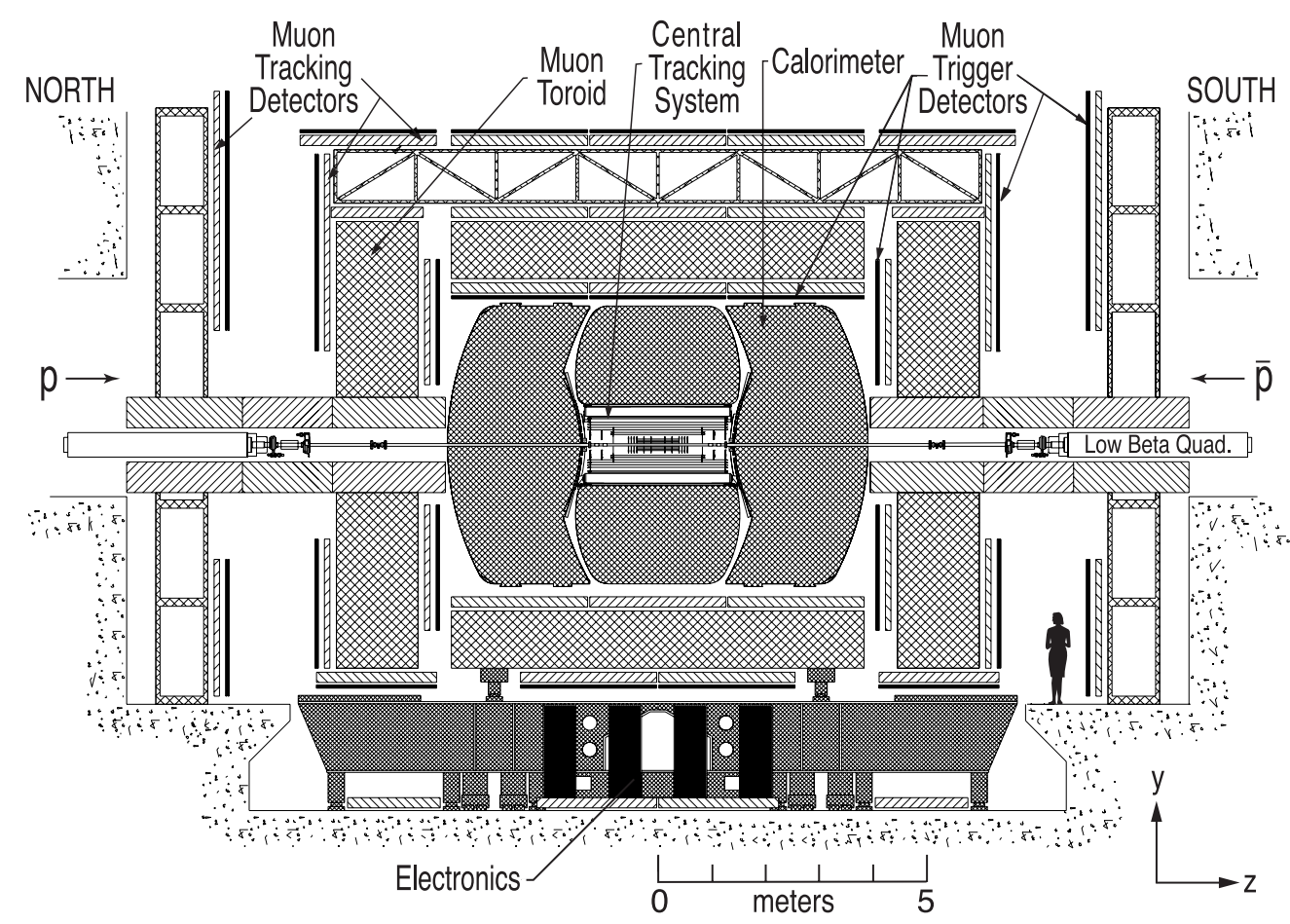

Figure 2.3: A cross section of the $D \emptyset$ detector. The central tracking region is shown in more detail in Fig. 2.4.

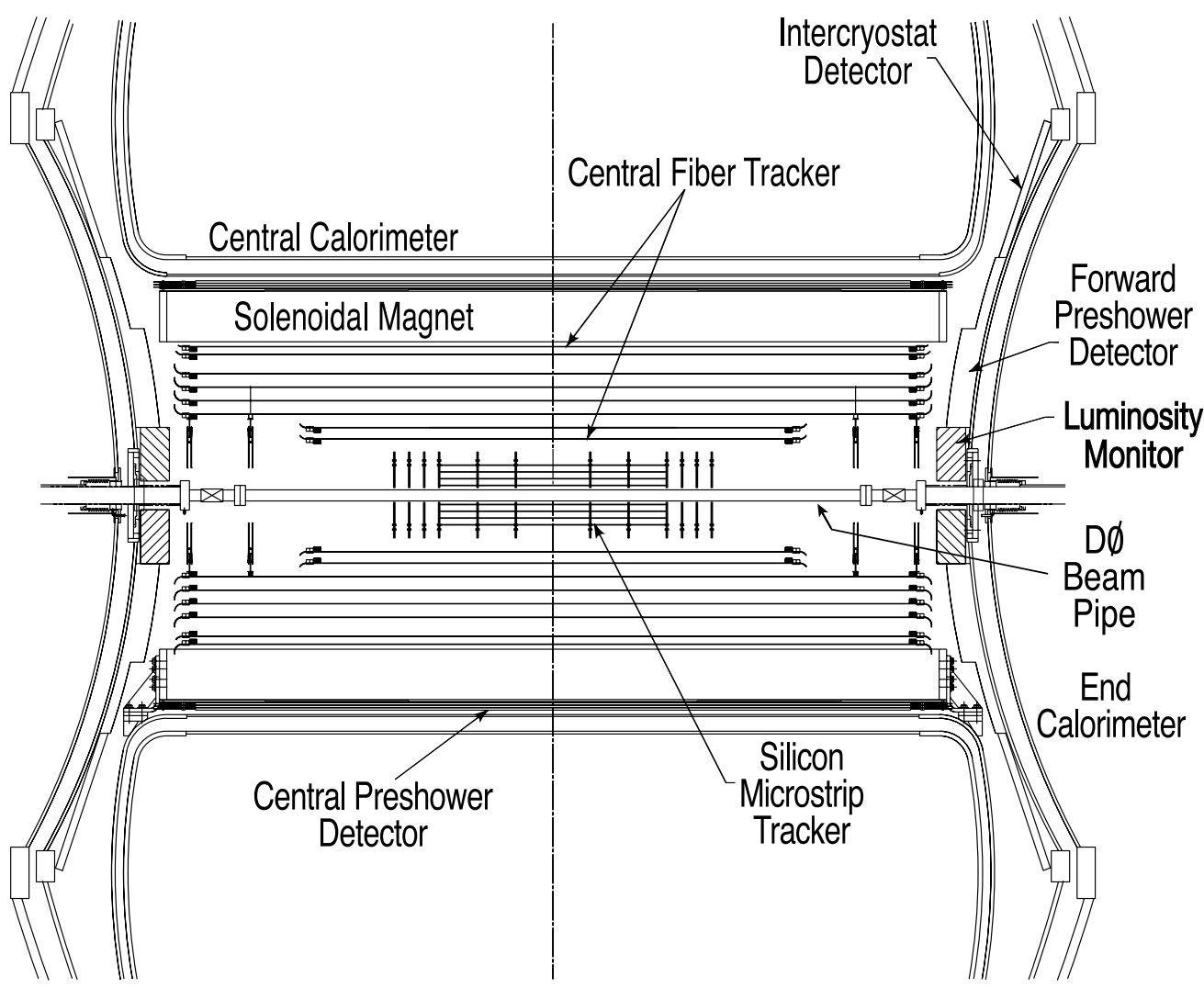

Figure 2.4: The central tracking region in the DØ detector. 


\begin{tabular}{|c|c|c|}
\hline & Run I & Run II \\
\hline Energy $p \bar{p}(\mathrm{GeV})$ & 900 & 980 \\
\hline Proton bunches & 6 & 36 \\
\hline Protons/bunch & $2.3 \times 10^{11}$ & $2.7 \times 10^{11}$ \\
\hline Antiproton bunches & 6 & 36 \\
\hline Antiprotons/bunch & $5.5 \times 10^{10}$ & $3.0 \times 10^{10}$ \\
\hline Bunch spacing (ns) & 3500 & 396 \\
\hline Peak luminosity $\left(\mathrm{cm}^{-2} \mathrm{~s}^{-1}\right)$ & $0.16 \times 10^{32}$ & $\sim 1 \times 10^{32}$ \\
\hline Luminosity ( $\mathrm{pb}^{-1} /$ week$)$ & 3.2 & 17.3 \\
\hline Interactions per crossing & 2.5 & 2.3 \\
\hline
\end{tabular}

Table 2.1: The Tevatron operating parameters in Run I and II [30].

central tracking system, shown in Fig. 2.4, which is followed by the calorimeter and then the muon detectors. All sub-detectors are described in more detail in the following sections, and full details can be found in [29]. The D $\varnothing$ detector functions with an average data-taking efficiency ${ }^{1}$ of between $85-90 \%$ as shown in Fig. 2.5.

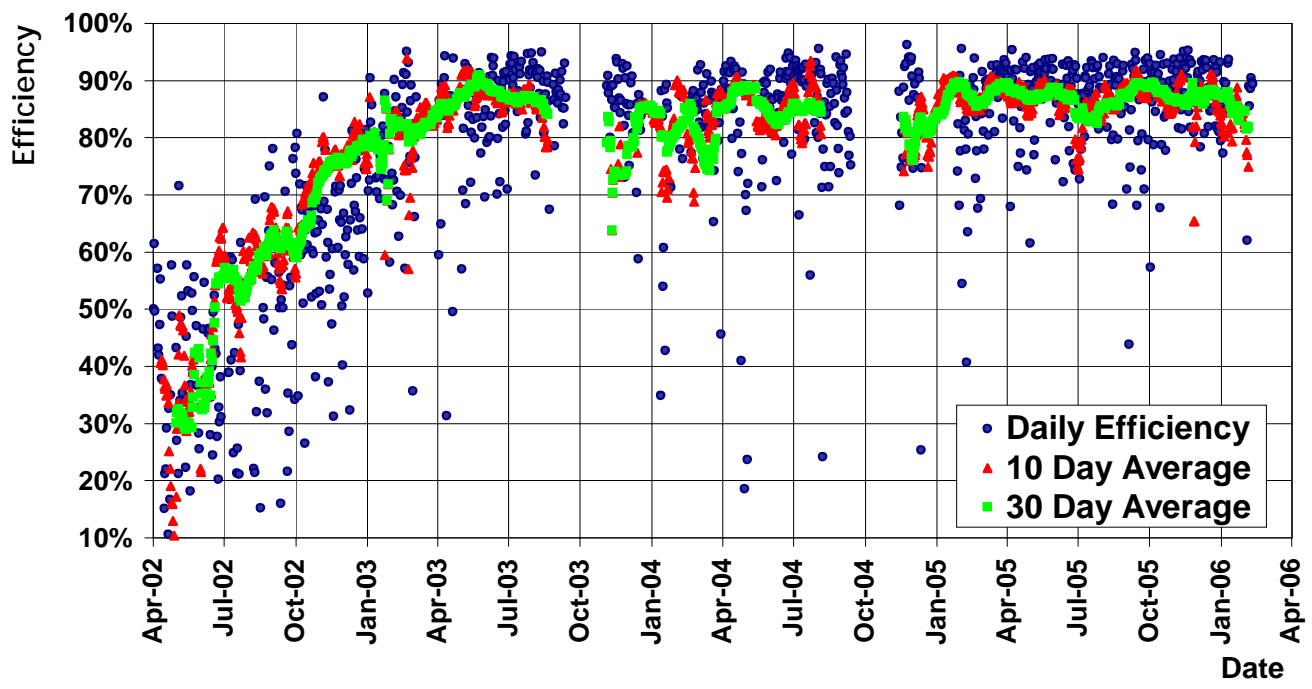

Figure 2.5: The data-taking efficiency of the DØ detector during Run IIa [31].

A right handed co-ordinate system is used throughout this thesis. The $z$-axis is along the beampipe in the proton direction (Fig. 2.3), the $y$-axis is upwards, and the $x$-axis points towards the centre of the Tevatron. The angles $\phi$ and $\theta$ are the azimuthal and polar angles, and $r$ is the perpendicular distance from the beampipe.

\footnotetext{
${ }^{1}$ Data taking efficiency is defined as the ratio of the recorded to the delivered data.
} 
The pseudorapidity, $\eta=-\ln \left(\tan \left(\frac{\theta}{2}\right)\right)$, approximates the true rapidity in the limit that $\frac{m c^{2}}{E} \rightarrow 0$. Objects are also frequently matched in $\eta-\phi$ space using the 'cone' distance, $\mathrm{dR}=\sqrt{d \eta^{2}+d \phi^{2}}$.

\subsubsection{The Central Tracking Detector}

Good tracking, and by extension vertexing, is essential for a wide range of physics studies including Higgs, top, B, electroweak and new phenomena. The central tracking system, shown in Fig. 2.4, consists of a high precision silicon microstrip tracker (SMT) and a central fibre tracker (CFT) surrounded by a $2 \mathrm{~T}$ superconducting solenoid. The central tracking provides a primary vertex resolution of $35 \mu \mathrm{m}$, a $b$-tagging resolution of $15 \mu \mathrm{m}$ for central tracks with transverse momentum, $\mathrm{p}_{T},>10 \mathrm{GeV}$, and a momentum resolution of $2+0.15 p_{T} \%$.

\subsubsection{The Silicon Microstrip Tracker}

The SMT, shown in Fig. 2.6, provides tracking and vertexing for almost the full $\eta$ range of the calorimeter and muon detectors. The SMT detector is made from high precision silicon wafers arranged into three sub-detectors:

Barrels - There are 6 barrel detectors in total, 3 either side of the origin, each made from 4 concentric layers of double sided rectangular silicon wafers providing information in $r-\phi$ (p-side) and $r-z$ (n-side). There are 12 wafers in layers 1 and 2 , and 24 in layers 3 and 4 . The barrel detectors cover $2.7 \mathrm{~cm}<r<10.5 \mathrm{~cm}$ and $|z|<38 \mathrm{~cm}$, providing tracking in the region $|\eta|<2.4$.

F-Disks - There are 12 F-disks, 6 cap the high $|z|$ of each barrel and 2 triplets of F-disks spaced 5, 10 and $15 \mathrm{~cm}$ from either end of the barrel detectors. Each F-disk is constructed from 12 double sided wedge shaped silicon modules.

H-Disks - The H-disks are designed for high $\eta$ coverage and 2 doublets are placed $1 \mathrm{~m}$ either side of the barrel detector. Each H-disk is made from 24 wedges, each wedge is constructed from back to back single sided silicon modules. The H-disk extend to coverage of the SMT tracking in the forward region up to $|\eta|<3.0$.

The pitch of the strips is predominantly $50 \mu \mathrm{m}$ (p-side) and $62.5 \mu \mathrm{m}$ (n-side), with hit resolutions of $\sim 10 \mu \mathrm{m}$. The signal to noise ratio varies from 12:1 to 18:1 depending on the detector type. Towards the end of Run IIa $\sim 15 \%$ of the barrel, $\sim 5 \%$ of the F-disk and $\sim 15 \%$ of the H-disk silicon modules were disabled [32]. 


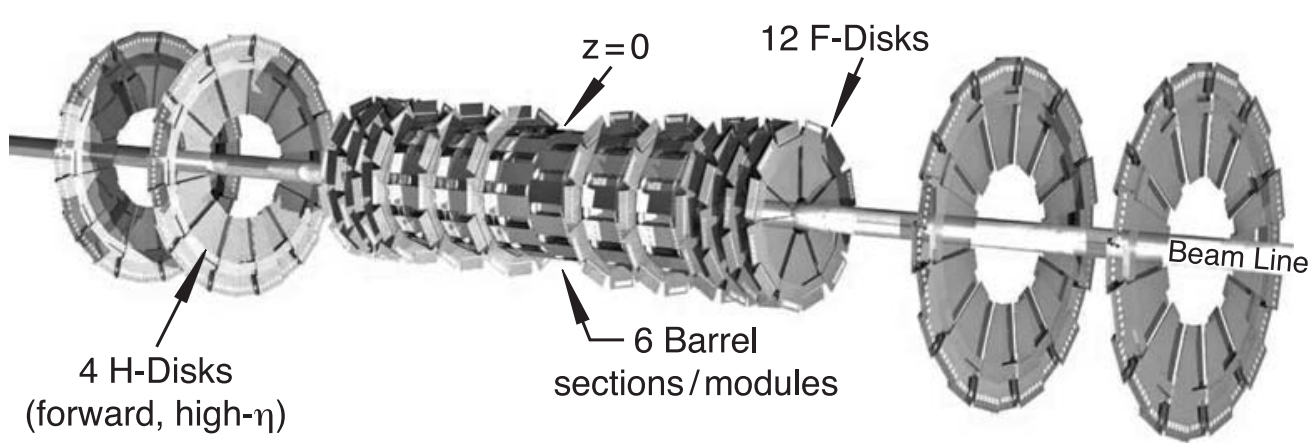

Figure 2.6: The silicon microstrip tracker.

As part of the Run IIb upgrade an additional layer of silicon has been added to the $\mathrm{D} \varnothing$ detector, referred to as Layer- 0 . Layer- 0 is located inside the current barrel detector at a radius of $1.6 \mathrm{~cm}$, and as well as recovering any performance loss due to radiation damage, will also improve the $b$-tagging resolution by a factor of 2 for low $\mathrm{p}_{T}$ tracks [33].

\subsubsection{The Central Fibre Tracker}

The CFT provides tracking in the region $|\eta|<1$.6. Fibres are arranged in layers of concentric circles around the beam pipe. A doublet layer is made from 256 fibres divided into two layers of 128 fibres offset by half the diameter of a fibre. A 'super layer' is made from two doublet layers, one parallel to the beam pipe providing $r-\phi$ information and one at an angle of $\pm 2^{\circ}$ providing $r-z$ information. There are 8 super layers covering $20 \mathrm{~cm}<r<52 \mathrm{~cm},|z|<1.26 \mathrm{~m}$ in the outer 6 super layers and $|z|<0.88 \mathrm{~m}$ in the two inner super layers.

The scintillating fibres are constructed from doped polystyrene surrounded by a double cladding with a total diameter of $835 \mu \mathrm{m}$. The scintillating fibres are connected to clear fibre waveguides which carry the light to visible light photon counters (VLPC) where the light is converted to an electrical signal. The VLPCs have a fast response time, a quantum efficiency of greater than $75 \%$ and a high gain of 22,000 - 65,000. The central fibre tracker has $\sim 99.0 \%$ of the VLPCs functional [32].

\subsubsection{The Solenoid}

The $2 \mathrm{~T}$ superconducting solenoid was designed to optimise the momentum resolution. The size of the solenoid was determined by the size of the calorimeter and the tracking requirements and it is $2.73 \mathrm{~m}$ in length, $1.42 \mathrm{~m}$ in diameter, and is 1.1 radiation lengths thick. The field is uniform to $0.5 \%$ within the tracking volume. 


\subsubsection{The Pre-Shower Detectors}

The pre-shower (PS) functions as a calorimeter as well as a tracking detector, and is used for electron identification, background rejection and aides in matching central tracks to calorimeter clusters. The pre-showers are triangular scintillator tiles placed in between the solenoid and the central calorimeter for the central pre-shower covering $|\eta|<1.3$, and attached to the inner faces of the end calorimeters for the forward pre-shower covering $1.5<|\eta|<2.5$ (see Fig. 2.4).

\subsubsection{The Calorimeter}

The calorimeter, shown in Fig. 2.7, measures the energy of electrons, photons and jets, as well as the transverse energy balance. The calorimeter is a liquid argon sampling calorimeter and consists of three sections: the central calorimeter (CC) which provides coverage up to $|\eta| \sim 1$ and two end caps (EC) which provide coverage up to $|\eta| \sim 4$.

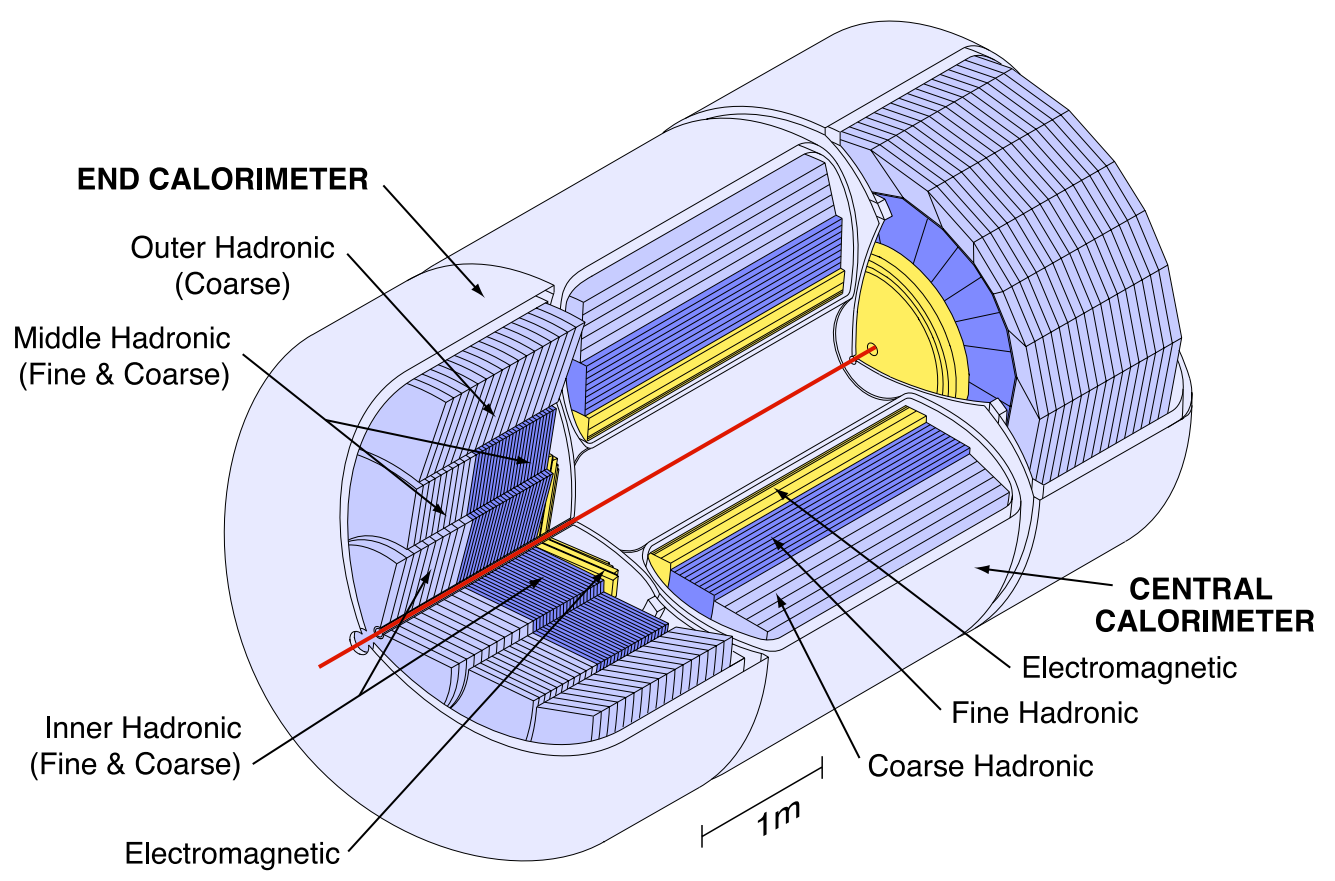

Figure 2.7: The central and two end cap calorimeters.

The calorimeter uses different absorbers in different sections: the electromagnetic (EM) layers use 3-4 mm thick uranium, the fine hadronic $(\mathrm{FH})$ layers use $6 \mathrm{~mm}$ thick uranium and the coarse hadronic $(\mathrm{CH})$ layers use $46.5 \mathrm{~mm}$ thick plates of copper (CC) or stainless steel (EC). The EM layers are designed to collect most of the EM energy, the FH layers most of the hadronic energy and the $\mathrm{CH}$ layers any 
leakage. The EC is split into three sections, each of which has a different make-up of absorber layers, starting from the beampipe these are referred to as the inner, middle and outer sections (see Fig. 2.7). The number of layers in the CC and EC and their electromagnetic $\left(\mathrm{X}_{0}\right)$ and nuclear absorption lengths $\left(\lambda_{A}\right)^{2}$ are:

Central Calorimeter - The CC has 4 EM layers $\left(20 \mathrm{X}_{0}\right), 3 \mathrm{FH}$ layers $\left(3.1 \lambda_{A}\right)$ and $1 \mathrm{CH}\left(3.2 \lambda_{A}\right)$ layer.

End Cap Calorimeter - The EC has 4 EM layers (inner: $21.4 \mathrm{X}_{0}$ ), $4 \mathrm{FH}$ layers (inner: $4.4 \lambda_{A}$, middle: $3.6 \lambda_{A}$ ) and $1 \mathrm{CH}$ layer (inner: $4.1 \lambda_{A}$, middle: $4.6 \lambda_{A}$, outer: $\left.6.0 \lambda_{A}\right)$.

Each layer is subdivided into calorimeter cells which have an area of $\Delta \eta \times \Delta \phi \sim$ $0.1 \times 0.1$, except the third EM layer (where the maximum EM energy deposit occurs) which has a finer granularity of $\Delta \eta \times \Delta \phi \sim 0.05 \times 0.05$, and all cells at $\eta>3.2$ which have an area $\Delta \eta \times \Delta \phi \sim 0.2 \times 0.2$.

The calorimeter is currently operating with $99.9 \%$ of the 55,000 channels operational, and has had a full cell-by-cell EM and hadronic calibration [32]. The resolution of the calorimeter for electrons and pions was measured from test beam data to be [34]:

$$
\begin{aligned}
\mathrm{EM}: & \frac{\sigma_{E}}{E}=\frac{0.15}{\sqrt{E}}+0.003 \\
\pi^{ \pm}: & \frac{\sigma_{E}}{E}=\frac{0.45}{\sqrt{E}}+0.04 .
\end{aligned}
$$

\subsubsection{The Intercryostat Detector}

The region $0.8<|\eta|<1.4$ has incomplete coverage from the calorimeter and substantial unsampled material; the intercryostat (ICR) detector is designed to provide additional sampling in this region and improve the energy resolution. The ICR covers the region $1.1<|\eta|<1.4$ and consists of scintillating tiles mounted on the cryostat walls of the EC calorimeters (see Fig. 2.4). There are 16 tiles in total, each of which is subdivided into twelve readout tiles of size $\Delta \eta \times \Delta \phi \sim 0.1 \times 0.1$.

\footnotetext{
${ }^{2}$ The absorption length is the mean free path of a particle before undergoing a non-elastic interaction in a given medium.
} 


\subsubsection{The Muon Detector}

The muon system is the outermost layer of the detector and provides efficient muon triggering and identification up to $|\eta| \sim 2$. There are two sections of the detector, the central muon system up to $|\eta|<1$ and the forward muon system in the region $1<|\eta|<2$, each of which has a series of drift tubes and scintillators.

Each system has three layers of drift tubes; Layer A lies inside a $1.8 \mathrm{~T}$ solid iron toroid magnet and $\mathrm{B} / \mathrm{C}$ which lie outside. The central muon system uses proportional drift tubes (PDT) and the forward system mini drift tubes (MDT). Alayers have 4 decks of drift tubes and the B/C-layers have 3. The PDTs are broken down into cells of $10.1 \mathrm{~cm}$ and have a maximum drift time of $500 \mathrm{~ns}$. The MDTs are subdivided into cells of $1 \mathrm{~cm}$ and have a maximum drift time of $60 \mathrm{~ns}$.

The central muon system has two scintillator counters, one before Layer A and one after Layer $\mathrm{C}$. The forward muon system has three scintillator counters located before Layers A and C, and one after Layer B. The scintillator counters are used to trigger on muons, and to provide accurate timing information for track reconstruction in the drift chambers.

The central muon system has $98.6 \%$ of the $8 \mathrm{k}$ tubes active and $99.8 \%$ of the scintillator counters active. In the forward region $99.7 \%$ of the $50 \mathrm{k}$ wires are active and $99.9 \%$ of the 4608 scintillator counters are active. The muon system is stable over time to $\sim 1 \%$ [32]. The scintillator counters have a time resolution of $\sim 2 \mathrm{~ns}$, and both the PDT and MDT have a hit resolution of $\sim 1 \mathrm{~mm}$. The momentum resolution of the muon system is defined by the central tracking system for muons with momentum up to $100 \mathrm{GeV}$, after this the muon systems improve the resolution. The central muon system has a momentum resolution of $0.36(p-3.1) / p \oplus 0.03 p \%$ (where $p$ is in $\mathrm{GeV}$ ) and the forward muon system of $\sim 20 \%$.

\subsubsection{Luminosity Monitor}

The luminosity monitor is used to determine the luminosity $(\mathscr{L})$ at the $\mathrm{D} \varnothing$ interaction point by detecting inelastic $p \bar{p}$ collisions. Two arrays of 24 plastic scintillation counters are placed at $z= \pm 140 \mathrm{~cm}$, just in front of the EC calorimeter and in the region between the beampipe and the frontal pre-shower detectors, covering the region $2.7<|\eta|<4.4$ (see Fig. 2.4).

The luminosity is calculated from the average number of inelastic $p \bar{p}$ collisions per bunch crossing $\left(\bar{N}_{L M}\right)$, using the following formula:

$$
\mathscr{L}=\frac{f \bar{N}_{L M}}{\sigma_{L M}},
$$


where $f$ is the bunch crossing frequency and $\sigma_{L M}$ is the cross section of inelastic $p \bar{p}$ collisions taking into account both the acceptance and efficiency of the luminosity monitor.

\subsubsection{The Trigger}

The vast majority of all collisions at a hadron collider result in what are considered to be 'background' events. The production cross section for background processes is several orders of magnitudes larger than that for most signal processes. A trigger is designed to retain the signal events to be kept from this overwhelming background.

The trigger at $\mathrm{D} \varnothing$ has three layers of increasing complexity referred to as Level 1 (L1), Level 2 (L2) and Level 3 (L3). Each level has increasingly sophisticated event reconstruction, and an event will proceed through each of the trigger levels dependent on conditions being meet. The trigger rates at each level of the trigger and the latency are shown in Table 2.2. A program called COOR handles the overall coordination and control of the trigger system, and interacts directly with the trigger framework (which makes the L1 and L2 trigger decisions) and the data acquisition supervisor (for L3). An overview of the DØ trigger and data acquisition systems is shown in Fig. 2.8.

\begin{tabular}{|c|c|c|}
\hline & Rate & Latency \\
\hline Collisions & $1.7 \mathrm{MHz}$ & $\mathrm{n} / \mathrm{a}$ \\
\hline $\mathrm{L} 1$ & $1.6 \mathrm{kHz}$ & $3.6 \mu \mathrm{s}$ \\
\hline $\mathrm{L} 2$ & $800 \mathrm{~Hz}$ & $\sim 100 \mu \mathrm{s}$ \\
\hline $\mathrm{L} 3$ & $50 \mathrm{~Hz}$ & $\sim 150 \mathrm{~ms}$ \\
\hline
\end{tabular}

Table 2.2: Approximate trigger rates and latency for the three trigger levels.

\subsubsection{Level 1}

The L1 trigger is hardware based and uses a reduced form of the detector readout, information from the CFT, PS, Muon and Calorimeter sub-detectors is used. The calorimeter trigger decision is based upon the transverse energy, $E_{T}$, sum in $\Delta \eta \times$ $\Delta \phi \sim 0.2 \times 0.2$ towers of calorimeter cells called 'trigger towers'. The trigger towers can either be summed to give a total $E_{T}$ for the calorimeter or the number of trigger towers with an $E_{T}$ deposit above a threshold can be calculated and used to trigger events. The track trigger groups fibres from the CFT into $4.5^{\circ}$ sections, and 


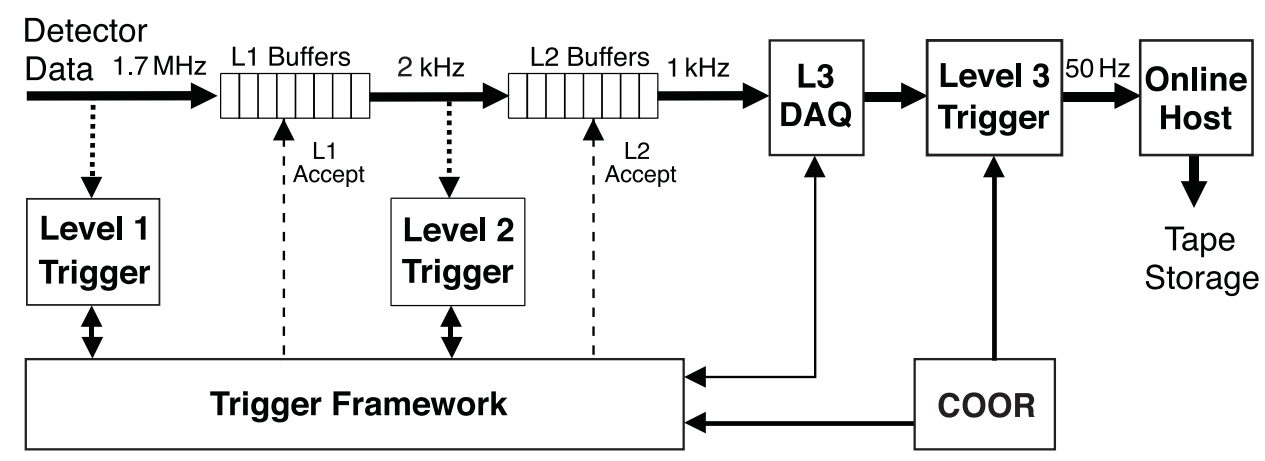

Figure 2.8: The D $\varnothing$ trigger and data acquisition systems.

matches the hits to pre-programmed patterns representing different $\mathrm{p}_{T}$ thresholds. The tracks are also matched to hits in the pre-shower detector. The muon trigger looks for patterns consistent with muons using the scintillator hits, wire hits and the tracks from the track trigger.

If a trigger has passed, then the 'trigger bit' corresponding to that trigger is said to have 'fired' and the event is passed into a buffer for evaluation by the L2 trigger.

\subsubsection{Level 2}

The L2 trigger is firmware based and uses the L1 data, with the addition of the SMT data. The data from each sub-detector is processed to produce physics objects (such as muons, jets, tracks, electrons) which are then passed onto a global processor. The global processor creates global physics objects from one or more of the sub-detector physics objects (such as matching a track to an EM object to make an electron object), and allows event wide correlations between all the L2 objects to be tested.

Each L1 trigger bit corresponds to one or more L2 triggers. If a L1 trigger bit is set, each of the corresponding L2 triggers is processed. This involves generating all the physics objects required by the trigger, and checking them against the thresholds defined in the trigger. If any of the L2 trigger thresholds pass then the event is passed on to the L3 trigger.

\subsubsection{Level 3}

The L3 trigger is fully software based and reconstructs events based upon the full detector readout on a farm of standard PCs running Linux. Events are reconstructed using two types of tool:

Unpacking Tools - Read in the raw data and convert it into a form usable by the physics tools. 
Physics Tools - Reconstruct physics objects in the detector using the output from the unpacking tools and/or other physics tools.

Each tool has a set of parameters which are defined at runtime to control the algorithm's behaviour. A limited number of parameter sets are defined for each tool, referred to as the tool's 'refset', these are the only instances of the tool which triggers can call.

Each L2 trigger bit corresponds to one or more L3 triggers. If a L2 trigger bit is set, the corresponding L3 triggers are processed. Each L3 trigger consists of a 'filter script' which contains one or more 'filters', and each 'filter' defines a condition. For a trigger to pass an event, each of the 'filters' in a filter script has to pass its condition. There are two kinds of 'filters':

Physics Object Filters - Compares the physics objects obtained from a physics tool to predefined thresholds. If the physics tool has been run by another tool it will return the already calculated physics objects, otherwise the tool will be executed on the event.

Relational Filters - Executes other filters and combines the filters' results.

If an event passes a trigger, then the event is written out to tape for processing by the offline reconstruction code. A collection of all the 'filter scripts' and the 'refsets' is referred to as a 'trigger list'.

\subsubsection{Analysis Tools}

After an event has been passed by the trigger system and written out to tape, it will undergo a full event reconstruction on the offline CPU farm at Fermilab. The 'offline' reconstruction code will typically take $\sim 10$ s per event at an instantaneous luminosity of $60 \times 10^{32} \mathrm{~cm}^{-2} \mathrm{~s}^{-1}$ on a $2.4 \mathrm{GHz} \mathrm{CPU}$. Section 2.2.6.2 contains a brief overview of the offline reconstructed physics objects used throughout this thesis, and should be referred to when relevant. Section 2.2.6.3 contains an introduction to $b$-tagging, and the $b$-tagging tools available at $\mathrm{D} \varnothing$ which are frequently used in this thesis. 


\subsubsection{Reconstruction Code Versions}

The various versions of $\mathrm{D} \emptyset$ code which are used to reconstruct the offline physics objects are referred to by the terminology pXX, where the XX is an integer which is incremented for each new major release of the code. Two versions of the offline reconstruction code are used in this thesis, p14 and p17. All the work in this thesis, apart from the latest version of the analysis, were reconstructed with the p14 version of the code.

\subsubsection{Physics Objects}

The following physics objects are used in this thesis:

Tracks - Tracks are reconstructed using a combination of three algorithms. Two of the algorithms search for potential track candidates, and the third algorithm propagates the track candidates through the full detector.

The first algorithm [35, 36] searches for tracks starting from seeds of 3 SMT or CFT hits. The seed tracks are then propagated through the SMT and CFT detectors and at each layer a new seed track is created for every hit within the predicted trajectory. The second algorithm [37] uses a histogram based Hough transformation [38] to locate tracks.

Both the track finding algorithms are run by default, and all the track candidates from both algorithms are ranked together according to quality criteria. All the tracks which fulfil certain quality criteria are kept and the tracks are fitted using the $3^{\text {rd }}$ algorithm [39], based on a Kalman Track Fitter, which propagates the candidates through the detector taking account of the variations in the magnetic field, multiple scattering and energy loss [40].

Primary Vertex - A 2-pass probabilistic primary vertex (PV) algorithm was used to reconstruct PVs [41], except for the latest p17 version of the analysis which used an adaptively fitted vertex [42].

Jets - Calorimeter jets are reconstructed using a cone jet algorithm [43] of radius 0.5 in $\eta \times \phi$ space. Jet energy scale (JES) corrections are applied to jets to correct the energy of the jet to account for the calorimeter response to the hadronic jet, the fraction of the energy actually contained with the jet cone, and for other sources of energy within the calorimeter [44]. 
Flavoured Jets - In Monte Carlo (MC) events jets were identified as being $b$-jets if they had a $b$-hadron within $\mathrm{dR}<0.5$ of the jet. $c$-Jets had a $c$-hadron but no $b$-hadron. $u d s$-Jets or fake jets, which included gluon jets, had no heavy flavour at all present in the jet.

Track Jets - Track jets are formed from the tracks found within $\mathrm{dR}<0.5$ of a seed track. All tracks used in a track jet must have at least 2 SMT hits, the seed track must have $\mathrm{p}_{T}>1.0 \mathrm{GeV}$, and a track jet must consist of at least two tracks [46].

Taggable Jets - A taggable jet is a calorimeter jet matched to a track jet within a cone of $\mathrm{dR}<0.5$. The taggability definition is designed to select a sample of jets which satisfy basic tracking criteria [45].

Muons - All muons used in this thesis were required to be Medium muons (have at least two wire hits and a scintillator hit in both the A and BC layers), have a $p_{T}>4 \mathrm{GeV}$ and be matched to a track with $\chi^{2}<100[47]$.

\subsubsection{3 $b$-Tagging at $\mathrm{D} \varnothing$}

A $b$-jet, as shown in Fig. 2.9, can be identified by its relatively long lifetime and/or the presence of a high $\mathrm{p}_{T}$ lepton ${ }^{3}$. A track's impact parameter (IP) is defined as the perpendicular distance from the track to the PV, at the track's distance of closest approach to the PV. The IP is negative, if the track crosses the jet axis on the opposite side of the PV with respect to the jet. There are five tools available at D $\varnothing$ to identify ('tag') whether a jet was produced from a $b$ or not. Three use lifetime information, one uses lepton information, and the fifth is a NN based $b$-tagging tool. The NN b-tagging tool, which was developed as part of this thesis, is now the official DØ b-tagger and is outlined in detail in Chapter 4 . There follows a brief description of the other four $b$-tagging tools:

Counting Signed Impact Parameters (CSIP) Tagger [48] - Counts the number of tracks identified in a jet (tracks are matched to jets if they have a $\mathrm{dR}<0.5)$ which have a large IP significance ${ }^{4}$ with respect to the primary vertex. Events must have at least 3 tracks with an IP significance greater than 2 or 2 tracks with an IP significance greater than 3 to be considered tagged.

\footnotetext{
${ }^{3} \mathrm{~A} b$-jet will decay semi-leptonically $\sim 10 \%$ of the time.

${ }^{4}$ The significance of a measurement is defined as the measurement divided by its error.
} 
Jet Lifetime Probability (JLIP) Tagger [49] - Combines the impact parameter information from all the tracks identified in a jet into one variable called the Jet Lifetime Probability (JLIP Prob). JLIP Prob is the probability that all tracks originate from the primary vertex. The closer to zero the more likely that the jet originated from a $b$. The current operating points for the tagger range from $0.002\left(\right.$ Very Tight $\left.{ }^{5}\right)$ to $0.04\left(\right.$ Super Loose $\left.^{6}\right)$.

Secondary Vertex Tag (SVT) Tagger [50] - Uses tracks which are significantly displaced from the primary vertex to reconstruct secondary vertices (SV). A jet is considered tagged if a secondary vertex is located within $\mathrm{dR}<0.5$ of the jet. Cuts on the decay length significance (DLS) of secondary vertices range from 5 (Loose) to 7 (Tight).

Soft Lepton Tag (SLT) Tagger [51] - A jet is tagged if a muon is matched to the jet within $\mathrm{dR}<0.5$.

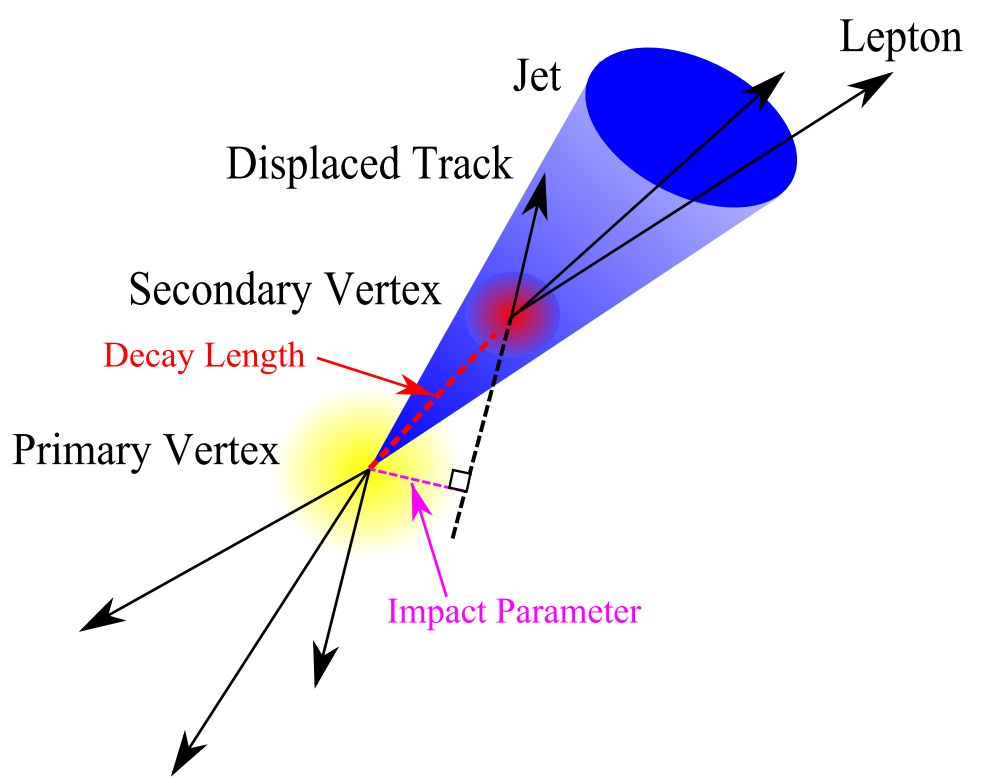

Figure 2.9: The different characteristics used to identify $b$-jets: a displaced secondary vertex, displaced tracks with large impact parameters and high $\mathrm{p}_{T}$ leptons.

\footnotetext{
${ }^{5}$ Very Tight: Low $b$-efficiency, low fake rate.

${ }^{6}$ Super Loose: High $b$-efficiency, high fake rate.
} 


\section{Chapter 3}

\section{Level 3 Trigger}

\subsection{Introduction}

This chapter records the Level 3 (L3) trigger work undertaken as part of this thesis. Section 3.2 describes the L3 tools used. Section 3.3 describes improvements to the tracking, Section 3.4 covers the development of the new L3 trigger terms for the Higgs multi-jet triggers and Section 3.5 discusses a new secondary vertex tagger (SVT) b-tagging tool. The author is solely responsible for the work documented in Sections 3.3 and 3.4; Section 3.5 was carried out with assistance from a fellow $\mathrm{PhD}$ student.

\subsection{Tools}

\subsubsection{Unpacking Tools}

Unpacking tools read in the raw data and convert them into a form usable by the L3 'physics' tools, which reconstruct physics objects in the detector. L3 unpacking tools exist for the SMT, CFT, calorimeter, pre-shower and muon detectors.

\subsubsection{Tracking}

The SMT [52, 53] and CFT [54] unpacking tools read in the raw data from the SMT and CFT sub-detectors and construct clusters which are used to reconstruct tracks. The position of a cluster represents a point where a charged particle passed through the detector. At L3 clusters are constructed in the x-y (axial) and z (stereo) planes.

Clusters in the SMT are formed by joining together consecutive 'hit' strips in the detector. Strips are considered 'hit' if, after pedestal subtraction, they have an 
ADC count above the predefined strip threshold. Clusters are kept if their total ADC count is above a predefined cluster threshold. The position of a cluster is calculated from the weighted mean of the strip positions with the strips weighted by their charge.

In the CFT clusters are formed by joining together consecutive fibres which, after pedestal subtraction, have an ADC count above a predefined fibre threshold. Clusters are kept if their total ADC count is above the predefined cluster threshold. The position of a cluster is calculated from the weighted mean of the fibre positions with the fibres weighted by their light yield.

The tracking tool $[55,56]$ uses clusters from the SMT and CFT unpacking tools to reconstruct tracks. The tracking tool has a very tight time budget and is limited to $\sim 100 \mathrm{~ms} /$ event. Consequently the L3 tracking tool is simpler than the tracking in the offline reconstruction code.

The tracking algorithm first searches for axial tracks. Potential tracks are created between all pairs of hits in the outer two layers of the CFT and the tracks are kept if they have a $\mathrm{p}_{T}$ greater than a predefined threshold. The potential tracks are propagated through the remaining axial layers of the CFT and SMT identifying clusters consistent with the track's predicted position at each layer. New potential tracks are created for each potential cluster if the addition of the cluster increases the $\chi^{2}$ of the track by less than 10. Any track which has missed more than 1 layer is discarded. Of the remaining tracks only the 3 or 4 tracks (depending on the layer) with the best quality are kept. After all the layers have been traversed the tracks which share more than 2 hits are identified and the worst quality track discarded. The axial tracking parameters are calculated using a simplified circle fitting algorithm [57].

Stereo tracking is carried out for each axial track using a fast histogramming method based on the Hough transformation [38]. Any stereo cluster lies on an infinite number of possible stereo tracks. If the parameters of all these potential tracks are plotted in $Z_{0}-\tan \lambda^{1}$ phase space, they will map out a straight line of all the possible parameter values a line through that cluster could take; this line is referred to here as a 'parameter line'. The parameter lines from all the stereo clusters of a track will intersect at the point which represents the stereo parameters of the track. A quick method to find the point of intersection of several parameter lines is to fill a 2 dimensional histogram with the parameter lines from all the stereo clusters associated to an axial track. The most populated bin in the histogram

\footnotetext{
${ }^{1} Z_{0}$ and $\tan \lambda$ are the two parameters which describe a helix shaped track in the $z$ direction.
} 
will normally correspond to the parameters of the correct stereo track. The stereo clusters in that bin are then used in a fast linear sequential fitter to calculate the best stereo track fit.

Due to the simplified algorithm used at L3 the tracking is less accurate, efficient and pure than the offline tracking tool; however it does run one hundred times faster.

\subsubsection{Vertexing}

The vertexing tool [58] uses the tracks to reconstruct the hard scatter primary vertex (PV). The $z$-vertex is reconstructed first by binning the $z$ values of all the tracks at the distance of closest approach to $(0,0)$ in a $\mathrm{p}_{T}$ weighted histogram. The $z$ value of the $\mathrm{PV}$ is determined using the tracks in the two adjacent bins with the highest population. Tracks which are consistent with the $z$-vertex are then used in an impact parameter (IP) minimisation technique, using the beam spot as an additional constraint, to calculate the most probable location of the $x-y$ vertex. The vertexing has a resolution of $\sim 44 \mu \mathrm{m}$ in $\mathrm{x}-\mathrm{y}$ and $\sim 1.9 \mathrm{~mm}$ in $\mathrm{z}$.

\subsubsection{Jets}

The jet tool [59] uses calorimeter clusters from the calorimeter clustering tool [60] to construct 0.5 radius cone jets. The jets are similar to the offline reconstructed jets [61] but constructed without jet splitting/merging to save time.

\subsubsection{Impact Parameter $b$-tagging Tool}

The IP b-tagging tool [62] uses the jets, tracks and PV as inputs to calculate track, jet and event probabilities based upon the signed IPs of the tracks. The IP significance of each track in a jet is combined into a jet probability, and the jet probabilities are combined into an overall event probability. The event probability is the probability that all the jets originated at the PV: a value of 0 indicates that it is highly likely that there is one or more $b$-jets in the event, a value of 1 that it is highly unlikely.

In addition to the IP $b$-tagging tool, a low $\mathrm{p}_{T}$ track IP filter also exists which allows triggering on individual tracks to identify low $\mathrm{p}_{T} \mathrm{~B}$ physics events [63].

\subsubsection{Muons}

The L3 muon reconstruction code [64] is very similar to the offline muon reconstruction code [47], although fewer potential tracks in the muon detector are tested to reduce the time taken. The various muon classifications are detailed below: 
Loose - Two out of the three following conditions are required:

Condition 1: 2 or more wire hits or at least one scintillator hit in the A-layer.

Condition 2: Two or more wire hits in the BC-layer

Condition 3: One or more scintillator hits in the BC-layer.

Medium - At least one wire and one scintillator hit in the A-layer and at least two wire and one scintillator hits in the BC-layer.

Tight - At least two wire and one scintillator hit in the A-layer and at least two wire and one scintillator hits in the BC-layer.

\subsection{Tracking and SMT Studies}

The tracking is a vital component of the trigger. The vertexing, $b$-tagging, lepton and jet finding tools all make use of the tracking, and almost all the physics triggers make use of at least one of these tools. Monitoring and improving the performance of the tracking tool is therefore an extremely important aspect of the work of the L3 algorithms group.

The author conducted studies into measuring, comparing and bench marking the performance of the tracking tool on data; these studies are briefly mentioned in Section 3.3.1. As a result of these studies it was found that the tracking tool's performance and the track quality (due to wrongly assigned SMT hits and a generally low number of SMT clusters per track) needed improving. The author's work in this area centred on studying the SMT unpacking tool and comparing its performance to the offline SMT clustering. The studies resulted in the identification of several problems and potential improvements. The author carried out the implementation of the fixes and improvements in early 2004, and they were approved by the Level 3 group. The new version of the L3 SMT unpacking tool was approved for use in June 2004. These studies are outlined in Section 3.3.2.

\subsubsection{Measurement of the Tracking Performance}

The performance of a tracking tool can easily be measured on Monte Carlo (MC) as the found tracks can be compared to the 'truth' tracks. However the tracking performance measured in $\mathrm{MC}$ tends to be over-optimistic in the number and quality of the found tracks; additionally the $\mathrm{MC}$ does not replicate many of the effects seen with data. It was therefore necessary to develop a method to measure and benchmark the tracking tool on data. 
The most accurate tracking information available for data is the offline tracks, these were used as the 'truth' tracks with respect to which the L3 efficiency and purity were measured. The L3 tracks were 'matched' to the offline tracks using two different methods.

The first method compares the online and offline CFT and SMT clusters assigned to the tracks. If clusters share a majority of the same strips or fibres, then the clusters are considered to be matched. The online track which shares the most clusters with an offline track is considered 'matched' to it. Tracks which share more than 8 axial CFT and 2 axial SMT hits are considered to be a 'good' match. This matching method was primarily used to diagnose problems with the L3 tracking and the clustering by directly comparing the clusters the L3 and offline tracks shared.

The second method compares the five track parameters which describe a track's helix shape. A cut of 15 on the $\chi^{2}$ agreement of the five tracking parameters is used to decide whether a L3 track is a 'good' match to an offline track or not. All other matched tracks are considered 'bad' matches and those not matched at all 'fake' tracks. The $\chi^{2}$ matching was used to measure the performance of the tracking.

There are hence three categories of tracks:

'Good' Matched Tracks - L3 tracks which are matched to an offline track.

'Bad' Matched Tracks - L3 tracks which are the best match to an offline track, but which fail the 'good' matching criteria.

'Fake' Tracks - L3 tracks which are not matched to any offline tracks.

The efficiency of the L3 tracking is defined as:

$$
\text { Efficiency }=\frac{\text { Number of Good Matched L3 Tracks }}{\text { Number of Offline Tracks }}
$$

and the purity as:

$$
\text { Purity }=\frac{\text { Number of Good and Bad Matched L3 Tracks }}{\text { Total Number of L3 Tracks }} .
$$

\subsubsection{SMT Problems}

SMT cluster and tracking comparisons were carried out using data run 189917 which is marked as 'good' in the runs quality database [65] for all the detector subsystems and was recorded at an instantaneous luminosity of $\sim 25 \times 10^{30} \mathrm{~cm}^{-2} \mathrm{~s}^{-1}$.

Comparisons of the performance of the L3 tracking tool and the offline tracking tool revealed several problems. Figure 3.1 shows the number of offline and L3 axial 
and stereo SMT clusters associated with each track. The L3 tracks typically had fewer clusters associated with each track. This is most apparent in the case of the stereo clusters where the L3 tracks had only $\sim 40 \%$ of the number of clusters of the offline tracks. Additionally, L3 and offline tracks which share 8 CFT clusters regularly had different SMT hits assigned to the tracks resulting in large discrepancies between the L3 and offline tracking parameters. The most likely explanation for these differences was a problem with either the L3 tracking, the clustering or both. A comparison of the number of clusters found per event is shown in Fig. 3.2. A large difference existed between the old L3 and offline clustering. The differences and their causes are discussed in the following sections along with other clustering issues and improvements implemented in the L3 SMT unpacking tool.
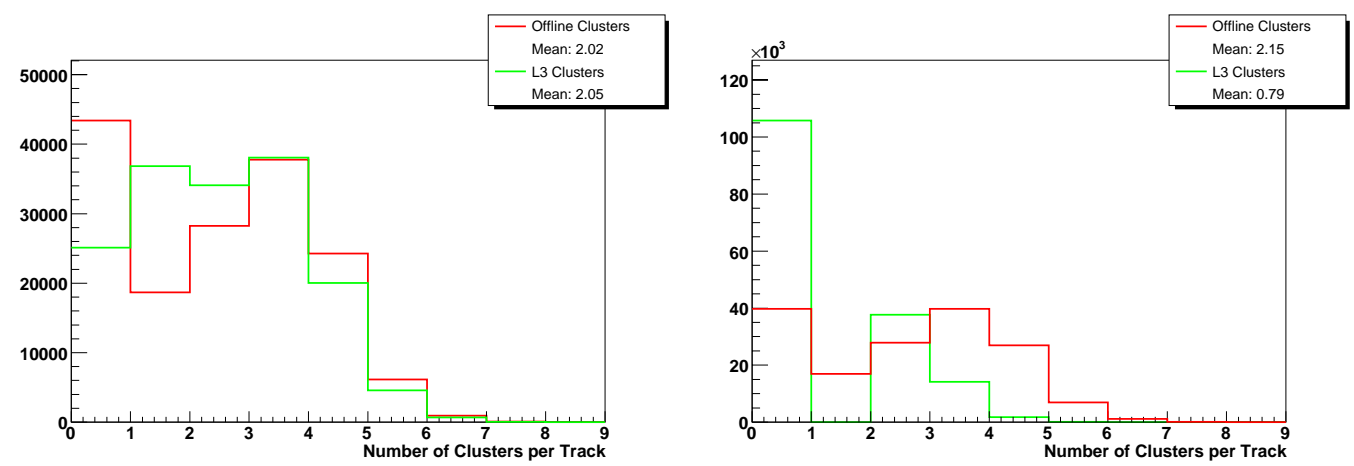

Figure 3.1: For data run 189917 the number of axial (left) and stereo (right) SMT clusters assigned to the L3 (green) and offline (red) tracking tools. The distributions were normalised to be of equal integral.
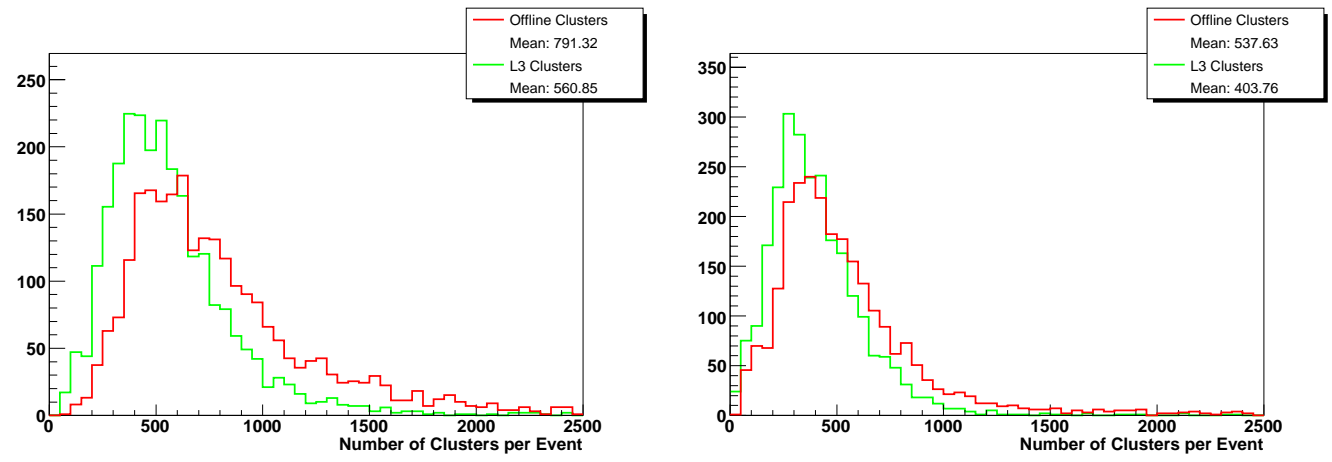

Figure 3.2: For data run 189917 the number of axial clusters (left) and stereo clusters (right) found by the L3 (green) and offline (red) SMT unpacking tools.

\subsubsection{1 'Hot' Silicon Vertex Chips}

The old L3 SMT unpacking tool regularly found 180+ clusters in some ladders, whereas the offline clustering would find only a couple of clusters. This problem was 
found to be due to malfunctions in the silicon vertex (SVX) chips, which read-out the SMT detector, resulting in the majority of the SMT strips being registered as 'hit'.

The number of hit strips per SVX chip is shown in Fig. 3.3 for data run 189917. The vast majority of SVX chips have a low hit occupancy. However, a small number of chips have a high occupancy, with the peak at 128 corresponding to SVX chips which have registered every strip as being hit. These problematic SVX chips will result in hundreds of (most probably fake) extra clusters if they are not discarded.

This problem had been identified and fixed by the offline clustering group, but the fix had not been propagated into the L3 SMT clustering code. The offline clustering group defined any SVX chip with $>25 \%$ occupancy in an event as a 'hot' chip, which should be rejected for that event only. This cut was also implemented in the L3 SMT unpacker.

The total number of clusters per event due to 'hot' SVX chips is shown in Fig. 3.4. An average of $\sim 340$ fake clusters per event are found in the data sample due to 'hot' SVX chips, this is almost a third of the average number of clusters per event $(\sim 1000)$.
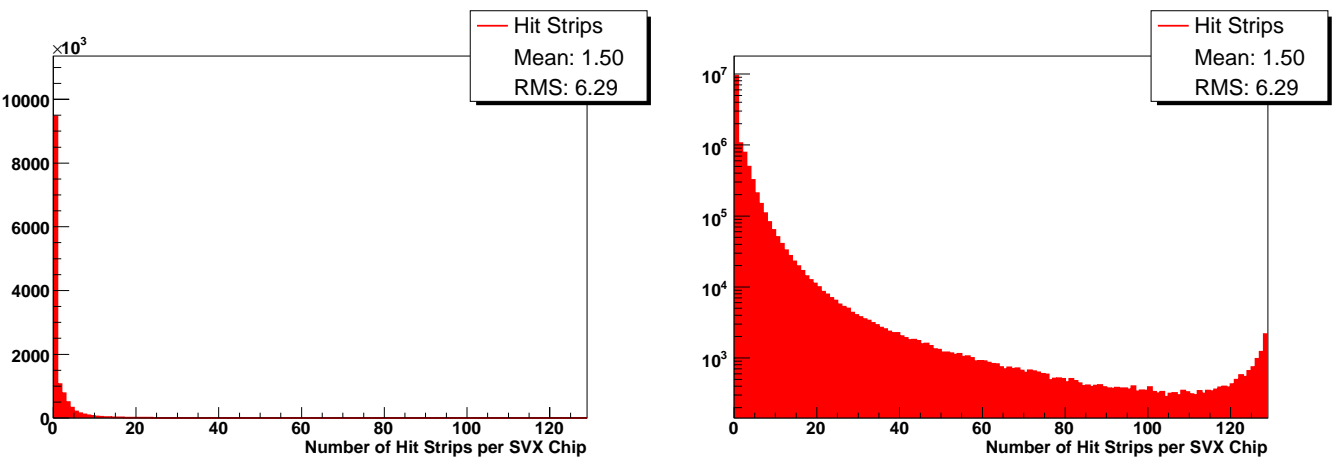

Figure 3.3: The number of hit strips in each SVX chip (left) and on a log scale (right) for 2500 events from data run 189917. Each SVX chip reads out 128 strips.

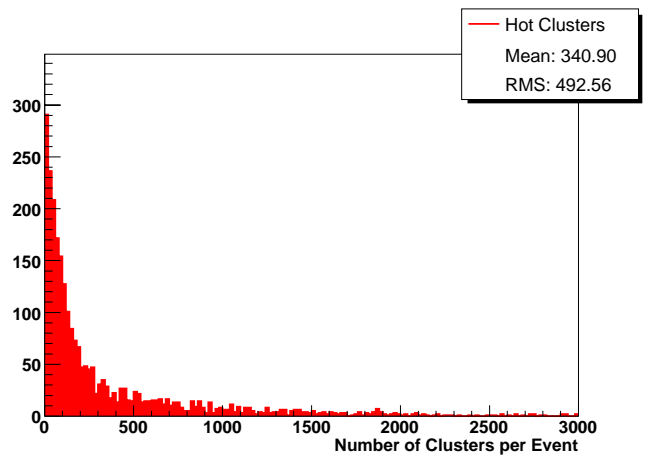

Figure 3.4: The number of clusters per event found due to 'hot' SVX chips. 


\subsubsection{Pedestal Values}

The L3 unpacking code found fewer clusters than the offline code. This was partly due to the higher strip and cluster thresholds. The thresholds used in the L3 unpacking code were therefore loosened, to increase the strip and cluster acceptance, by measuring the efficiency whilst maintaining a reasonable purity. The new threshold cuts are given in Table 3.1. The new cuts are set slightly looser than the offline code.

\begin{tabular}{|c|c|c|}
\hline \multirow{2}{*}{ SMT Unpacker } & \multicolumn{2}{|c|}{ Threshold (ADC) } \\
\cline { 2 - 3 } & Channel & Cluster \\
\hline Old L3 & 10 & 10 \\
L3 & 7 & 9 \\
Offline & 8 & 10 \\
\hline
\end{tabular}

Table 3.1: The threshold cuts used in the old L3, new L3 and offline SMT unpacking tools.

\subsubsection{Merging Clusters}

Due to hardware problems and radiation damage various strips in the SMT are 'dead' (pedestal less than 10 ADC counts) or 'noisy' (pedestal RMS more than 6 ADC counts). These strips are marked as such in the SMT calibration database, and any hits marked as 'dead' or 'noisy' have their ADC pedestal set to 999 to mask any output from the strip.

To account for 'noisy' and 'dead' strips, the old L3 SMT unpacker merged any two strips which were separated by an unhit strip. The new SMT unpacker takes a more sophisticated approach and only merges two strips together if they are separated by a 'dead' or 'noisy' strip. An example of this situation is shown in Fig. 3.5, which shows 5 consecutive strips in the SMT detector. The distribution of the number of clusters per event which contain a dead strip when using the new algorithm is shown in Fig. 3.6. The average number of affected clusters is $\sim 6$, which compared to the total number of clusters $(\sim 1500)$ is negligible. However, this algorithm will become more important as the SMT detector degrades due to radiation damage.

\subsubsection{Splitting Clusters}

Clusters of multiple strips may actually be overlapping clusters. The old L3 SMT unpacker would just consider overlapping clusters as one long cluster, with a cluster position somewhere in between the two actual clusters. A splitting algorithm was 


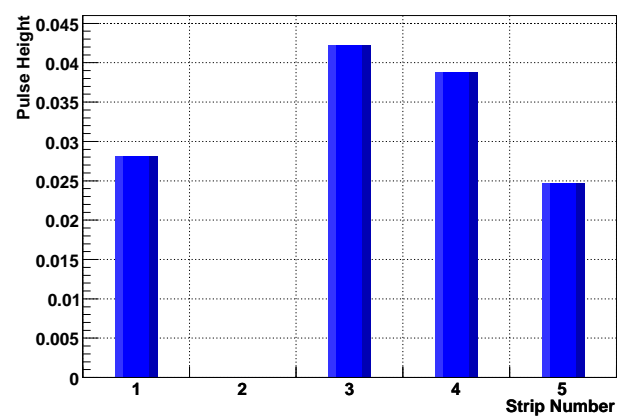

Figure 3.5: An example from a data event of consecutive strips in the SMT detector, all of which registered a hit except strip 2 which is a 'dead' or 'noisy' strip.

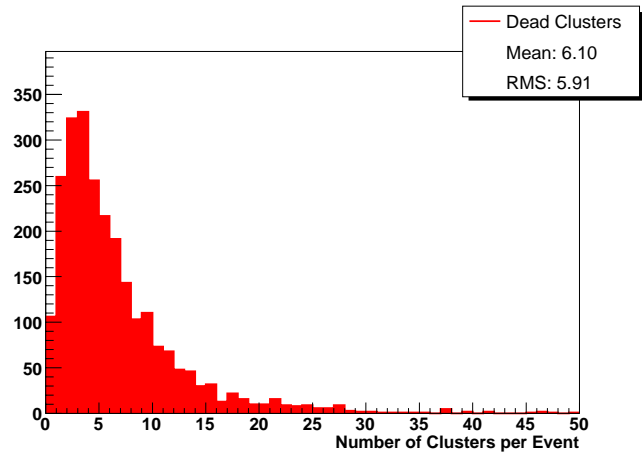

Figure 3.6: The number of merged clusters which contain a 'dead' or 'noisy' strip per event, based on 2500 events.

therefore developed and implemented in the L3 SMT clustering which identifies merged clusters and splits them into their component clusters.

The splitting algorithm is applied to any cluster which is constructed from 2 or more strips. Each strip is considered in turn and assigned to a 'level'. The 'level' is a number assigned to a group of strips which do not deviate by more than the average strip noise $( \pm 2.2 \mathrm{ADC}$ counts $[66,67])$. If a strip deviates by more than this from the first strip in that level, then the strip is considered to be in the level above or the level below. This is repeated for each strip in the cluster, until they have all been assigned to a level.

Once the strips are divided into different levels, the numbers and locations of the peaks and troughs (if any) are identified. A peak is a level which is higher than both its neighbouring levels; a trough is a level which is lower than both its neighbouring levels. Levels at the beginning or end of a cluster only need to be higher or lower than their one neighbouring level. If there is more than one peak in the cluster then the cluster is divided up into smaller clusters. The strips found in the trough between two peaks are considered to be the dividing point between the 
two clusters. If there are an even number of strips in the trough then the strips are divided equally between the two clusters. If there is an odd number of strips then the middle strip is divided into two with both clusters getting one strip with half the ADC count. Figure 3.7 shows an eleven strip cluster which has been divided into three clusters by the algorithm.

The total number of new clusters per event created due to the splitting algorithm is shown in Fig. 3.8, along with the number of clusters each split cluster was divided into. The average number of clusters created from splitting merged clusters is 126 per event, this equates to an additional $\sim 76$ clusters per event. The majority of split clusters form two new clusters.

\subsubsection{SMT Cluster Errors}

The old L3 SMT cluster errors were determined from a $t \bar{t}$ MC sample by examining the cluster residuals, namely the distance between the found cluster and the $\mathrm{MC}$ 'truth' cluster [53]. However as these errors were measured on MC samples they were an underestimate of the error on the cluster's position. The inaccurate errors assigned to the cluster's position was found to be one of the main contributions to the low number of SMT hits per track when compared to the offline tracks.

New errors were measured by examining the residuals between the cluster and L3 tracks in data run 189917, as shown in Fig. 3.9 for axial and stereo clusters for a selection of 'good' matched tracks.

The half width half maximum of the residual distributions was used to assign errors to the SMT clusters. The old and new errors are shown in Table 3.2. The new axial errors are a factor of $\sim 3$ larger, and the stereo errors a factor of $\sim 7$ larger. The new errors are a measurement of the spread of the SMT clusters around the tracks. The measured uncertainties contain a component from the displacement of the clusters with respect to their true position and a component from the simplified L3 tracking circle fit which will cause an additional displacement between the track and cluster positions.

\begin{tabular}{|c|c|c|}
\hline \multirow{2}{*}{ L3 Unpacking Tool } & \multicolumn{2}{|c|}{ Error $(\mu m)$} \\
\cline { 2 - 3 } & Axial & Stereo \\
\hline Old & 7.5 & 20 \\
New & 20 & 150 \\
\hline
\end{tabular}

Table 3.2: The errors assigned to the SMT hits in the new and old versions of the L3 SMT unpacking tool. 


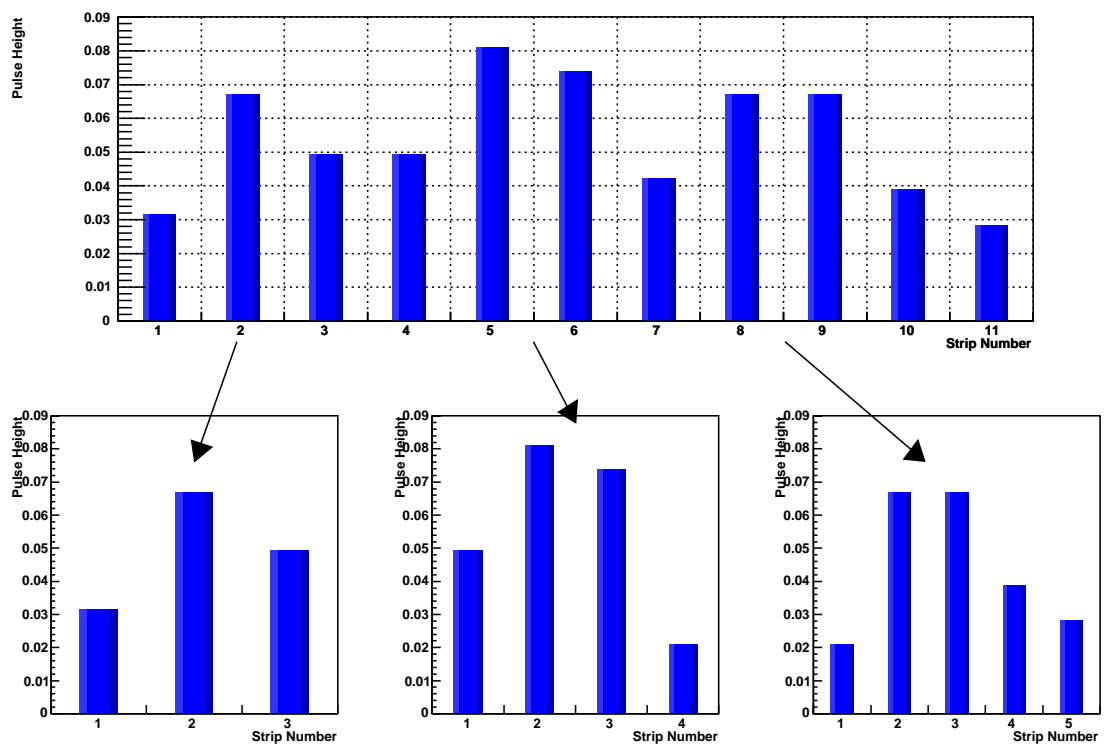

Figure 3.7: An example from a data event of consecutive strips in the SMT detector which are divided by the splitting algorithm. Three distinct peaks are found at strips $2,5+6$ and $8+9$, and two troughs are found at $3+4$ and 7 . The large cluster is therefore split into 3 smaller clusters corresponding to the three peaks. The strips in the troughs are split amongst the clusters. The first cluster gets strip 3 and the second strip 4 . The second and third clusters both get half of strip 7.
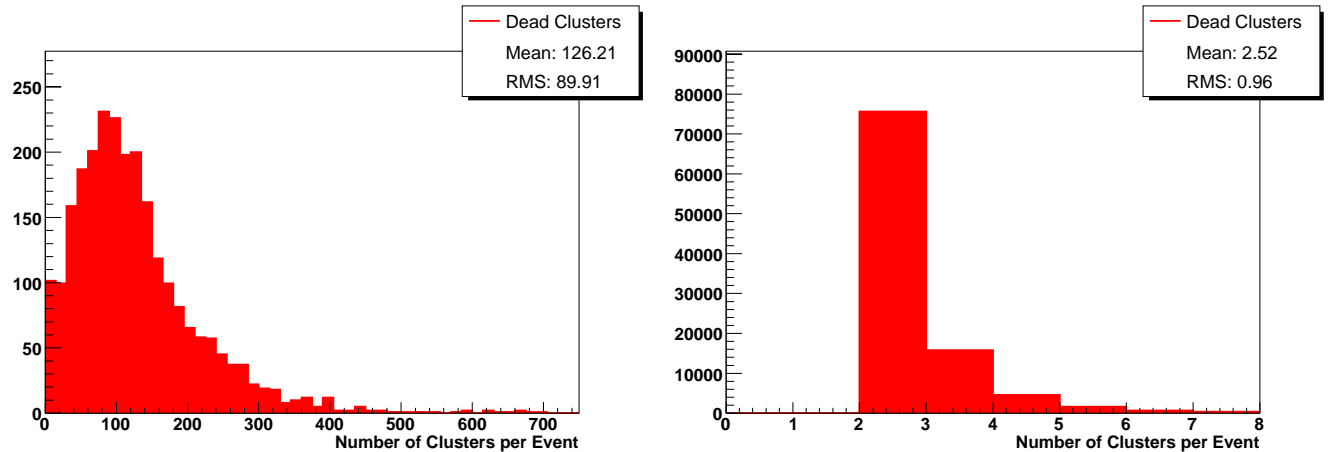

Figure 3.8: The number of clusters per event created by cluster splitting (left) and the number of clusters each split cluster produces (right) based on 2500 events. 

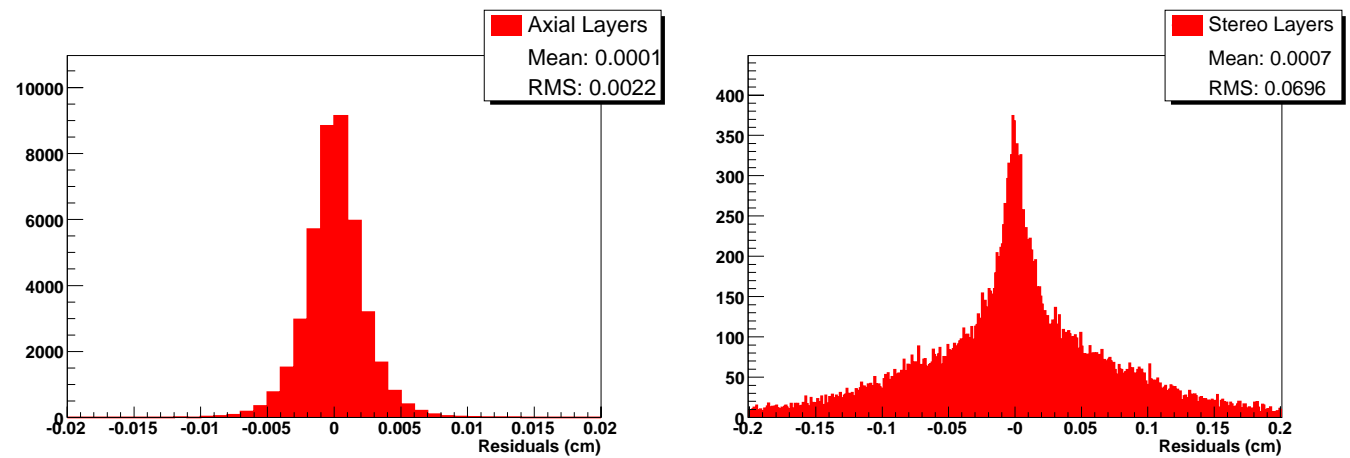

Figure 3.9: The residuals between the L3 tracks and the closest L3 cluster for the axial layers (left) and the stereo layers (right). The tracks were all required to have 8 CFT and at least 2 SMT hits matched to an offline track.

\subsubsection{Results}

\subsubsection{Number of Clusters}

The numbers of clusters found by the offline, the old L3 and the new L3 SMT unpacking tools are shown in Fig. 3.10. The number of clusters found by the new L3 SMT unpacking tool is now in much better agreement with the number found by the offline unpacking tool. The new clustering code actually finds slightly more clusters than the offline code due to the lower ADC threshold cuts and the splitting algorithm, which had no equivalent in the offline code. The cluster splitting algorithm has since been implemented in the offline clustering code.

\subsubsection{Tracking}

The efficiency and purity of the L3 tracking tool when using the old and new SMT unpacking tools is shown in Fig. 3.11. Both the tracking efficiency and purity are improved using the new L3 SMT unpacking tool. Absolute gains in efficiency of $\sim 5 \%$ and in purity of $\sim 1-2 \%$ at low $\mathrm{p}_{T}$ increasing up to $\sim 8 \%$ at high $\mathrm{p}_{T}$ are achieved. The L3 tracking efficiency and purity both fall with increasing $\mathrm{p}_{T}$, the reasons for this are still under investigation. The fall in efficiency is thought to be partly a consequence of the efficiency being derived from a comparison to the offline tracks. The high $\mathrm{p}_{T}$ tracks in data found by both trackers have a higher proportion of fake tracks than compared to the low $\mathrm{p}_{T}$ region, and the fake tracks found by the trackers are different due to the different fitting and track finding algorithm used. As the L3 tracking efficiency is calculated with respect to the offline tracks this leads to an apparent drop in efficiency.

Figure 3.12 shows the number of axial and stereo clusters associated with 'good' matched L3 tracks for the old and new unpacking tool. Increases of $\sim 10 \%$ and 

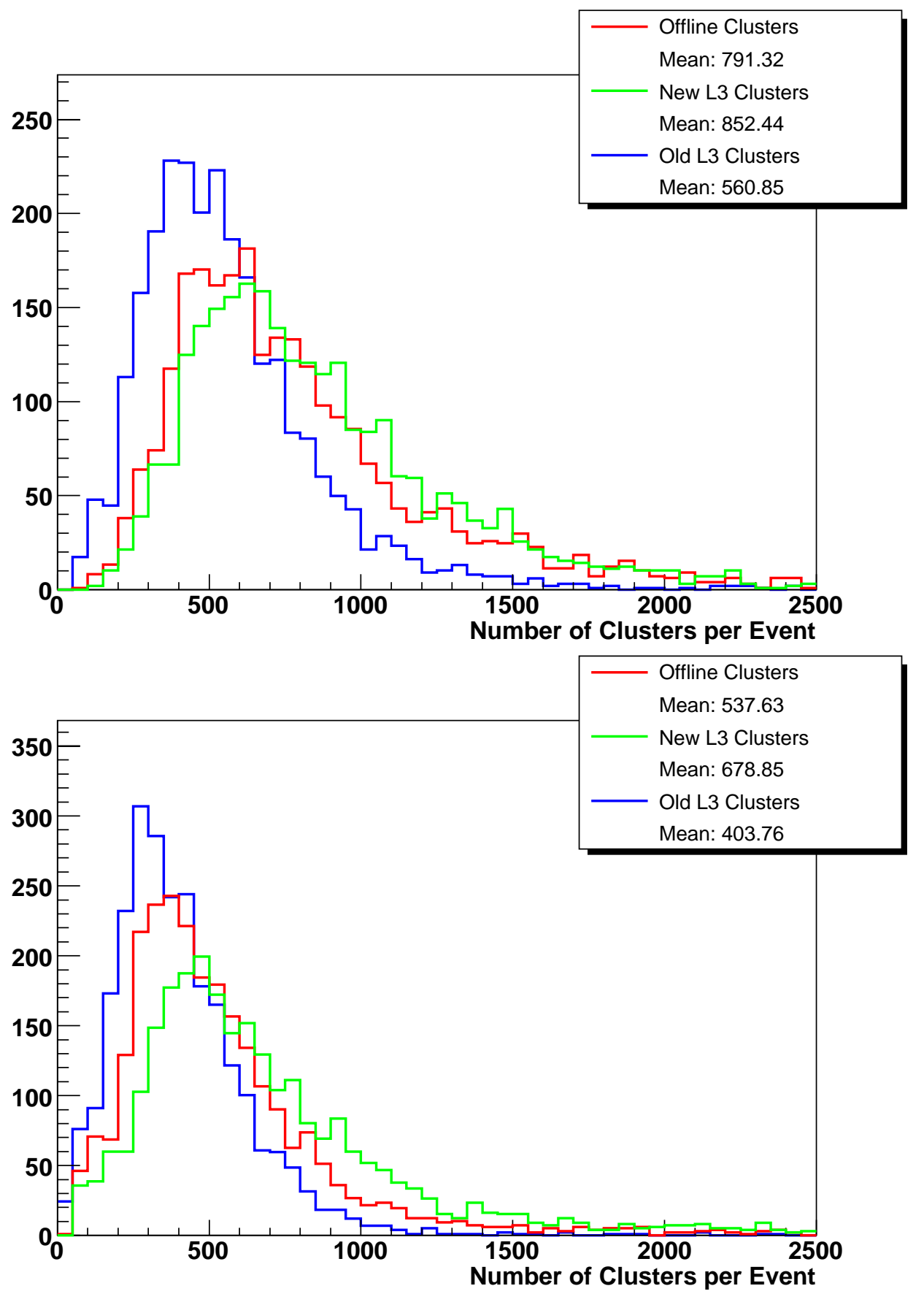

Figure 3.10: The number of axial clusters (top) and stereo clusters (bottom) per event found by the old L3 (blue), offline (red) and new L3 (green) SMT unpacking tools. 

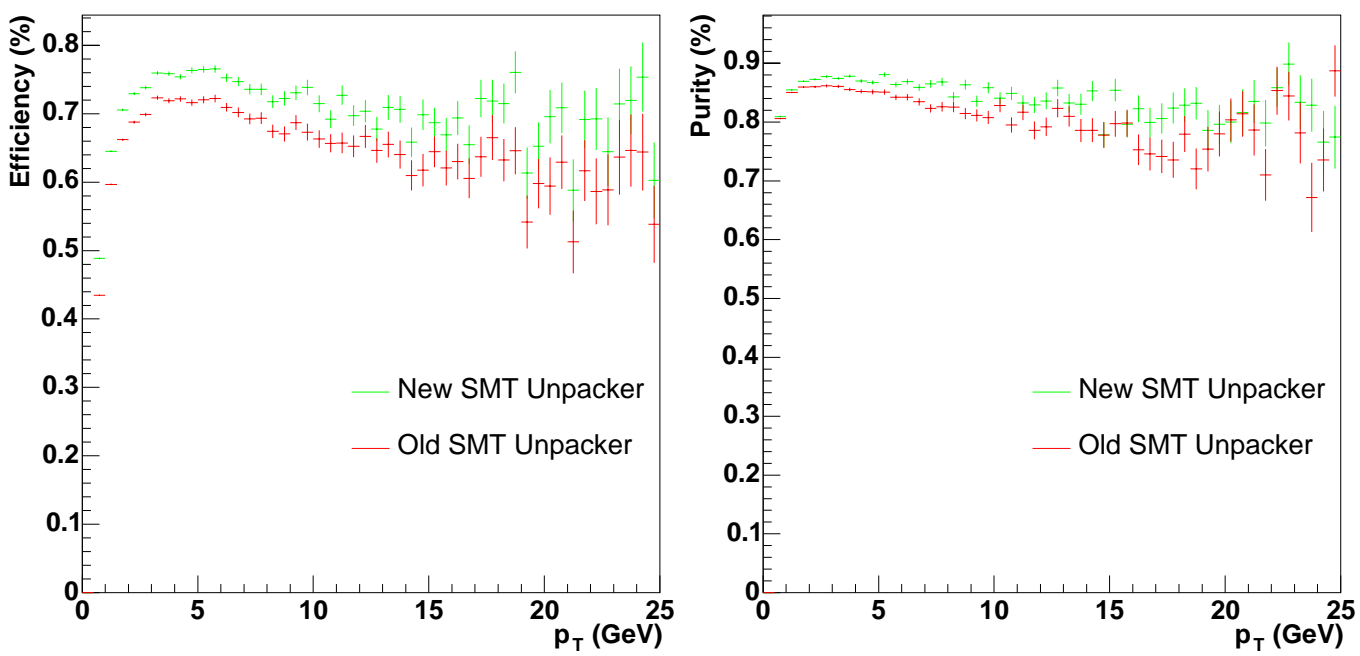

Figure 3.11: The track efficiency (left) and purity (right) found with the old L3 (red) and new L3 (green) SMT unpacking tools.

$\sim 70 \%$ in the number of axial and stereo clusters per track is achieved using the new L3 unpacking tool. As the new unpacker significantly increases the number of clusters associated with 'good' matched tracks, this adds confidence that the additional clusters are genuine.
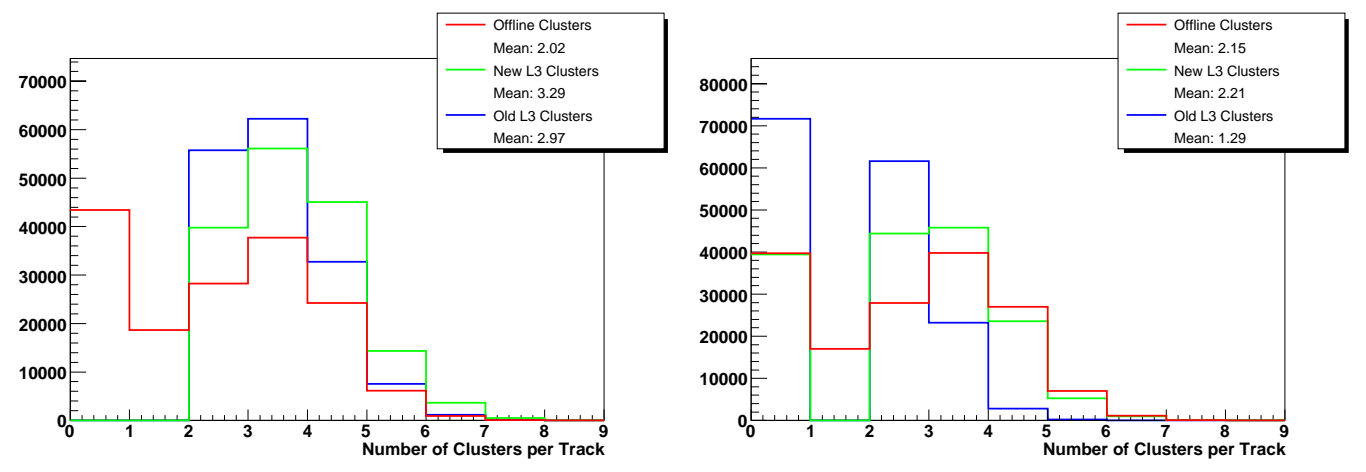

Figure 3.12: The number of axial (left) and stereo (right) SMT clusters associated with 'good' matched tracks (at least 8 CFT and 2 SMT clusters matched with an offline track) found using the old L3 (green) and new L3 (red) SMT unpacking tools.

\subsubsection{Tool Times}

Due to the limited time available at L3, the amount of time each tool takes to run needs to be carefully studied. Timing tests are carefully conducted on the same machine and under the same conditions to ensure consistency between different timing measurements - in this case a $2.4 \mathrm{GHz}$ Linux machine with no other jobs running. The timing results are shown in Table 3.3 and in Fig. 3.13. The new L3 
unpacking tool takes longer than the old tool due to the lower thresholds and the splitting algorithm. The increase in time taken is at a reasonable level and is an acceptable increase given the gain in the performance and quality of the tracking.

\begin{tabular}{|c|c|c|}
\hline \multirow{2}{*}{ Tool } & \multicolumn{2}{|c|}{ Timing $(\mathrm{ms})$} \\
\cline { 2 - 3 } & Old & New \\
\hline SMT Unpacker & 9.0 & 14.0 \\
Tracker & 10.2 & 9.6 \\
\hline
\end{tabular}

Table 3.3: The times measured for each component of the tracking before and after the upgrade of the L3 SMT unpacking tool. Errors on timing tests were estimated to be $\sim 10 \%$ from repeated measurements.
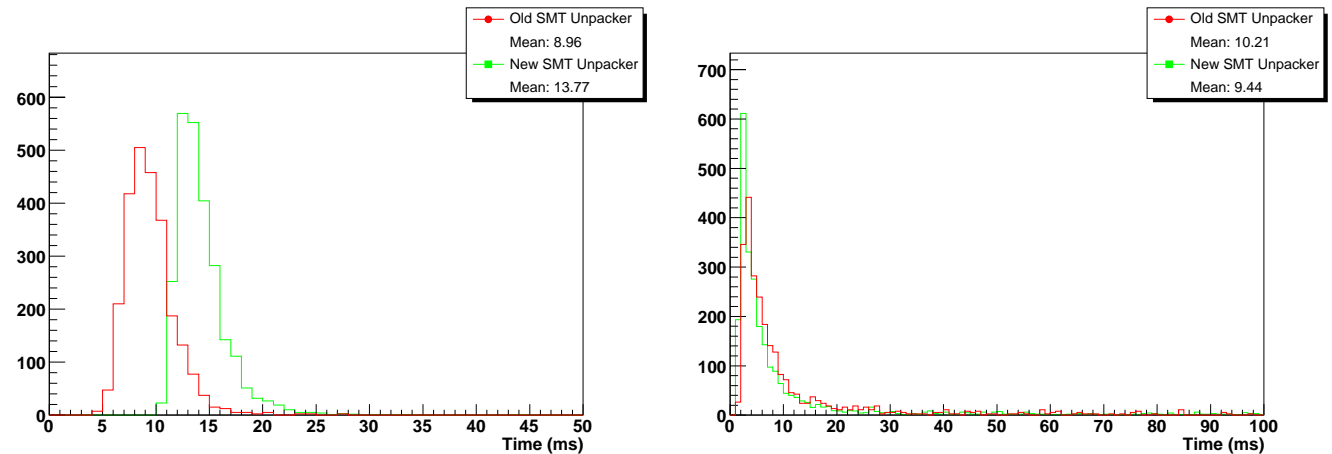

Figure 3.13: The timing distributions, in milliseconds, for the SMT unpacking tool (left) and the tracking tool (right) over 2500 events, when using the old (red) and new (green) SMT unpacking tools.

\subsubsection{Conclusion}

Studies into the L3 tracking efficiency and quality revealed problems with the L3 SMT clustering. Several improvements and fixes were implemented in the unpacking tool, namely: new pedestal thresholds, cluster errors, splitting and merging algorithms and 'hot' SVX chip protection. The improvements substantially increased the purity, efficiency and quality of the L3 tracks.

\subsection{SUSY Higgs Trigger Development}

\subsubsection{Introduction}

Triggers are arguably the first and most important (as they are irreversible) set of analysis cuts. A trigger which can efficiently select the signature of interest whilst 
minimising the acceptance of background events is therefore one of the most vital components of any analysis at a hadron collider. This section covers the development of the triggers for the multi-jet MSSM Higgs analysis. This multi-jet analysis is a search for the associated production of a Higgs with a $b$-quark, where the Higgs decays to $b \bar{b}$ resulting in a final state including 3 or more $b$-jets (see Section 5.1.1 for further details of this channel). The trigger is designed to select $h b \rightarrow b b b$ events whilst rejecting multi-jet QCD background.

This trigger study was conducted at the beginning of 2004, when two basic Higgs multi-jet triggers were running online as part of version 12 (v12) of the triggerlist. The v12 triggers were designed to cope with average luminosities of around $\sim 15 \times 10^{30} \mathrm{~cm}^{-2} \mathrm{~s}^{-1}$ and have a rate of $\sim 1.5 \mathrm{~Hz}$. Instantaneous luminosities averaging $\sim 60 \times 10^{30} \mathrm{~cm}^{-2} \mathrm{~s}^{-1}$ were expected by summer 2004. The triggers therefore needed upgrading to achieve a factor of 4 extra rejection to stay within their individual bandwidth allowance of $\sim 1.5 \mathrm{~Hz}$ averaged over the full range of expected luminosities; preferably whilst maintaining or improving the current signal efficiency. This study outlines the L3 trigger work the author carried out to improve the triggers to cope with the new high luminosity environment; the new triggers started running online in the summer of 2004 in version 13 (v13) of the triggerlist.

\subsubsection{Historical $h b$ Triggers}

Data have been collected with three different generations of the $h b$ multi-jet trigger, referred to as versions $\mathrm{v} 9 / 10, \mathrm{v} 11$ and $\mathrm{v} 12$. The triggers have become increasingly sophisticated as greater rejection has been required due to the increasing instantaneous luminosities.

The lowest level, L1, constructs calorimeter jet towers (CJT) which are characterised by the sum of the transverse energy $\left(E_{T}\right)$ in $0.2 \times 0.2 \Delta \phi$ vs $\Delta \eta$ cells in the calorimeter. The L1 trigger counts the number of CJTs with $E_{T}$ above a predefined threshold, from examining all the CJTs in an event. The L1 trigger then cuts on the number of CJTs fulfilling the predefined $E_{T}$ criteria.

The second level, L2, constructs simple jets from $5 \times 5$ CJT grids centred around seed CJTs which have $E_{T}$ above a predefined threshold; the jets stay centred on the seed tower and are not allowed to move. Depending on the number of shared towers (also set by a predefined threshold), overlapping L2 jets or either classified as separate jets or only the highest $E_{T}$ tower is kept. The L2 triggers cut on the number of jets with $E_{T}$ above a threshold and on the the total scalar sum of the $E_{T}$ of the jets, $H_{T}$. 
The highest trigger level, L3, constructs jets using a simple cone algorithm using a radius of either 0.5 (SC5) or 0.7 (SC7). Triggers cut on the $E_{T}$ and $H_{T}$ of the jets. A terminology is adapted for the L3 jets of $\mathrm{JT}(n, c)$ where $n$ is the number of jets and $c$ is the energy threshold.

In v11 and v12 the requirement of a PV with $|z|<35 \mathrm{~cm}$ was added at L3. This cut ensures only events which are within the fiducial range of the SMT are selected, and provides additional background rejection for a negligible loss in efficiency.

In v12 of the triggerlist a 'Muon' branch was introduced to complement the 'Jet' branch. The 'Muon' branch has the additional requirement of a L2 muon, taking advantage of the fact that a $b$-jet has a $10 \%$ probability of decaying into a high $\mathrm{p}_{T}$ muon whereas $<1 \%$ of light jets will [51]. The extra rejection gained from a muon requirement can then be used to loosen the L3 jet cuts. A terminology is adapted for the L3 Muons of $\mathrm{MU}(n, q, c)$ where $n$ is the number, $q$ is the quality (loose, medium or tight) and $c$ is the $\mathrm{p}_{T}$ cut on the muon.

The three generations of the $h b$ multi-jet trigger are outlined below:

Triggerlist - v9/10

Name - 3JT15

L1 - 4 CJT with $E_{T}>5 \mathrm{GeV}$

L2 - 3 L2 Jets with $E_{T}>8 \mathrm{GeV}$ and $H_{T}>50 \mathrm{GeV}$ constructed using jets with $E_{T}>5 \mathrm{GeV}$

L3 - 3 SC7 L3 Jets with $E_{T}>15 \mathrm{GeV}$ and $|\eta|<3$

Triggerlist - v11

Name - 3JT15_PV 'Jet' Branch

L1 - 3 CJT with $E_{T}>5 \mathrm{GeV}$

L2 - 3 L2 Jets with $E_{T}>8 \mathrm{GeV}$ and $H_{T}>50 \mathrm{GeV}$ constructed using jets with $E_{T}>5 \mathrm{GeV}$

L3 - 3 SC7 L3 Jets with $E_{T}>15 \mathrm{GeV}, 2$ with $E_{T}>25 \mathrm{GeV}$ and all with $|\eta|<3$, and a PV with $|z|<35 \mathrm{~cm}$

Name - 3JT15_L2L0_PV 'Muon' Branch

L1 - 3 CJT with $E_{T}>5 \mathrm{GeV}$ 
L2 - 3 L2 Jets with $E_{T}>8 \mathrm{GeV}, H_{T}>50 \mathrm{GeV}$ constructed using jets with $E_{T}>5 \mathrm{GeV}$ and a Loose L2 Muon

L3 - 3 SC7 L3 Jets with $E_{T}>15 \mathrm{GeV}$ and $|\eta|<3$, and a PV with $|z|<35 \mathrm{~cm}$

Triggerlist - v12 'Jet' Branch

Name - 3JT15_2J25_PV

L1 - 3 CJT with $E_{T}>5 \mathrm{GeV}$

L2 - 3 L2 Jets with $E_{T}>8 \mathrm{GeV}$ and $H_{T}>50 \mathrm{GeV}$ constructed using jets with $E_{T}>5 \mathrm{GeV}$

L3 - 3 SC5 L3 Jets with $E_{T}>15 \mathrm{GeV}, 2$ with $E_{T}>25 \mathrm{GeV}$ and all with $|\eta|<3$, and a PV with $|z|<35 \mathrm{~cm}$

Name - 3JT15_PV 'Muon' Branch

L1 - 3 CJT with $E_{T}>5 \mathrm{GeV}$

L2 - 3 L2 Jets with $E_{T}>8 \mathrm{GeV}, H_{T}>50 \mathrm{GeV}$ constructed using jets with $E_{T}>5 \mathrm{GeV}$ and a Loose L2 Muon with no $\mathrm{p}_{T}$ cut

L3 - 3 SC5 L3 Jets with $E_{T}>15 \mathrm{GeV}$ and $|\eta|<3$, and a PV with $|z|<35 \mathrm{~cm}$

\subsubsection{Trigger Development}

Trigger development is a delicate balance between several considerations: the signal efficiency, the rate and available bandwidth for the trigger, the evolution of the instantaneous luminosity and any resulting pre-scales which may be applied to the triggers, and the overlap between different triggers.

\subsubsection{Level 1 and Level 2 Trigger Development}

The total number of L1 trigger bits and the total bandwidth available at L1 is limited. L1 trigger bits are therefore shared between physics channels with similar topologies. Each L1 trigger term is agreed upon by the various analyses whose triggers will branch from the L1 trigger term. The final decision is taken by the Trigger Board who make the final decision on all trigger related matters [68]. Their considerations are based upon the total bandwidth available, the evolution of the instantaneous luminosities and the Collaboration's physics goals and priorities. 
The triggers used in the $h b$ analysis share L1 trigger terms with other multi-jet topologies, such as $Z H \rightarrow \nu \nu b \bar{b}$ and $t \bar{t}$ all of which have at least 2 jets and no charged leptons in the final state. For the v13 triggerlist a new L1 trigger term for the multi-jet events of $2 \mathrm{CJT}$ with $E_{T}>5 \mathrm{GeV}$ and $|\eta|<2.6$, and 3 CJT with $E_{T}<4 \mathrm{GeV}$ and $|\eta|<3.2$ was introduced. The new L1 term increases the signal efficiencies for all the channels whilst remaining within the L1 bandwidth restraint of $\sim 5 \mathrm{kHz}$. Due to the looser L1 requirements the L2 trigger requirements needed redesigning to remain within the L2 constraints. Two new L2 trigger terms were developed after a detailed optimisation [69]. The optimal 'Jet' branch trigger cuts were selected as $3 \mathrm{~L} 2$ jets with $E_{T}>6 \mathrm{GeV}$ and a $H_{T}>60 \mathrm{GeV}$ cut constructed from jets with $E_{T}>6 \mathrm{GeV}$. The optimal 'Muon' branch trigger cuts were selected as $H_{T}>60 \mathrm{GeV}$ with a Loose $\mathrm{L} 2$ muon with no $\mathrm{p}_{T}$ cut.

\subsubsection{Level 3 Trigger Development}

Signal Efficiency: The trigger efficiency is defined as:

$$
\text { Efficiency }=\frac{\text { Number of L1L2L3 Triggered Events }}{\text { Number of L1L2 Triggered Events }},
$$

where L1L2 refers to events which have passed the L1 and L2 trigger conditions and L1L2L3 refers to events which have passed the L1, L2 and L3 trigger conditions. The signal efficiency is measured relative to the number of events with three offline jets with $E_{T}>8 \mathrm{GeV}$, which represents the minimum offline analysis criteria. Trigger efficiencies are measured relative to the loosest possible analysis criteria, as opposed to using the tighter final analysis cuts, to minimise any bias during development.

The efficiencies were measured using $24000 h b \rightarrow b b b$ Pythia [70] MC events with a Higgs mass, $m_{h}=100 \mathrm{GeV}$, which is the lowest $m_{h}$ considered in the $h b$ analysis. The mass of the Higgs particle is unknown, and so a Higgs trigger has to be designed to work effectively for a range of Higgs masses. It is preferable to optimise a Higgs trigger for the lowest potential Higgs mass, as on average these events have the softest spectrum, and correspondingly need the loosest cuts. A trigger optimised for the lowest Higgs mass will work more effectively for higher Higgs mass points as they have a harder spectrum [71].

Background Rejection: The background rejection efficiency is defined as:

$$
\text { Rejection Efficiency }=1-\frac{\text { Number of L1L2L3 Triggered Events }}{\text { Number of L1L2 Triggered Events }} \text {. }
$$


The rejection was measured on data, as MC is not an entirely realistic representation of the background data, assuming that there is a negligible contribution from any actual signal. 'Special Runs', taken using the finalised L1L2 trigger list with no L3 terms, were used to measure the final trigger rate. The trigger rate was measured at the instantaneous luminosity of the 'Special Run', and was given by:

$$
\text { Rate }=\text { L1L2 Rate } \times \frac{\text { Number of L1L2L3 events }}{\text { Number of L1L2 events }}
$$

where the L1L2 Rate is the trigger rate for the L1L2 trigger terms measured in the 'Special Run'. The rates and background rejection are measured on run 191322 which was taken at an instantaneous luminosity of $60 \times 10^{30} \mathrm{~cm}^{-2} \mathrm{~s}^{-1}$.

Trigger Development Strategy: The desired extra rejection was achieved in both trigger branches by using new tools which provided a more powerful handle on background rejection; any extra rejection above the desired level was used to loosen the other cuts.

As the extra trigger requirements lead to a loss of efficiency, the second part of the strategy was to consider the joint efficiency of the 'Jet' and 'Muon' branches to ensure that the overall efficiency was maintained or even improved. The v12 triggers had a $100 \%$ overlap in the L3 trigger terms, leading to a large replication in triggered signal events. Making the v13 triggers sufficiently different reduced this overlap, and allowed a higher joint efficiency, even though each trigger had a lower individual efficiency compared to the v12 triggers.

The 'Jet' branch gained extra rejection by requiring a $b$-jet using the L3 IP $b$-tagging tool. The v12 trigger had no $b$-jet requirement and so significant gains were possible as the signal consists of at least three $b$-jets and the majority of the background consists of light-jets. The 'Muon' branch trigger, which already required a L2 muon, used the additional requirement of a L3 muon to gain the extra rejection.

The efficiency and rejection given by various L3 variables were examined in order of discriminating power. For the 'Jet' branch, the L3 IP b-tag cut was examined first, followed by the $E_{T}$ cut on the $1^{\text {st }}, 2^{\text {nd }}$ and $3^{\text {rd }}$ leading jets. For the 'Muon' branch the L3 Muon $\mathrm{p}_{T}$ cut was optimised first, followed by the $E_{T}$ cut on the $1^{\text {st }}, 2^{\text {nd }}$ and $3^{\text {rd }}$ leading jets. The final trigger cuts were selected by scanning all the possible different cut combinations and selecting those which produced the necessary rejection with the highest joint trigger efficiency. 


\subsubsection{3 'Jet' Branch}

The signal and background rejection efficiencies of the L3 IP $b$-tagging tool relative to events which have passed the L1L2 trigger conditions are shown in Fig. 3.14. The L3 IP b-tagging tool is a very powerful tool and a large background rejection is possible for a minimal loss in efficiency. A L3 IP $b$-tag $<0.05(\operatorname{IP}(0.05))$ cuts $90 \%$ of the background events whilst maintaining a signal efficiency of $>80 \%$.
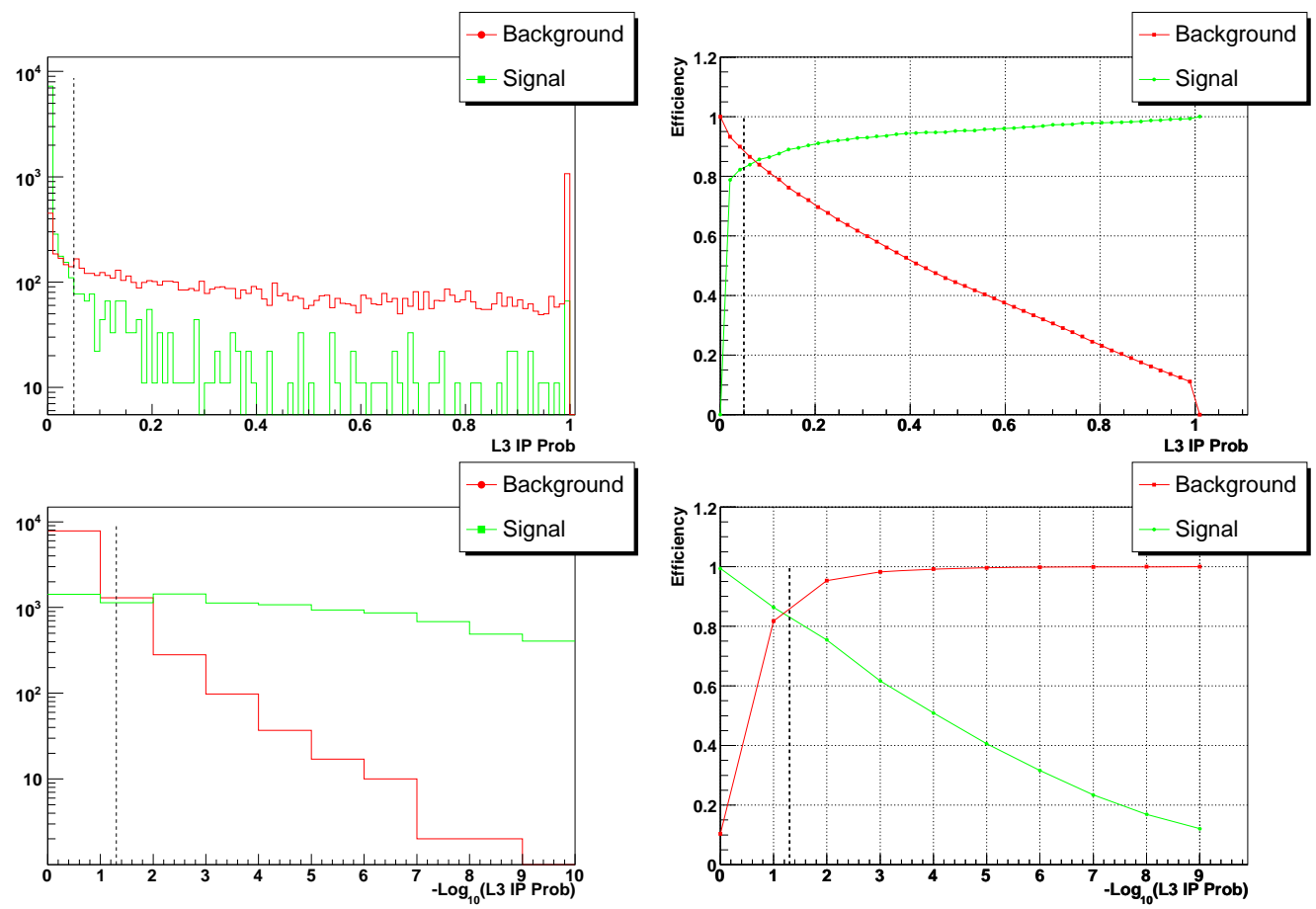

Figure 3.14: Left: The L3 IP $b$-tag distribution for the $h b$ signal sample (green) and the background (red). Right: The signal (green circles) and background (red squares) efficiencies as a function of the L3 IP $b$-tag cut. The black dotted lines are at a cut value of 0.05 .

The signal and background efficiencies of the $1^{\text {st }}, 2^{\text {nd }}$ and $3^{\text {rd }}$ leading jets relative to the number of events which have passed the L1L2 and L3 IP(0.05) cuts are shown in Fig. 3.15. The signal jets have a harder $E_{T}$ spectrum than the background jets. This allows background events to be rejected by cutting on the jet's $E_{T}$ without hurting the signal efficiency. However not much can be gained by altering the current jet cuts $(25,25$ and $15 \mathrm{GeV}$ respectively).

\subsubsection{4 'Muon' Branch}

The signal and background rejection efficiencies for various L3 muon $\mathrm{p}_{T}$ cuts, relative to the number of events which have passed the L1L2 trigger conditions, are shown in Fig. 3.16. By requiring a Loose L3 muon, a background rejection efficiency of 

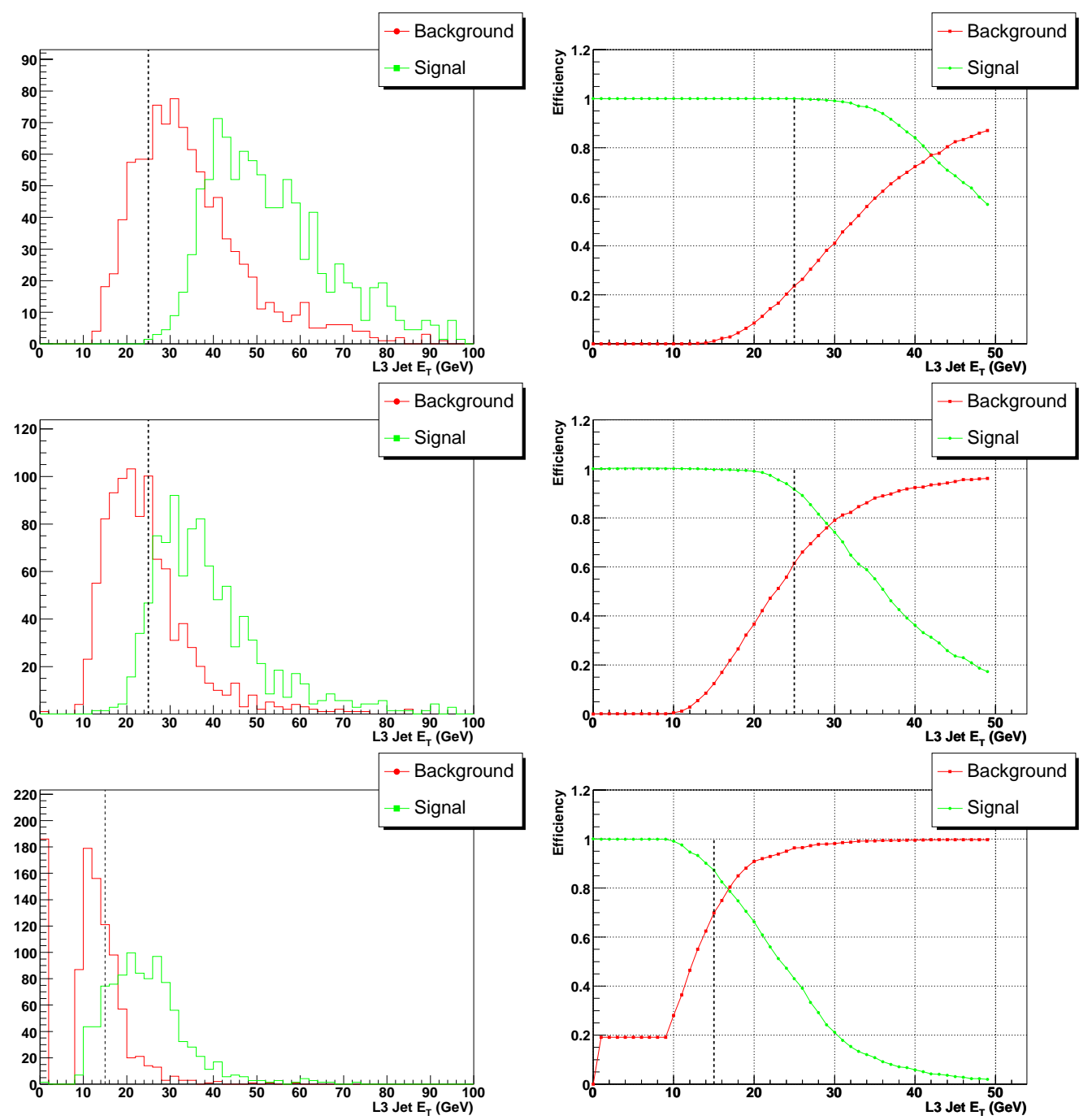

Figure 3.15: Left: The $1^{\text {st }}$ (top), $2^{\text {nd }}$ (middle) and $3^{\text {rd }}$ (bottom) leading jet $E_{T}$ for the $h b$ signal sample (green) and the background (red). Right: The trigger efficiencies for signal (green circles) and background (red squares) as a function of the jet $E_{T}$ cut on the $1^{\text {st }}$ (top), $2^{\text {nd }}$ (middle) and $3^{\text {rd }}$ (bottom) leading jets after the L1L2 cuts and a L3 $b$-tag IP cut $<0.05$. The black dotted lines are at the v12 cut values. 
$\sim 90 \%$ is achieved at the expense of $\sim 45 \%$ drop in the signal efficiency. The large rejection achieved by the L3 muon can be used to loosen the L3 jet cuts to recover some of the lost efficiency. A 'Loose' muon is one which has passed through the iron toroid, and so will therefore most likely have $p_{T}>3 \mathrm{GeV}$, which is the optimal threshold.
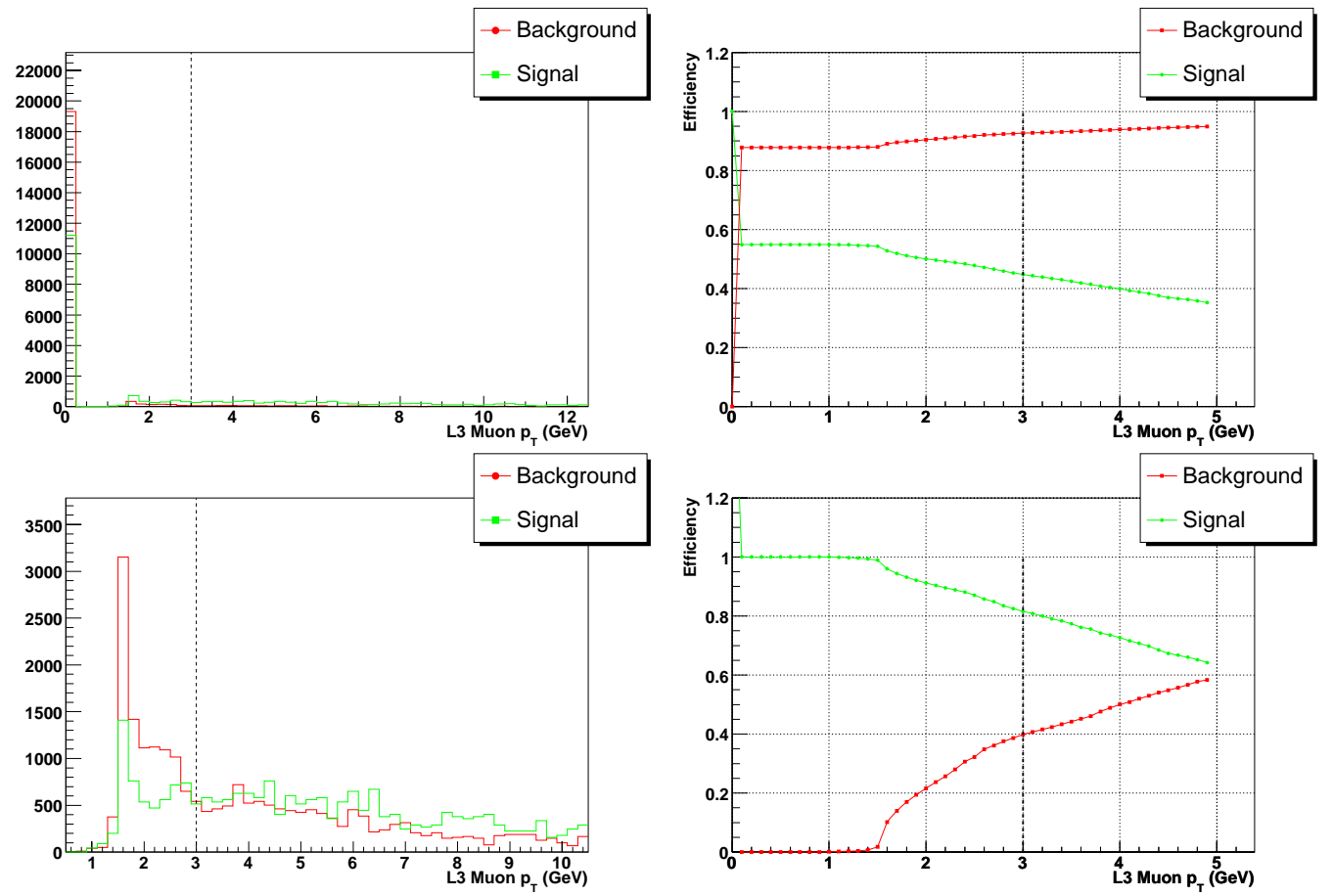

Figure 3.16: Left: The L3 Loose muon $\mathrm{p}_{T}$ distribution in the $h b$ signal sample (green) and the background (red), before (top) and after (bottom) requiring a Loose L3 muon. Right: The signal (green circles) and background (red squares) efficiencies as a function of the L3 muon $\mathrm{p}_{T}$ cut before (top) and after (bottom) requiring a Loose L3 muon. The black dotted lines are at a cut value of $3.0 \mathrm{GeV}$.

The signal and background rejection efficiencies for various $E_{T}$ cuts on the $1^{\text {st }}$, $2^{\text {nd }}$ and $3^{r d}$ leading L3 jets, relative to the number of events which have passed the L1L2 and Loose L3 Muon $p_{T}>3 \mathrm{GeV}$ cuts, are shown in Fig. 3.17. Signal jets have a harder $E_{T}$ spectrum than the background jets. This allows background to be rejected by cutting on the jet's $E_{T}$, whilst not hurting the signal efficiency. The current cut of $15 \mathrm{GeV}$ on each of the 3 leading L3 jets does not cut hard enough on the leading jet and cuts too hard on the $3^{\text {rd }}$ leading jet. Rejection gained by tightening the cut on the first leading jet can therefore be used to lower the cut on the $3^{\text {rd }}$ leading jet, which is currently rejecting approximately a third of the signal. This will increase the signal efficiency whilst having a negligible effect on the background rate. 

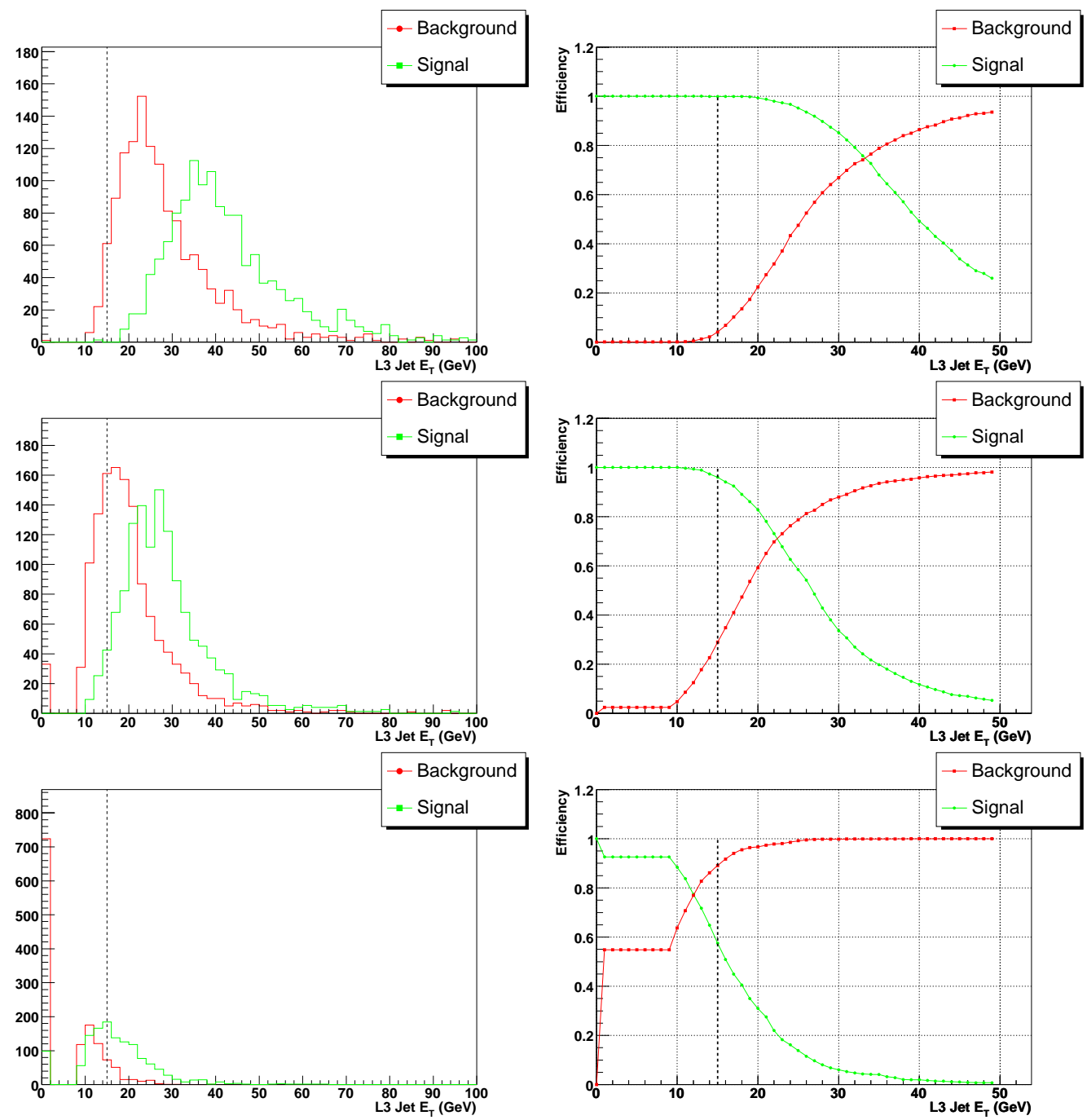

Figure 3.17: Left: The $1^{\text {st }}$ (top), $2^{\text {nd }}$ (middle) and $3^{\text {rd }}$ (bottom) leading jet $E_{T}$ distributions for the $h b$ signal sample (green) and the background (red). Right: The trigger efficiencies for signal (green circles) and background (red squares) as a function of the jet $E_{T}$ cut on the $1^{s t}$ (top), $2^{\text {nd }}$ (middle) and $3^{\text {rd }}$ (bottom) leading jets, after the L1L2 cuts and a Loose L3 Muon with $p_{T}>3 \mathrm{GeV}$. The black dotted lines are at the v12 cut values. 


\subsubsection{L3 Impact Parameter $b$-tagging Tool Data Tests}

The L3 IP b-tagging tool had not been used in any triggers prior to these studies and it was important that the tool was thoroughly tested on data. Various tests were carried out on the L3 IP $b$-tagging tool to ensure that it functioned as expected on data events. These tests were all carried out using a 'special run', run number 179621 which was recorded at a instantaneous luminosity of $13 \times 10^{30} \mathrm{~cm}^{-2} \mathrm{~s}^{-1}$, which consists of high $\mathrm{p}_{T}$ muon triggered data and is therefore $b$-enhanced. The IP significance of the L3 tracks is shown in Fig. 3.18 for all jets (left) and for jets from events with at least one offline JLIP Prob $<0.003$ tag (right). The fit to the negative IP significance is reflected into the positive IP significance distribution to highlight the large excess of tracks with positive IP significance. A clear excess of tracks with large positive IP significance is seen for those events with an offline JLIP $b$-tag. This large excess demonstrates that the L3 tool is correctly identifying the displaced tracks from $b$-jets.
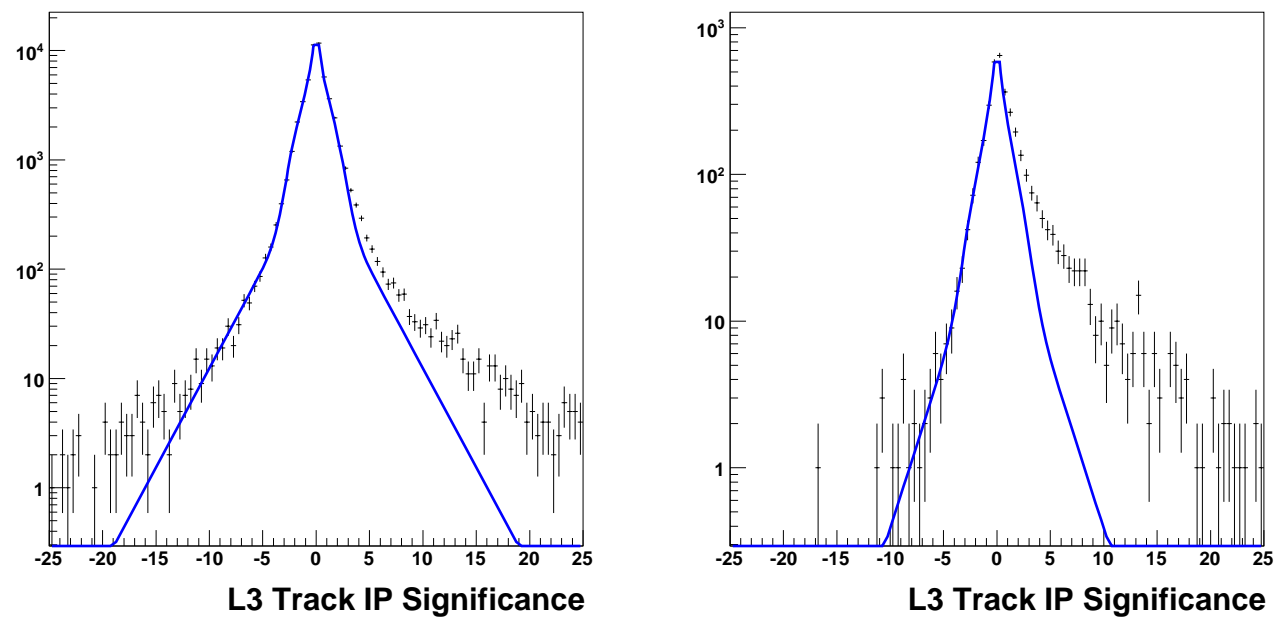

Figure 3.18: The L3 track IP (IP) significancies for all events (left) and for those with at least one offline JLIP tag (right) in a $b$-enriched 'special run'. The fit is a double exponential and a gaussian.

The L3 IP probability for jets which pass an offline JLIP probability cut of 0.003 , 0.01 and 0.02 are shown in Fig. 3.19. The L3 IP b-tagging tool either correctly tags the jets (peak at 0) or does not have the necessary tracks (peak at 1). A L3 IP cut of 0.05 has an efficiency of $\sim 60 \%$ for the tightest JLIP cut. The efficiency to tag an offline JLIP tagged jet is shown in Fig. 3.20 (left) as a function of the L3 IP jet probability. The single jet efficiencies can then be used to calculate the probability to tag an event with three offline JLIP jets using the following equation: 


$$
P_{J L I P}^{3}=1-\left(1-P_{J L I P}^{1}\right)^{3}
$$

where $P_{J L I P}^{1}$ is the probability to tag an offline tagged JLIP jet and $P_{J L I P}^{3}$ is the probability to tag an event with three offline tagged JLIP jets. The efficiency to tag an event with three offline JLIP tags is shown in Fig. 3.20 (right). For a cut of $\operatorname{IP}(0.05)$ the probability to tag an event with three $\operatorname{JLIP}(0.003)$ jets is estimated to be $>90 \%$.

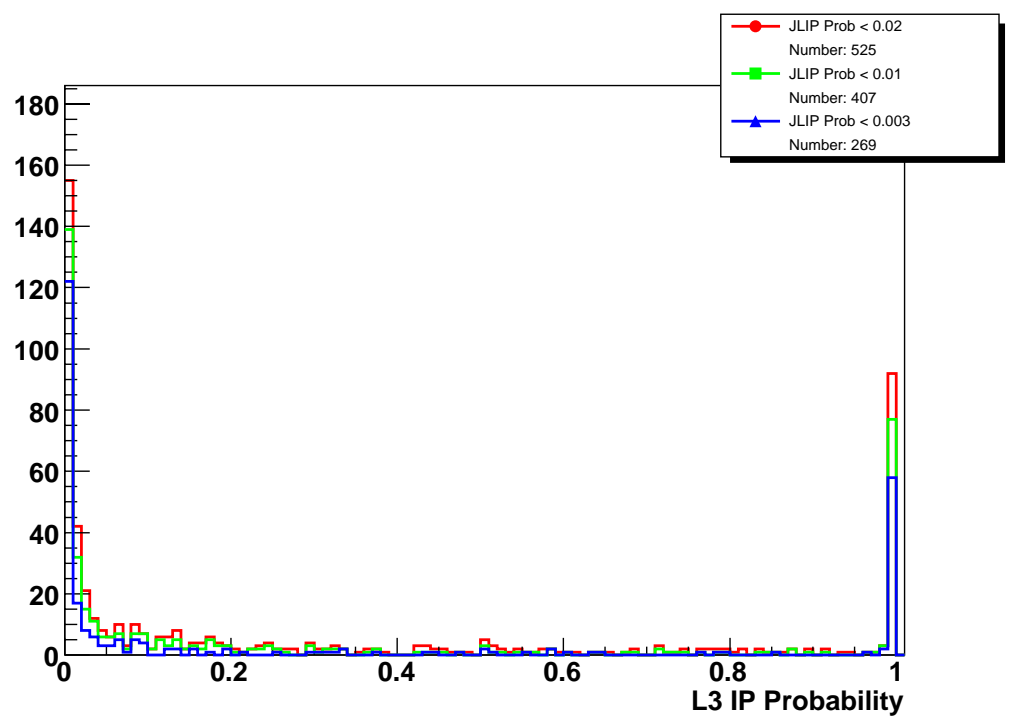

Figure 3.19: The L3 IP $b$-tagging tool output for jets which pass a JLIP probability cut of 0.003 (blue line), 0.01 (green line) and 0.02 (red line). The spike at one corresponds to jets with too few tracks at L3 with which to calculate a $b$-tag.
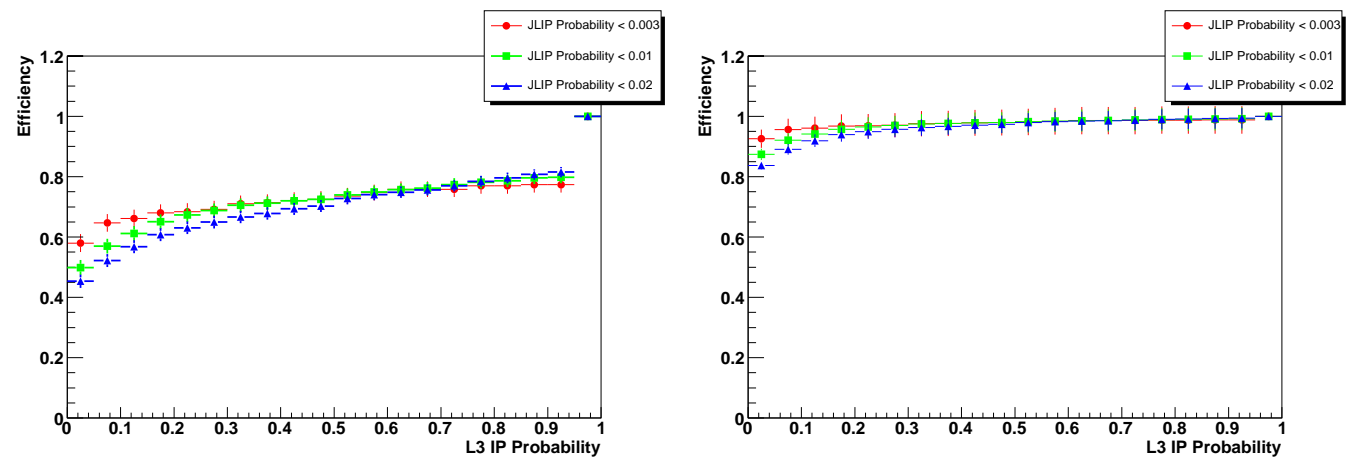

Figure 3.20: Left: The L3 IP $b$-tagging tool's efficiency to tag jets which have a JLIP cut of 0.003 (blue line), 0.01 (green line) and 0.02 (red line). Right: The L3 IP $b$-tagging tool's efficiency to tag events which have three jets with JLIP cuts of 0.003 (blue line), 0.01 (green line) and 0.02 (red line). 


\subsubsection{Final Trigger Selection}

The final L3 trigger terms were selected by scanning all the possible cut combinations, and selecting the triggers which gave the highest joint efficiency for individual rates of $1.5 \mathrm{~Hz}$. The final trigger selections for the 'Jet' and 'muon' triggers are shown below:

Triggerlist - v13 'Jet' Branch

Name - JT2_3JT15L_IP_VX

L1 - 2 CJT with $E_{T}>5 \mathrm{GeV}$ and 3 CJT with $E_{T}>4 \mathrm{GeV}$ with $|\eta|<2.4$

L2 - 3 L2 Jets with $E_{T}>6 \mathrm{GeV}$ and $H_{T}>70 \mathrm{GeV}$ constructed with jets with $E_{T}>6 \mathrm{GeV}$

L3 - L3 IP $b$-tagging Prob $<0.05,3$ SC5 L3 Jets with $E_{T}>15 \mathrm{GeV}$ and $|\eta|<3$, 2 with $E_{T}>25 \mathrm{GeV}$ and $|\eta|<3$, and a PV with $|z|<35 \mathrm{~cm}$

Triggerlist - v13 'Muon' Branch

Name - JT3_3JT10L_LM3_V

L1 - 2 CJT with $E_{T}>5 \mathrm{GeV}$ and $3 \mathrm{CJT}$ with $E_{T}>4 \mathrm{GeV}$ with $|\eta|<2.4$

L2 - $H_{T}>30 \mathrm{GeV}$ constructed with jets with $E_{T}>6 \mathrm{GeV}$ and a Loose L2 Muon

L3 - L3 Muon with $p_{T}>3 \mathrm{GeV}, 3$ SC5 L3 Jets with $E_{T}>15 \mathrm{GeV}$ and $|\eta|<3$, 1 with $E_{T}>25 \mathrm{GeV}$ and $|\eta|<3$, and a PV with $|z|<35 \mathrm{~cm}$

The signal efficiency versus rate for the 'Jet' branch of the trigger is shown in Fig. 3.21 as the L3 conditions are varied. Each line represents a cut on a L3 tool after previous cuts have been applied and the points along the line represent the different cut values for that tool. The majority of the rejection at L3 comes from the L3 IP $b$-tagging tool which reduces the rate from $113 \mathrm{~Hz}$ to $11 \mathrm{~Hz}$, with a relative loss in signal efficiency of only $10 \%$. The cut on the $1^{\text {st }}$ leading jet (red line) decreases the rate to about $8 \mathrm{~Hz}$ with minimal loss of efficiency. The cut on the $2^{\text {nd }}$ leading jet (green line) results in a 10\% relative loss in efficiency whilst decreasing the rate to $4 \mathrm{~Hz}$. The cut on the $3^{r d}$ leading jet (blue line) also results in a relative loss of $10 \%$ in efficiency whilst decreasing the rate from 4 to $2 \mathrm{~Hz}$.

The signal efficiency versus rate for the 'muon' branch of the trigger is shown in Fig. 3.22 as the L3 conditions are varied. Each line represents a cut on a L3 tool 


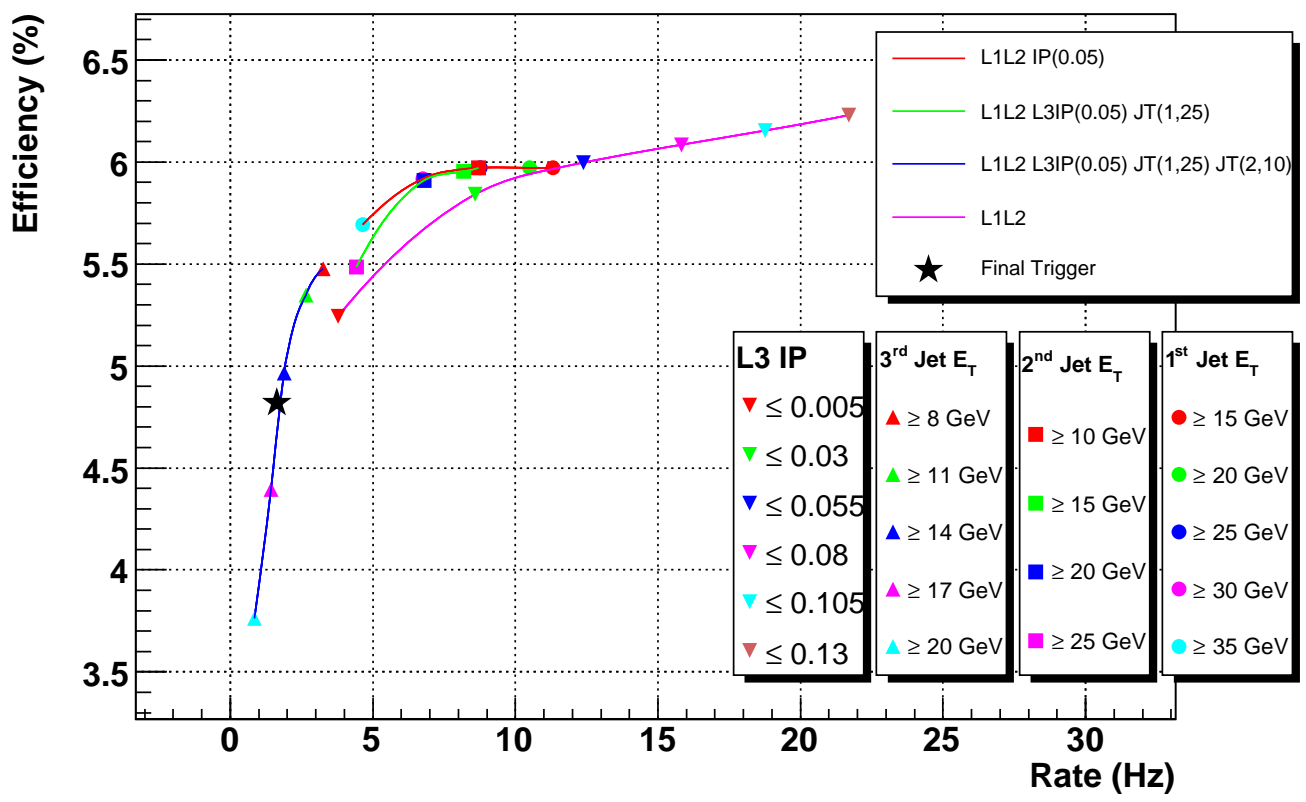

Figure 3.21: The L1L2L3 signal efficiency of various trigger cuts versus the rate to tape at a luminosity of $60 \times 10^{30} \mathrm{~cm}^{-2} \mathrm{~s}^{-1}$. Each line represents a cut on a L3 tool and the points along the line represent the different cut values. The black star is the final trigger.

after the previous cuts have been applied, and each point represents a different cut values for that tool. The majority of the rejection for the 'muon' branch comes from the L3 Muon requirement (pink line) which reduces the rate from $15 \mathrm{~Hz}$ to $0.9 \mathrm{~Hz}$, with the efficiency decreasing from $14 \%$ to $7.4 \%$. The cut on the $1^{\text {st }}$ leading jet (red line) decreases the rate to about $3 \mathrm{~Hz}$ with minimal loss of efficiency. The cut on the $2^{\text {nd }}$ leading jet (green line) has a minimal effect on either the efficiency or rate. The cut on the $3^{\text {rd }}$ leading jet (blue line and triangle points) cuts the rate from 3 $\mathrm{Hz}$ to $1 \mathrm{~Hz}$ and the efficiency from $7.2 \%$ to $6.2 \%$.

\subsubsection{Trigger Performance}

The efficiencies of the triggers measured on the $h b \mathrm{MC}$ sample are shown in Table 3.4. The new triggers have a joint efficiency which is $\sim 40 \%$ higher than the v12 triggers whilst obtaining the necessary additional factor of 4 rejection for both triggers.

\subsubsection{Online and Future Performance}

The rate of the triggers running online for various instantaneous luminosities are shown in Table 3.5, with both triggers having performed within their design goals. 


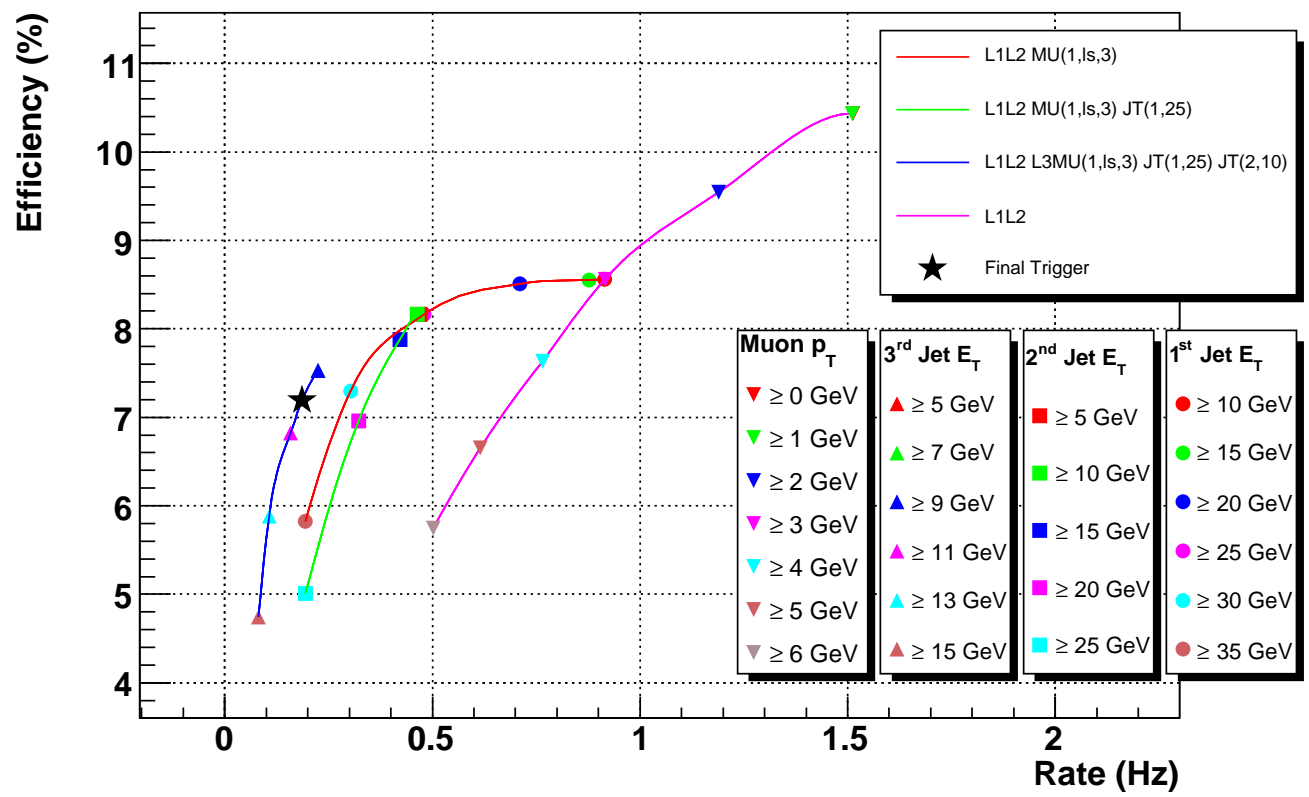

Figure 3.22: The L1L2L3 signal efficiency of various trigger cuts versus the rate to tape at a luminosity of $60 \times 10^{30} \mathrm{~cm}^{-2} \mathrm{~s}^{-1}$. Each line represents a cut on a L3 tool and the points along the line represent the different cut values. The black star is the final trigger.

\begin{tabular}{|c|c|c|c|c|c|}
\hline \multirow{2}{*}{$\begin{array}{c}\text { Trigger } \\
\text { List }\end{array}$} & \multirow{2}{*}{ Trigger } & \multicolumn{4}{|c|}{ Efficiency (\%) } \\
\cline { 3 - 6 } & & L1 & L2 & L3 & L1L2L3 \\
\hline \multirow{3}{*}{ v12 } & 3JT15_PVZ & $44.4 \pm 2.6$ & $6.3 \pm 0.4$ & $35.0 \pm 2.0$ & $4.3 \pm 0.3$ \\
& 3J15_2J25_PVZ & $44.4 \pm 2.6$ & $12.5 \pm 0.8$ & $21.4 \pm 1.3$ & $7.1 \pm 0.5$ \\
\cline { 2 - 6 } & OR/Both & $44.4 \pm 2.6$ & $12.5 \pm 0.8$ & $35.0 \pm 2.0$ & $7.8 \pm 0.5$ \\
\hline \multirow{4}{*}{ v13 } & JT3_3JT10L_LM3_V & $54.1 \pm 3.1$ & $26.1 \pm 1.5$ & $13.2 \pm 0.8$ & $7.2 \pm 0.5$ \\
& JT2_3JT15L_IP_VX & $54.1 \pm 3.1$ & $7.6 \pm 0.5$ & $18.1 \pm 1.1$ & $4.8 \pm 0.3$ \\
\cline { 2 - 6 } & OR/Both & $54.1 \pm 3.1$ & $29.8 \pm 1.7$ & $27.3 \pm 1.6$ & $11.0 \pm 0.7$ \\
\hline
\end{tabular}

Table 3.4: Efficiency (\%) of the v12 and v13 triggers on events which have a minimum of 3 offline jets with $E_{T}>8 \mathrm{GeV}$, measured on the $h b\left(m_{h}=100 \mathrm{GeV}\right) \mathrm{MC}$ sample. The OR of the triggers measures the joint efficiency of the two triggers. The errors contain both a statistical and systematic $(\sim 5.5 \%[71])$ component. 


\begin{tabular}{|c|c|c|c|c|}
\hline \multirow{2}{*}{ Trigger } & Instantaneous & \multicolumn{3}{|c|}{ Rate $(\mathrm{Hz})$} \\
\cline { 3 - 5 } & Luminosity $\left(\mathrm{cm}^{-2} \mathrm{~s}^{-1}\right)$ & L1 & L2 & L3 \\
\hline \multirow{3}{*}{ JT2_3JT15L_IP_VX } & $40 \times 10^{30}$ & 167 & 47.2 & 0.95 \\
& $60 \times 10^{30}$ & 206 & 65.7 & 1.26 \\
\cline { 3 - 5 } & $80 \times 10^{30}$ & 327 & 93.1 & 1.91 \\
\hline \multirow{3}{*}{ JT3_3JT10L_LM3_V } & Average & 233 & 68.7 & 1.37 \\
& $40 \times 10^{30}$ & 167 & 25.4 & 0.72 \\
\cline { 2 - 5 } & $60 \times 10^{30}$ & 206 & 34.0 & 1.06 \\
\cline { 2 - 5 } & $80 \times 10^{30}$ & 327 & 56.0 & 1.75 \\
\hline
\end{tabular}

Table 3.5: The rates for the two v13 $h b$ triggers for an average instantaneous luminosity of $40 \times 10^{30} \mathrm{~cm}^{-2} \mathrm{~s}^{-1}$ measured on run 207292, $60 \times 10^{30} \mathrm{~cm}^{-2} \mathrm{~s}^{-1}$ measured on run 206944 and $80 \times 10^{30} \mathrm{~cm}^{-2} \mathrm{~s}^{-1}$ measured on run 206692. The rates for trigger JT3_3JT10L_LM3_V in run 206944 are corrected to account for a pre-scale of 5 . The average rates over the range of instantaneous luminosities are within the design goals.

The L3 'Jet' term remained unchanged through the v13 (June 2004 to July 2005) and the v14 (July 2005 to June 2006) triggerlists collecting $\sim 600 \mathrm{pb}^{-1}$ of data, and was used in the $0.9 \mathrm{fb}^{-1}$ ICHEP $2006 \mathrm{hb}$ analysis (more detail on the performance of this trigger in the analysis can be found in Section 5.4.1). The 'Muon' trigger was split into two branches in v14, one branch had the additional requirement of a L3 IP $b$-tag $<0.05$ added and the other had tighter muon cuts imposed to allow the triggers to run without pre-scales at the highest luminosities. It is planned to add these triggers into the next version of the $h b$ analysis.

\subsubsection{Conclusion}

New triggers were designed for the multi-jet Higgs analysis. The new triggers obtained a factor of four extra rejection and improved the overall efficiency by $\sim 40 \%$. The L3 IP b-tagging tool, demonstrated and tested in a trigger for the first time, allowed large gains in rejection to be achieved for very little loss in signal efficiency. The L3 IP $b$-tagging tool was measured to have an efficiency of $\sim 90 \%$ on a $3 b$-jet sample whilst reducing the background to a tenth of the level.

\subsection{Secondary Vertex Tagger}

The L3 secondary vertex tag (SVT) b-tagging tool uses tracks, jets and the PV to reconstruct secondary vertices $(\mathrm{SVs})$ in the $\mathrm{x}-\mathrm{y}$ plane in jets. The vertexing 
algorithm is described in Section 3.5.1, the optimisation and performance of the algorithm in Section 3.5.2 and the timing studies in Section 3.5.3.

\subsubsection{Vertexing Algorithm}

Due to time constraints at L3 a fast algorithm has been implemented to search for SVs. SVs are calculated using a fast impact parameter (IP) minimisation technique developed for use in the L3 vertexing tool [58].

SV 'evaluation points' (EP) are selected along the jet axis at intervals of $30 \mu \mathrm{m}$ up to a distance of $0.9 \mathrm{~cm}$ from the PV. At each SV EP the tracks not used in the PV reconstruction and which pass a track $p_{T}$ and track $\chi_{\text {dof }}^{2}$ cut $\left(\chi_{\text {Track }}^{2}\right)$ are approximated as straight lines at their distance of closest approach (DCA) to the EP. Tracks are then used if they have an IP significance with respect to the PV of greater than a threshold, $S_{\text {Track }}^{P V}$, and an IP significance with respect to the EP of less than a threshold, $S_{\text {Track }}^{S V}$.

If there is more than one track left, the $\chi^{2}$ of the vertex is calculated by minimising the $\chi^{2}$ function (the origin is centred at the EP):

$$
\chi^{2}=\sum_{a}^{N \text { tracks }} \frac{\left(d_{0}^{a}\right)^{2}}{\left(\sigma_{d_{0}}^{a}\right)^{2}}+\sum_{i=x, y} \frac{\left(j_{i}-V_{i}\right)^{2}}{\left(\sigma_{i}^{j}\right)^{2}}
$$

where $N_{\text {Tracks }}$ is the number of tracks, $\mathrm{V}$ is the vertex position, $\mathrm{j}$ is the EP position, $d_{0}$ the distance of closest approach from the track to the vertex, $\sigma_{j}$ is a jet constraint term (explained below), $a$ refers to the individual tracks and $i$ to the spatial dimensions. Figure 3.23 illustrates the process of linearising the tracks at each EP, and the quantities used in the $\chi^{2}$ minimisation. The first term is the contribution from the tracks and the second term is a jet constraint (when this method is used to calculate the PV this term corresponds to the beam spot constraint), which constrains the vertex position within the jet (see Section 3.5.1.1 for more details).

Vertices are calculated using an iterative method. The $\chi_{\text {dof }}^{2}$ of a vertex is firstly calculated using the following equation:

$$
\chi_{\text {dof }}^{2}=\frac{\chi^{2}}{2 \times N_{\text {Tracks }}-2} .
$$

Each track is removed in turn and the $\chi_{d o f}^{2}$ of the vertex recalculated. The track which causes the largest difference $\left(\delta \chi_{M a x}^{2}\right)$ in $\chi_{d o f}^{2}$ with respect to the original vertex is removed if $\delta \chi_{\text {Max }}^{2}>\chi_{C u t}^{2}$. This is repeated until either there are two tracks left or a vertex has been found which is stable, i.e. $\delta \chi_{M a x}^{2}<\chi_{C u t}^{2}$ for all tracks used to reconstruct the vertex. The $\chi_{\text {dof }}^{2}$ of the vertex at each EP is stored, and these are used to calculate the location vertices in the jet, as outlined in Section 3.5.1.2. 


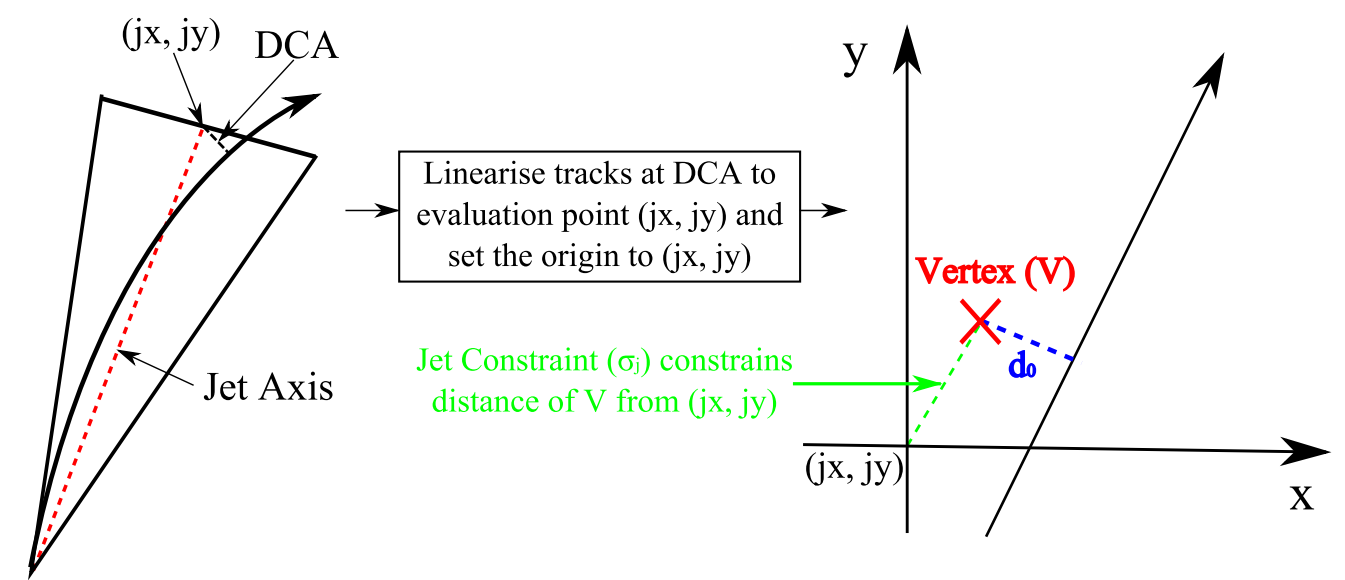

Figure 3.23: A single track in a jet which is linearised at the DCA to the EP (jx, jy). The vertex position $(\mathrm{V})$ is found by minimising the $d_{0}$ of all the tracks, with an additional constraint from $\sigma_{j}$.

\subsubsection{Jet Constraint}

Figure 3.24 (top left) shows the distance from the jet axis (dR) as a function of distance along the jet axis (dj) for offline 'Tight' SVs. Secondary vertices found within a jet are distributed around the jet axis, with an increasing spread as the distance along the jet axis increases. This relationship is used as a constraint on the vertex position in the IP minimisation. A straight line fit (Fig. 3.24 (bottom right)) to the spread of the SVs around the jet axis as a function of dj is used to parameterise the jet constraint.

\subsubsection{Vertex Identification}

The $\chi_{d o f}^{2}$ as a function of distance along the jet axis $(\mathrm{dj})$ is shown in Fig. 3.25 for a data event. There are two distinct local minima which represent two potential vertices. Local minima are defined by two low $\chi_{\text {dof }}^{2}$ values $\left(\chi_{\text {Low }}^{2}\right)$, separated by a higher $\chi_{\text {dof }}^{2}\left(\chi_{\text {High }}^{2}\right)$ value. If the two low points both have $\chi_{\text {High }}^{2}-\chi_{\text {Low }}^{2}>\chi_{\text {Split }}^{2}$, then there are two local minima and therefore two vertices.

\subsubsection{Tool Output}

The output of the tool is a list of all the secondary vertices found in the event. The vertices contain information on their decay length, decay length significance (DLS), number of tracks $\left(N_{\text {Tracks }}\right)$ and $\chi_{\text {dof }}^{2}$. The SV 'filter' (see Section 2.2.5.3) can use any of these variables to make a trigger decision. In the remainder of this chapter the DLS is used as the discriminating variable. 

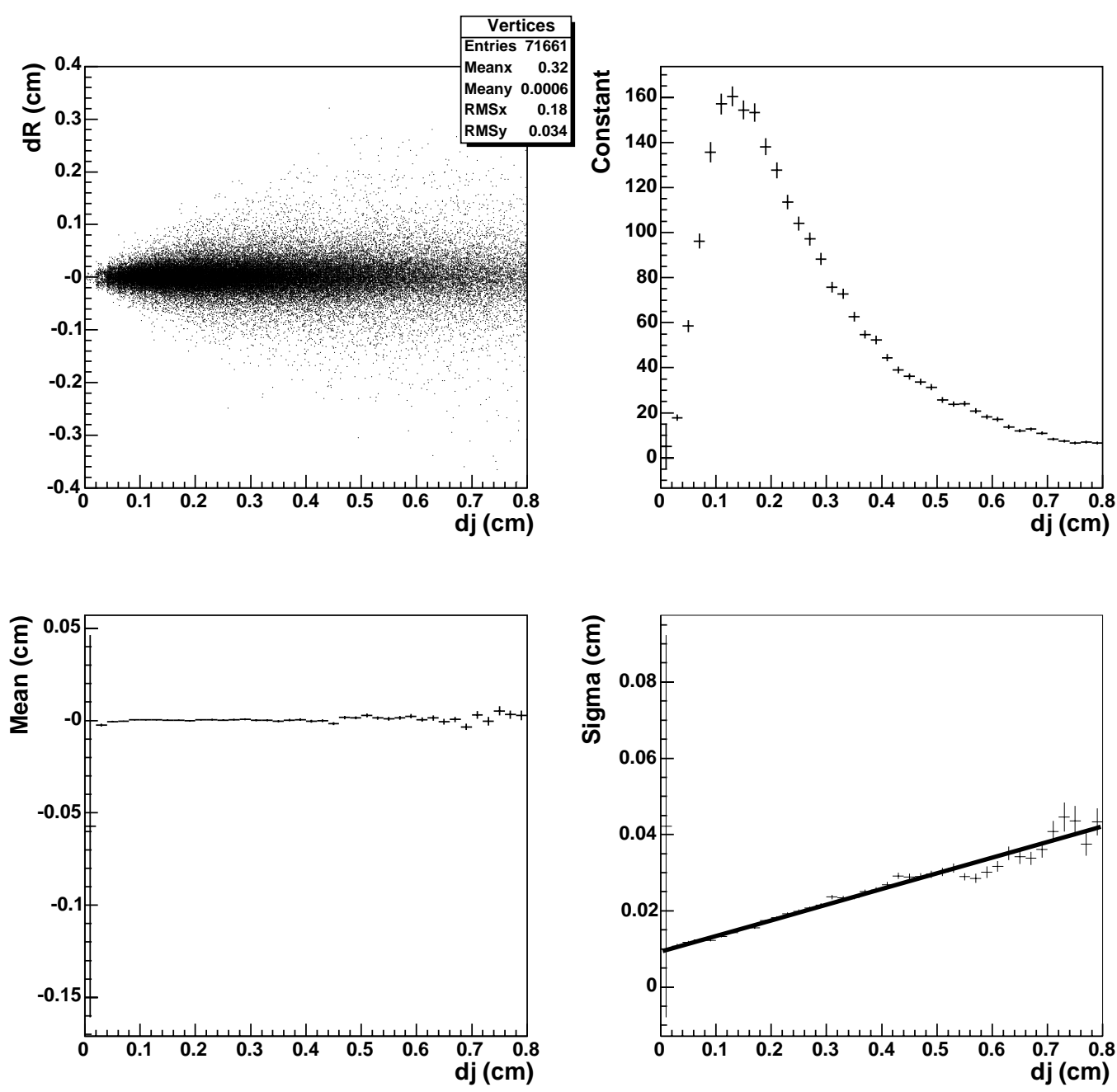

Figure 3.24: The distance from the jet axis $(\mathrm{dR})$ as a function of distance along the jet axis (dj) for offline 'Tight' SVs (top left). The distribution of SVs around the jet axis is characterised by fitting gaussian functions to slices along the jet axis. The three gaussian fit parameters, the constant (top right), mean (bottom left) and sigma (bottom right), are shown as a function of the distance along the jet axis. 


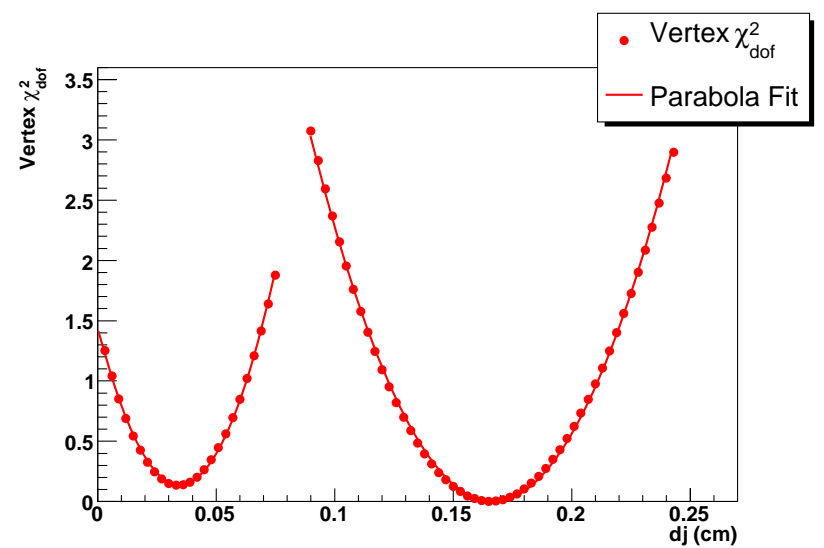

Figure 3.25: The $\chi_{d o f}^{2}$ as a function of distance along the jet axis (dj) for a 'typical' data event. Values of dj with no $\chi_{d o f}^{2}$ value occur due to there being no SVs reconstructed due to a lack of tracks.

\subsubsection{Data Optimisation}

The L3 SVT algorithm has six thresholds which need to be optimised. These are: the iterative $\chi^{2}$ threshold $\left(\chi_{\text {cut }}^{2}\right)$, the split vertex threshold $\left(\chi_{\text {split }}^{2}\right)$, PV significance threshold $\left(S_{\text {Track }}^{P V}\right)$, SV significance threshold $\left(S_{\text {Track }}^{S V}\right)$, the track $\chi_{\text {dof }}^{2}\left(\chi_{\text {Track }}^{2}\right)$ threshold and the track $\mathrm{p}_{T}$ threshold.

\subsubsection{Optimisation Strategy}

Each variable was optimised in turn whilst keeping all other variables constant. Each variable is optimised by considering the signal efficiency versus rate performance curves (defined below), produced by varying a cut on the DLS of the SVs found. The most powerful variable will be selected to provide several operating points with differing efficiencies and purities whilst the other variables will be fixed.

\subsubsection{Signal Efficiency}

The signal efficiency was measured on events (which span the data-taking period November 2002 - June 2004) which have two offline SVT tags. The signal efficiency is defined as:

$$
\text { Signal Efficiency }=\frac{\text { Number of L3 SVT Tagged Events }}{\text { Number of Signal Events }} .
$$

Signal events are defined as events with two L3 jets $E_{T}>12 \mathrm{GeV}$, a L3 PV with $|z|<35 \mathrm{~cm}$ and 2 offline SVT tags. Events are tagged by the L3 SVT tool if at least one SV, which satisfies the DLS cut, is found in the event. 


\subsubsection{Rate}

The 'rate' ${ }^{2}$ was measured on data run 188676 which was taken at an instantaneous luminosity of $\sim 15 \times 10^{30} \mathrm{~cm}^{-2} \mathrm{~s}^{-1}$. The rate is defined as:

$$
\text { Rate }=\frac{\text { Number of L3 SVT Tagged Events }}{\text { Number of Background Events }} .
$$

Background events are defined as events with two L3 jets $E_{T}>12 \mathrm{GeV}$ and a L3 PV with $|z|<35 \mathrm{~cm}$. Events are tagged by the L3 SVT tool if at least one SV is found in the event which satisfies the DLS cut. The data run, and therefore the rate, will include some events which contain real heavy flavour.

\subsubsection{Iterative Track Selection}

The iterative $\chi^{2}$ vertexing threshold removes tracks from a vertex if they contribute a $\delta \chi_{d o f}^{2}$ larger than $\chi_{C u t}^{2}$. If the threshold is set correctly it will remove tracks wrongly assigned to the vertex whilst not removing those correctly assigned.

The performance of the SVT for four representative $\chi_{C u t}^{2}$ thresholds is shown in Fig. 3.26. There is not a significant difference between the thresholds of $0.1,0.5$ and 1.0. However, a threshold of 0.5 has consistently slightly better performance than the other three operating points and so was chosen.

\subsubsection{Split Vertex}

The split vertex threshold defines the depth that a local minimum in $\chi^{2}$ along the jet axis has to be to be considered as a separate vertex. A low threshold will result in more vertices being found in a jet and a larger value will result in fewer vertices being found.

The performance of the SVT tagger for four representative split vertex thresholds is shown in Fig. 3.27. The lower the splitting threshold the higher the maximum efficiency. The optimal threshold is 0.0 .

\subsubsection{Track Primary Vertex Significance}

Tracks which originate from a SV are likely to have a large IP significance with respect to the PV. The larger the threshold on the IP significance of a track with respect to the PV, $S_{\text {Track }}^{P V}$, the purer the selection of tracks, however this will come at the expense of the efficiency. The significance of the track with respect to the

\footnotetext{
${ }^{2}$ Rate is used here in relative terms, and is therefore measured as a percentage. The term rate is used instead of background rate or background efficiency as the 'background' sample actually contains signal ( $\sim 50 \%$ of the tagged sample will be $b$-jets for a $1 \%$ fake rate operating point).
} 


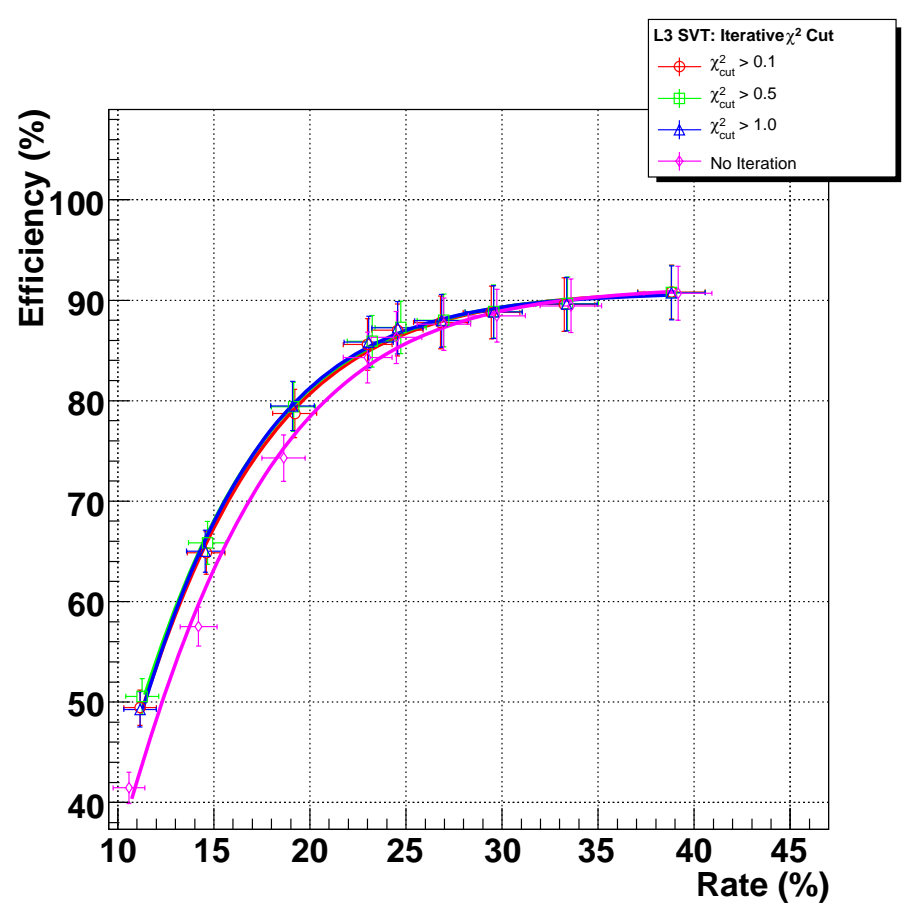

Figure 3.26: The performance of the L3 SVT $b$-tagging tool as a function of the iterative $\chi^{2}$ threshold. A representative sample of thresholds of 0.1 (red circles), 0.5 (green squares), 1.0 (blue triangles) and no iteration (purple diamonds) are shown. Errors shown are statistical.

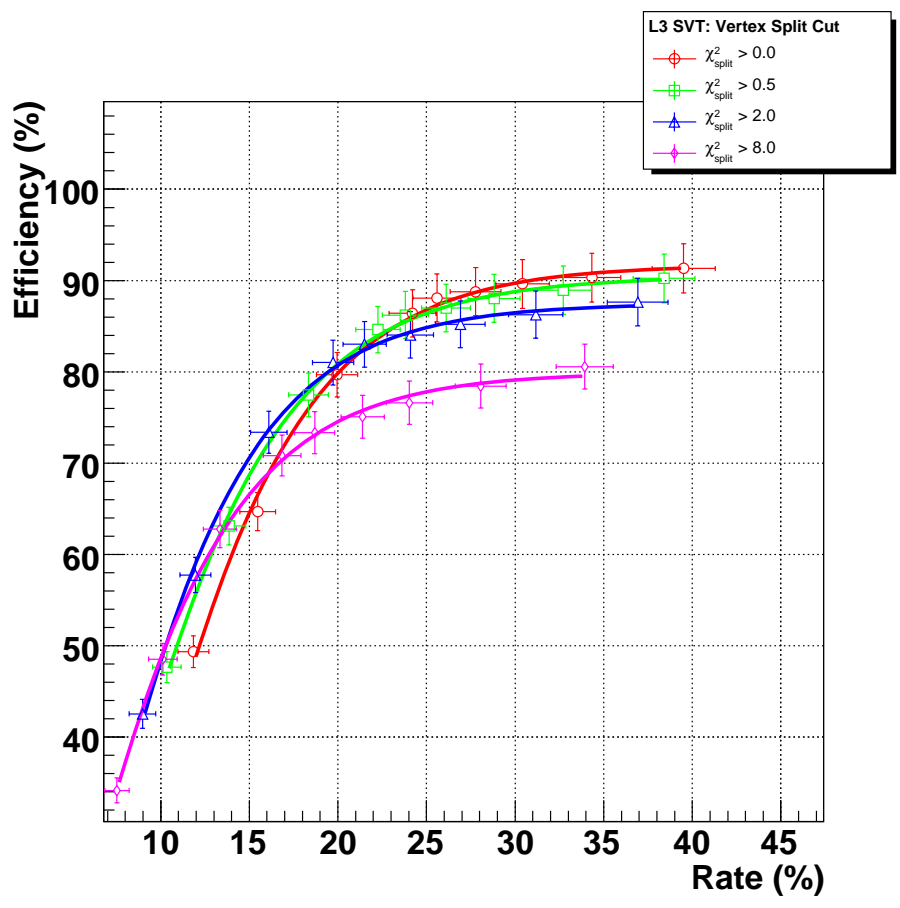

Figure 3.27: The performance of the L3 SVT $b$-tagging tool as a function of the split vertex threshold, $\chi_{\text {split }}^{2}$. A representative sample of thresholds of 0.0 (red circles), 0.5 (green squares), 2.0 (blue triangles) and 8.0 (purple diamonds) are shown. Errors shown are statistical. 
PV is calculated taking into account the primary vertex errors as well as the track errors.

The performance of the SVT tagger as a function of $S_{\text {Track }}^{P V}$ is shown in Fig. 3.28. This threshold provides a very powerful handle on the purity and efficiency of the L3 SVT tagger and allows a range of operating points to be defined with a wide performance range. For the tighter operating points a large fraction of the rate will consist of events with real heavy flavour jets.

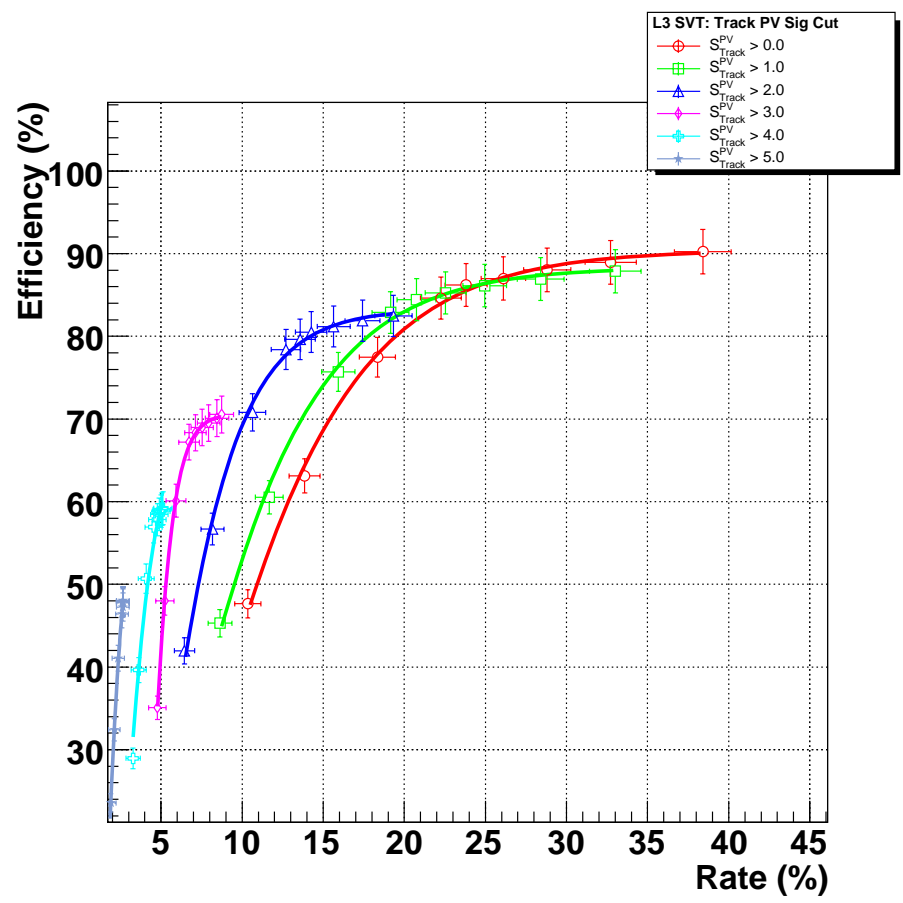

Figure 3.28: The performance of the L3 SVT $b$-tagging tool for various values of the threshold $S_{\text {Track. }}^{\text {PV }}$ The thresholds are 0 (red circles), 1 (green squares), 2 (blue triangle), 3 (purple diamond), 4 (cyan cross) and 5 (grey star). Errors shown are statistical.

\subsubsection{Track Secondary Vertex Significance}

Tracks which have a large IP significance with respect to the SV EP can be excluded from the SV reconstruction using the threshold $S_{\text {Track }}^{S V}$.

The performance of the L3 SVT tagger as a function of $S_{\text {Track }}^{S V}$ is shown in Fig. 3.29. Varying this threshold does not have a large effect on the performance. Tightening the threshold shifts the performance curve to a position with a lower efficiency and rate. 


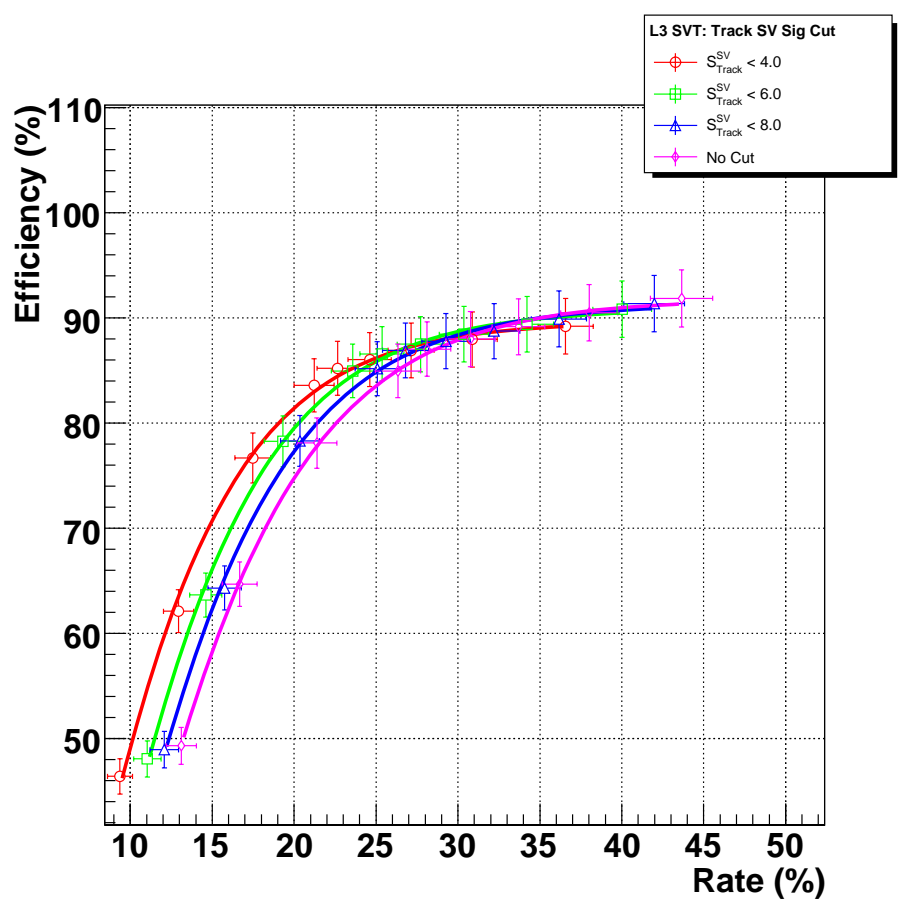

Figure 3.29: The performance of the L3 SVT $b$-tagging tool for various values of the threshold $S_{\text {Track. }}^{S V}$. The thresholds are 4 (red circles), 6 (green squares), 8 (blue triangles) and no threshold (purple diamonds). Errors shown are statistical.

\subsubsection{Track $\chi_{\text {dof }}^{2}$}

The track $\chi_{d o f}^{2}$ is an important indicator of the quality of the track. A tight threshold on the $\chi_{d o f}^{2}$ of the tracks will reduce the contribution from fake and badly reconstructed tracks; however this comes at the expense of the tracking efficiency.

The performance of the SVT tagger for track $\chi_{d o f}^{2}$ thresholds from 1.0 to 5.0 is shown in Fig. 3.30. A sufficiently tight value for this threshold shifts the performance of the SVT tagger to a less efficient but purer operating point.

\subsubsection{Track $\mathrm{p}_{T}$}

Varying the $\mathrm{p}_{T}$ of tracks used in the SV reconstruction provides a handle on the quality of the tracks. The performance of the SVT tagger for track $\mathrm{p}_{T}$ thresholds from 0.5 to $1.5 \mathrm{GeV}$ is shown in Fig. 3.31. Varying this threshold alters the efficiency and purity of the tagger. A high $\mathrm{p}_{T}$ threshold results in a less efficient but purer tagger.

\subsubsection{Optimised Variable Selection}

The final optimised variable selection is shown in Table 3.6. The PV significance threshold is the most powerful handle on the performance of the SVT tagger and will 


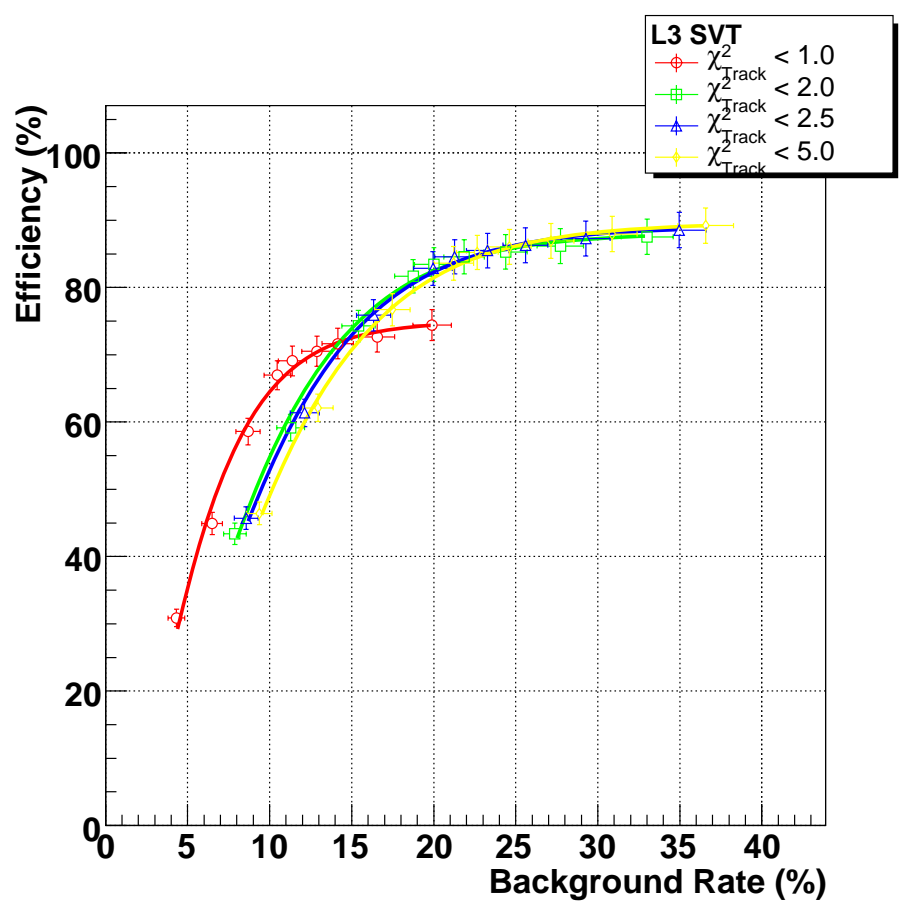

Figure 3.30: The performance of the L3 SVT $b$-tagging tool for various track $\chi_{d o f}^{2}$ thresholds. The lines represent the thresholds 1.0 (red circles), 2.0 (green squares), 2.5 (blue triangles) and 5.0 (purple diamonds). Errors shown are statistical.

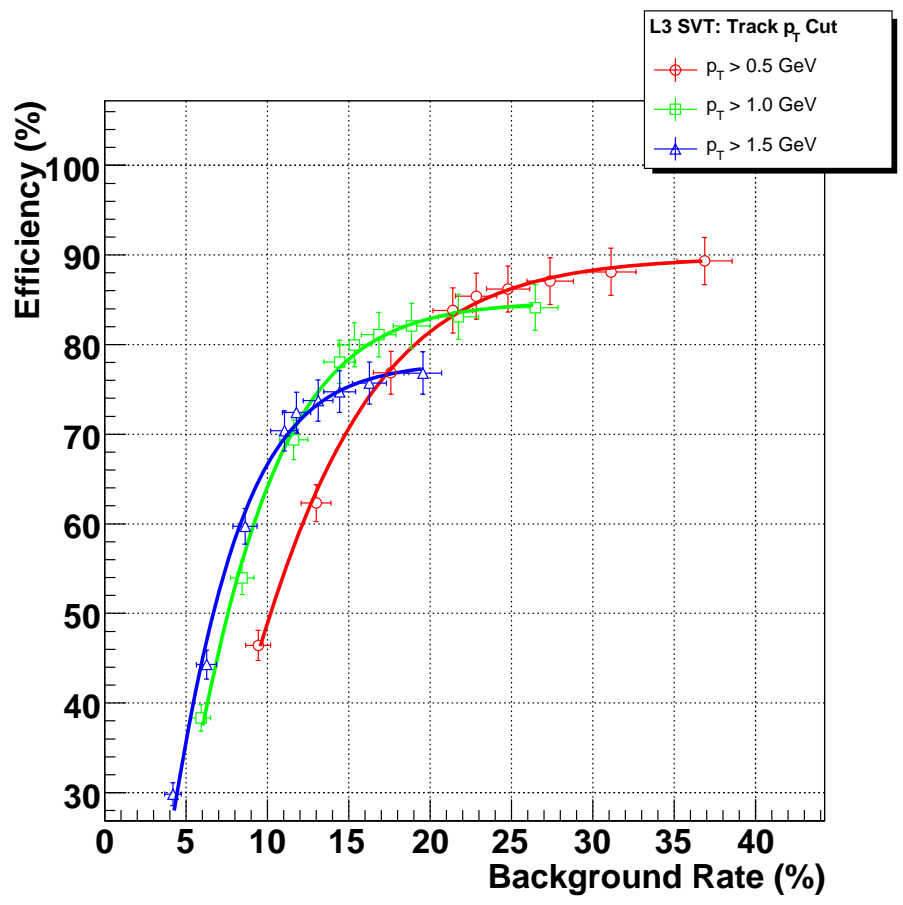

Figure 3.31: The performance of the L3 SVT $b$-tagging tool for various track $\mathrm{p}_{T}$ thresholds. The lines represent the thresholds 0.5 (red circles), 1.0 (green squares) and $1.5 \mathrm{GeV}$ (blue triangles). Errors shown are statistical. 
therefore be used to define several operating points. The remaining thresholds are selected to produce the highest efficiency. If two values produced a similar efficiency then the one with the lowest rate was chosen as the optimal value.

\begin{tabular}{|c|c|}
\hline Variable & Optimised Value \\
\hline Iterative $\chi_{d o f}^{2}$ & 0.5 \\
Split $\chi_{d o f}^{2}$ & 0.0 \\
Track $\chi_{d o f}^{2}$ & 5.0 \\
Track $\mathrm{p}_{T}$ & $0.5 \mathrm{GeV}$ \\
Track SV Significance & 8.0 \\
Track PV Significance & Varied \\
\hline
\end{tabular}

Table 3.6: The final optimised thresholds used in the L3 SVT $b$-tagging tool

The performance for the optimised SVT b-tagging tool is shown in Fig. 3.32. Representative signal efficiencies and rates for each of the operating points are shown in Table 3.7.

\begin{tabular}{|c|c|c|c|}
\hline Name & $S_{\text {Track }}^{\text {PV }}$ & Efficiency (\%) & Rate (\%) \\
\hline PV0 & 0 & 93 & 43 \\
PV1 & 1 & 89 & 25 \\
PV2 & 2 & 82 & 15 \\
PV3 & 3 & 75 & 10 \\
PV4 & 4 & 63 & 6 \\
PV5 & 5 & 51 & 4 \\
\hline
\end{tabular}

Table 3.7: Representative signal efficiencies and rates for the six operating points defined for the L3 SVT $b$-tagging tool.

\subsubsection{Vertex Parameter}

A comparison of the SV variables, namely: DLS, $\chi_{\text {dof }}^{2}$, decay length, $N_{\text {Tracks }}$ and the number of vertices for the signal and background samples is shown in Fig. 3.33. The SVs in the signal and background samples demonstrate the characteristics of $b$ and light-jets respectively. The separation between the variables in the signal and background samples would be suitable for use in a neural network, which could potentially further improve the $b$-tagging performance. 


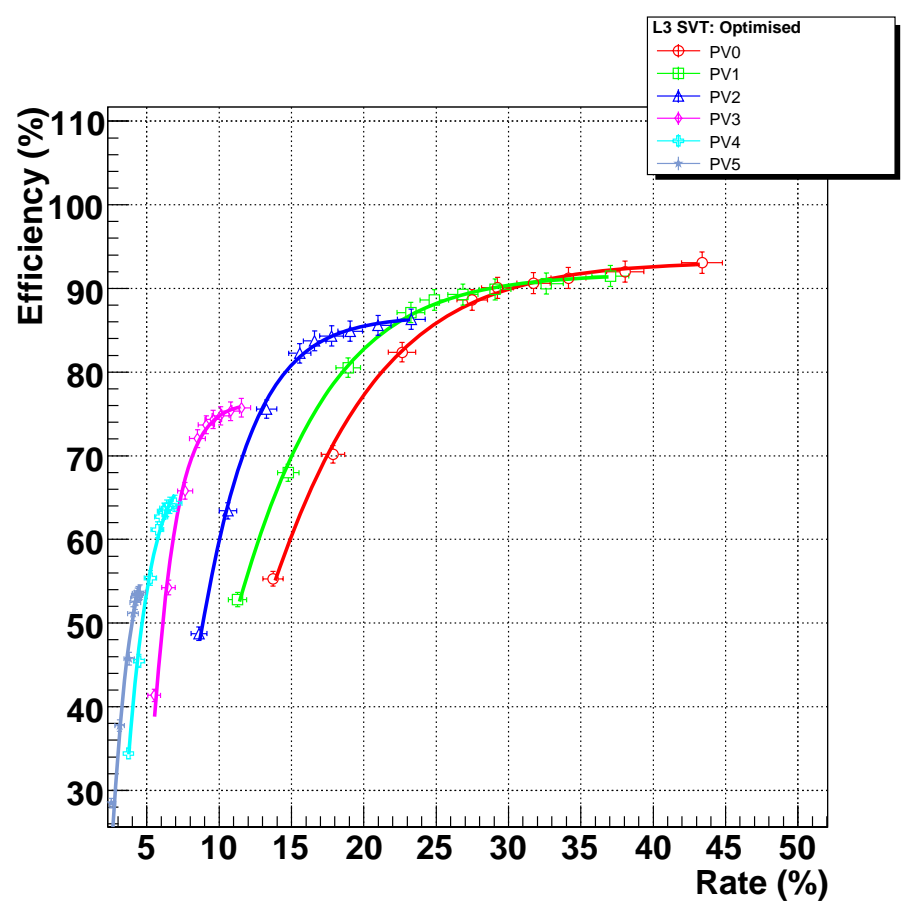

Figure 3.32: The performance of the optimised L3 SVT $b$-tagging tool for the six operating points: PV0 (red circles), PV1 (green squares), PV2 (blue triangles), PV3 (purple diamonds), PV4 (cyan crosses) and PV5 (grey stars). Errors shown are statistical. 

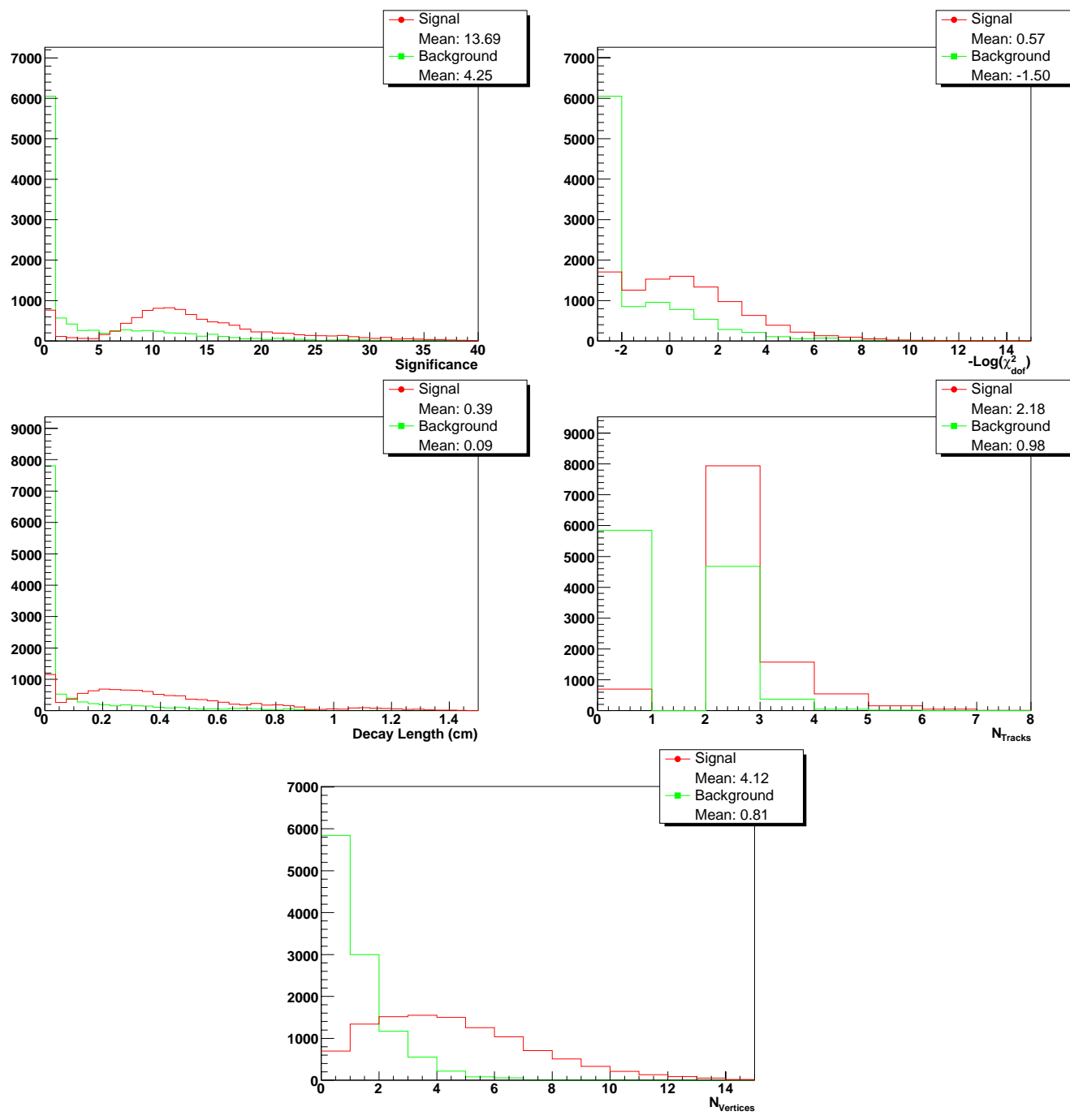

Figure 3.33: The DLS (top left), - $\log \left(\chi_{\text {dof }}^{2}\right)$ (top right), decay length (middle left), $N_{\text {Tracks }}$ (middle right) and the number of vertices (bottom) for the signal sample (red line) and background sample (green line) for the operating point PV0. 


\subsubsection{Timing Studies}

Due to the limited time available at L3 the time taken by each tool needs to be carefully studied. Timing tests are carefully conducted on the same CPU and under the same conditions to ensure consistency between different timing measurements - in this case a $2.4 \mathrm{GHz}$ Linux machine with no other jobs running. The timing results are shown in Table 3.8 and in Fig. 3.34.

The time taken as a function of the number of tracks in the event is shown in Fig. 3.35. There is an approximately linear dependency between the time taken and the number of tracks.

\begin{tabular}{|c|c|c|}
\hline \multirow{2}{*}{ Tool } & \multicolumn{2}{|c|}{ Timing $(\mathrm{ms})$} \\
\cline { 2 - 3 } & Signal & Background \\
\hline SMT Unpacker & 5.2 & 5.2 \\
CFT Unpacker & 2.7 & 2.3 \\
Tracker & 203.2 & 93.2 \\
Primary Vertexing & 0.6 & 0.3 \\
Secondary Vertexing & 3.4 & 1.4 \\
\hline
\end{tabular}

Table 3.8: The time taken by the L3 SVT $b$-tagging tool, and the tracking and vertexing tools for comparison, in the signal and background samples. Errors on the times were estimated to be $\sim 10 \%$ from repeated measurements.

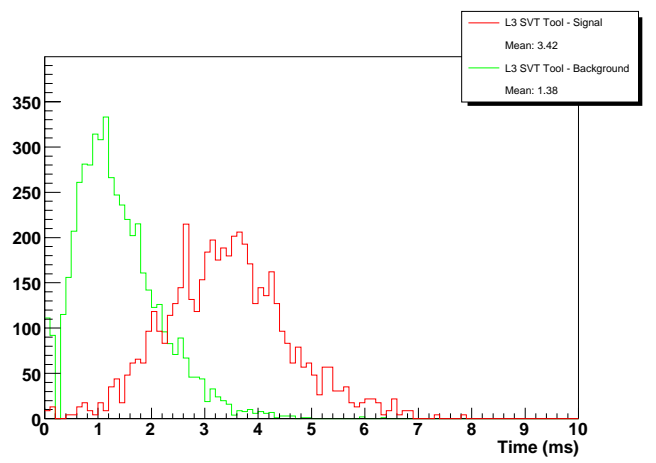

Figure 3.34: The timing distributions for the L3 SVT $b$-tagging tool operating point PV0 on the signal events (red line) and background events (green line).

\subsubsection{Conclusion}

A new $b$-tagging tool which locates displaced secondary vertices in jets has been developed and optimised for use in the L3 trigger. The new tool has a large potential for reducing background rates at little expense to the trigger efficiency. Six potential 


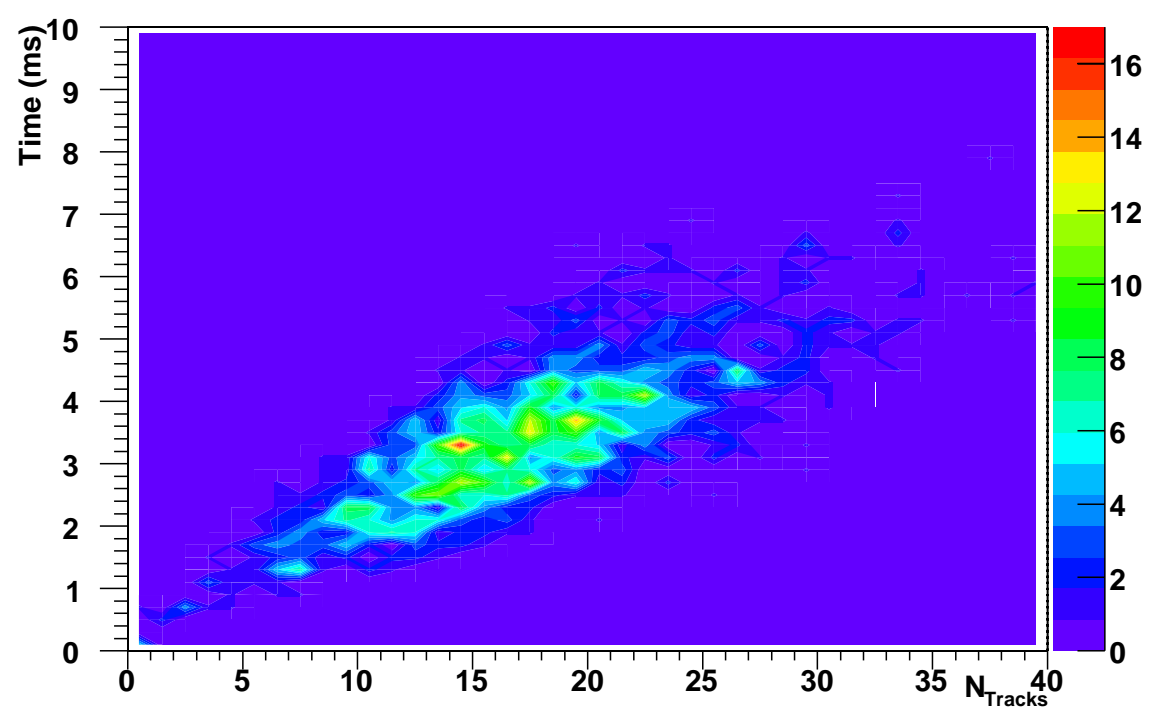

Figure 3.35: The time taken as a function of the number of tracks in the signal events for the operating point PV0.

operating points have been identified for use; the points have signal efficiencies varying from $93 \%$ to $54 \%$ for rates ranging from $43 \%$ to $4 \%$. The tool's time consumption was measured as $1.4 \mathrm{~ms}$ at an instantaneous luminosity of $\sim 15 \times 10^{30} \mathrm{~cm}^{-2} \mathrm{~s}^{-1}$ for the PV0 operating point (which has the highest time consumption). The SVT tool will now be combined with the IP $b$-tagging tool and the individual and joint results will be commissioned for use in the next version of the trigger list. 


\section{Chapter 4}

\section{The Neural Network $b$-Tagger}

\subsection{Introduction}

$b$-jets are a key signature of a wide range of physical processes of interest, including many Higgs search channels. The ability to accurately identify $b$-jets is vital in reducing the otherwise overwhelming background to these channels from processes involving light-jets.

This chapter covers the development and testing of the first (and currently only) Neural Network (NN) $b$-tagging tool at DØ. The new $b$-tagging tool has significantly enhanced performance compared to the other $b$-tagging tools available, and consequently has become the default $b$-tagging tool at D $\varnothing$. The improved tagging has significantly increased the sensitivity of the Higgs search at DØ. The benefit from the new tagger is equivalent to a doubling of the luminosity [72].

Two main components of the study of the NN tagger are covered in this chapter. Firstly, the development of the NN tagger, which includes the selection of input variables, optimisation of the NN and the performance on Monte Carlo (MC). Secondly, the measurement and testing of the performance on data, referred to as the 'certification' of the tagger. The certification procedure is necessary due to the different tagging performance in $\mathrm{MC}$ and data, and all taggers must be 'certified' before they can be used in any analyses shown external to DØ.

An introduction to the certification procedure, along with other important concepts, is outlined in the remainder of this introduction. Full details of the development and certification of the NN tagger can be found in [73] and [74] respectively. The input variables tested are outlined in Section 4.2, the optimisation of the NN on $\mathrm{MC}$ in Section 4.3, the MC performance in Section 4.4, a comparison of the MC and data inputs in Section 4.5, the b-efficiency measurement in Section 4.6, the fake rate 
measurement in Section 4.7 and the overall performance on data in Section 4.8. The development of the NN tagger was all the author's own work, and the 'certification' of the tagger was carried out with the help of another $\mathrm{PhD}$ student.

\subsubsection{Procedure for Data Certification}

The tracking in the p14 MC simulation of the $\mathrm{D} \varnothing$ detector is not an entirely realistic simulation of data. The tracking is over optimistic in both the quality and number of tracks found. This results in the $b$-tagging efficiency in MC being over estimated by $10-20 \%$ when compared to data [74].

b-tagging is applied to MC and data differently, as is outlined in Fig. 4.1, due to the differences in the $b$-tagging. To accurately $b$-tag jets in MC tag rate functions (TRFs) for $b, c$ and fake-jets ${ }^{1}$ need to be measured for data at various 'operating points'. An operating point (OP) is a version (or cut on the output) of a tagging tool which has a particular fake rate. b-tagging in data and MC can only therefore be carried out at particular OPs which have been measured on data.

The $b$-tagging is run directly on the data, and the jets either pass or fail at a particular operating point. For MC, the TRFs measured for the operating point are used to predict the probability that the $b, c$ and fake-jets in the events are tagged. The probabilities for each of the jets are combined into an event weight which represents the probability that the event passed the tagging criteria. The weighted MC sample is equivalent to the b-tagged data sample. Four functions are used in the $b$-tagging correction:

Scale Factor (SF) - The factor by which the $b$ and $c$ MC tagging efficiencies have to be multiplied by to obtain the data tagging efficiencies.

$\mathbf{T R F}_{b}$ - The efficiency to tag a $b$-jet in data.

$\mathbf{T R F}_{c}-$ The efficiency to tag a $c$-jet in data.

Fake-Tag Rate (FTR) ${ }^{2}$ - The efficiency to tag a fake-jet in data.

These functions are parameterised in terms of the $\mathrm{p}_{T}$ and $\eta$ of the jets. The NN tagger's performance on data was measured following the $b$-ID group's standard certification procedure [75]. Techniques developed and employed in the Jet Lifetime Impact Parameter (JLIP) [76] and the Secondary Vertex Tag (SVT) [77] Pass2 certifications were used in this certification and developed further. The concepts outlined in the following sections are integral to the certification of the NN tagger.

\footnotetext{
${ }^{1}$ The term fake-jets refers to $u d s$ and gluon-jets.

${ }^{2}$ This could also be referred to as the light-jet TRF.
} 


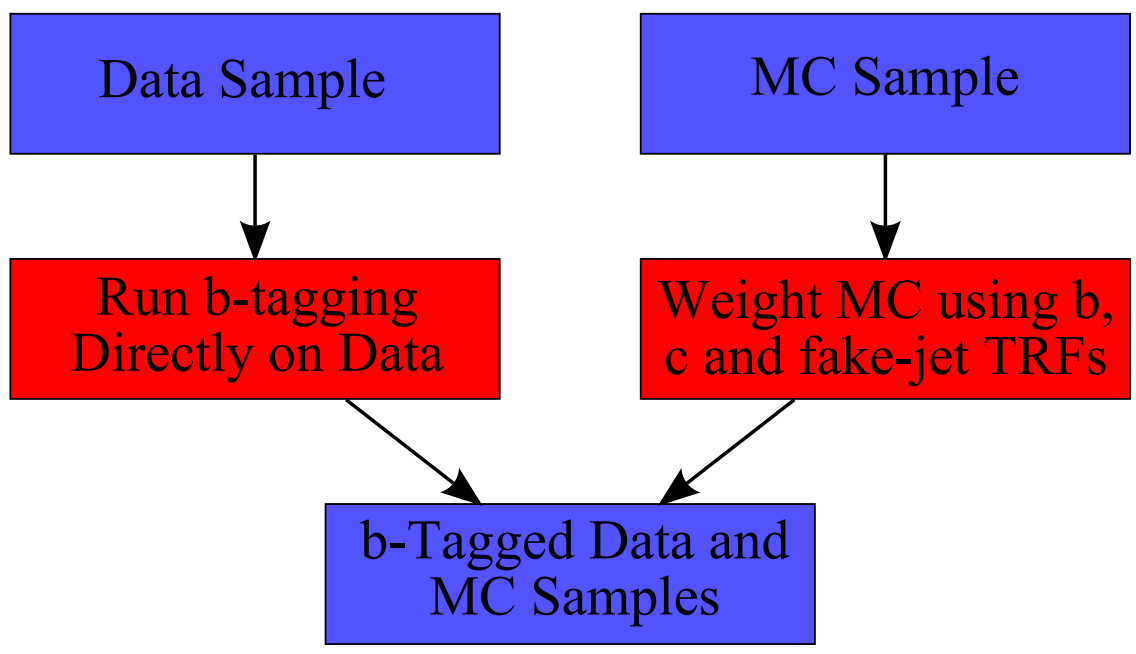

Figure 4.1: The two methods used to apply $b$-tagging to MC and data.

\subsubsection{Operating Points}

In the certification, the NN tagger has its performance evaluated at various OPs. Six OPs were chosen, to give fake rates on data close to those outlined in Table 4.1.

\begin{tabular}{|c|c|c|c|c|c|c|}
\hline Name & Tight & Medium & Loose & L2 & L3 & L4 \\
\hline NN Cut & $>0.775$ & $>0.65$ & $>0.5$ & $>0.325$ & $>0.25$ & $>0.2$ \\
\hline Fake Rate (\%) & 0.3 & 0.5 & 1.0 & 2.0 & 3.0 & 4.0 \\
\hline
\end{tabular}

Table 4.1: The NN tagger's OPs with their corresponding design fake rates.

\subsubsection{Negative and Positive Tags}

The majority of fake-tags are due to resolution effects affecting the reconstruction of the PV and tracks. The impact parameters (IP) of such tracks should be symmetrically distributed around zero (there will actually be slightly more with positive IP due to some long lived 'light' particles), whereas tracks from $b$ and $c$ particles will predominantly have positive IPs due to their long lifetimes.

Taggers use the symmetrical distribution of the track IPs from fake-jets and the asymmetrical distribution from heavy-flavour jets to construct two types of tag. Tags constructed from tracks with negative IPs have very little contribution from heavy flavour and therefore approximately describe the fake rate. Tags constructed from tracks with positive IPs will contain the vast majority of the heavy flavour signal, with only approximately half the component of fake-tags. 
Each tagger provides negative tag (NT) and positive tag (PT) results for each jet. The definition of what constitutes a NT result varies for each tagger and is detailed below:

CSIP NT - Calculated from tracks which have a negative IP significance with respect to the primary vertex.

JLIP NT - Calculated from tracks which have a negative IP significance with respect to the primary vertex.

SVT NT - Secondary vertices (SV) which have a negative decay length and a $d R<0.5$ with respect to the jet.

For the NN tagger, a NT result is defined as the output from the NN when all of the NN inputs come from the NT results for the individual taggers.

\subsubsection{Away Tag}

An 'away tag' is an indirect method of tagging a jet without actually performing any cuts on the jet and thereby biasing the jet sample. A jet is defined as having an away tag if it is in an event with exactly two jets, and the other jet has passed some $b$-tagging criteria. An away-tagged jet has a considerably enhanced probability of being heavy flavour.

\subsubsection{Calorimeter Regions}

It is sometimes useful when undertaking performance studies to consider the $\mathrm{D} \emptyset$ detector in three distinct $\eta$ regions which have different detector responses and tracking capabilities: the Central Calorimeter (CC), Inter-Cryostat Region (ICR) and the End Cap (EC) as outlined in Table 4.2.

\begin{tabular}{|c|c|}
\hline Calorimeter Region & Physical $\eta$ \\
\hline Central Calorimeter (CC) & $0<|\eta|<1.2$ \\
Inter-Cryostat Region (ICR) & $1.2<|\eta|<1.8$ \\
End Cap (EC) & $1.8<|\eta|<2.4$ \\
\hline
\end{tabular}

Table 4.2: Calorimeter regions (which have different detector responses) in the $\mathrm{D} \emptyset$ detector. 


\subsubsection{Statistical Errors}

Statistical errors are accounted for in the certification by calculating \pm 1 sigma curves which represent the \pm 1 sigma deviation from the central fit value. The deviation of the fit from the central value is calculated at each point of the fit function using:

$$
\Delta f=\sum_{i}^{n} \sum_{j}^{n} E_{i j} \frac{\partial f}{\partial p_{i}} \frac{\partial f}{\partial p_{j}}
$$

where $\Delta f$ is the change in the central fit value, $E_{i j}$ is the error matrix for the parameters, $i$ and $j$ relate to the fit parameters, $n$ is the number of fit parameters and the derivatives are calculated with respect to the appropriate fit parameters.

\subsubsection{Neural Networks}

A Root based Neural Network (NN) package, TMultiLayerPerceptron [78], which was inspired by the mlpfit package [79] was used to construct the NN. For the training stage the NN package takes as input a tree of the examples to be trained upon, which in this instance is jets.

A multilayer perceptron (MLP) NN is a simple feed-forward network which consists of a layer of input nodes, one or more layers of hidden nodes and one layer of output nodes. The nodes, called neurons, are connected to each of the nodes in the consecutive layers by links called synapses that have a weight $w_{j}$ and bias $w_{0}$ representing the strength of the signal between the two nodes. The neuron $j$ of the hidden or output layer computes a linear combination $x_{j}$ of the neurons in the previous layer $y_{i}$ with a bias:

$$
x_{j}=w_{0 j}+\sum_{i} w_{i j} y_{i} .
$$

The output $z_{j}$ of the neuron $\mathrm{j}$ is then a function of the input $x_{j}$. The function is either linear:

$$
z_{j}=x_{j}
$$

or a sigmoid function

$$
z_{j}=\frac{1}{1+e^{-x_{j}}}
$$

depending on the layer. The different layers carry out the following operations.

Input Nodes - Receive their input from the external sample which is scaled and then output to the nodes in the first hidden layer. 
Hidden Layers - Output a sigmoid function of a linear combination of the outputs from the nodes in the previous layer.

Output Layer - Creates a linear combination of the outputs from the nodes in the previous layer which is then an output of the NN.

A NN is a linear combination of sigmoid functions and constructed in such a way as to take advantage of two very important theorems involving the computation of linear combinations of sigmoid functions.

1. A linear combination of sigmoid functions can approximate any continuous function [80].

2. When trained with a desired output of 1 for signal and 0 for background, the output for a set of inputs is, approximately, the probability that the inputs are signal [81].

Initially the weights for each of the synapses are set randomly between -0.5 and 0.5. The NN output, $o_{p}$, is compared to the desired output, $t_{p}$, on a set of examples p. The training algorithms try to minimise the error on the training samples by altering the weights. The total error, $E$, on the training samples (training error) is given by

$$
E=\sum_{p} \frac{1}{2} \omega_{p}\left(o_{p}-t_{p}\right)^{2},
$$

where $\omega_{p}$ is an event weight. All the training algorithms compute the first order derivative of the error with respect to the weights

$$
\frac{d E_{p}}{d w_{i j}}=\sum_{p} \frac{d e_{p}}{d w_{i j}}
$$

where $p$ is the set of examples and $e_{p}$ is the error on each example. This is called back-propagation of the errors. A loop over all the examples is called an epoch. There are six learning methods implemented. They are:

Stochastic - Uses the Robbins-Monro stochastic approximation method to update the weights after each input example [82].

Batch - Is the same as the stochastic algorithm but the weights are only updated after considering all the input examples. 
Line Search Methods - The following algorithms all rely on the theory of unconstrained minimisation [83]. Each of these methods works in a similar manner. For a set of examples $t$ :

1. A direction $\vec{s}_{t}$ is computed from the gradient $\nabla \mathrm{E}$.

2. The $\alpha$ which minimises $E\left(\overrightarrow{w_{t}}+\alpha \overrightarrow{s_{t}}\right)$ is found (this part is called the line search).

3. The weights are updated by $\overrightarrow{w_{t+1}}=\overrightarrow{w_{t}}+\alpha \overrightarrow{s_{t}}$

4. Goto 1)

The variations of the line search method differ in the way that step 1) is carried out. The simplest is the steepest descent [83] algorithm where $\vec{s}_{t}=-$ $\nabla(E)$. There are two conjugate gradient methods using the Polak-Ribiere [83] and Fletcher-Reeves [83] updating formulas. The Broyden, Fletcher, Goldfarb, Shanno (BFGS) algorithm relies upon the computation of the second derivatives of the error with respect to the weights, and uses the first and second derivatives in a quasi-Newton fitting method [83].

\subsubsection{Data and MC Samples}

The data samples used are derived from the $b$-ID, EM1TRK (EM) and QCD 'skims'3 consisting of data taken from July 2002 to August 2004 [84]. The skimming criteria are outlined with the number of events in Table 4.3. The skims were processed excluding bad calorimeter data and runs marked as bad in the runs quality database [65]. A larger skim (COMB) combining all the selected jets in the EM and QCD skims was also created. Any overlap between the EM and QCD skims was assumed to be small, with any corresponding effect on the fake rates to be negligible.

The MC samples used are outlined in Table 4.4. All the jets from the different MC samples were 'combined' into large samples of jets, which are referred to as the $c, b$, muonic $c$ and muonic $b$-jet samples in the rest of the text. The two types of sample are typically referred to as muonic and inclusive jet samples. Muonic jets are those with a muon within the jet (semi-leptonic decays), and inclusive jets contain both the semi-leptonic and the hadronic decays (i.e. all $b$-jets). The distinction is important as the two types of jets have different $b$-tagging properties. The different $\mathrm{p}_{T}$ QCD jet samples were also merged into continuous $\mathrm{p}_{T}$ samples using the fall off

\footnotetext{
${ }^{3}$ Subsets of data, known as skims, are produced for use in similar analyses by 'skimming' the data for events which fulfill specific criteria.
} 
in jet $\mathrm{p}_{T}$, as seen in data, to appropriately weight the different samples. The number of jets in each of these samples is outlined in Table 4.5. In the remainder of this chapter the $b, c$ and fake-jet samples produced from the different MC samples are referred to using the notation $X \rightarrow j$, where $X$ is the MC sample name and $j$ is the jet flavour. For example $t \bar{t} \rightarrow b \rightarrow \mu$ is the notation for the sample of muonic $b$-jets from the $t \bar{t}$ sample.

\begin{tabular}{|c|c|c|}
\hline Skim & Skim Criteria & Events \\
\hline EM & 1 Electron with $p_{T}>4 \mathrm{GeV}$ & $64 \mathrm{M}$ \\
\hline QCD & Jet Triggers & $58 \mathrm{M}$ \\
\hline COMB & Combination of the QCD and EM skims & $122 \mathrm{M}$ \\
\hline$b$-ID & One loose $\mu$, with a $p_{T}>4 \mathrm{GeV}$ inside a 0.7 cone jet & $91 \mathrm{M}$ \\
\hline
\end{tabular}

Table 4.3: Data skims used in the certification of the NN tagger.

\begin{tabular}{|c|c|}
\hline Sample & Number of Events \\
\hline$t \bar{t}$ & 390,000 \\
$Z \rightarrow b \bar{b}$ & 200,000 \\
$Z \rightarrow b \bar{b}$ with $\mu$ & 100,000 \\
$Z \rightarrow c \bar{c}$ & 210,000 \\
$Z \rightarrow c \bar{c}$ with $\mu$ & 100,000 \\
$Z \rightarrow q \bar{q}$ & 250,000 \\
QCD $p_{T}=20-40,40-80,80-160,160-320$ & $1,160,000$ \\
QCD $b \bar{b} p_{T}=20-40,40-80,80-160,160-320$ & 570,000 \\
QCD $c \bar{c} p_{T}=20-40,40-80,80-160,160-320$ & 620,000 \\
\hline
\end{tabular}

Table 4.4: $\mathrm{MC}$ samples used in the development and certification of the NN tagger. All the samples were generated using Pythia [70], except for the $t \bar{t}$ sample which was generated using Alpgen [85] before being processed through Pythia to simulate the hadronisation and showering.

\subsection{Input Variables}

The following variables are potentially good discriminators between fake and $b$ jets. All the following plots are from the merged QCD MC samples, and the input variables are from the standard $\mathrm{D} \varnothing$ taggers. 


\begin{tabular}{|c|c|c|}
\hline Flavour & Sample & Number of Jets \\
\hline inclusive $b$ & $b$ (Combined) & $1,800,000$ \\
inclusive $b$ & QCD $b \bar{b}$ (Merged) & 270,000 \\
\hline muonic $b$ & $b \rightarrow \mu$ (Combined) & 180,000 \\
\hline inclusive $c$ & $c$ (Combined) & $1,500,000$ \\
inclusive $c$ & QCD $c \bar{c}$ (Merged) & 300,000 \\
\hline muonic $c$ & $c \rightarrow \mu$ (Combined) & 90,000 \\
\hline$u d s g$ & $Z \rightarrow q \bar{q}$ & 375,000 \\
$u d s g$ & QCD Fake (Merged) & 500,000 \\
\hline$u d s c b g$ & QCD (Combined) & $1,100,000$ \\
\hline
\end{tabular}

Table 4.5: Number of MC jets of each flavour available in each of the combined and merged samples after data processing and jet selection.

\subsubsection{JLIP Variables}

The variables of interest from the JLIP tagger [76] are listed below and shown in Fig. 4.2.

JLIP Prob - The probability that the jet originated from the PV. The closer to 0 the more likely that the jet was a $b$-quark. If there is not enough information in the event to calculate a probability this value is set to 1 .

JLIP Prob $_{\text {Red }}$ - The JLIP probability re-calculated with the most $b$-like track removed from the calculation. This identifies jets where a mismeasured or fake track causes a mistag of the jet. If there is not enough information to calculate the reduced probability the value is set to 1 .

JLIP $N_{\text {Tracks }}$ - The number of tracks used in the JLIP probability calculation. $b$ jets will typically have more tracks than fake-jets for a give $\mathrm{p}_{T}$.

\subsubsection{SVT}

The SVT [77] tagger finds secondary vertices (SV). As multiple SVs can be found in each jet, SVs are ranked in order of their most powerful discriminator, the decay length significance (DLS). The SV with the largest DLS within a jet is used to provide the SVT input variables. Potential variables from the Loose SVT $\left(\mathrm{SVT}_{L}\right)$ tagger are listed below and shown in Fig. 4.3. If no secondary vertex is found, the 

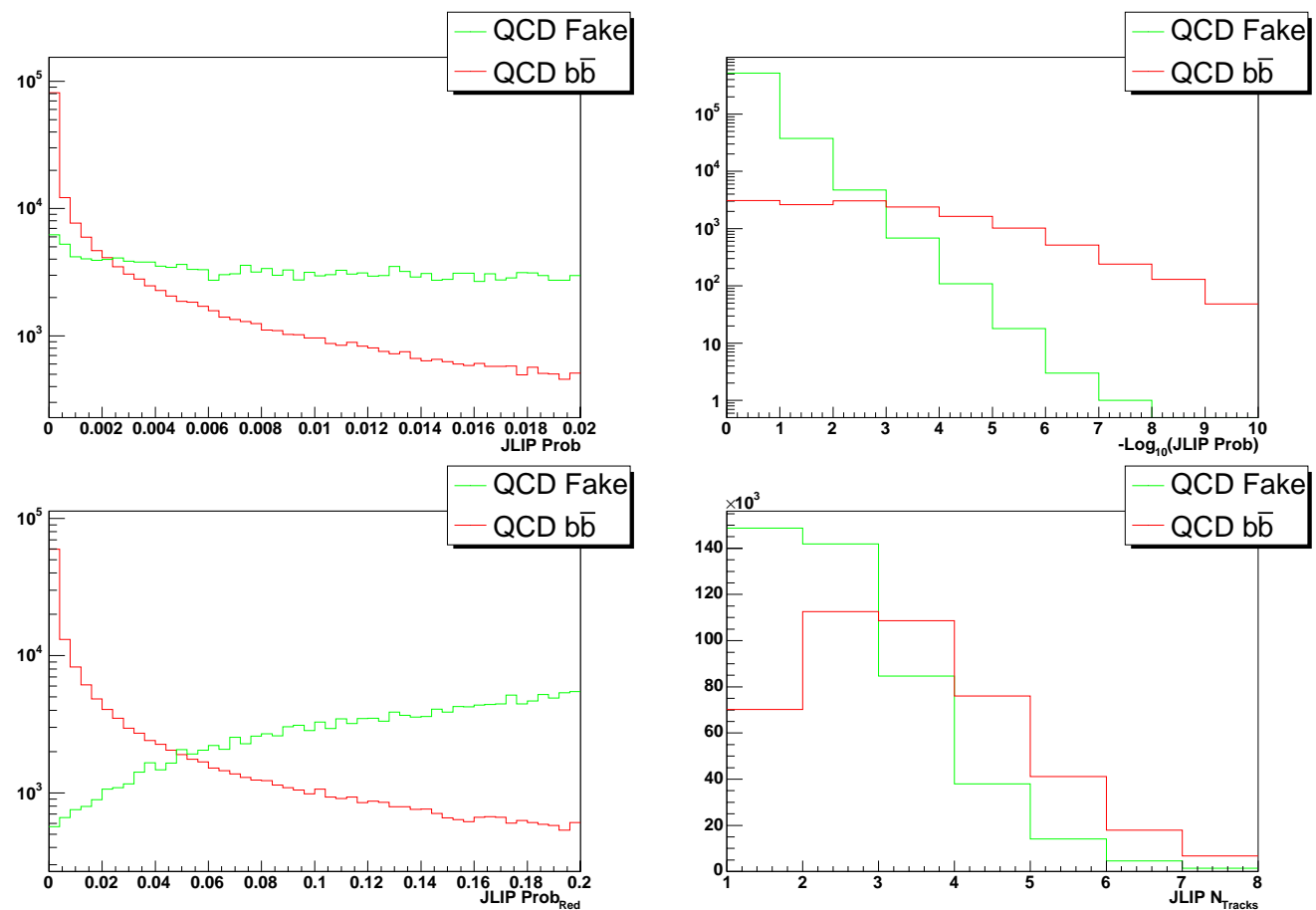

Figure 4.2: JLIP tagger variables Prob (top left), $-\log _{10}$ (Prob) (top right), Prob ${ }_{R e d}$ (bottom left) and the $N_{\text {Tracks }}$ (bottom right) for QCD $b \bar{b}$ (red) and fake (green) MC jets. See the text for the full definitions of variables.

SVT values are set to 0 , apart from the SVT $\chi_{d o f}^{2}$ which is set to 75 corresponding to the upper bound of $\chi_{d o f}^{2}$ values.

SVT $D L S$ - The decay length significance of the secondary vertex with respect to the primary vertex.

SVT $\chi_{d o f}^{2}$ - The $\chi^{2}$ per degree of freedom of the secondary vertex.

SVT $N_{\text {Tracks }}$ - The number of tracks used to reconstruct the secondary vertex.

SVT Mass - The mass of the secondary vertex. Calculated from the combined rest mass of the tracks assuming all tracks were pions.

SVT Num - The number of secondary vertices reconstructed in the jet.

SVT $d R$ - The dR between the vertex and the jet axis.

The standard SVT tagger operating points are not ideal for use in a NN. They find a very pure selection of secondary vertices, but, if SVT reconstruction in a jet fails, no information is available. This results in no information being available for a large number of the jets. A NN performs best with the maximal amount of 

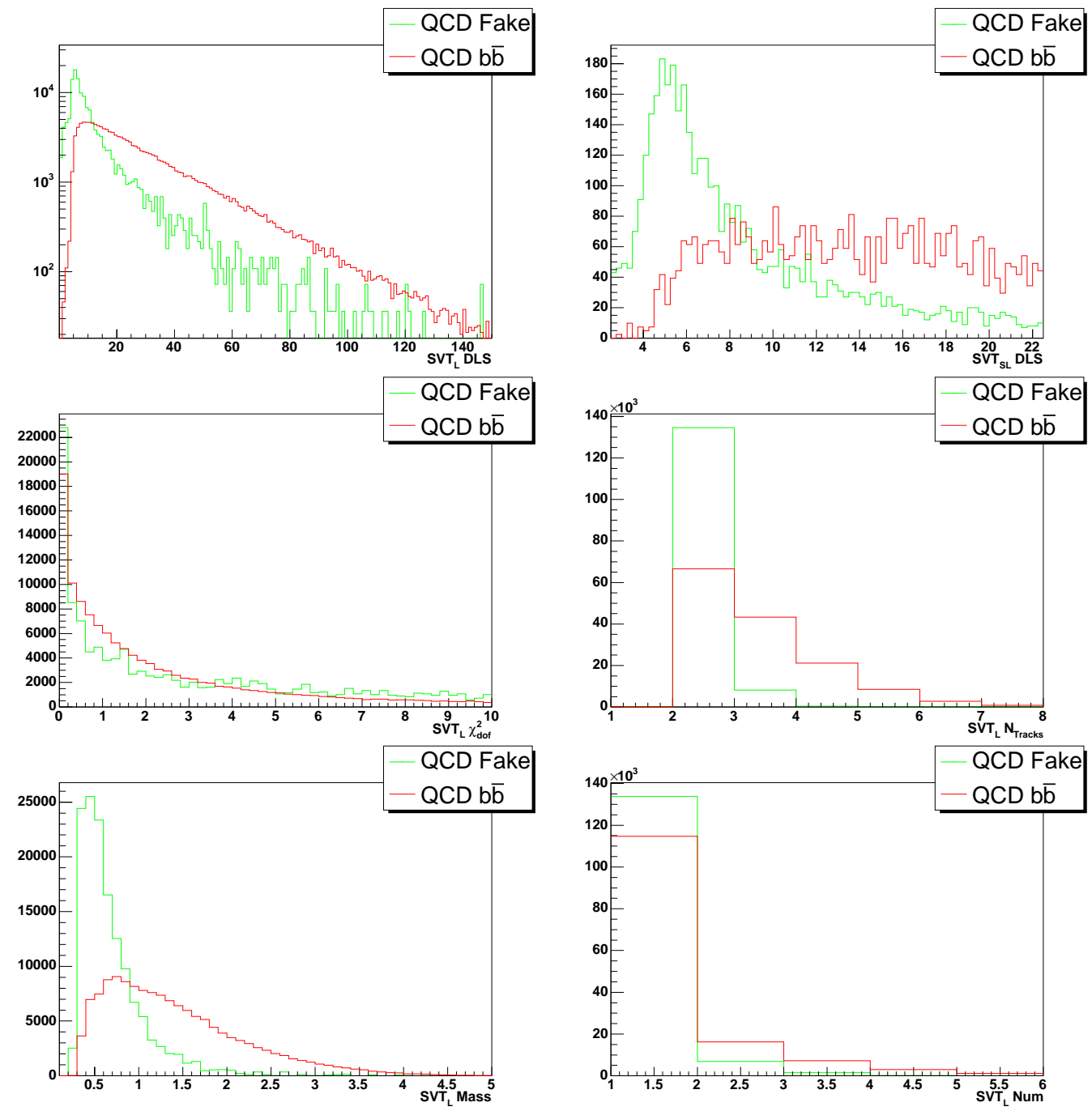

Figure 4.3: Variables for the Loose SVT tagger, DLS (top left and right), $\chi_{\text {dof }}^{2}$ (middle left), $N_{\text {Tracks }}$ (middle right), Mass (bottom left) and number of vertices (bottom right) for QCD $b \bar{b}$ (red) and fake (green) MC jets. See the text for a full description of the variables. 
information, and therefore it is better to have information on all possible SVs in an event, even if they are poorly reconstructed.

To increase the amount of information available, a 'Super Loose' version of the SVT tagger was defined $\left(\mathrm{SVT}_{S L}\right)$. The $\mathrm{SVT}_{S L}$ has a looser track selection criteria than that used by the standard SVT tagger operating points. The track selection used for the standard SVT tagger operating points and those used for the $\mathrm{SVT}_{S L}$ are shown in Table 4.6. Figure 4.4 shows the efficiency and fake rate of the $\mathrm{SVT}_{L}$ and $\mathrm{SVT}_{S L}$ taggers on QCD $b$ and fake-jet samples. The $\mathrm{SVT}_{S L}$ tagger provides information on $\sim 90 \%$ of the $b$-jets compared to $\sim 65 \%$ for the $\mathrm{SVT}_{L}$ tagger. The larger number of both fake and $b$-jets is advantageous as it provides a larger training sample.

\begin{tabular}{|c|c|c|c|c|}
\hline Track Cuts & Tight & Medium & Loose & Super Loose \\
\hline$\chi^{2}$ & 3 & 10 & 10 & 15 \\
$I P_{\text {sig }}$ & 3.5 & 3.5 & 3 & 0.0 \\
$\mathrm{p}_{T}$ GeV & 1.0 & 1.0 & 1.0 & 0.5 \\
Num SMT Hits & 2 & 2 & 2 & 2 \\
\hline
\end{tabular}

Table 4.6: The track selection cuts used for the standard SVT operating points and the Super Loose SVT tagger. The tracks are selected based upon their $\chi^{2}$, impact parameter significance $\left(I P_{\text {sig }}\right)$, transverse momentum $\left(\mathrm{p}_{T}\right)$ and number of SMT hits.
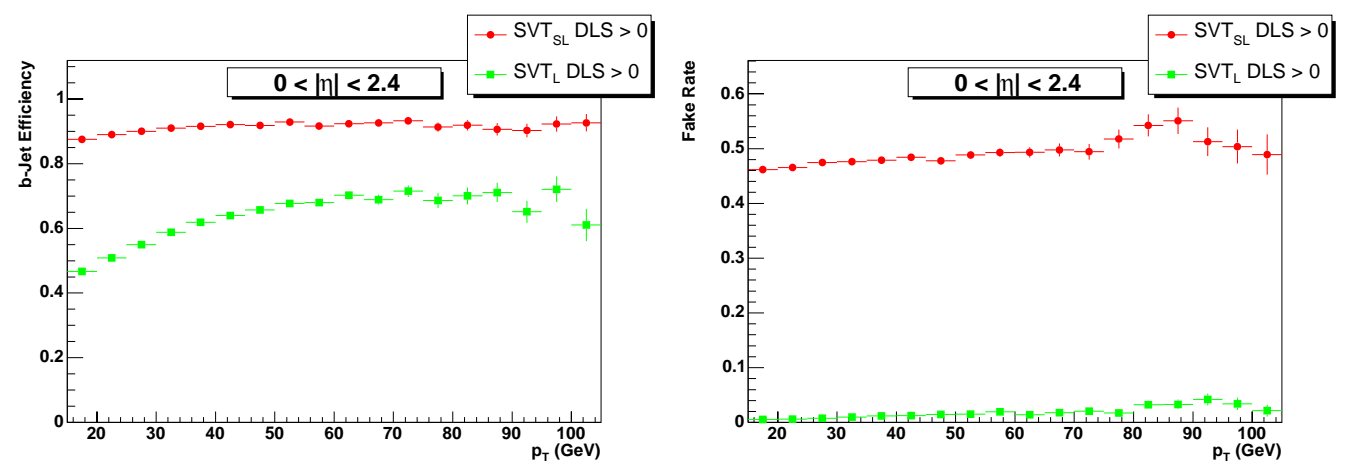

Figure 4.4: $\mathrm{SVT}_{S L}$ (red circles) and $\mathrm{SVT}_{L}$ (green squares) efficiencies for QCD $b \bar{b}$ and QCD fake MC jets.

\subsubsection{CSIP Variables}

Potential variables from the Loose CSIP tagger [48] are listed below and shown in Fig. 4.5. 
CSIP $3 s$ - The number of tracks with a decay length significance greater than 3 .

CSIP $2 s$ - The number of tracks with a decay length significance greater than 2 .

CSIP $3 w$ - The number of tracks with a negative decay length significance greater than 3 and an angle to the jet axis $<1.15$ radians.

CSIP $2 w$ - The number of tracks with a negative decay length significance greater than 2 and an angle to the jet axis $<1.15$ radians.

All the CSIP variables are small integer values which are not ideal inputs for a NN. NNs perform best when provided with continuous values spread over a range. Therefore, the standard CSIP variables were combined into a single variable, spread over a greater range. Replacing four variables with one also had the advantage of reducing the number of input variables and so simplifying the NN. The weights were determined in an entirely empirical manner to give optimum performance:

$$
\text { CSIP Comb }=6 \times 3 s+4 \times 2 s+3 \times 3 w+2 \times 2 w
$$

Using the variables as separate inputs was also investigated.

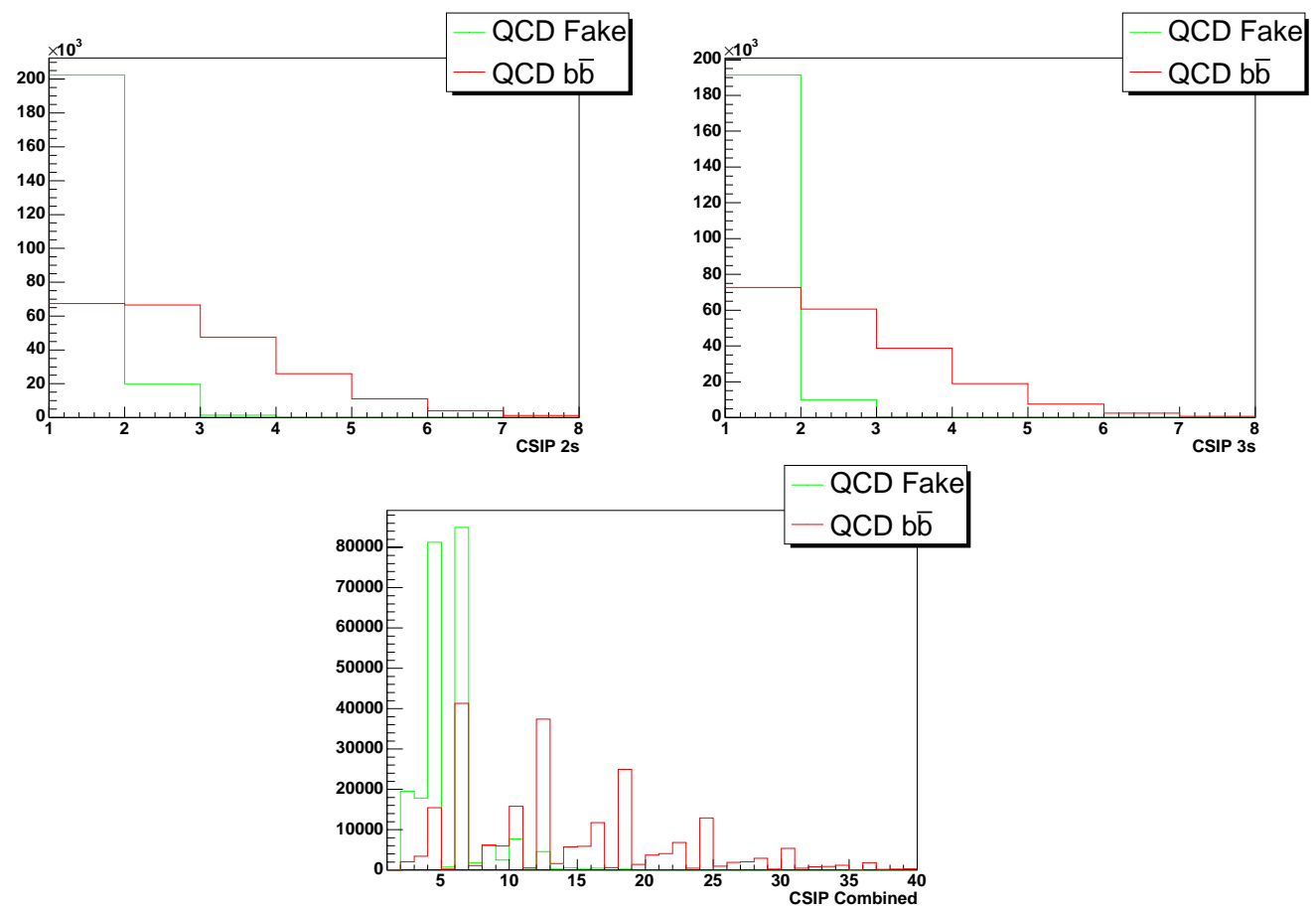

Figure 4.5: Variables from the Loose CSIP tagger, the number of tracks with a significance $\geq 2$ (top left), the number of tracks with a significance $\geq 3$ (top right) and the combination variable (bottom) for QCD $b \bar{b}$ (red) and fake (green) MC jets. See the text for a full description of the variables. 


\subsubsection{SLT}

No input from the Soft Lepton Tag (SLT) tagger [51] was used in the NN. The reasons for this are twofold. Firstly, the SLT is used in the 'System8' efficiency calculation to measure a tagger's efficiency on data [86]. The System8 measurement requires two taggers which are uncorrelated and using the SLT as a NN input would correlate the output of the two taggers. The SLT tagger has been shown to be uncorrelated with the JLIP [76], SVT [77] and CSIP [48] taggers. Secondly, from a performance perspective, the SLT returns no output for the majority of events and a variable which is zero for the majority of events has very little benefit. Tests showed that the addition of SLT variables on an inclusive jet sample did not improve the performance.

\subsubsection{Primary Vertex}

The quality of the primary vertex $(\mathrm{PV})$ is a very important indicator of the tracking quality in an event. However, the PV variables are not used in the NN due to possible, currently unstudied, correlations that may arise between jets when using a global event variable. However, if these correlations can be shown to be small, then these variables could be included in future versions of the NN tagger. Tests showed this to be a powerful variable.

\subsubsection{Jet Variables}

In order to construct a generic tagger, which is independent of any particular jet reconstruction algorithm, jet algorithm-dependent variables like jet $\mathrm{p}_{T}$ are not used.

\subsection{Neural Network Optimisation}

Six attributes need studying to produce an optimised NN: the input variables (number and type), the input selection criteria (the selection of jets to be separated by the $\mathrm{NN}$ ), the structure, the training algorithm, the training sample and the number of training epochs. In addition, a benchmark with which to measure the performance needs to be defined.

The most important attribute which needs optimising is the input variables. The input variables were optimised by selecting interim values for the other attributes whose optimisation would depend upon the final choice of input variables. Initial values, which were later re-optimised, were set for the structure $(\mathrm{N}: 2 \mathrm{~N}: 1$, where $\mathrm{N}$ is the number of input variables), number of training epochs (500) and the input jet 
cuts (SVT DLS $>2$ or CSIP COMB $>8$ or JLIP Prob $<0.02$ ). The training samples and training algorithms were optimised prior to the input variable optimisation as they did not depend on the input variable optimisation.

\subsubsection{Training Samples}

Trees of 270,000 signal (QCD $b \bar{b}$ ) and 470,000 background (QCD fake) jets, weighted to be of equal number (after input selection cuts), were used in the training and testing of the NN. The signal and background samples were split in half, with half used for the test sample and the other half for the training sample. Figure 4.6 shows the $\mathrm{p}_{T}$ and $\eta$ of the training and testing sample. Signal and background training samples have approximately equal $\mathrm{p}_{T}$ and $\eta$ spectra to avoid any $\mathrm{p}_{T}$ and $\eta$ biases that may be caused by using different spectrums for the signal and background.
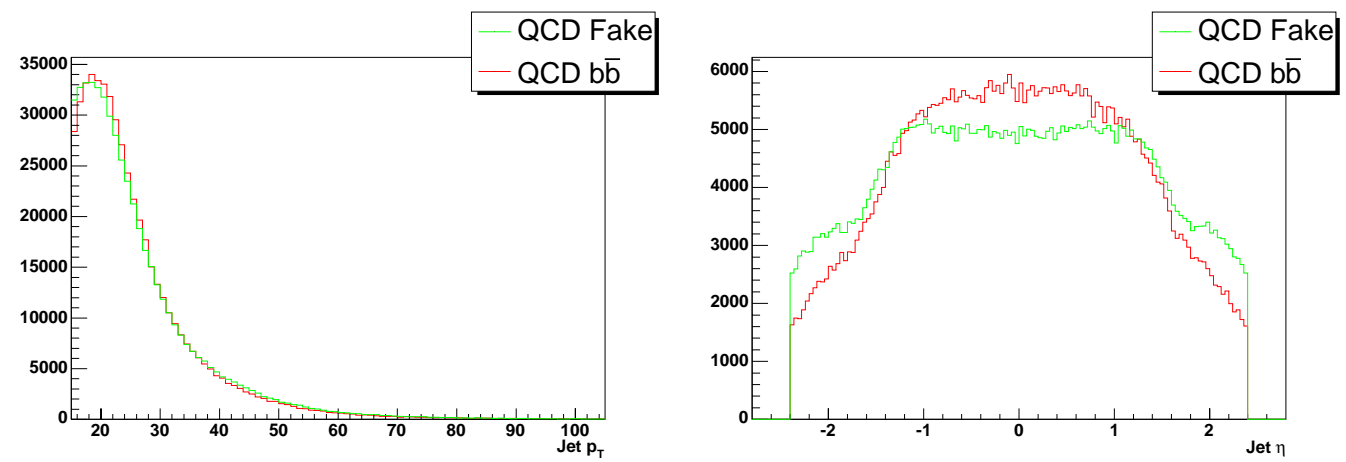

Figure 4.6: The $\mathrm{p}_{T}$ (left) and the $\eta$ (right) of the training and test samples.

\subsubsection{Training Algorithms}

Figure 4.7 shows the comparative training curves for six different training algorithms for a simple NN consisting of JLIP Prob, SVT DLS, CSIP Comb and SVT $\chi_{d o f}^{2}$ for a fixed amount of CPU processing time. The BFGS training algorithm is the most consistent and systematic method to minimise the error on the fit, and was therefore used as the minimisation algorithm.

\subsubsection{Performance Benchmark}

Each of the attributes in the following sections were optimised according to the fake rate achieved for a fixed $b$-efficiency (the lower the fake rate the better the performance). The performance of the $\mathrm{NN}$ was measured by comparing the fake rates at four fixed $b$-efficiencies $75 \%, 70 \%, 60 \%$ and $50 \%$. 


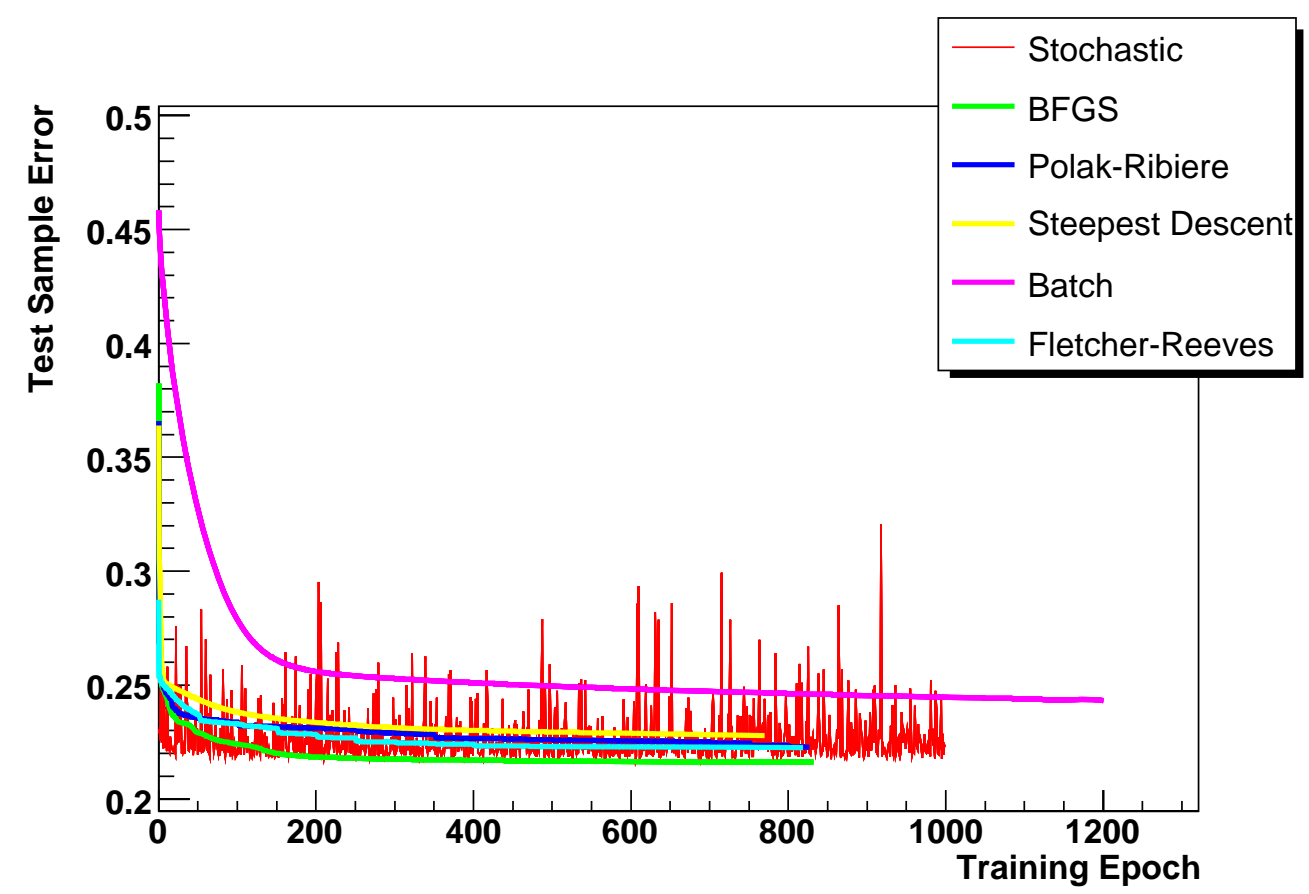

Figure 4.7: Training curves for the various training algorithms for a fixed amount of CPU time.

All the training was carried out on the QCD samples, and the performance measurements were carried out on the Alpgen [85] tz MC. The performance measurements were also cross checked on the merged Pythia [70] QCD MC samples (see Section 4.1.3 for a description of the different samples).

\subsubsection{Variable Optimisation}

The most time-consuming part in constructing a NN is selecting the input variables. The greater the number of variables, the greater the amount of information, and therefore the better the discrimination, but to reduce systematic uncertainties, it is desirable to have the minimum number of variables possible. Another problem is the selection of which variables to use together. Although variables on their own might not have much effect, in combination with another variable a powerful pattern could emerge.

It would be very time consuming to test all the possible combinations of variables. If all the different possible $\mathrm{NNs}$ for the 14 variables identified above were tested, it would take the construction of 16,368 different networks to identify the optimal solution. This is obviously impractical. One solution to this problem is to individually identify the most powerful variables. If we have an initial NN with $n$ variables and a list of $m$ variables we want to rank in order of power, the following procedure can be carried out to identify the most powerful variables: 
1. Individually add each of the $m$ variables to be tested to the initial $n$ variable NN so we have $m$ NNs each with $n+1$ variables.

2. Identify the variable whose addition improved the NN's performance the most, and add this variable permanently to the $\mathrm{n}$ variable $\mathrm{NN}$.

3. Go to step 1) and test each of the remaining $m-1$ variables with the new $n+1$ variable NN.

Although this method has its limitations, such as possibly missing an optimal combination of variables that did not have any discrimination when added separately, it is an effective way of systematically identifying the best variables and the optimal number to use for a specific operating point.

To identify the most powerful variables the above procedure was followed, starting with an initial two variable $\mathrm{NN}$ which was identified by testing every possible combination of variables in a two input NN. The remaining variables were then ranked in order of power by examining the fake rate at the $70 \%$-efficiency operating point.

As a cross check the procedure was repeated for signal efficiencies of 50,60 and $70 \%$. The same set of variables was found to give the greatest reduction in fake rate in each case. Two extreme efficiencies are shown by way of example in Fig. 4.8 and the variables are listed in order of power in Table 4.7 .

\begin{tabular}{|c|c|}
\hline Rank & Variable \\
\hline 1 & SVT $_{S L}$ DLS \\
1 & CSIP Comb $^{-}$ \\
\hline 3 & JLIP Prob \\
4 & SVT $_{S L} \chi_{\text {dof }}^{2}$ \\
5 & SVT $_{L} N_{\text {Tracks }}$ \\
6 & SVT $_{S L}$ Mass \\
7 & SVT $_{S L}$ Num \\
8 & JLIP Prob $_{\text {red }}$ \\
9 & SVT $_{S L}$ dR \\
\hline
\end{tabular}

Table 4.7: NN input variables ranked in order of power.

The NNs with 5, 7 and 9 variables were identified for further study, and Fig. 4.9 shows the performance when using 5, 7 and 9 variables. The NN with 5 variables 

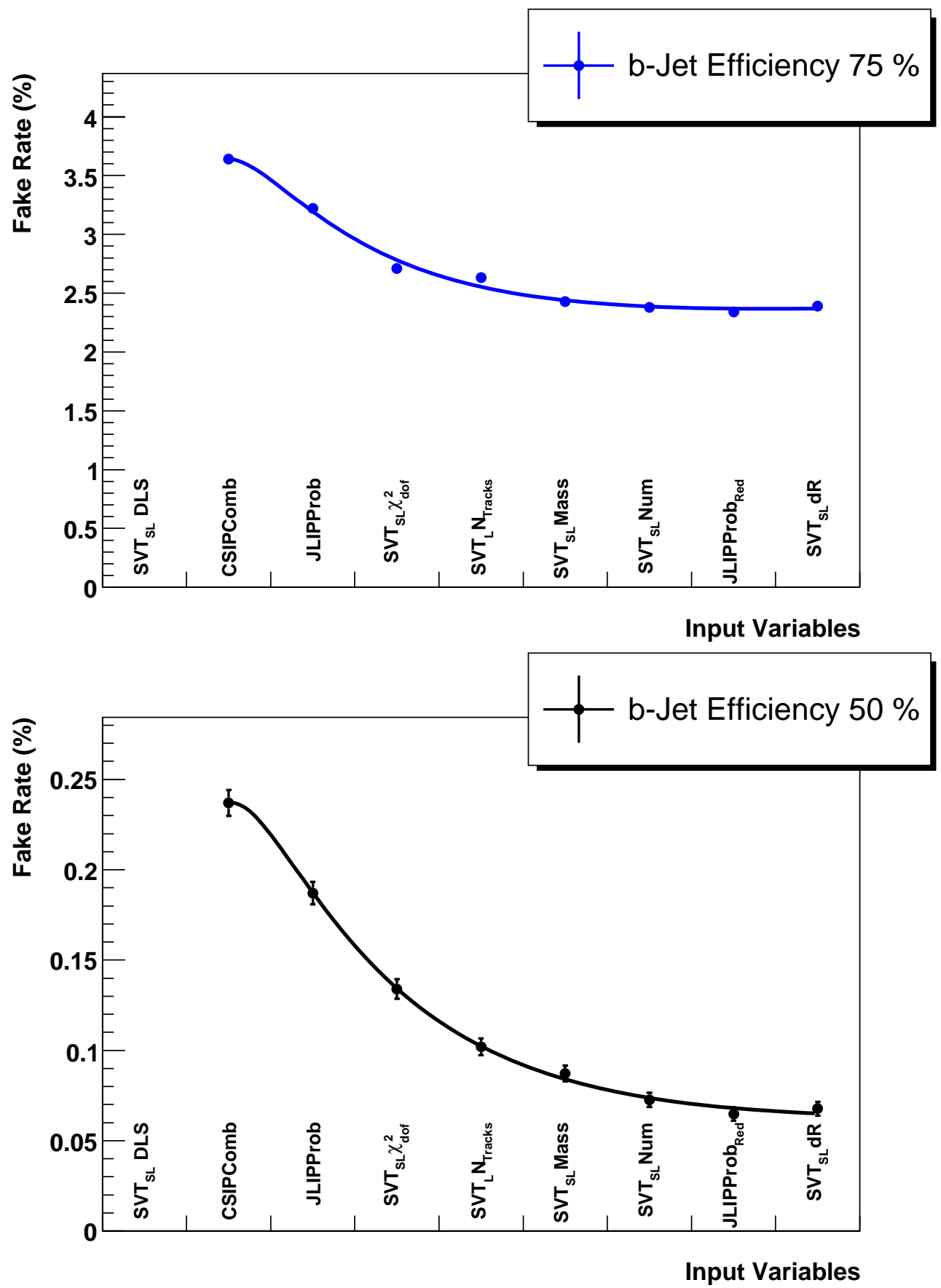

Figure 4.8: Fake rate for fixed signal efficiencies of $75 \%$ (top), and $50 \%$ (bottom) as a function of additional NN variables. The NN variables were added to the NN in order of performance. The fit is intended to guide the eye only. The errors are statistical only. 
benefits from the majority of the improvements in performance and has the advantage of being the simplest. However, the NNs with 7 and 9 variables have a better performance, but at the price of an increased number of variables. When going from 7 to 9 variables there is very little improvement. Therefore the 7 variable NN is the optimal solution. The final selected variables were SVT $_{S L}$ DLS, CSIP Comb, JLIP Prob, $\mathrm{SVT}_{S L} \chi_{\text {dof }}^{2}, \mathrm{SVT}_{L} N_{\text {Tracks }}, \mathrm{SVT}_{S L}$ Mass and $\mathrm{SVT}_{S L}$ Num.

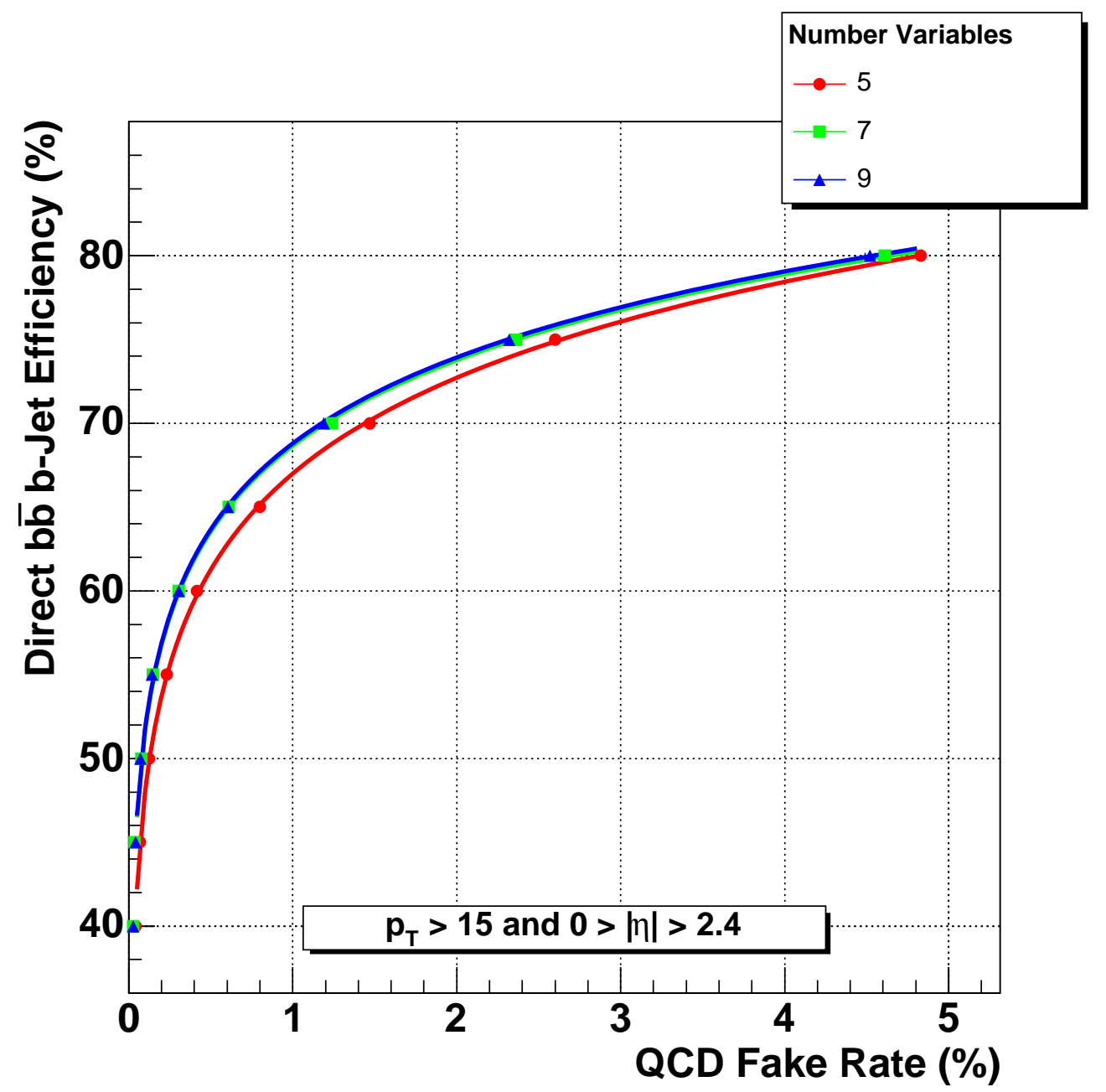

Figure 4.9: Performance curves for the 5 (red circles), 7 (green squares) and 9 (blue triangles) variable NN. The 7 and 9 variable NNs have better performance than the 5 variable NN, although there is no improvement in going from 7 to 9 variables.

\subsubsection{Number of Training Epochs}

This was varied from 50 up to 2000 training epochs, with the results presented in Fig. 4.10. For each of the operating points the majority of the minimisation is reached by $\sim 200$ epochs, with only additional small improvement thereafter. To ensure optimised NNs the number of training epochs was set to 1000. 

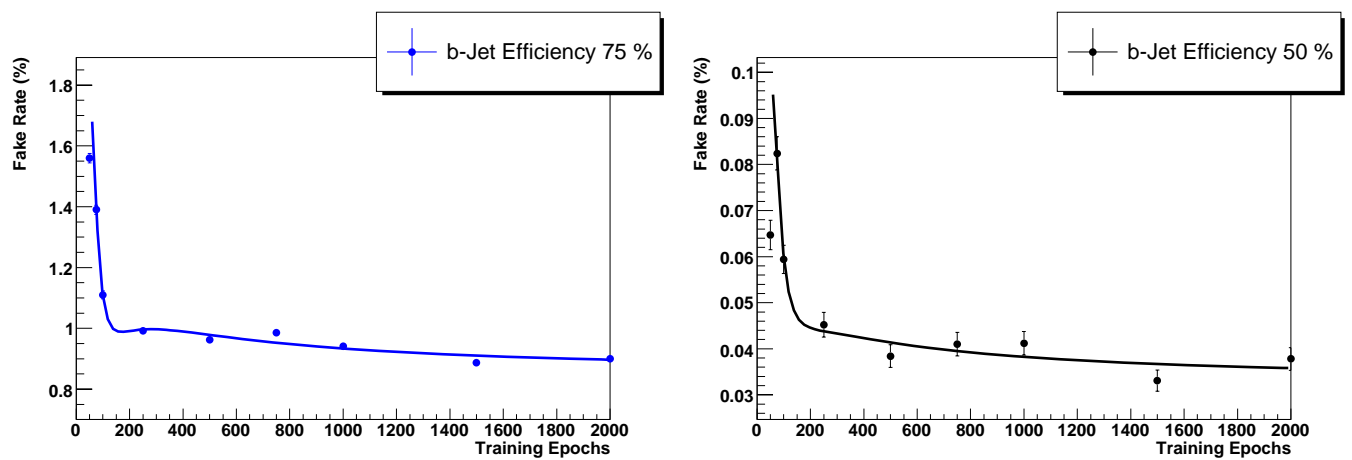

Figure 4.10: Fake rate for fixed signal efficiencies of $75 \%$ (left) and $50 \%$ (right) as a function of the number of training epochs. The majority of the minimisation is achieved by 200 epochs for each of the operating points with only small increases in performance thereafter. The fit is intended to guide the eye only.

\subsubsection{Neural Network Structure}

During the optimisation of the input variables, the number of hidden nodes was set to $2 \mathrm{~N}$, where $\mathrm{N}$ is the number of input nodes. The number of hidden nodes was optimised by varying their number from 7 through 34 . The results are shown in Fig. 4.11. 24 is the optimal number of hidden nodes for each operating point.
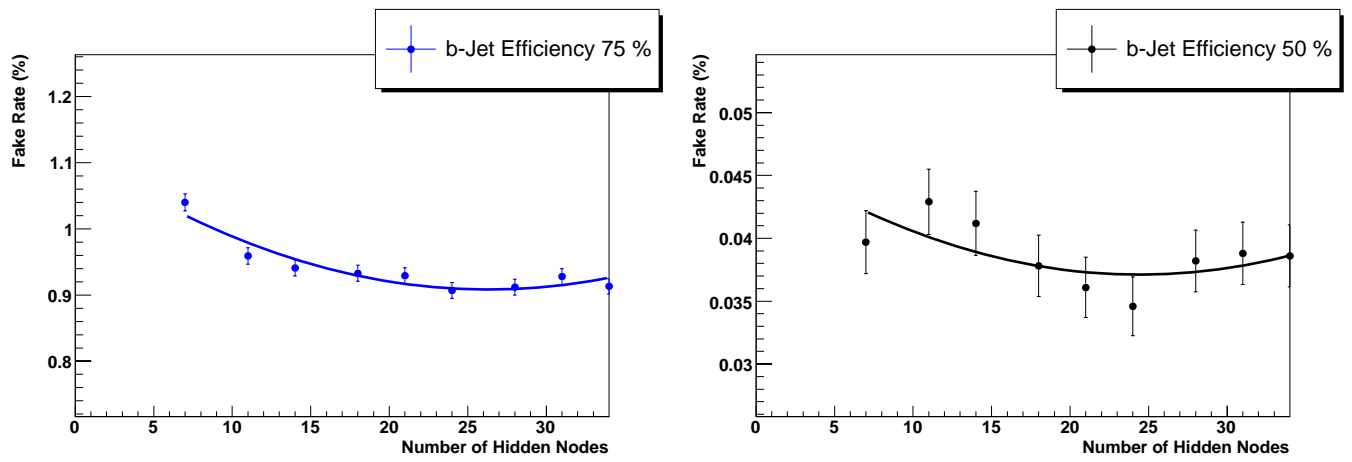

Figure 4.11: Fake rate for fixed signal efficiencies of $75 \%$ (left) and $50 \%$ (right) as a function of the number of hidden nodes in a single hidden layer. There is a minimum at 24 hidden nodes for each of the operating points. The fits are designed to guide the eye only.

The NN output using the structure 7:24:1, is shown in Fig. 4.12 (left). The single hidden layer NN does not produce an output normalised between 0 and 1 . However, it should be possible to replicate any continuous function with only one hidden layer. This situation occurs as a NN will provide only an approximate fit to a function. The fit may not therefore necessarily be constrained between 0 and 1 unless the output node is set to normalise the output. In the TMultiLayerPerceptron implementation, the output node is not a sigmoid function ${ }^{4}$, and consequently does

\footnotetext{
${ }^{4}$ In the latest versions of the TMultiLayerPerceptron code the output node is now capable of normalising the output. However, when this work was undertaken this was not the case.
} 
not normalise the output from the single layer to be between 0 and 1 .

From this reasoning it follows that by adding an extra hidden layer with 1 node the output will be correctly normalised. The extra sigmoid should not alter the performance of the NN, but should normalise the output to ensure that it is between 0 and 1 . Figure 4.12 (right) shows the output of such a NN, which retains the same shape in the regions $>0$ and $<1$, but with all the outlying points collected into the peaks at 0 and 1 . This NN had a marginally improved performance when compared to the single layer NN.

This happens as the single hidden layer of 24 nodes achieves the optimal fit, which the extra layer then normalises between 0 and 1 . This was corroborated by adding 12 nodes into the second hidden layer and verifying that neither the performance or shape of the output was altered by the additional nodes.
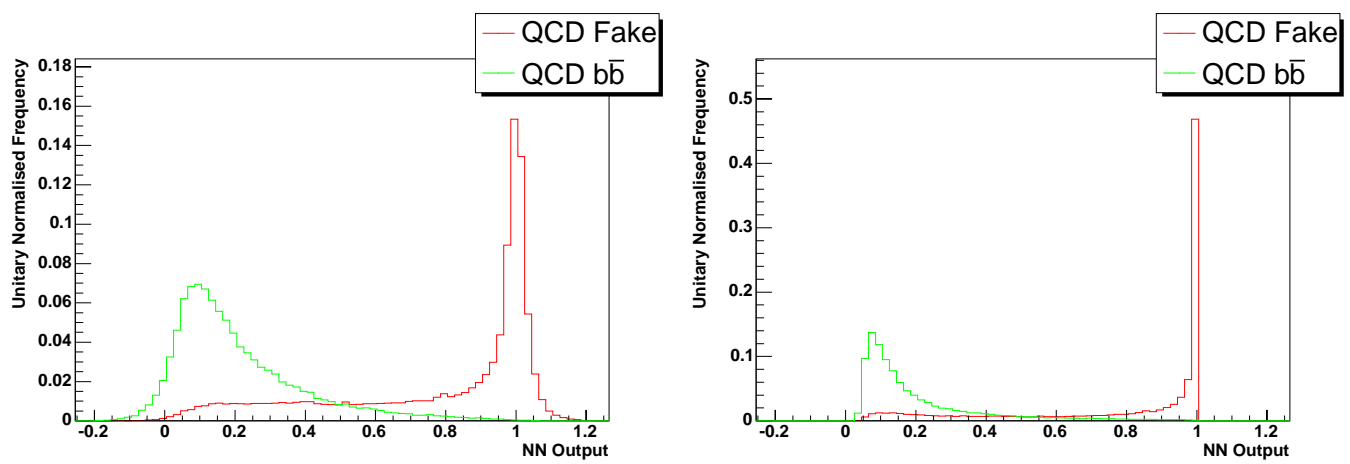

Figure 4.12: The NN output for MC QCD $b \bar{b}$ (red) and fake-jets (green) for the case of a single hidden layer 7:24:1 (left) and double hidden layer 7:24:1:1 (right). The extra hidden layer constrains the $\mathrm{NN}$ between 0 and 1 .

\subsubsection{Input Selection Cuts}

One of the most important aspects of the $\mathrm{NN}$ is the selection of the jets which will be input into the NN. Too loose a selection will mean a loss of performance and resolution as the $\mathrm{NN}$ is separating signal from background which could have been carried out with a simple cut. However, too stringent a selection will cause a significant loss of $b$-jets and therefore limit the NN's performance at high efficiencies. Cuts are necessary so that jets have information for at least one of the input variables.

The input selection cuts were optimised in order of the input tagger's input tagging efficiency (the efficiency of an input tagger to have a non-zero tagging result for a jet). The variables were optimised in the order $\mathrm{SVT}_{s l} \mathrm{DLS}$, JLIP Prob and CSIP Comb. 
The $\mathrm{SVT}_{S L}$ DLS is the most important variable as small variations in its value can make large differences to the number of $b$ and fake-jets inputted to the NN. The $\mathrm{SVT}_{S L}$ DLS cut was varied from 0.5 to 4.0, with the results shown in Fig. 4.13 (top). A cut value of 2.5 benefits from the vast majority of the gains in tightening this cut, whilst being slightly conservative.

The JLIP Prob cut was altered from 0.005 to 0.4 , with the results shown in Fig. 4.13 (middle). A cut value of 0.02 substantially lowers the fake rate at the $50 \%$ and $60 \%$ operating points whilst having a negligible effect on the higher efficiency operating points.

The CSIP Comb cut was varied between 2 and 14 as shown in Fig. 4.13 (bottom). Varying the cut value does not have a significant effect on the fake rate and deviations between different cut values are mostly within errors. A cut value of 8 does however consistently appear to minimise the fake rate for the majority of operating points.

\subsubsection{Optimised NN Parameters}

The optimised parameter values for the NN tagger are shown in Table 4.8.

\begin{tabular}{|c|c|}
\hline Parameter & Value \\
\hline NN structure & $7: 24: 1: 1$ \\
\hline $\begin{array}{l}\text { Input Variables } \\
\text { (Ranked) }\end{array}$ & $\begin{array}{l}\text { (1) } \mathrm{SVT}_{S L} \text { DLS (2) CSIP Comb } \\
\text { (3) } \mathrm{JLIP} \text { Prob (4) } \mathrm{SVT}_{S L} \chi_{\text {dof }}^{2} \\
\text { (5) } \mathrm{SVT}_{S L} N_{\text {Num }} \text { (6) } \mathrm{SVT}_{L} N_{\text {Tracks }} \\
\text { (7) } \mathrm{SVT}_{S L} \mathrm{Num}\end{array}$ \\
\hline $\begin{array}{l}\text { Jet input selection cuts } \\
\text { (failure results in NN output of } 0 \text { ) }\end{array}$ & $\begin{array}{c}\text { SVT }_{S L} \text { DLS }>2.5 \\
\text { or JLIP Prob }<0.02 \\
\text { or CSIP Comb }>8\end{array}$ \\
\hline Number of training epochs & 1000 \\
\hline $\begin{array}{l}\text { Number of } b \text {-jets used in training } \\
\text { (after jet selection cuts) }\end{array}$ & $\begin{array}{c}135,000 \\
(110,000)\end{array}$ \\
\hline $\begin{array}{l}\text { Number of fake-jets used in training } \\
\text { (after jet selection cuts) }\end{array}$ & $\begin{array}{l}235,000 \\
(20,000)\end{array}$ \\
\hline
\end{tabular}

Table 4.8: Optimised NN parameters. 

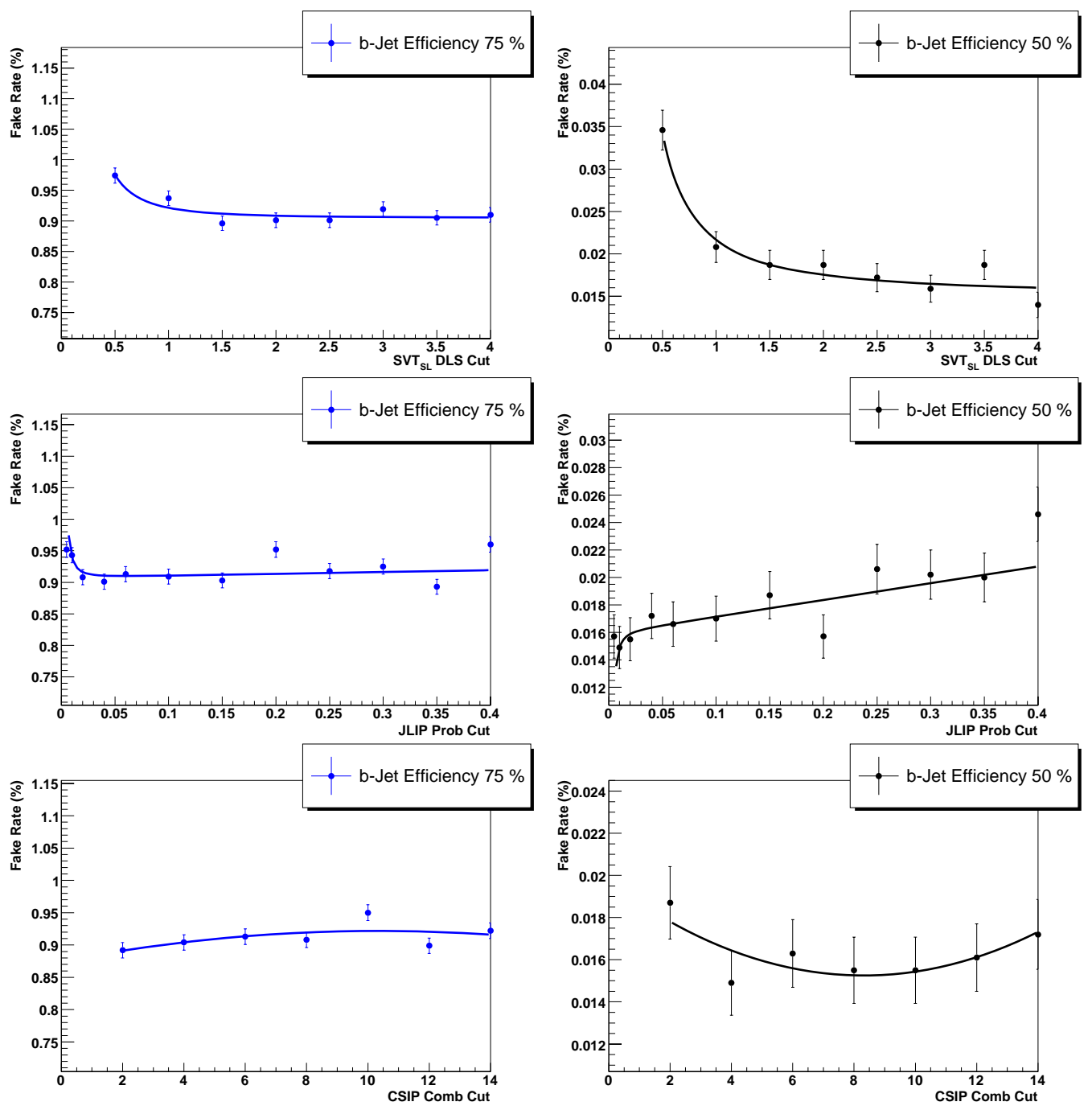

Figure 4.13: Fake rate for fixed signal efficiencies of $75 \%$ (left) and $50 \%$ (right) as a function of the $\mathrm{SVT}_{S L}$ DLS cut (top), JLIP Prob cut (middle) and CSIP Comb cut (bottom) on the input jets. The fits are designed to guide the eye only. 


\subsection{Monte Carlo Performance}

The NN output for the optimised NN b-tagger on the signal and background training samples is shown in Fig. 4.14. The NN achieves a significant separation between the $b$-jets and $b$-like fake-jets. A $b$-like fake-jet is one which has passed the input selection cuts for the NN tagger, i.e. it has (a loosely defined) b-tag.

The performance of the NN tagger compared to the JLIP tagger is shown in Fig. 4.15. There is a substantial improvement over the JLIP tagger, with relative increases in the efficiency of $\sim 50 \%$ for a fake rate of $0.2 \%$, and $\sim 15 \%$ for a fake rate of $4.0 \%$. The fake rate is reduced by a factor of five for fixed signal efficiencies.

The performance of the NN tagger compared to the SVT, CSIP and JLIP taggers is shown in Fig. 4.16 as a function of $\mathrm{p}_{T}$ and $\eta$. The Loose versions of the SVT and CSIP taggers are used and the JLIP tagger is set to JLIP Prob $<0.006$ to ensure similar fake rates. The NN tagger has a significantly better efficiency compared to the individual input $b$-tagging tools over all $\eta$ and $\mathrm{p}_{T}$, although this improvement falls off at high $\mathrm{p}_{T}$. At the high $\mathrm{p}_{T}$ values, the $\mathrm{NN}$ tagger has a substantially lower fake rate than the SVT and CSIP taggers. At high $\eta$ values the fake rate of the NN tagger appears to be higher than the constituent input $b$-taggers, although there are low statistics in this region.

\subsection{Data and Monte Carlo Variable Comparison}

As the $\mathrm{NN}$ is trained on MC events, but is to be applied to both data and MC events, the input variables were compared in $\mathrm{MC}$ and data to ensure good agreement. This comparison was carried out in two stages. Firstly, the input variables were compared on an individual basis for both fake and $b$-jets to ensure that there are no major disagreements. Secondly, to check the correlations between input variables, the NN output was compared between the data and MC $b$ and fake-jet samples. Any problems caused by different correlations between the variables would have been evident in the NN output.

\subsection{1 b-Jet Input Variables}

A $b$-enriched jet data sample was obtained from the muonic jets in the $b$-ID skim. The $b$-content of the sample was enhanced, without biasing the variable comparison, by applying SLT and an away tag (see Section 4.1.1.3). 


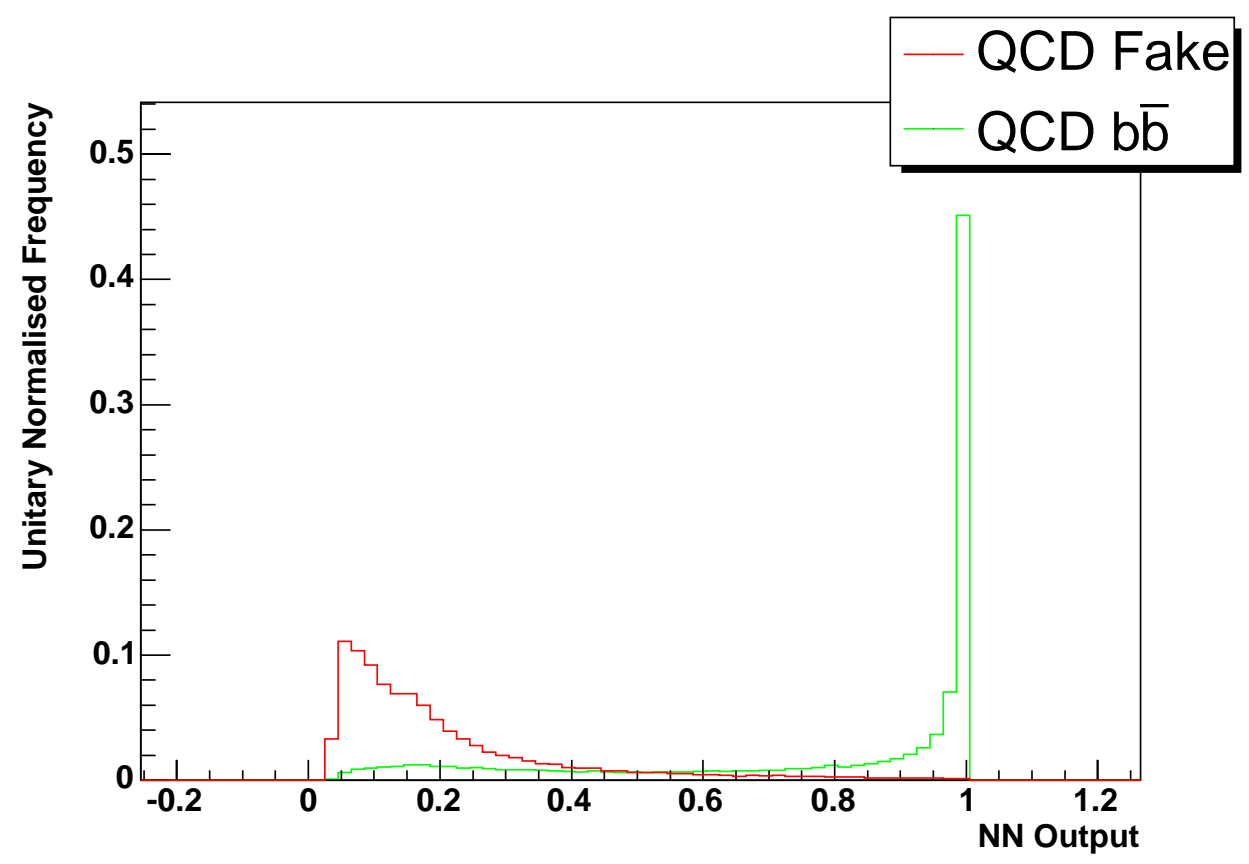

Figure 4.14: NN output for QCD MC $b$ (green) and fake-jet (red) samples, after the NN jet input selection cuts.

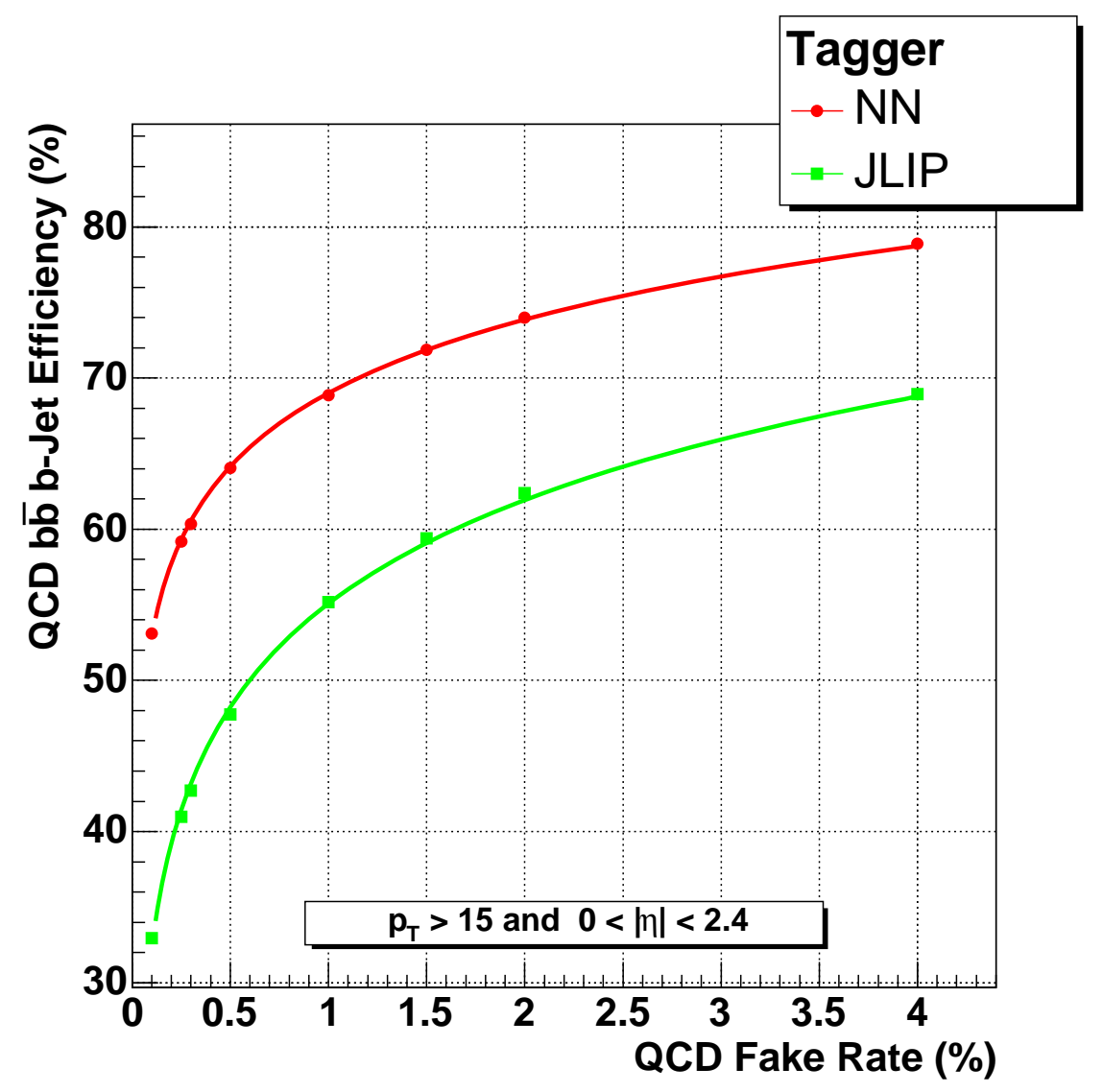

Figure 4.15: Performance curves for the NN tagger (red circles) and the JLIP tagger (green squares) for QCD $b$ and $b$-like fake-jet samples. 

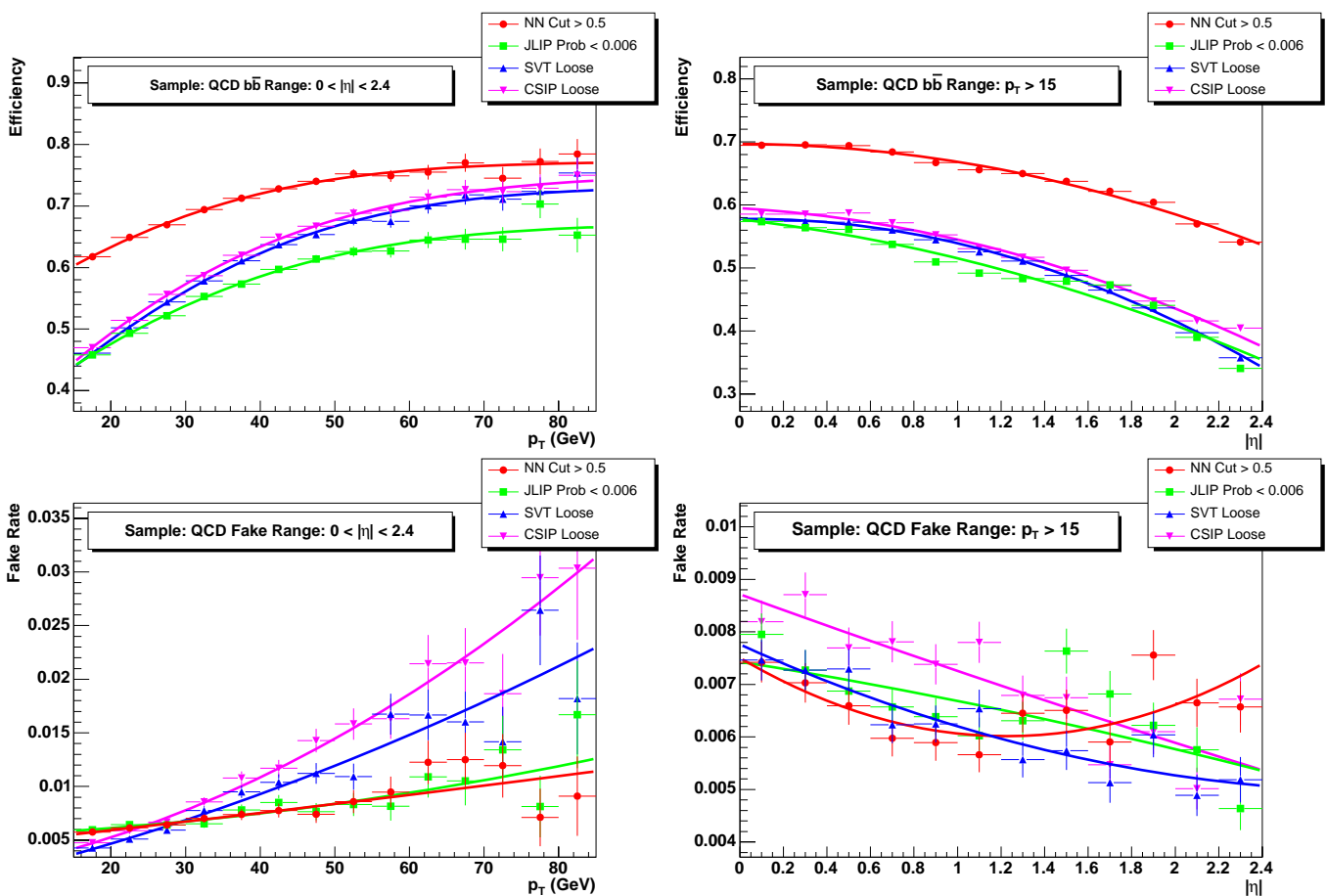

Figure 4.16: Tag rates for the JLIP (green squares), CSIP (purple triangles), SVT (blue triangles) and NN (red circles) taggers on QCD $b$ (top) and fake-jet (bottom) samples in $\mathrm{p}_{T}$ (left) and $\eta$ (right) projections.

The SLT tag was defined as a muon with a $p_{\text {TRel }}>0.7 \mathrm{GeV}^{5}$. Applying a SLT tag will not bias the variable comparison as the SLT tagger has been shown to be uncorrelated with the other taggers (see Section 4.2.4). The away tag used the cuts outlined in Table 4.9 which were designed by examining $b, c$ and fake-jet tagging variables in MC and selecting values which maximised the $b$-efficiency relative to the $c$-efficiency. The efficiency of the away tag was estimated from QCD MC. Events were selected which had exactly two taggable jets, and the probability that the fake, $c$ and $b$-jets present in the events had an away tag was measured. This measurement takes into account the effects of possible gluon splitting to collinear heavy flavour in the away jet and QCD production of jets of different flavours. Although these efficiencies are measured entirely on MC, they should prove sufficient as a rough estimate of the flavour content of the $b$-enriched sample. The away tag efficiencies along with the SLT tagging efficiencies and the estimated flavour content of the $b$-enriched sample are given in Table 4.10. The fact that the overall $b$-efficiency is very small is not a problem due to the large data sample available.

The $b$-enriched data sample is compared to $b$-jets from a QCD MC sample. All jets have passed the NN jet input selection cuts. The input variables from both samples are shown in Fig. 4.17.

\footnotetext{
${ }^{5} p_{T R e l}$ is the $\mathrm{p}_{T}$ of the muon relative to the combined jet and muon $\mathrm{p}_{T}$ axis.
} 


\begin{tabular}{|c|c|}
\hline Variable & Cut Value \\
\hline JLIP Prob & $<0.002$ \\
JLIP Prob Red & $<0.02$ \\
Tight SVT DLS & $>10$ \\
CSIP 3s & $>2$ \\
CSIP Mass & $>3.0$ \\
\hline
\end{tabular}

Table 4.9: Away tag cut applied to the $b$-ID skim. Designed to primarily reduce the $c$-jet content in the $b$-enriched sample.

\begin{tabular}{|c|c|c|c|c|}
\hline Flavour & QCD Content & SLT Eff & Away Tag Eff & Content \\
\hline$b$ & $3 \%$ & $23.0 \%$ & $2.40 \%$ & $96.5 \%$ \\
$c$ & $5 \%$ & $8.4 \%$ & $0.25 \%$ & $3.2 \%$ \\
Fake & $92 \%$ & $0.05 \%$ & $0.24 \%$ & $0.3 \%$ \\
\hline
\end{tabular}

Table 4.10: The QCD content (estimated from QCD MC) along with the SLT and away tag efficiencies used to estimate the flavour content in the $b$-enriched sample.

The data $b$-jets have less $b$-like tagging results than those in MC. This is expected due to the MC's over-optimistic tagging results and the $u d s c g$ contamination in the data sample which degrades the measured data tagging results. Although the MC and data do not agree exactly, there is no major disagreement, and enough agreement between the variables to justify training on $\mathrm{MC}$ for application to data, subject to further crosschecks in the following sections.

\subsubsection{Fake-Jet Input Variables}

Obtaining a sample of fake-jets without any, or with minimal heavy flavour contamination is challenging. Any cuts placed on the jet will bias the input variable comparison and little would be gained by using an away tag method to enrich a sample with fake-jets. However, by using NT results (see section 4.1.1.2) instead of PT results, a good approximation to a sample of fake-jets can be achieved. Heavy flavour fractions of approximately $5 \% c$ and $3 \% b$ were measured using QCD MC. When the jet input selection cuts are applied the fraction of heavy jets in the sample increases to $\sim 10 \% c$ and $\sim 10 \% b$. However, as the NT results are being used, any $b$-like contribution from the heavy flavour component will be heavily suppressed.

The NT data sample is compared to the NT results from the MC QCD sample and is shown in Fig. 4.18. There is generally good agreement between the fake-jet 
variables in MC and data, with the main differences existing between the low JLIP Prob values. The disagreement between data and MC in this region is likely caused by the presence of $b$ and $c$-jets in the NT samples which are responsible for the majority of results at low JLIP Prob values. The over optimistic tagging results for MC $b$ and $c$-jets will produce a discrepancy between the data and MC samples in this region.

\subsubsection{Data and Monte Carlo Neural Network Output Com- parison}

The NN outputs for the EM and QCD and the $b$-enriched samples compared to their corresponding MC samples are shown in Fig. 4.19.

There is good agreement between data and MC with any differences explained by the discrepancies in the input variable comparisons. The data $b$-jet NN output is more peaked due to a combination of contamination from $u d s c g$-jets and the lower tagging efficiency in data. The fake-jets show very good agreement as would be expected from the good agreement between the data and MC input variables. The good agreement in these plots suggests that the input variables and their correlations are similar between data and MC.

\section{6 b-Efficiency Measurement}

The efficiency of the NN tagger on data is measured in the following section for inclusive $b$ and $c$-jets; the statistical and systematic error associated with the measurement are also derived.

The process by which the TRF for inclusive $b$ and $c$-jets for data are measured is illustrated in Fig. 4.20, is briefly outlined below, and is explained in more detail in the rest of this section:

1. A $b$-jet TRF is measured for data using muonic $b$-jets ${ }^{6}$. This muonic $b$-jet TRF is not however equivalent to the inclusive $b$-jet $\operatorname{TRF}^{7}[77]$.

2. A muonic $b$-jet TRF is measured for MC.

3. A data/MC scale factor $(\mathrm{SF})$ is measured from the ratio of the data and $\mathrm{MC}$ muonic $b$-jet TRFs.

\footnotetext{
${ }^{6}$ Muonic $b$-jets are used to measure the $b$-jet TRF for data, see Section 4.6.1.

${ }^{7}$ This is due to the presence of a high $\mathrm{p}_{T}$ track from the muon.
} 

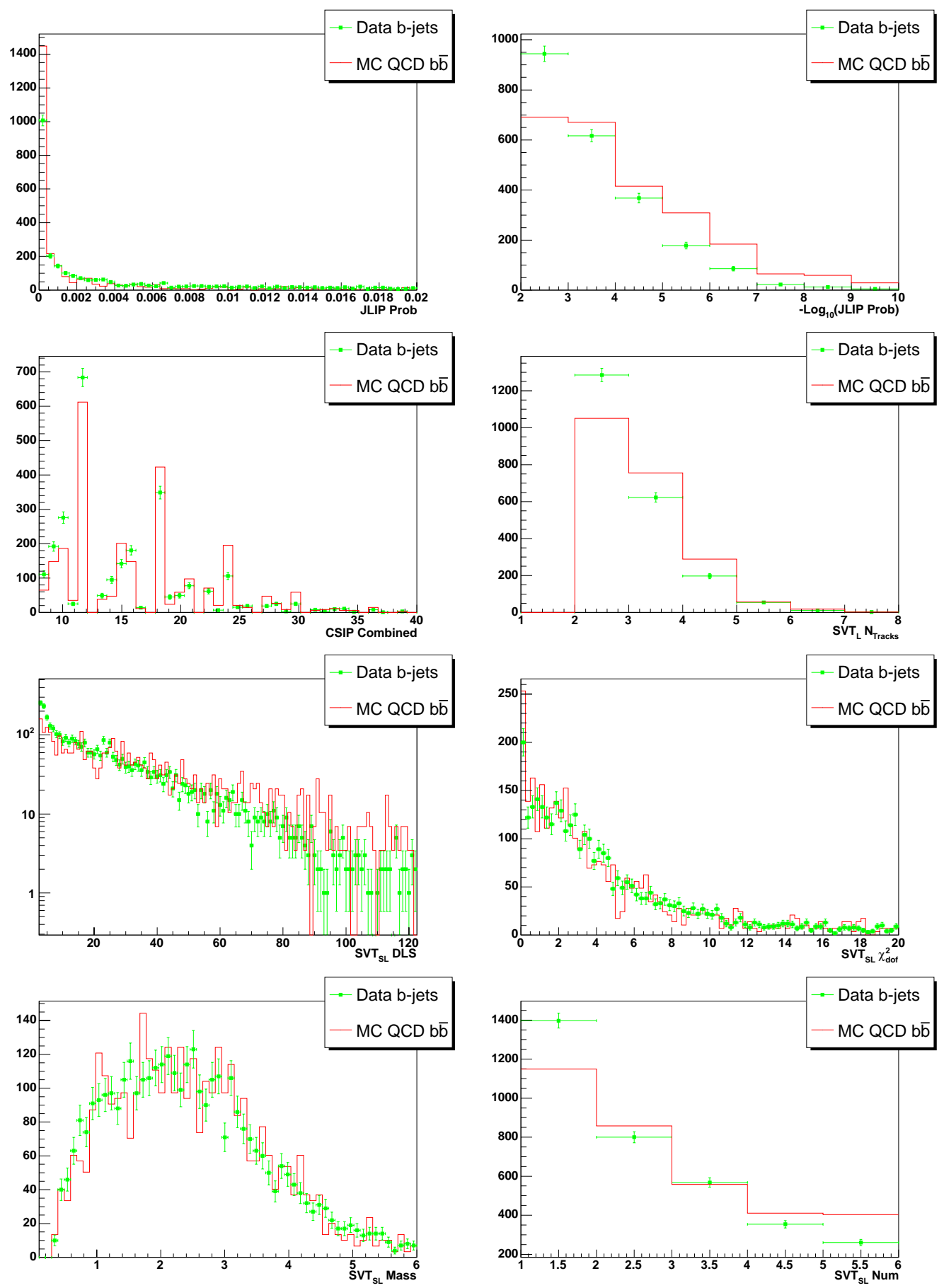

Figure 4.17: NN input variables in QCD $b \bar{b}$ (red line) $\mathrm{MC}$ and the $b$-enriched data sample (green squares) after the NN jet input cuts. The variables are JLIP Prob (top left), $-\log _{10}$ (JLIP Prob)(top right), CSIP Comb (2nd left), $\mathrm{SVT}_{L} N_{\text {Tracks }}$ (2nd right), $\mathrm{SVT}_{S L}$ DLS (3rd left), $\mathrm{SVT}_{S L}$ $\chi_{d o f}^{2}$ (3rd right), $\mathrm{SVT}_{S L}$ Mass (bottom left) and $\mathrm{SVT}_{S L}$ (bottom right). Comparisons should be made between the shape and not the absolute levels of the input variables, as their areas have been normalised to be equal. 

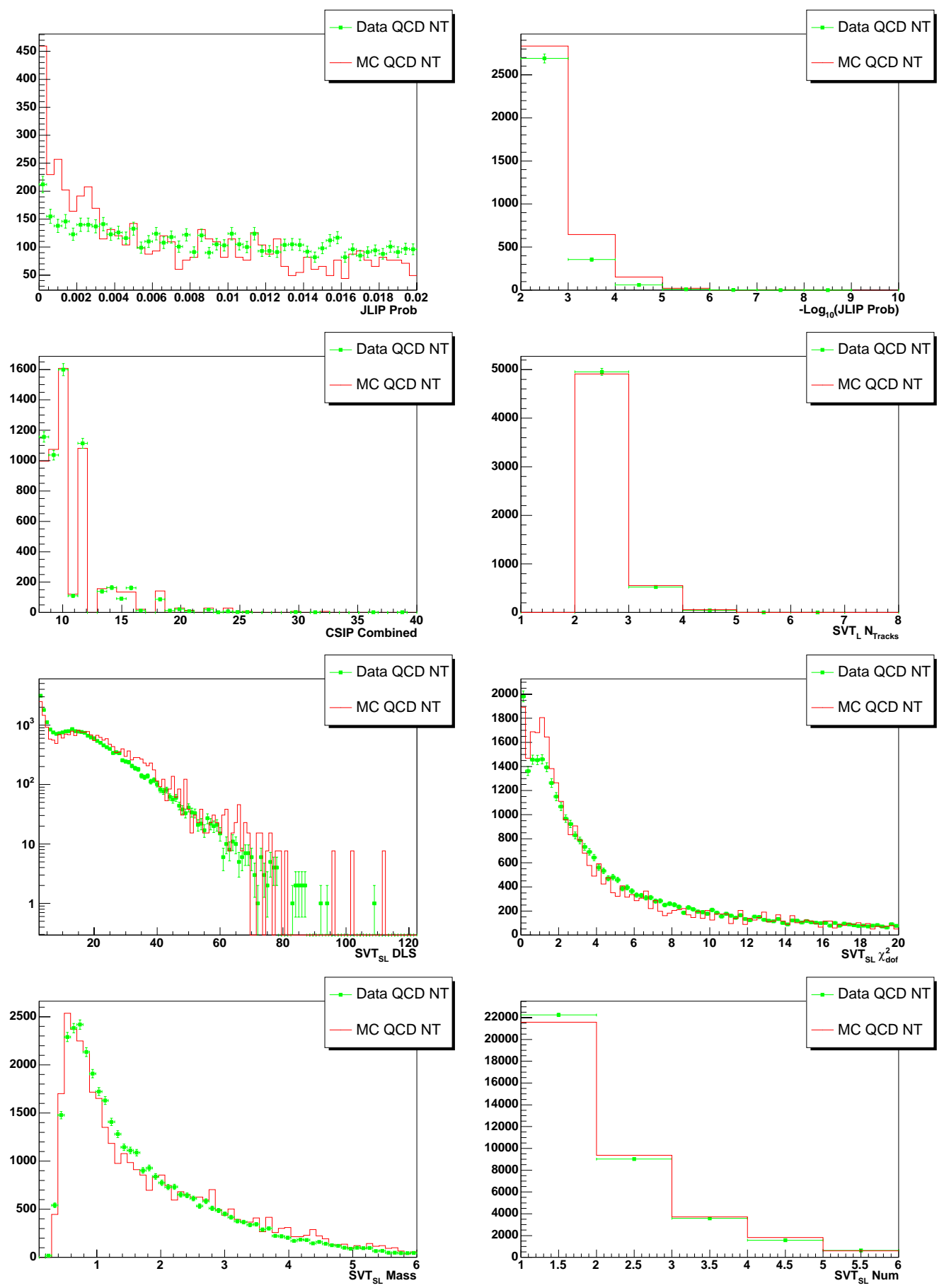

Figure 4.18: NN input NT variables in QCD MC (red line) and data (green squares) after the NN jet input cuts. The variables are JLIP Prob (top left), $-\log _{10}$ (JLIP Prob) (top right), CSIP Comb (2nd left), $\mathrm{SVT}_{L} N_{\text {Tracks }}$ (2nd right), $\mathrm{SVT}_{S L}$ DLS (3rd left), $\mathrm{SVT}_{S L} \chi_{\text {dof }}^{2}$ (3rd right), $\mathrm{SVT}_{S L}$ Mass (bottom left) and $\mathrm{SVT}_{S L}$ (bottom right). Comparisons should be made between the shape and not the absolute levels of the input variables, as their areas have been normalised to be equal. 

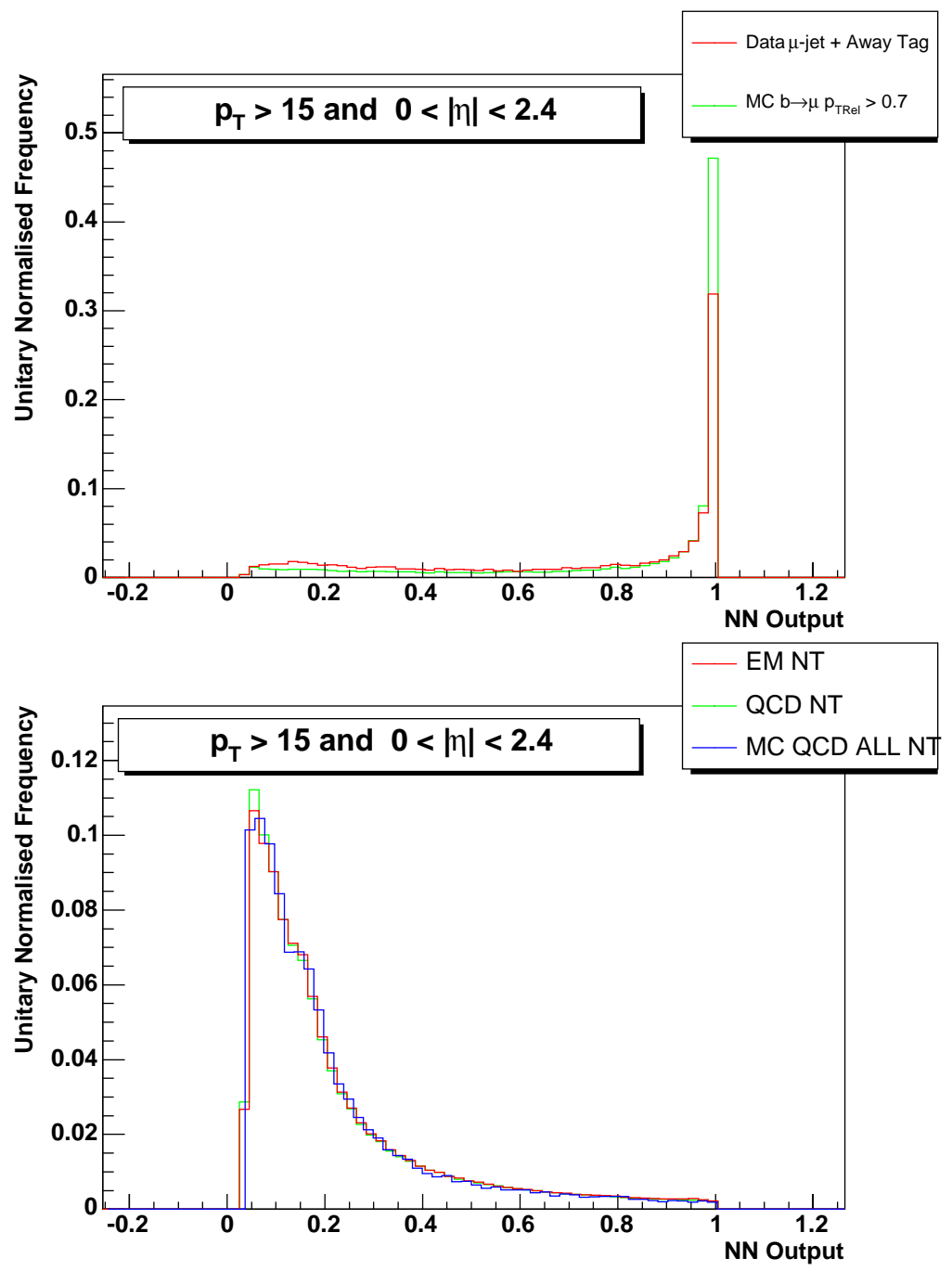

Figure 4.19: NN output data/MC comparison: Top - The b-enriched skim (red) and QCD $b \bar{b}$ MC (green). Bottom - The NT outputs from the QCD (green) and EM (red) data skims compared to the QCD MC sample (blue). Comparisons should be made between the shape and not the absolute levels of the NN outputs, as their areas have been normalised to be equal. 
4. Inclusive $b$ and $c$-jet TRFs are measured for MC.

5. Inclusive $b$ and $c$-jet TRFs for data are produced by multiplying the inclusive MC $b$ and $c$-jet TRFs by the $\mathrm{SF}^{8}$.

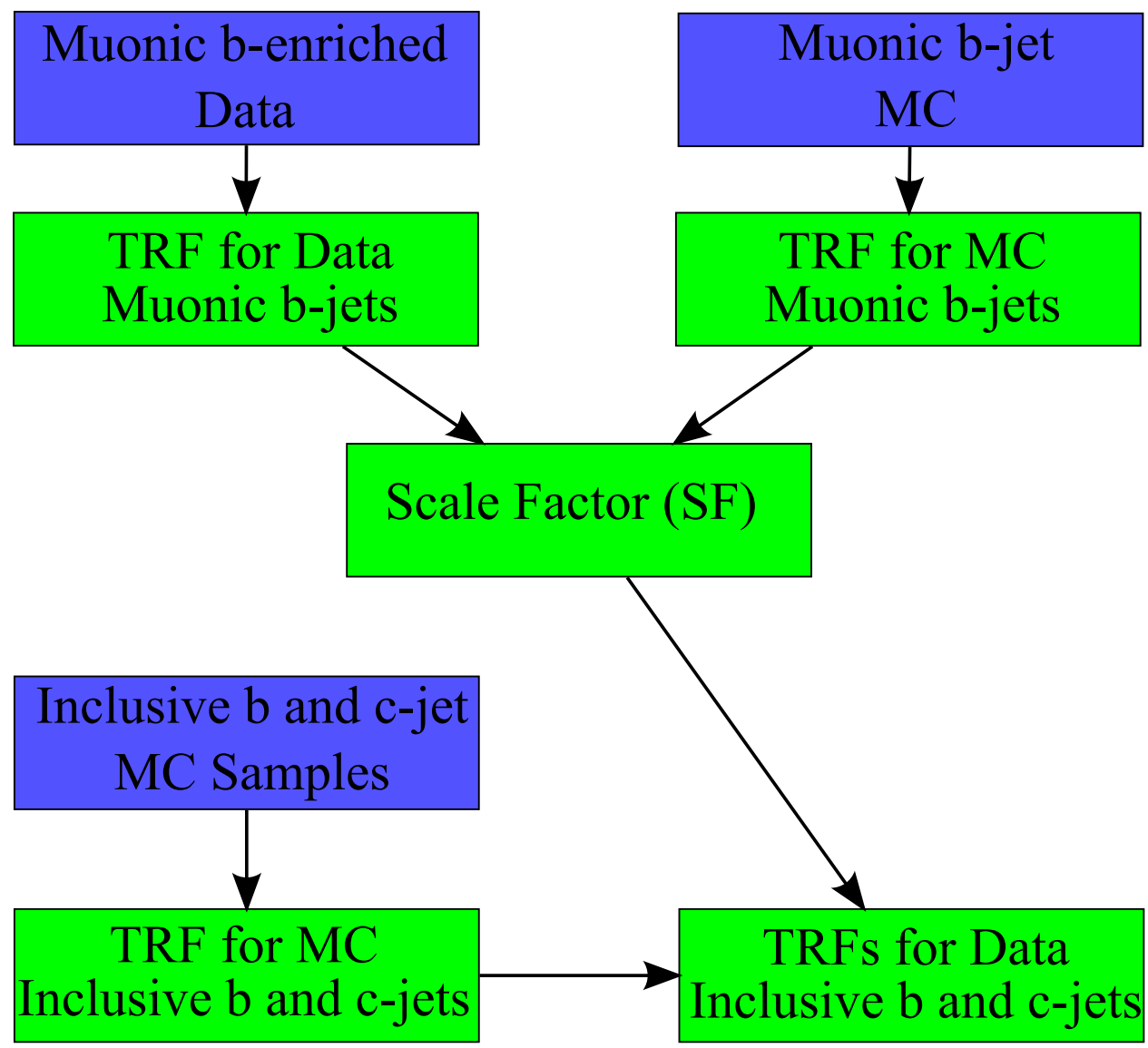

Figure 4.20: The process to measure the TRFs for inclusive $b$ and $c$-jets.

The measurement of the muonic $b$-jet data TRF is described in Sections 4.6.1 4.6.4, the data/MC scale factor in Section 4.6.5, the TRFs for inclusive $b$ and $c$-jets in Section 4.6.6 and the errors on the TRFs in Section 4.6.7.

\subsubsection{System8 Method}

The $b$-jet efficiency on data was measured using the System8 (S8) formalism [86] which is the official D $\varnothing$ method for measuring the $b$-efficiency on data. A system of 8 equations with 8 unknowns is constructed by equating the number of tags found by two different taggers on two different samples. The solutions to the equations include the $b$-tagging efficiencies of the two taggers.

\footnotetext{
${ }^{8}$ The $\mathrm{D} \varnothing$ certification assumes that the scale factor is the same for inclusive and muonic $b$-jets.
} 
In order that the equations are solvable the two taggers need to be uncorrelated and have different $b$-efficiencies, the two data samples also need to have different $b$-content.

The NN and SLT taggers are used as the two taggers. A SLT tag was defined as a muonic jet where the muon satisfied a $p_{T \text { Rel }}$ cut $>0.6 \mathrm{GeV}$. The two data samples used were the $b$-ID muonic jet sample and a $b$-enriched subset consisting of muonic jets with an away tag of JLIP Prob $<0.01$.

Correlation coefficients are introduced in the S8 formulation to account for the following effects:

$\alpha$ - Differences in the NN $u d s c$-tagging efficiency between the two samples.

$\beta$ - Differences in the NN $b$-tagging efficiency between the two samples.

$\kappa_{b}$ - Correlation between the NN tagger and the SLT tagger for $b$-jets.

$\kappa_{u d s c}$ - Correlation between the NN tagger and the SLT tagger for udsc-jets.

There is an additional correlation coefficient, $\tau_{\text {slt }}$, which accounts for the differences between the SLT tagging efficiency on $c$ and fake-jets. This correlation coefficient is not included in the $\mathrm{S} 8$ formulation and is evaluated separately.

The correlation coefficients are measured using MC. The errors on these measurements are used to estimate systematic errors. The S8 equations are defined as:

$$
\begin{aligned}
n & =n_{b}+n_{u d s c} \\
p & =p_{b}+p_{u d s c} \\
n^{S L T} & =\varepsilon_{b}^{S L T} n_{b}+\varepsilon_{u d s c}^{S L T} n_{u d s c} \\
p^{S L T} & =\varepsilon_{b}^{S L T} p_{b}+\varepsilon_{u d s c}^{S L T} p_{u d s c} \\
n^{N N} & =\varepsilon_{b}^{N N} n_{b}+\varepsilon_{u d s c}^{N N} n_{u d s c} \\
p^{N N} & =\beta \varepsilon_{b}^{N N} p_{b}+\alpha \varepsilon_{u d s c}^{N N} p_{u d s c} \\
n^{S L T, N N} & =\kappa_{b} \varepsilon_{b}^{S L T} \varepsilon_{b}^{N N} n_{b}+\kappa_{u d s c} \varepsilon_{u d s c}^{S L T} \varepsilon_{u d s c}^{N N} n_{u d s c} \\
p^{S L T, N N} & =\kappa_{b} \beta \varepsilon_{b}^{S L T} \varepsilon_{b}^{N N} p_{b}+\kappa_{u d s c} \alpha \varepsilon_{u d s c}^{S L T} \varepsilon_{u d s c}^{N N} p_{u d s c},
\end{aligned}
$$

where $n$ is the number of jets in the muonic jet sample, $p$ is the number in the $b$-enriched sample and $\varepsilon$ is the efficiency of the tagger. The superscripts refer to the NN and SLT taggers and the subscripts refer to the flavour of the jets, $b$ or $u d s c$. 


\subsubsection{Measurement of the Correlation Coefficients}

\subsubsection{1 $\alpha$}

The correlation coefficient $\alpha$, representing the difference between the NN tagging efficiency for $u d s c$-jets in the two samples, is not calculated from MC due to the difficulty in producing the necessary MC samples. $\alpha$ is instead set equal to 1 , and the error due to the uncertainty in the value is estimated by varying this value from 0.2 to 1.8 .

\subsubsection{2 $\beta$}

$\beta$ represents any differences between the NN $b$-efficiencies in the two samples. $\beta$ was measured using the muonic $b$-jet MC, by calculating the ratio of the tagging efficiency for $b$-jets with an away $\operatorname{tag}^{9}$ to that for all $b$-jets, and is shown in Fig. 4.21 (top).
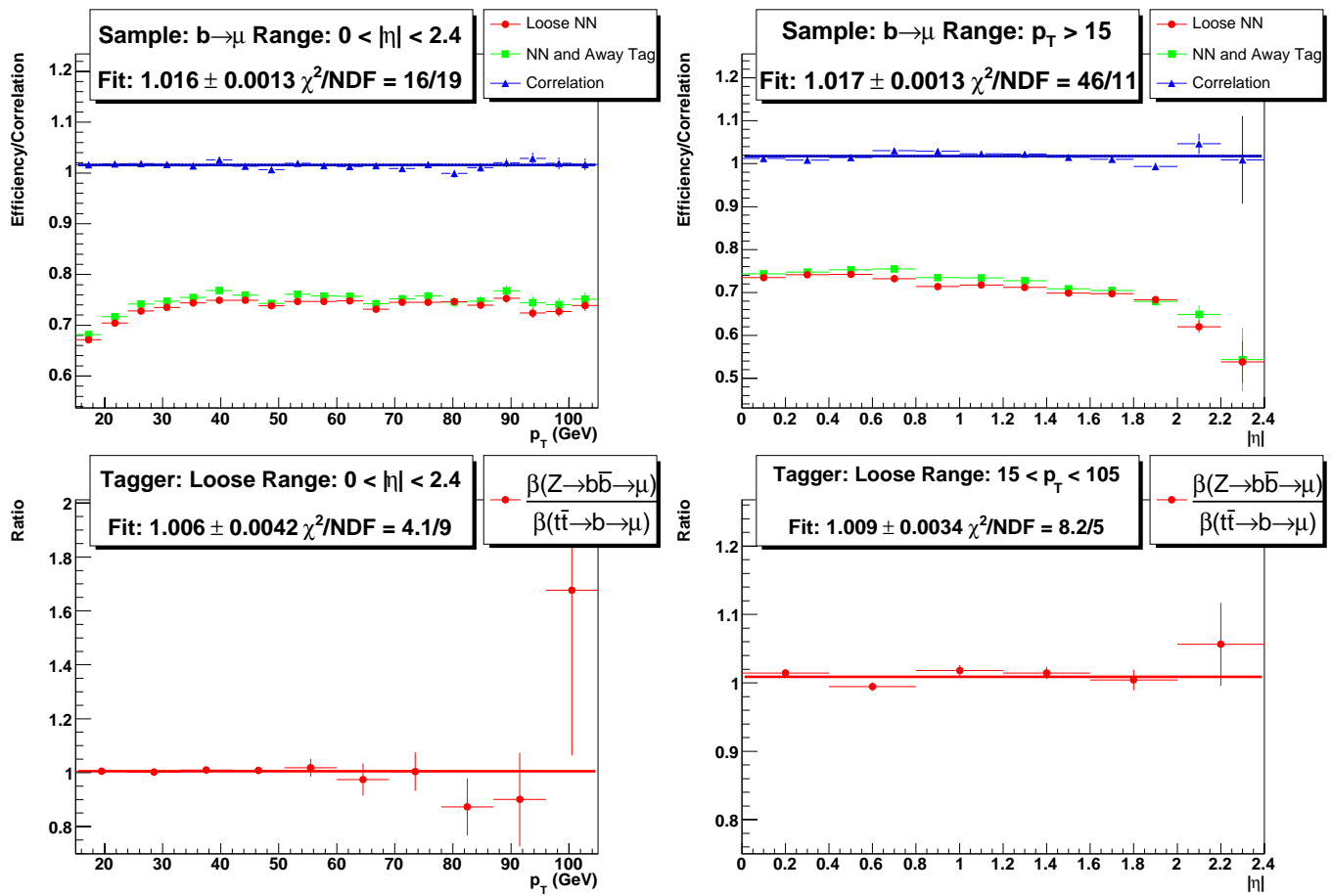

Figure 4.21: Top: The Loose NN tagging efficiency on muonic $b$-jets (green squares), the tagging efficiency on muonic $b$-jets with an away tag (red squares), and their ratio, $\beta$ (blue line) in the $b \rightarrow \mu \mathrm{MC}$ sample as a function of jet $p_{T}$ (left) and $\eta$ (right). The fit error on $\beta$ is represented by two black dotted lines although the error is to small for these to be seen. Bottom: The ratio between $\beta$ found in the $Z \rightarrow b \bar{b} \rightarrow \mu$ and $t \bar{t} \rightarrow b \rightarrow \mu$ samples in $\mathrm{p}_{T}$ (left) and $\eta$ (right) projections.

\footnotetext{
${ }^{9}$ An away tag is used to produce the $b$-enriched sub-sample (see Section 4.6.1)
} 
As well as the statistical uncertainty, another source of uncertainty exists due to the sample dependency of $\beta$. As shown in Fig. 4.21 (bottom) the ratio of the $\beta$ value calculated in the $Z \rightarrow b \bar{b}$ and the $t \bar{t} \rightarrow b$ samples is not unity. The uncertainty caused by the sample dependency is calculated by taking half the average deviation from unity in the $\eta$ and $\mathrm{p}_{T}$ projections. The sample dependency error is added to the statistical error in quadrature to give the total error on $\beta$.

\subsubsection{3 $\kappa$}

$\kappa_{b}$ and $\kappa_{u d s c}$ represent the correlations between the NN and SLT taggers, and are determined from the following relationship

$$
\kappa_{b, u d s c}=\frac{\varepsilon_{b, u d s c}^{N N} \times \varepsilon_{b, u d s c}^{S L T}}{\varepsilon_{b, u d s c}^{N N \& S L T}},
$$

where $\varepsilon$ is the efficiency, the subscripts refer to the jet flavour, the superscripts refer to the taggers and the ampersand to both tags being present.

$\kappa_{b}$ was measured in the muonic $b$-jet MC sample and is shown in Fig. 4.22 (top). A linear dependency exists in $\mathrm{p}_{T}$ and it is therefore modelled using a straight line. The error on the fit is determined from the full covariant error matrix and is therefore also a function of $\mathrm{p}_{T}$. As the fit value and error are a function of $\mathrm{p}_{T}$ the systematic error due to the uncertainty in the measurement of $\kappa_{b}$ also becomes a function of $\mathrm{p}_{T}$.

Another source of uncertainty exists due to the sample dependency of $\kappa_{b}$. As shown in Fig. 4.22 the ratio of the $\kappa_{b}$ value calculated in the $Z \rightarrow b \bar{b}$ and the $t \bar{t} \rightarrow b$ samples is not unity. An uncertainty is estimated for this effect by taking half the average deviation from unity in the $\eta$ and $\mathrm{p}_{T}$ projections. The $\eta$ projection is restricted to a limited range in $\mathrm{p}_{T}$ due to the $\mathrm{p}_{T}$ dependence of $\kappa_{b}$ and the difference in the $\mathrm{p}_{T}$ spectra in the two samples. The sample dependency error is added to the statistical error in quadrature to give the total error on $\kappa_{b}$.

$\kappa_{u d s c}$ was measured in a similar manner to $\kappa_{b}$, but using the $c \rightarrow \mu$ MC sample. The statistical error was again added in quadrature with a sample dependency error, calculated from the $Z \rightarrow c \bar{c}$ and the $t \bar{t} \rightarrow c$ samples, to give the total error on $\kappa_{u d s c}$.

\subsubsection{4 $\tau_{s l t}$}

The correlation coefficient $\tau_{s l t}$, representing the differences between the SLT tagging efficiency on $c$ and $u d s g$-jets, is not represented in the $\mathrm{S} 8$ equations. Instead the uncertainty caused by this assumption is evaluated by recalculating the $\mathrm{S} 8$ efficiency at different SLT operating points. The SLT $p_{T \text { Rel }}$ cut is varied from 0.4 to 0.8 . The 

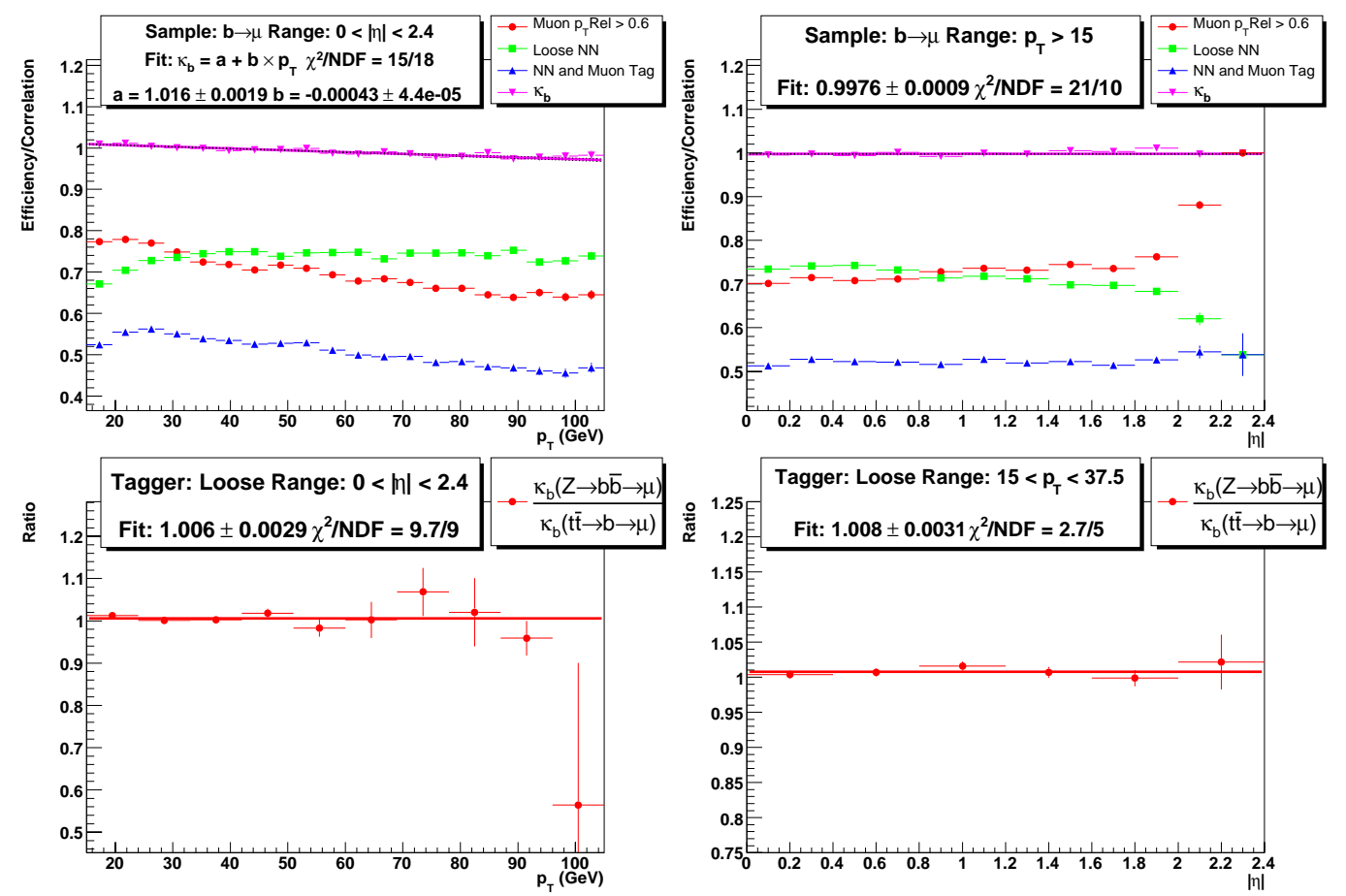

Figure 4.22: Top: The efficiency of the SLT tagger (red circles), the Loose NN tagger (green squares), the AND of the NN and SLT taggers (blue triangles), and $\kappa_{b}$ (upside down purple triangles), measured in the $b \rightarrow \mu \mathrm{MC}$ sample in the jet $p_{T}$ (left) and $\eta$ (right) projections. The black dotted line represents the error on the fit calculated from the full covariant fit error matrix. Bottom: The ratio of $\kappa_{b}$ measured in the $Z \rightarrow b \bar{b} \rightarrow \mu$ and $t \bar{t} \rightarrow b \rightarrow \mu$ MC samples in $\mathrm{p}_{T}$ (left) and $\eta$ (right).

values of $\kappa_{b}$ and $\kappa_{u d s c}$ are dependent on the value of the SLT $p_{T R e l}$ cut and therefore both these values are recalculated at each SLT operating point.

\subsubsection{Correlation Coefficient Values}

The values of the correlation coefficients are given in Table 4.11. The values and errors are consistent between the $\eta$ and $\mathrm{p}_{T}$ projections. As the $\kappa_{b}$ value and error are $\mathrm{p}_{T}$ dependent, the values are evaluated at the average jet $\mathrm{p}_{T}$ value for the sample.

\subsubsection{System8 Solution and Systematic Errors}

The S8 equations were solved using TMinuit [87], for all the jets in the $b$-ID sample and in several $\mathrm{p}_{T}$ and $\eta$ regions to create a profile over the $\mathrm{p}_{T}$ and $\eta$ phase space. The systematic uncertainties, due to the errors in the correlation coefficients, are determined by varying the coefficients by their aforementioned errors and resolving the equations. The relative differences in the efficiencies between the solutions provide the systematic errors. 


\begin{tabular}{|c|c|c|}
\hline Correlation Coefficient & $\mathrm{p}_{T}$ & $\eta$ \\
\hline$\alpha$ & $1.0 \pm 0.8$ & $1.0 \pm 0.8$ \\
$\beta$ & $1.016 \pm 0.004$ & $1.016 \pm 0.004$ \\
$\kappa_{b}$ & $1.000 \pm 0.003$ & $0.998 \pm 0.003$ \\
$\kappa_{u d s c}$ & $0.970 \pm 0.026$ & $0.975 \pm 0.026$ \\
$\tau_{\text {slt }}(\mathrm{GeV})$ & $0.6 \pm 0.2$ & $0.6 \pm 0.2$ \\
\hline
\end{tabular}

Table 4.11: The S8 correlation coefficient values for the Loose operating point measured in the $\mathrm{p}_{T}$ and $\eta$ projections. $\kappa_{u d s c}$ is evaluated at $\mathrm{p}_{T}=39 \mathrm{GeV}$ in the $\mathrm{p}_{T}$ projection (the average $\mathrm{p}_{T}$ of the sample). The values measured in the $\mathrm{p}_{T}$ and $\eta$ projections are consistent within errors.

The S8 efficiencies along with the systematic errors calculated for the $b$-ID sample are shown in Table 4.12. The efficiency ranges from $50 \%$ for the tightest operating point to $70 \%$ for the loosest operating point. The relative systematic uncertainties vary from $1.5-2.1 \%$.

\begin{tabular}{|c|c|c|c|c|c|c|}
\hline & L4 & L3 & L2 & Loose & Medium & Tight \\
\hline Efficiency & $71.4 \%$ & $68.8 \%$ & $65.3 \%$ & $59.0 \%$ & $54.0 \%$ & $48.6 \%$ \\
\hline$\alpha$ & $1.70 \%$ & $1.14 \%$ & $0.81 \%$ & $0.73 \%$ & $0.85 \%$ & $0.69 \%$ \\
$\beta$ & $0.31 \%$ & $0.39 \%$ & $0.41 \%$ & $0.48 \%$ & $0.45 \%$ & $0.65 \%$ \\
$\kappa_{b}$ & $0.55 \%$ & $0.75 \%$ & $0.67 \%$ & $1.13 \%$ & $1.19 \%$ & $1.21 \%$ \\
$\kappa_{\text {udsc }}$ & $0.80 \%$ & $0.71 \%$ & $0.75 \%$ & $0.29 \%$ & $0.15 \%$ & $0.16 \%$ \\
$\tau_{\text {slt }}$ & $0.70 \%$ & $0.78 \%$ & $0.69 \%$ & $0.63 \%$ & $0.67 \%$ & $0.49 \%$ \\
\hline Total & $2.11 \%$ & $1.76 \%$ & $1.52 \%$ & $1.59 \%$ & $1.67 \%$ & $1.61 \%$ \\
\hline
\end{tabular}

Table 4.12: The $b$-efficiencies and their relative systematic uncertainties measured on the $b$-ID skim. The total systematic uncertainty was determined by adding the individual uncertainties in quadrature.

\subsubsection{Efficiency and Scale Factor Parameterisation}

The efficiency (and scale factor) in $\mathrm{p}_{T}$ is parameterised using

$$
\varepsilon\left(p_{T}\right)=\frac{c}{1+a e^{-b p_{T}}},
$$

where $\varepsilon$ is the efficiency and $a, b$, and $c$ are constants to be determined. The efficiency is parameterised in $\eta$ using 


$$
\varepsilon(\eta)=d+e \eta+f \eta^{2}+g \eta^{3}+h \eta^{4},
$$

where $\varepsilon$ is the efficiency and $d, e, f, g$ and $h$ are constants to be determined. To create a $2 \mathrm{D}$ parameterisation it is assumed that the efficiency can be factorised into $\eta$ and $\mathrm{p}_{T}$ components. A $2 \mathrm{D}$ parameterisation is created from the product of the two projections scaled by the overall efficiency, $\varepsilon^{A l l}$, of the sample

$$
\varepsilon\left(p_{T}, \eta\right)=\frac{1}{\varepsilon^{A l l}}\left(\frac{c}{1+a e^{-b p_{T}}}\right) \times\left(d+e \eta+f \eta^{2}+g \eta^{3}+h \eta^{4}\right) .
$$

These functions were selected after testing several different functions in both the $\mathrm{p}_{T}$ and $\eta$ projections, and selecting the functions which gave the smallest fit error.

\subsubsection{Data/MC Scale Factor}

The data efficiency for the $b$-ID skim calculated from the S8 equations is shown in Fig. 4.23, along with the MC $b \rightarrow \mu$ sample efficiency and the data/MC SF produced by dividing the two. The SF measures the difference in the tagging rate caused by the various tagging differences in data and MC. The profile of the SF allows tagging rates to be corrected over the full $\mathrm{p}_{T}$ and $\eta$ phase space.

It was assumed that the SF could be applied to any MC tagging efficiency to correct to the data tagging efficiency. This assumption allows the SF, which is measured only on muonic jets, to be applied to both muonic and inclusive jets. The validity of this assumption has not been tested and it is assumed that any effect will be small in relation to the other errors. Further studies into this systematic error were planned in the next round of certification.

The $\mathrm{S} 8$ errors evaluated on a bin-by-bin basis in the $\mathrm{p}_{T}$ and $\eta$ projections are shown in Fig. 4.24 for the Loose operating point. Evaluating the S8 errors in each bin produces a larger systematic error than calculating the $\mathrm{S} 8$ errors for the entire sample. The S8 errors have very little $\eta$ dependence, apart from at high $\eta$ where the $\tau_{\text {slt }}$ uncertainty is substantially larger. The $\mathrm{S} 8$ error calculated for each bin was combined quadratically with the Minuit fit error on the $b$-efficiency to provide the total uncertainty on the $b$-efficiency for each bin.

\subsubsection{Tag Rate Functions}

The inclusive $b$-jet TRF $\left(\mathrm{TRF}_{b}\right)$ for data is calculated by multiplying the inclusive $b$-jet MC TRF (produced from the inclusive $b$-jet MC sample) by the SF. The $\mathrm{TRF}_{b} \mathrm{~S}$ for data and MC are shown in Fig. 4.25. 

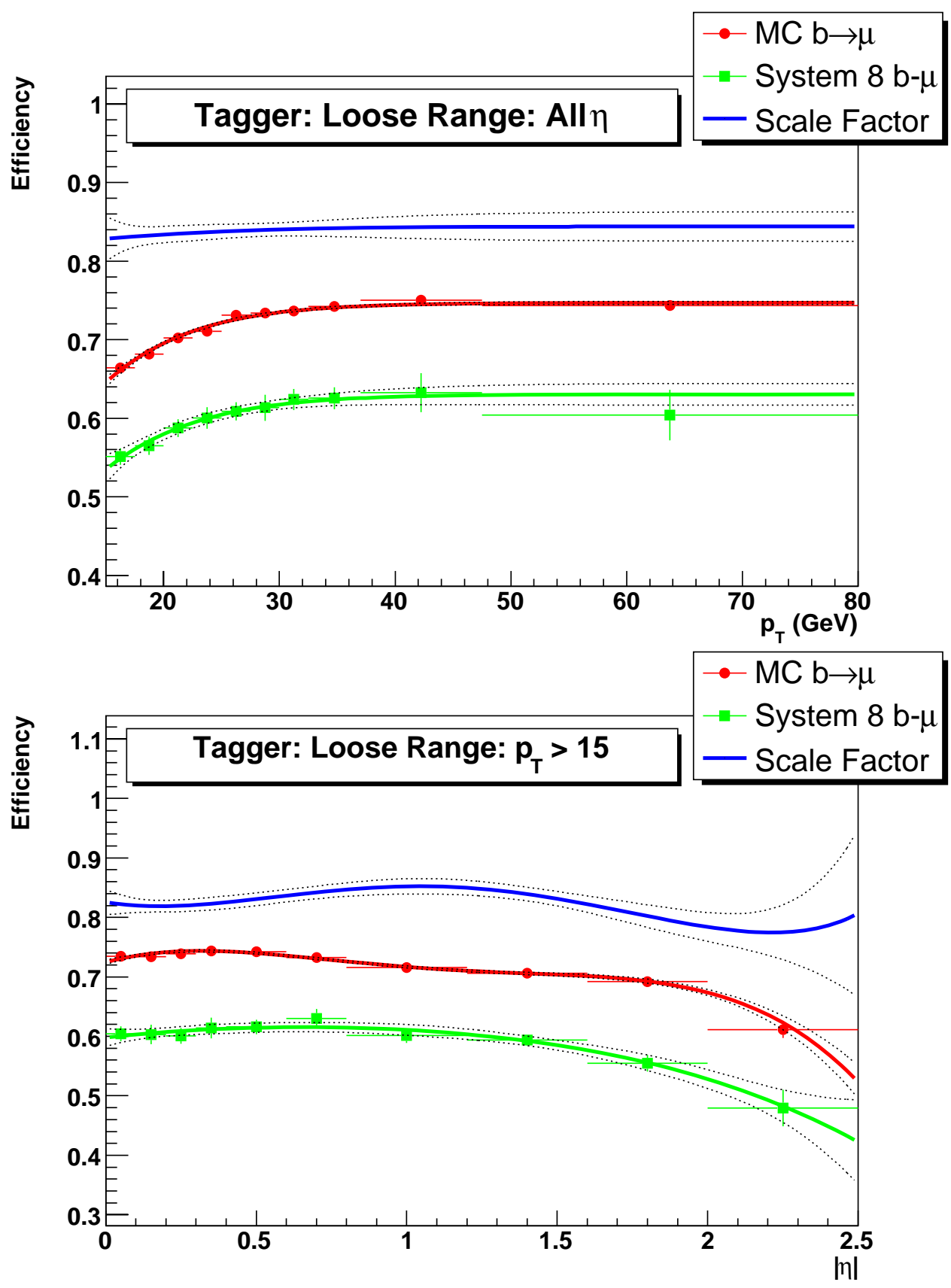

Figure 4.23: The Loose NN tagger scale factor (blue line) and the data (green squares) and MC (red circles) $b$-jet efficiencies as a function of $\mathrm{p}_{T}$ (top) and $\eta$ (bottom). The functions used for the parameterisation are outlined in the text and the black dotted curves represent the $\pm 1 \sigma$ statistical error. 

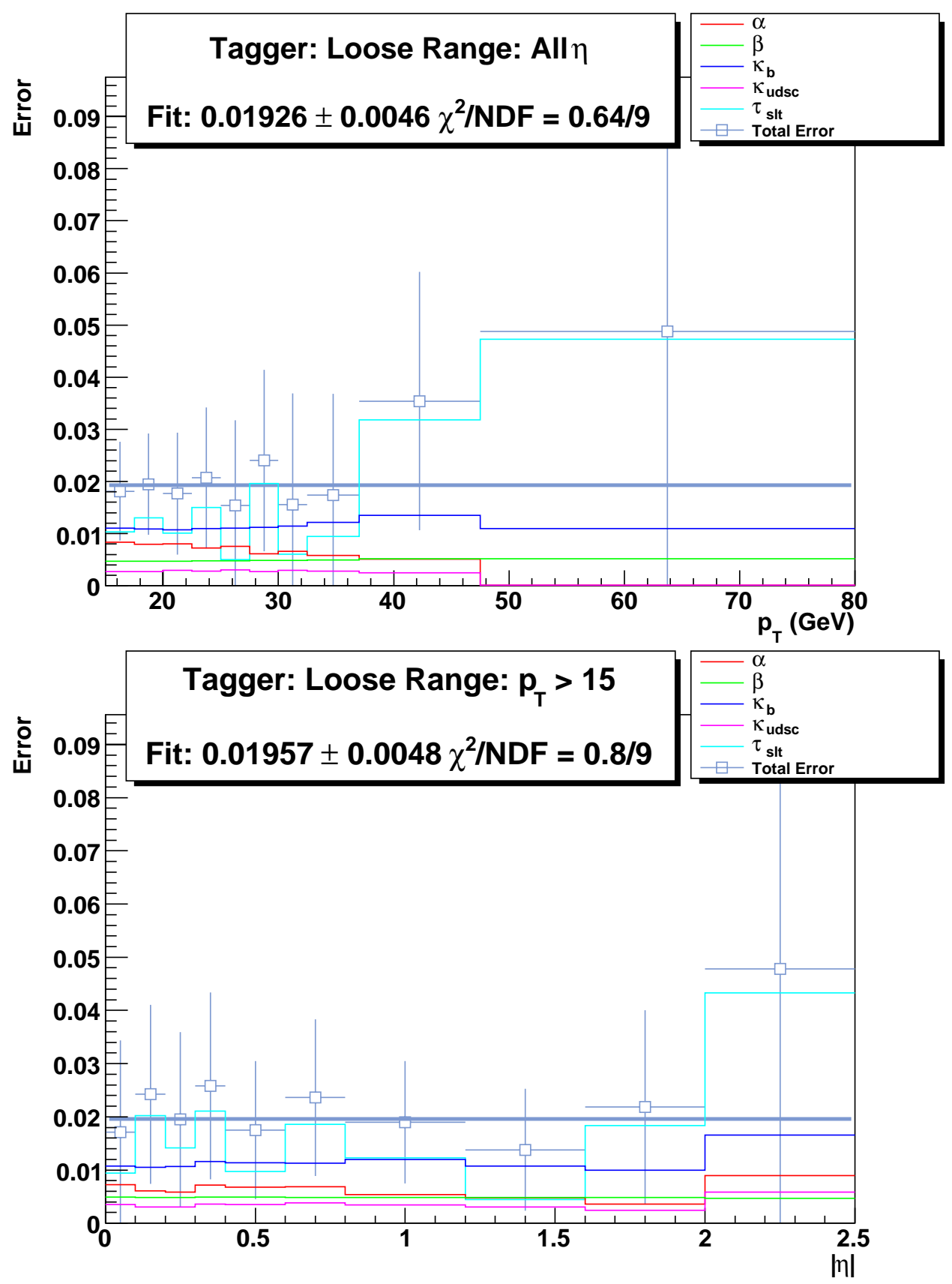

Figure 4.24: The $\alpha$ (red), $\beta$ (green), $\kappa_{b}$ (blue), $\kappa_{u d s c}$ (purple), $P_{T R e l}$ (cyan) and total combined (empty grey squares) S8 relative systematic errors calculated for each bin in $\mathrm{p}_{T}$ (left) and $\eta$ (right) for the Loose operating point. The grey line is a constant fit to the total combined error. 
A similar procedure is used to determine the inclusive $c$-jet $\mathrm{TRF}\left(\mathrm{TRF}_{c}\right)$ for data, the inclusive $c$-jet MC TRF (produced from the inclusive $c$-jet MC sample) is multiplied by the SF to give the data $\mathrm{TRF}_{c} \mathrm{~s}$ as also shown in Fig. 4.25 .
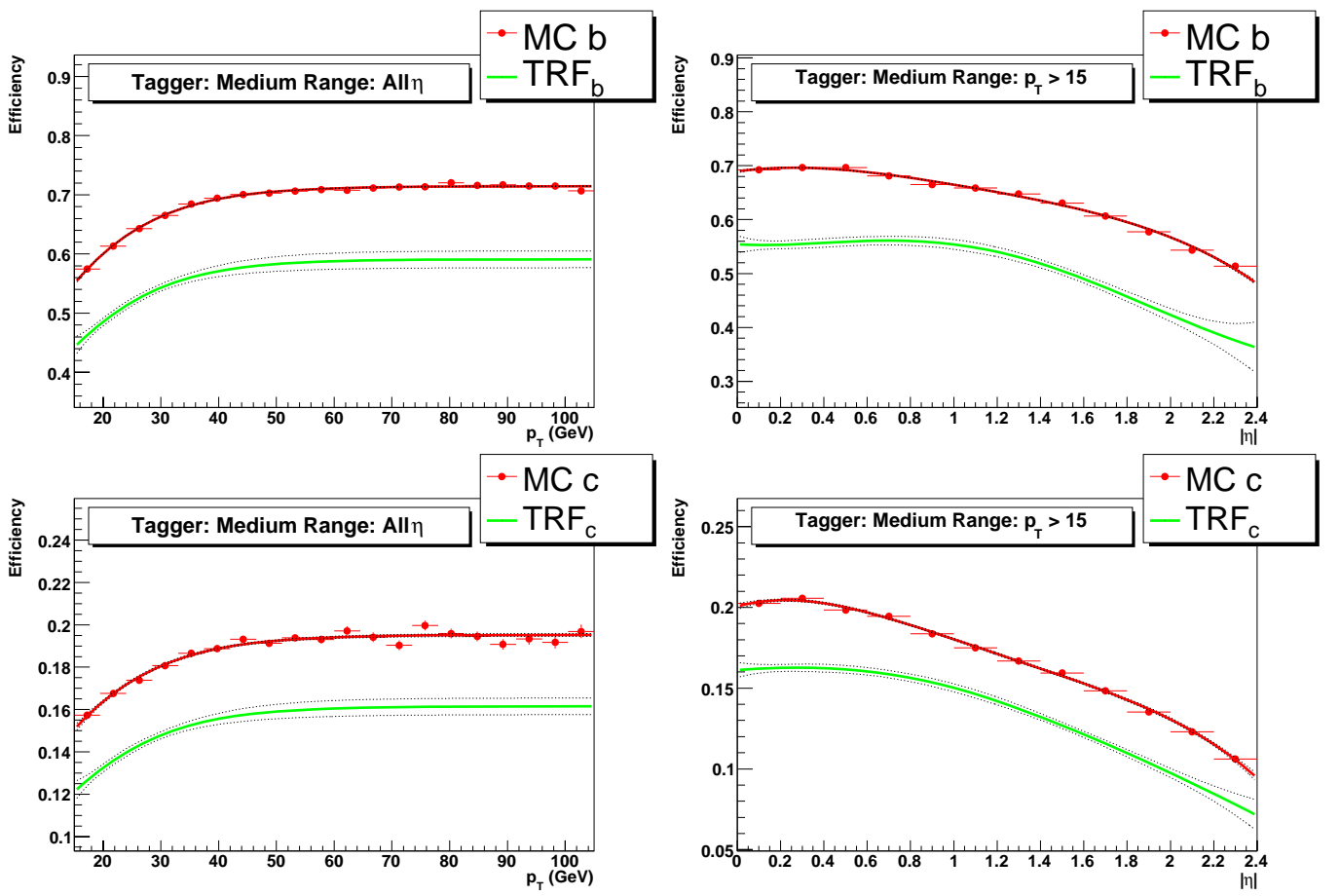

Figure 4.25: NN tagger inclusive $b$-jet efficiency (top) and $c$-jet efficiency (bottom) as a function of $\mathrm{p}_{T}$ (left) and $\eta$ (right) in both data (green line) and MC (red circles). The data TRF is calculated by multiplying the MC TRF by the data/MC SF. The dotted black lines represent the error on the fit which is almost entirely inherited from the error on the scale factor. The functions used for the parameterisation are outlined in the text.

\subsubsection{Systematic Uncertainties}

It was assumed that there were two main sources of error in the TRFs:

Parameterisation Error - The parameterisation of the efficiency into $\eta$ and $\mathrm{p}_{T}$ has a systematic uncertainty attributed to it, due to possible correlations between $\mathrm{p}_{T}$ and $\eta$ and the non-perfect parameterisations.

Sample Dependency - Differences exist between the tagging efficiencies in the different samples and therefore a systematic uncertainty exists due to these differences. 


\subsubsection{Closure Tests}

The systematic errors from the parameterisation and MC sample dependency are both quantified in one measurement. The uncertainty is measured using the fractional difference between the number of actual $\left(T_{O b s}\right)$ and predicted $\left(T_{\text {Pred }}\right)$ tags in various bins in $\mathrm{p}_{T}$ and $\eta$ measured on the various MC samples:

$$
\text { Fractional Diff }=\frac{T_{\text {Obs }}-T_{\text {Pred }}}{T_{\text {Obs }}} .
$$

Percentage differences are calculated in each $\mathrm{p}_{T}$ bin in the CC, ICR and EC calorimeter regions, for each TRF's component MC samples. For each MC sample a distribution is constructed from the fractional differences weighted by $T_{O b s}$. The root mean squared (RMS) of the resulting distribution is then used as an estimate of the uncertainty on the TRF for that particular MC sample. The fractional differences are shown in Fig. 4.26 for two representative MC samples from the inclusive $b$-jet TRF for the Loose operating point. Similar plots have been produced for the $c$-jet and muonic $b$-jet TRFs.

The errors derived from the RMS of the closure tests are presented in Tables $4.13,4.14$ and 4.15 for the $b$, muonic $b$ and $c$-jet TRFs respectively. The final systematic uncertainty for each TRF was taken from the MC sample with the largest uncertainty. The total uncertainty estimated from the closure test was larger than the combined parameterisation and sample dependency uncertainty when they were measured individually [74].

\begin{tabular}{|c|c|c|c|c|c|c|}
\hline Error & L4 & L3 & L2 & Loose & Medium & Tight \\
\hline$b$ & $0.94 \%$ & $1.03 \%$ & $1.16 \%$ & $1.36 \%$ & $1.53 \%$ & $1.75 \%$ \\
\hline QCD $b \bar{b}$ & $0.95 \%$ & $0.98 \%$ & $1.13 \%$ & $1.42 \%$ & $1.58 \%$ & $1.78 \%$ \\
$t \bar{t} \rightarrow b$ & $1.05 \%$ & $1.12 \%$ & $1.25 \%$ & $1.4 \%$ & $1.55 \%$ & $1.78 \%$ \\
$Z \rightarrow b \bar{b}$ & $1.04 \%$ & $1.12 \%$ & $1.21 \%$ & $1.41 \%$ & $1.49 \%$ & $1.66 \%$ \\
\hline
\end{tabular}

Table 4.13: The $b$-jet TRF error for the $b$-jet sample and each of the constituent MC samples. The sample with the largest error is used as the error on the TRF.

\subsubsection{Total Systematic Errors}

The final systematic errors for $\mathrm{TRF}_{b}, \mathrm{TRF}_{c}$ and the $\mathrm{SF}$ are calculated as detailed below, and are shown in Table 4.16.

SF - The RMS closure test error for the $b \rightarrow \mu$ TRF. 

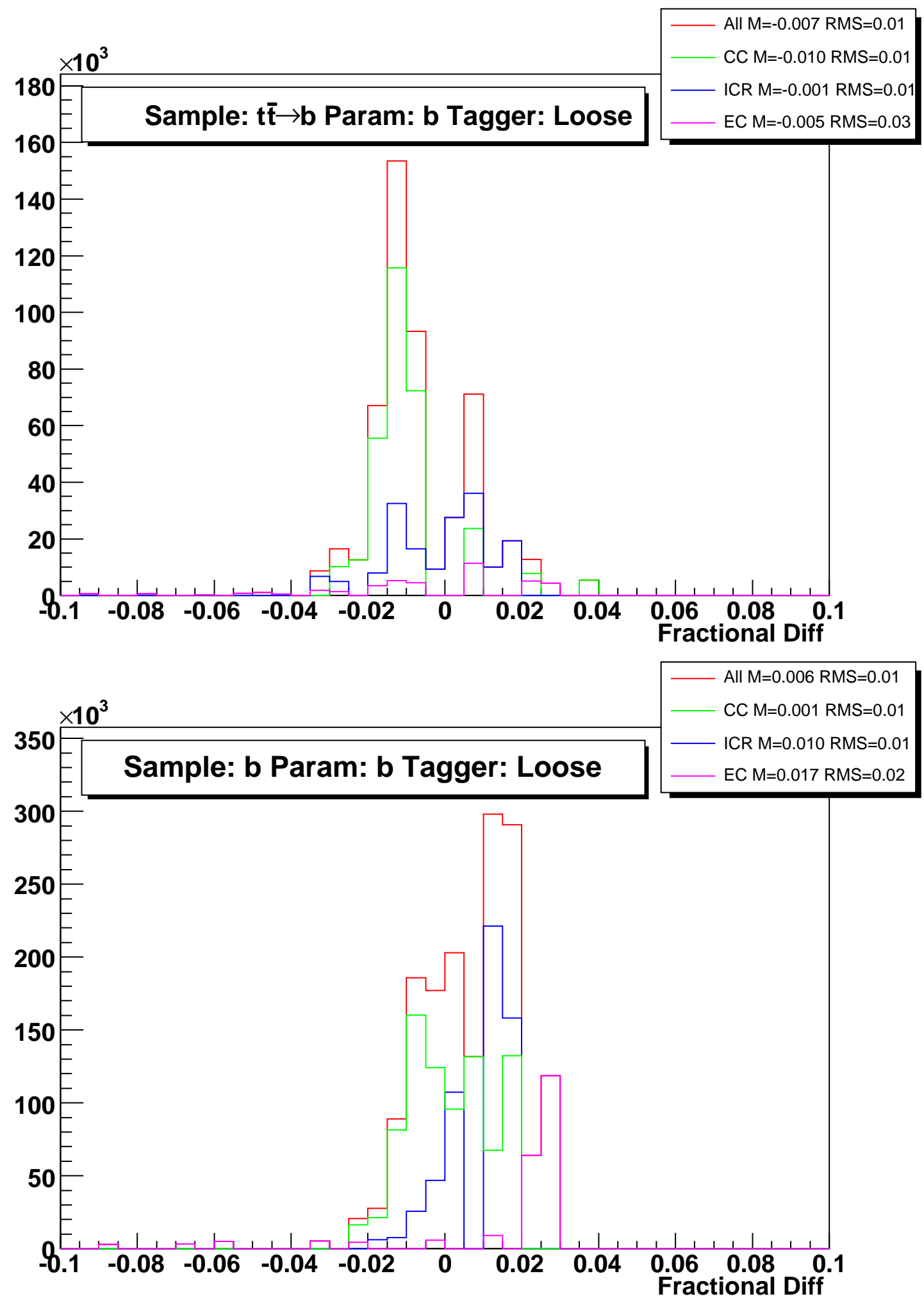

Figure 4.26: The fractional differences between the actual and predicted tags calculated in $\mathrm{p}_{T}$ bins in the CC (green), ICR (blue), EC (purple) and all (red) for the $t \bar{t} \rightarrow b$ (top) and the $b$ sample (bottom). The Mean (M) and root mean square (RMS) of the distributions are in the legend. The fractional errors are weighted by the number of actual tags in each bin and the RMS is used to estimate the error on the $b$-jet TRF. 


\begin{tabular}{|c|c|c|c|c|c|c|}
\hline Error & L4 & L3 & L2 & Loose & Medium & Tight \\
\hline$b \rightarrow \mu$ & $1.00 \%$ & $1.10 \%$ & $1.13 \%$ & $1.34 \%$ & $1.57 \%$ & $1.74 \%$ \\
\hline QCD $c \bar{c} \rightarrow \mu$ & $1.41 \%$ & $1.51 \%$ & $1.62 \%$ & $2.03 \%$ & $2.30 \%$ & $2.44 \%$ \\
$t \bar{t} \rightarrow b \rightarrow \mu$ & $1.63 \%$ & $1.72 \%$ & $1.86 \%$ & $2.22 \%$ & $2.56 \%$ & $2.92 \%$ \\
$Z \rightarrow b \bar{b} \rightarrow \mu$ & $1.14 \%$ & $1.13 \%$ & $1.10 \%$ & $1.32 \%$ & $1.48 \%$ & $1.66 \%$ \\
\hline
\end{tabular}

Table 4.14: The muonic $b$-jet TRF error for the muonic $b$-jet sample and each of the constituent MC samples. The sample with the largest error is used as the error on the TRF.

\begin{tabular}{|c|c|c|c|c|c|c|}
\hline Error & L4 & L3 & L2 & Loose & Medium & Tight \\
\hline$c$ & $1.66 \%$ & $1.7 \%$ & $1.86 \%$ & $2.25 \%$ & $2.53 \%$ & $2.79 \%$ \\
\hline QCD $c \bar{c}$ & $2.03 \%$ & $2.01 \%$ & $2.14 \%$ & $2.59 \%$ & $2.73 \%$ & $2.86 \%$ \\
$t \bar{t} \rightarrow c \rightarrow \mu$ & $4.08 \%$ & $4.05 \%$ & $4.04 \%$ & $3.59 \%$ & $4.17 \%$ & $3.52 \%$ \\
$Z \rightarrow c \bar{c}$ & $1.89 \%$ & $1.85 \%$ & $1.86 \%$ & $2.25 \%$ & $2.80 \%$ & $2.89 \%$ \\
\hline
\end{tabular}

Table 4.15: The $c$-jet TRF error for the $c$-jet sample and each of the constituent MC samples. The sample with the largest error is used as the error on the TRF.

$\mathbf{T R F}_{b}$ - The SF systematic error added in quadrature with the RMS error for the $b$-jet TRF.

$\mathbf{T R F}_{c}-$ The SF systematic error added in quadrature with the RMS error for the $c$-jet TRF.

\begin{tabular}{|c|c|c|c|c|c|c|}
\hline Error & L4 & L3 & L2 & Loose & Medium & Tight \\
\hline $\mathrm{MC} b \rightarrow \mu$ & $1.63 \%$ & $1.72 \%$ & $1.86 \%$ & $2.22 \%$ & $2.56 \%$ & $2.92 \%$ \\
$\mathrm{MC} b$ & $1.05 \%$ & $1.12 \%$ & $1.25 \%$ & $1.42 \%$ & $1.58 \%$ & $1.78 \%$ \\
$\mathrm{MC} c$ & $4.08 \%$ & $4.05 \%$ & $4.04 \%$ & $3.59 \%$ & $4.17 \%$ & $3.52 \%$ \\
\hline $\mathrm{SF}$ & $1.63 \%$ & $1.72 \%$ & $1.86 \%$ & $2.22 \%$ & $2.56 \%$ & $2.92 \%$ \\
$\mathrm{TRF}_{b}$ & $1.94 \%$ & $2.05 \%$ & $2.24 \%$ & $2.64 \%$ & $3.01 \%$ & $3.41 \%$ \\
$\mathrm{TRF}_{c}$ & $4.39 \%$ & $4.40 \%$ & $4.45 \%$ & $4.22 \%$ & $4.89 \%$ & $4.57 \%$ \\
\hline
\end{tabular}

Table 4.16: Total systematic errors on the MC sample parameterisations, the SF and the TRFs.

\subsubsection{Statistical Error}

The overall statistical error, $\sigma_{\text {stat }}$, is calculated by evaluating 


$$
\sigma_{\text {stat }}=\frac{f^{ \pm 1 \sigma}\left(p_{T}\right) \times f^{ \pm 1 \sigma}(\eta)}{f_{\text {All }}^{ \pm 1 \sigma}}-\frac{f\left(p_{T}\right) \times f(\eta)}{f_{\text {All }}},
$$

where $f$ is the parameterised fit in $\mathrm{p}_{T}$ and $\eta, f_{A l l}$ is the overall value of the parameterised value when calculated for the entire sample and $\pm 1 \sigma$ refers to the \pm 1 sigma statistical fits. The fluctuation which produces the largest deviation is taken as the statistical error.

\subsubsection{Total Error}

The total errors, given by the statistical and systematic errors combined in quadrature, for the SF, $\mathrm{TRF}_{b}$ and $\mathrm{TRF}_{c}$ are shown in Fig. 4.27 as a function of $\mathrm{p}_{T}$ and $\eta$. Statistical errors dominate at low $\mathrm{p}_{T}$ and high $\eta$ values, where the curves deviate from the constant systematic error values (outlined in Table 4.16).
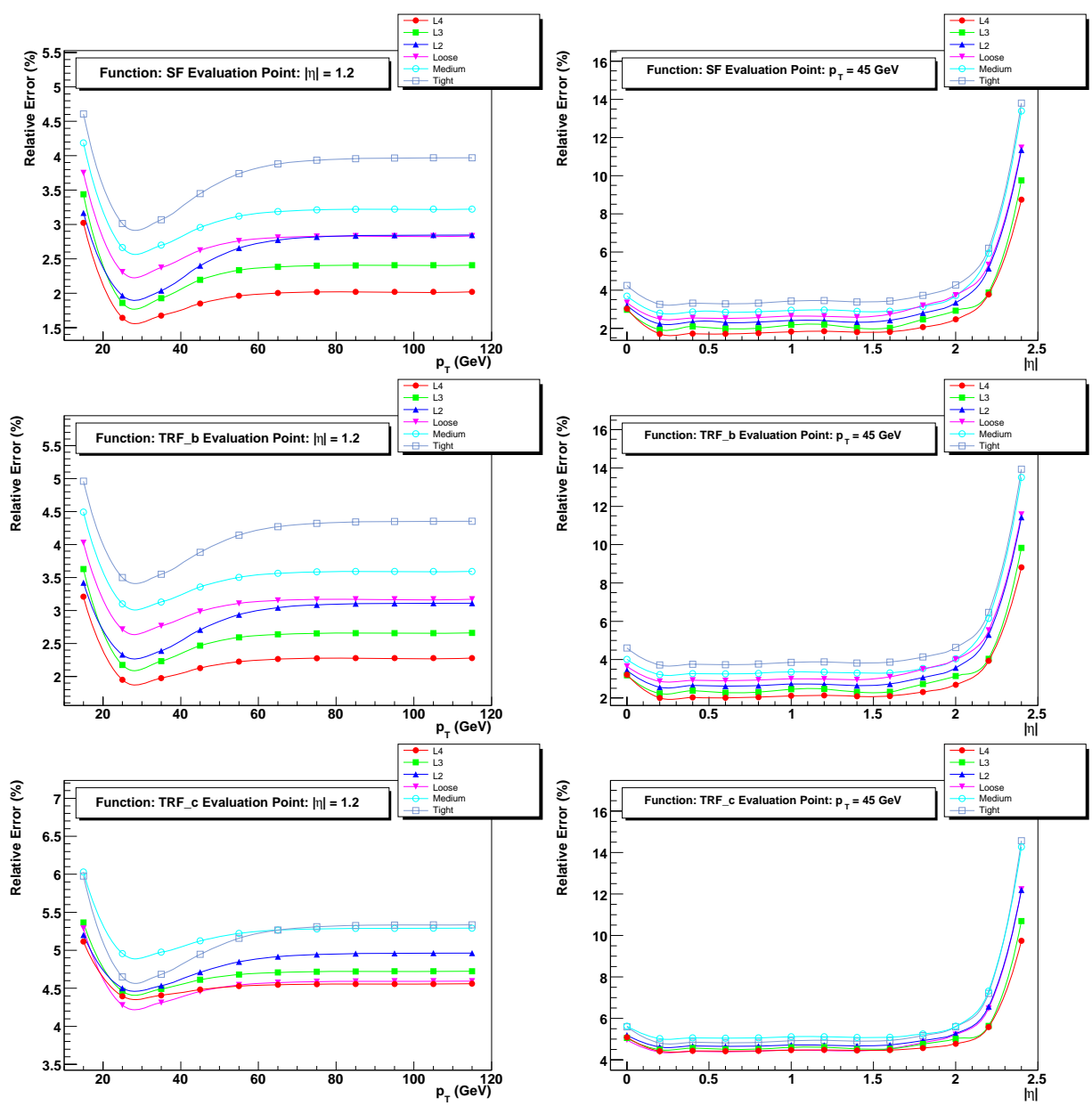

Figure 4.27: The total relative error (combined systematic and statistical) for the Scale Factor (SF) (top), $\mathrm{TRF}_{b}$ (middle) and $\mathrm{TRF}_{c}$ (bottom) for the L4 (red circles), L3 (green squares), L2 (blue triangles), Loose (purple triangles), Medium (cyan empty circles) and Tight (grey empty squares)

$\mathrm{NN}$ operating points in terms of $\mathrm{p}_{T}$ (left) when $\eta=1.2$ and $\eta$ (right) when $p_{T}=45 \mathrm{GeV}$. 


\subsection{Fake Rate}

The $\mathrm{D} \varnothing$ fake rate measurement is different to that used for the $b$ and $c$-jet efficiency measurements. The fake rate is measured, as illustrated in Fig. 4.28, by making use of the negative tags in data, which provide an approximate description of the fake rate (see Section 4.1.1.2). The negative tag rate (NTR) is corrected to the fake rate by NT scale factors, which correct for the presence of heavy flavour in the NTs, and for the asymmetry between the NTs and PTs caused by long lived 'light' particles.

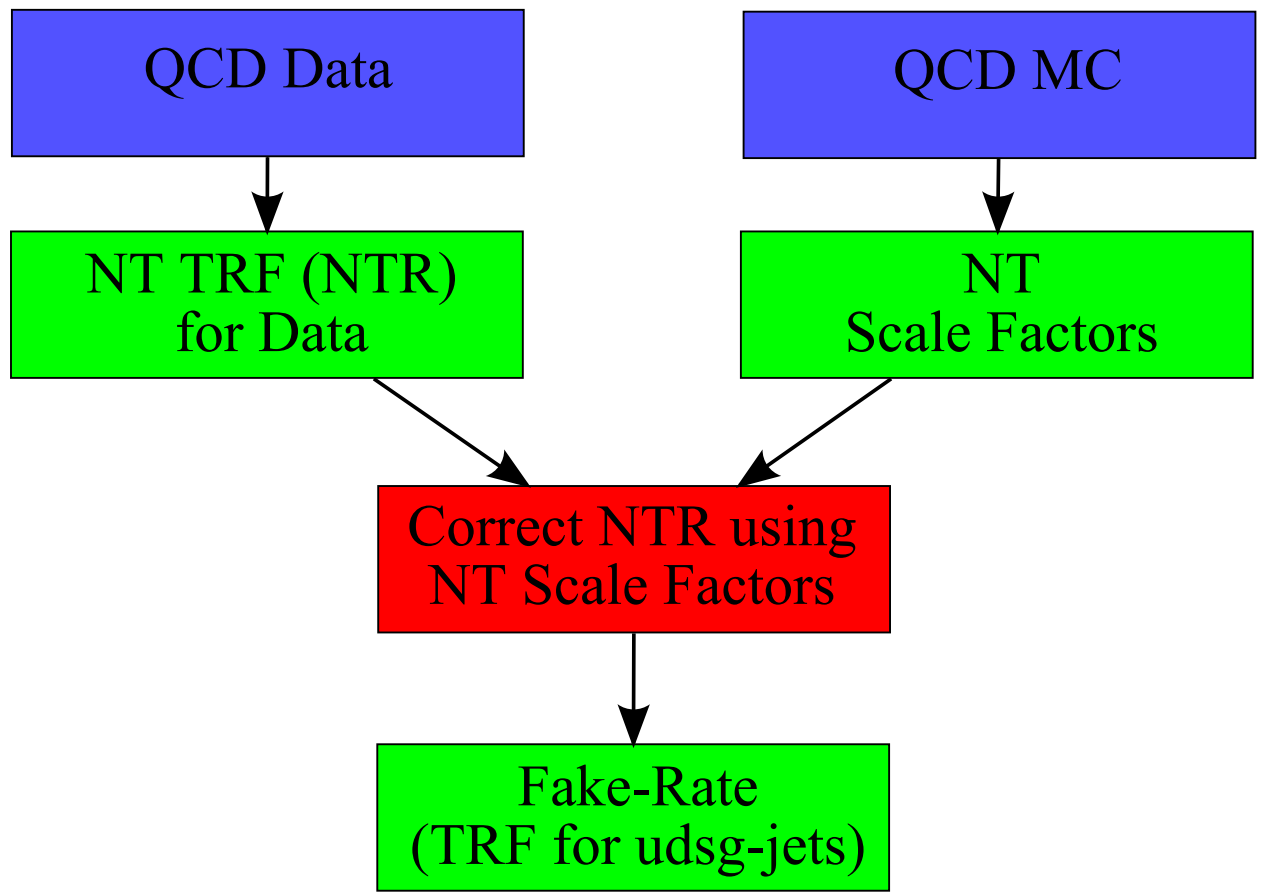

Figure 4.28: The process used to measure the fake rate in the DØcertification.

The measurement of the NTR is outlined in Section 4.7.1, the NT scale factors in Section 4.7.2, the fake rate in Section 4.7.3 and the errors on the fake rate in Section 4.7.4.

\subsubsection{Negative Tag Rate}

The negative tag rate (NTR) is measured using the COMB data skim (EM and QCD combined). The NTR is parameterised in $\mathrm{p}_{T}$ in the three $\eta$ regions outlined in Section 4.1.1.4. The NTR is given in Table 4.17 and is shown for the Loose tagger in Fig. 4.29. 


\begin{tabular}{|c|c|c|c|c|c|c|}
\hline Region & L4 & L3 & L2 & Loose & Medium & Tight \\
\hline CC & $5.36 \%$ & $4.13 \%$ & $2.96 \%$ & $1.54 \%$ & $0.89 \%$ & $0.50 \%$ \\
ICR & $4.92 \%$ & $3.75 \%$ & $2.65 \%$ & $1.34 \%$ & $0.75 \%$ & $0.42 \%$ \\
EC & $4.74 \%$ & $3.58 \%$ & $2.48 \%$ & $1.18 \%$ & $0.61 \%$ & $0.30 \%$ \\
\hline
\end{tabular}

Table 4.17: NTR measured for the COMB skim, for all $\mathrm{p}_{T}$ in the three calorimeter regions.

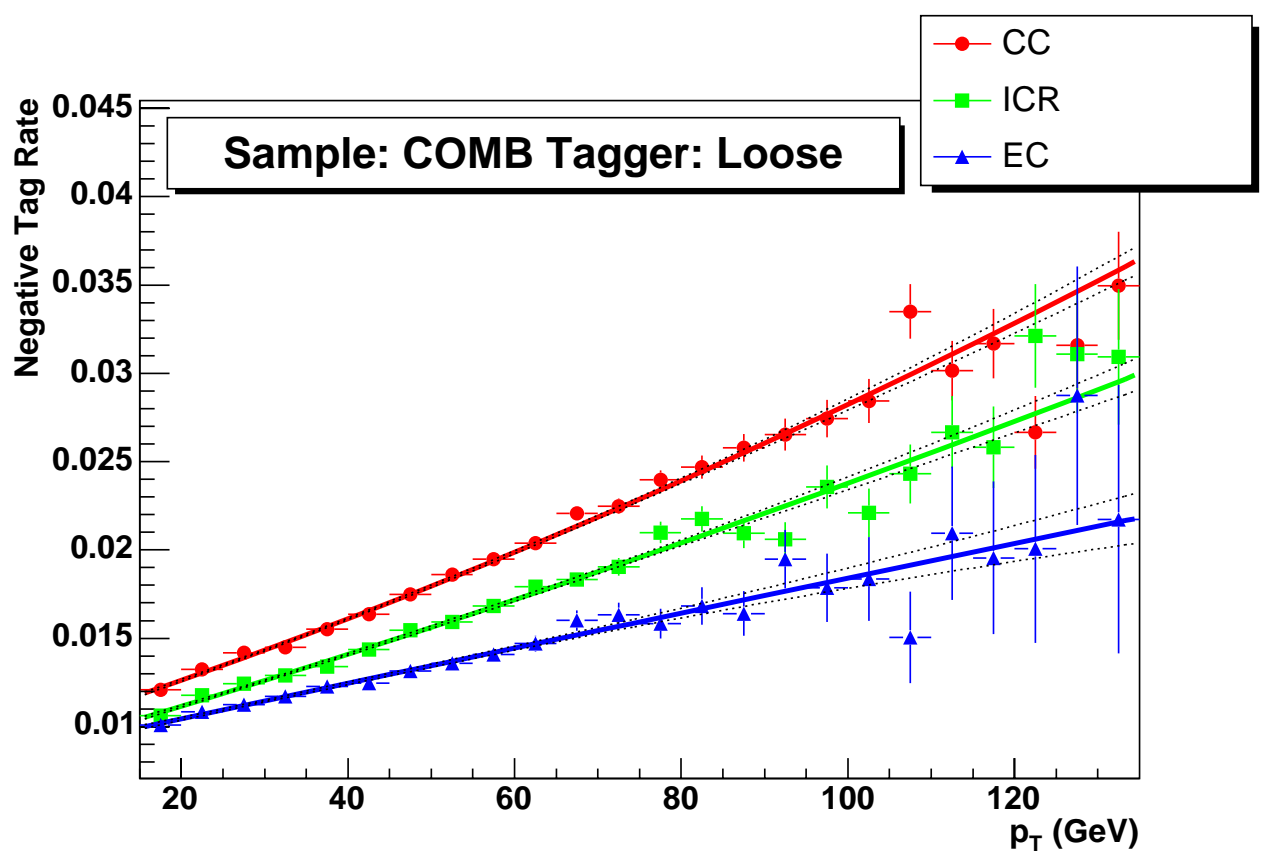

Figure 4.29: The NTR parameterisation for the COMB skim in the CC (red circles), ICR (green squares) and EC (blue triangles). The NTR is parameterised with a second order polynomial and the dotted lines show the $\pm 1 \sigma$ fit error. 


\subsubsection{Negative Tag Rate Scale Factors}

The NTR is not a perfect approximation for the fake rate, as a contribution exists in the NTR from $c$ and $b$-quarks and an asymmetry exists between the NTs and PTs due to long lived light particles. To correct the NTR to the fake rate, two NT correction scale factors (NTSF) are applied.

$S F_{h f}$ - The ratio of the light jet NTR to the total NTR. This corrects for the heavy flavour component in the NTR.

$S F_{l l}$ - The ratio between the light jet PTR and the light jet NTR. This corrects for the long lifetime fake decays not found in the NTR.

Applying these two correction factors to the NTR yields the data FTR:

$$
\mathrm{FTR}=S F_{h f} \times S F_{l l} \times \mathrm{NTR} .
$$

The NTSFs as a function of $\mathrm{p}_{T}$ in the three $\eta$ regions are shown in Fig. 4.30 for the L4 operating point measured on a MC sample consisting of all the different $\mathrm{p}_{T}$ QCD samples outlined in Table 4.4. The fit error on the NTSFs are scaled up by the $\sqrt{\chi^{2} / N D F}$ of the fit to account for the uncertainty in the fit parameterisations.

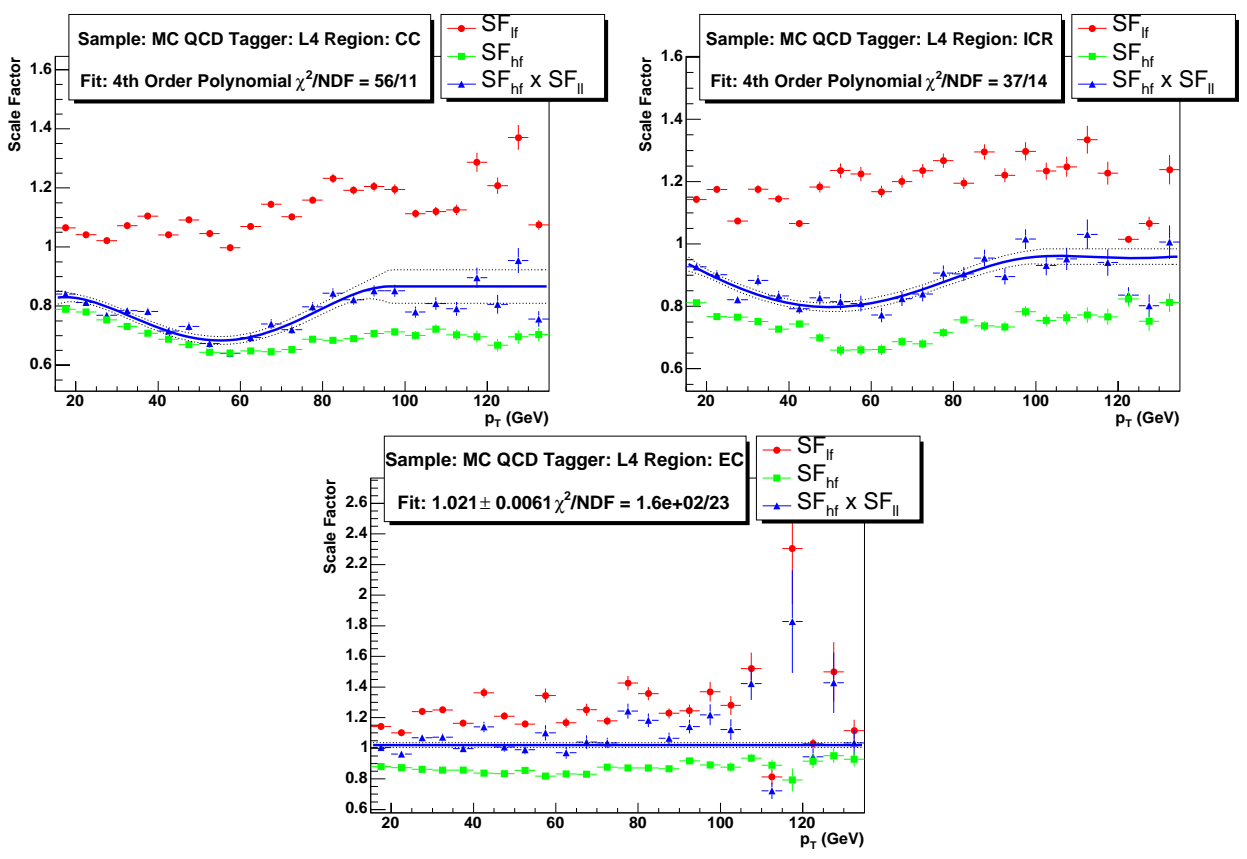

Figure 4.30: The heavy flavour correction, $S F_{h f}$ (red circles), light jet asymmetry correction, $S F_{l l}$ (green squares), and total NT SF correction (blue triangles) in the CC (top left), ICR (top right) and EC regions (bottom) for the L4 operating point. For fit details see the text. 
In the EC a constant fit is used. However, in the ICR and CC regions the NTSF is observed to be constant at high $\mathrm{p}_{T}$ but with a dip at medium $\mathrm{p}_{T}$. Several fit functions were tested to try and model this function. Fitting a straight line fit at high $\mathrm{p}_{T}$ and a parabola at low $\mathrm{p}_{T}$ was tested; however this produced discontinuities in both the value of the NTSF and the gradient. To overcome these problems a fourth order polynomial was used between $15<p_{T}<95 \mathrm{GeV}$ in the $\mathrm{CC}$ region and $15<p_{T}<135 \mathrm{GeV}$ in the ICR region. These ranges include the beginning of the constant region at high $\mathrm{p}_{T}$ so that the fit reproduces the dip and finds a maximum at the beginning of the constant region. The fit value and error at the maximum can then be used to continue the curve as a constant value. This method ensures a FTR which is a continuous function in both value and gradient and which correctly describes the constant at high $\mathrm{p}_{T}$.

\subsubsection{Fake Rate}

The FTR parameterisations and rate for the COMB skim are shown in Fig. 4.31 and Table 4.18 respectively.

\begin{tabular}{|c|c|c|c|c|c|c|}
\hline Region & L4 & L3 & L2 & Loose & Medium & Tight \\
\hline CC & $4.13 \%$ & $3.09 \%$ & $2.14 \%$ & $1.03 \%$ & $0.57 \%$ & $0.29 \%$ \\
ICR & $4.22 \%$ & $3.14 \%$ & $2.17 \%$ & $1.06 \%$ & $0.56 \%$ & $0.29 \%$ \\
EC & $4.86 \%$ & $3.62 \%$ & $2.49 \%$ & $1.19 \%$ & $0.63 \%$ & $0.30 \%$ \\
\hline
\end{tabular}

Table 4.18: The data FTR calculated on the COMB skim, in the three calorimeter regions for all $\mathrm{p}_{T}$.

\subsubsection{Systematic Uncertainties}

Systematic uncertainties exist due to: differences in the NTRs for the EM and QCD skims, the parameterisation of the NTRs into three $\eta$ regions and the uncertainty in the determination of the NTSFs. The calculation of these uncertainties is outlined below, along with a determination of the total statistical and systematic uncertainties on the fake rate.

\subsubsection{Sample Dependency Systematic Error}

The COMB skim consists of two component data samples, the QCD and EM skims. A systematic uncertainty exists due to the difference in the NTRs for these two 


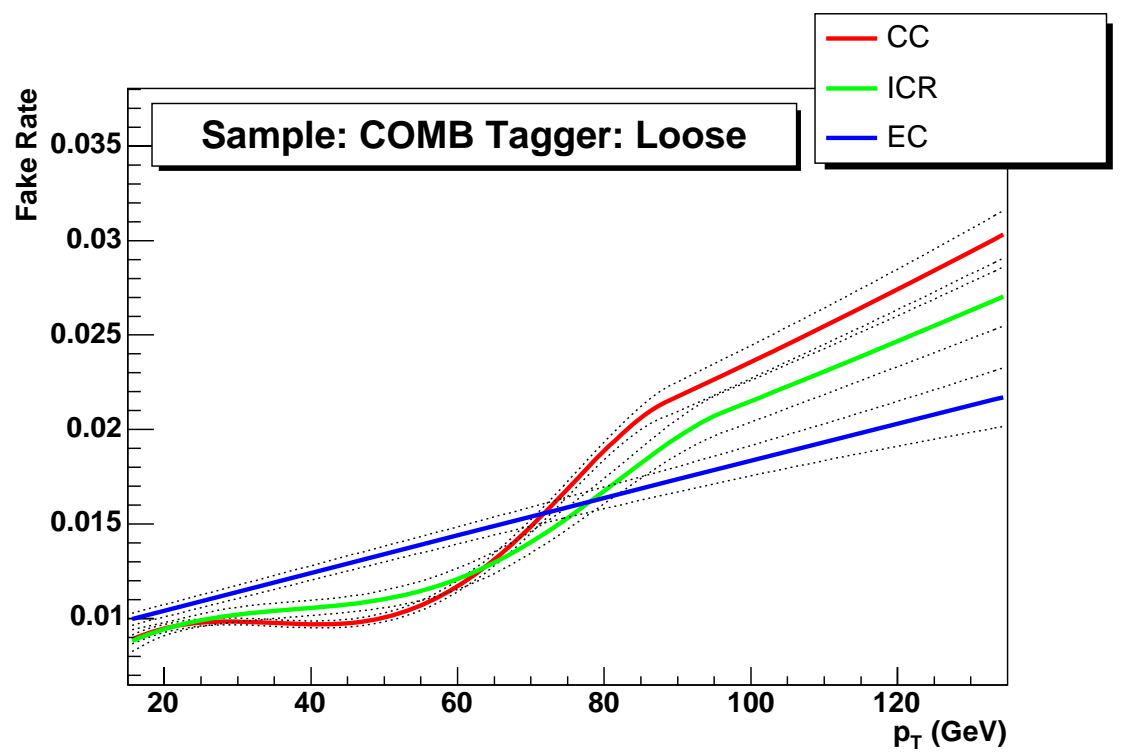

Figure 4.31: The FTRs parameterised in the CC (red), ICR (green) and EC (blue)for the Loose operating point. The dotted black lines represent the fit error.

samples. The NTRs in the EM and QCD skims, along with their ratio in the CC, ICR and EC calorimeter regions are shown in Fig. 4.32.

A small discrepancy exists between the NTRs in the QCD and EM skims, although the observed differences are much smaller than those for the JLIP and SVT taggers $[76,77]$. In the $\mathrm{CC}$ and ICR regions the differences are less than $1 \%$, although in the EC region a difference of $4 \%$ exists. Numerous studies have been conducted into the NTR differences between the EM and QCD skims [76, 77]. Several possible explanations have been suggested, although no single reason has been shown to be the cause. The two most important conditions are the trigger conditions and the skimming criteria, both of which can cause biases towards different types of jets with differing tagging rates. The SVT and JLIP taggers obtained improved agreement between the EM and QCD skims by only using jets with $\mathrm{EMF}^{10}<0.8$ ('low EMF'). However, repeating this cut for the NN tagger made the agreement worse.

A systematic error was estimated due to the differences between the QCD and EM skims. The systematic error is calculated from a constant fit to the EM and QCD ratio. Half the difference between the fit value and unity is taken as the systematic error, or if the ratio is consistent with unity within the fit error scaled by $\sqrt{\chi^{2} / N D F}$, then the scaled fit error is taken as the error. The systematic errors are shown in Table 4.19 when using all jets and in Table 4.20 when comparing only

\footnotetext{
${ }^{10}$ The fraction of the energy deposited in the EM layers of the calorimeter .
} 

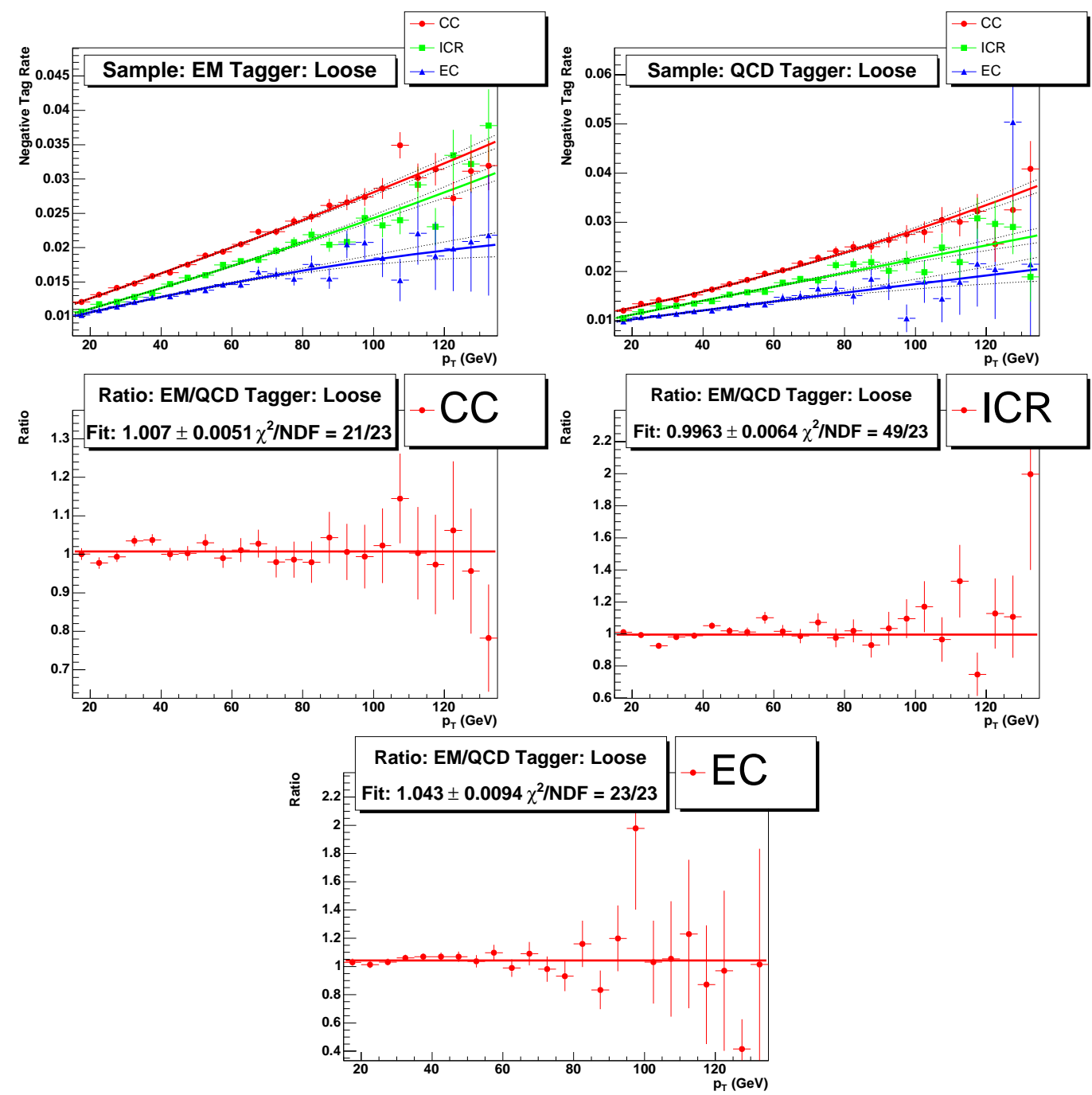

Figure 4.32: The NTR parameterisation for the EM skim (top left) and the QCD skim (top right) in the CC (red circles), ICR (green squares) and EC (blue triangles). The NTR is parameterised with a second order polynomial and the dotted lines show the $\pm 1 \sigma$ fit error. The ratio of the EM and QCD NTRs in the CC (middle left), ICR (middle right) and EC (bottom) regions is also shown. 
'low EMF' jets. The comparison which showed the largest discrepancy, in this case the 'low EMF' comparison, is used as the uncertainty.

\begin{tabular}{|c|c|c|c|c|c|c|}
\hline Region & L4 & L3 & L2 & Loose & Medium & Tight \\
\hline CC & $0.46 \%$ & $0.42 \%$ & $0.51 \%$ & $0.36 \%$ & $0.34 \%$ & $0.58 \%$ \\
ICR & $0.34 \%$ & $0.35 \%$ & $0.39 \%$ & $0.47 \%$ & $0.52 \%$ & $0.72 \%$ \\
EC & $1.81 \%$ & $1.87 \%$ & $1.96 \%$ & $2.13 \%$ & $1.77 \%$ & $1.20 \%$ \\
\hline
\end{tabular}

Table 4.19: Errors assigned to the difference in the NTRs for the EM and QCD skims..

\begin{tabular}{|c|c|c|c|c|c|c|}
\hline Region & L4 & L3 & L2 & Loose & Medium & Tight \\
\hline CC & $3.90 \%$ & $4.23 \%$ & $4.92 \%$ & $5.78 \%$ & $6.70 \%$ & $8.00 \%$ \\
ICR & $3.14 \%$ & $3.53 \%$ & $3.93 \%$ & $4.53 \%$ & $5.58 \%$ & $6.84 \%$ \\
EC & $2.89 \%$ & $2.92 \%$ & $3.16 \%$ & $3.68 \%$ & $3.34 \%$ & $3.27 \%$ \\
\hline
\end{tabular}

Table 4.20: Errors assigned to the difference in the NTRs for the EM and QCD skims for jets with $\mathrm{EMF}<0.8$.

\subsubsection{Parameterisation Systematic Error}

The uncertainty caused by the parameterisation of the NTR in the three $\eta$ regions is estimated by comparing the number of tags found by the tagger and the number predicted from the NTR. Figure 4.33 shows the actual and the predicted number of tags as a function of $\mathrm{p}_{T}$ in each of the $\eta$ regions for the COMB data sample. A systematic error due to the parameterisation of the NTR is calculated from the straight line fit to the ratio of the actual and predicted number of tags. The error is taken as the deviation of the ratio from 1 , or if the ratio is consistent with 1 within the error scaled by $\sqrt{\chi^{2} / N D F}$, then the error is taken to be the scaled fit error. The systematic errors are shown in Table 4.21 .

As a cross check the actual and predicted number of tags found in the QCD and EM skims when using the COMB parameterisation were compared. The differences were found to be small, $<1 \%$ except for the EC, and were well within the errors assigned to account for the discrepancies between the skims (shown in Table 4.20).

\subsubsection{Negative Tag Rate Scale Factors Errors}

Correcting the NTR with the NTSFs introduces a systematic error due to the uncertainty in the proportions of $b$ and $c$ in the Pythia MC simulation. A systematic 

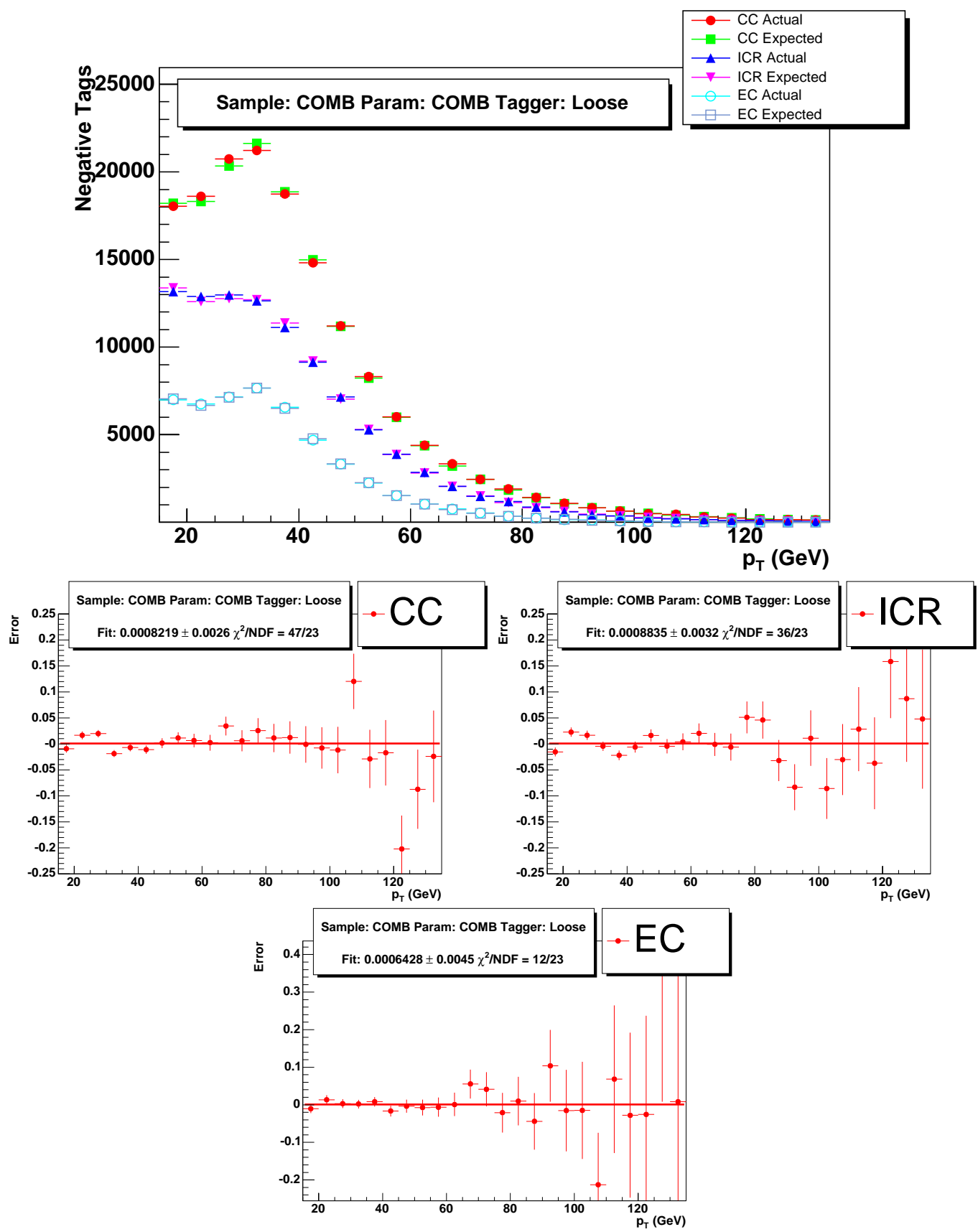

Figure 4.33: The predicted and actual number of NTs in the three eta regions for the Loose tagger on the COMB skim (top) and the relative difference between the actual and predicted number of NTs in the CC (middle left), ICR (middle right) and EC (bottom) $\eta$ regions. 


\begin{tabular}{|c|c|c|c|c|c|c|}
\hline Region & L4 & L3 & L2 & Loose & Medium & Tight \\
\hline CC & $0.14 \%$ & $0.16 \%$ & $0.19 \%$ & $0.26 \%$ & $0.34 \%$ & $0.45 \%$ \\
ICR & $0.17 \%$ & $0.20 \%$ & $0.23 \%$ & $0.32 \%$ & $0.43 \%$ & $0.58 \%$ \\
EC & $0.23 \%$ & $0.26 \%$ & $0.31 \%$ & $0.07 \%$ & $0.62 \%$ & $0.88 \%$ \\
\hline
\end{tabular}

Table 4.21: The systematic uncertainties on the COMB skim parameterisation.

error is estimated by varying the number of $b$ and $c$ quarks present in the MC by $\pm 20 \%$ [48]. The fake rate was recalculated and the largest discrepancy in the fake rate taken as the systematic error on the $b / c$ content. The errors are outlined in Tables 4.22 and 4.23 .

\begin{tabular}{|c|c|c|c|c|c|c|}
\hline Region & L4 & L3 & L2 & Loose & Medium & Tight \\
\hline CC & $3.11 \%$ & $3.49 \%$ & $3.97 \%$ & $5.01 \%$ & $5.93 \%$ & $7.21 \%$ \\
ICR & $2.88 \%$ & $3.26 \%$ & $3.75 \%$ & $4.84 \%$ & $5.69 \%$ & $6.89 \%$ \\
EC & $1.32 \%$ & $1.51 \%$ & $1.59 \%$ & $2.13 \%$ & $2.78 \%$ & $3.41 \%$ \\
\hline
\end{tabular}

Table 4.22: The systematic error in the FTR due to the uncertainty in the $b$-content in the MC.

\begin{tabular}{|c|c|c|c|c|c|c|}
\hline Region & L4 & L3 & L2 & Loose & Medium & Tight \\
\hline CC & $2.48 \%$ & $2.64 \%$ & $2.83 \%$ & $3.00 \%$ & $3.10 \%$ & $3.08 \%$ \\
ICR & $2.28 \%$ & $2.38 \%$ & $2.56 \%$ & $2.80 \%$ & $2.99 \%$ & $3.08 \%$ \\
EC & $1.30 \%$ & $1.40 \%$ & $1.49 \%$ & $1.67 \%$ & $1.98 \%$ & $2.18 \%$ \\
\hline
\end{tabular}

Table 4.23: The systematic error in the FTR due to the uncertainty in the $c$-content in the MC.

\subsubsection{Total Systematics}

The total systematic error for the FTR is calculated by adding the systematic uncertainties on the $b$ and $c$ content (as they are likely to be highly correlated) and then adding this in quadrature with the parameterisation and EM/QCD ratio errors. The total systematics are shown in Table 4.24 .

\subsubsection{Total Errors}

The total errors on the fake rate, given by the statistical (shown in Fig. 4.31) and systematic errors combined in quadrature are shown in Fig. 4.34. The dominant 


\begin{tabular}{|c|c|c|c|c|c|c|}
\hline Region & L4 & L3 & L2 & Loose & Medium & Tight \\
\hline CC & $6.81 \%$ & $7.43 \%$ & $8.37 \%$ & $9.83 \%$ & $11.10 \%$ & $12.90 \%$ \\
ICR & $6.02 \%$ & $6.64 \%$ & $7.38 \%$ & $8.87 \%$ & $10.30 \%$ & $11.90 \%$ \\
EC & $3.88 \%$ & $4.08 \%$ & $4.3 \%$ & $5.16 \%$ & $5.74 \%$ & $6.24 \%$ \\
\hline
\end{tabular}

Table 4.24: Total systematic errors on the FTR.

error is the systematic error although the statistical error on the fits has an increasing contribution as the operating point becomes tighter, and at high and low $\mathrm{p}_{T}$ values.

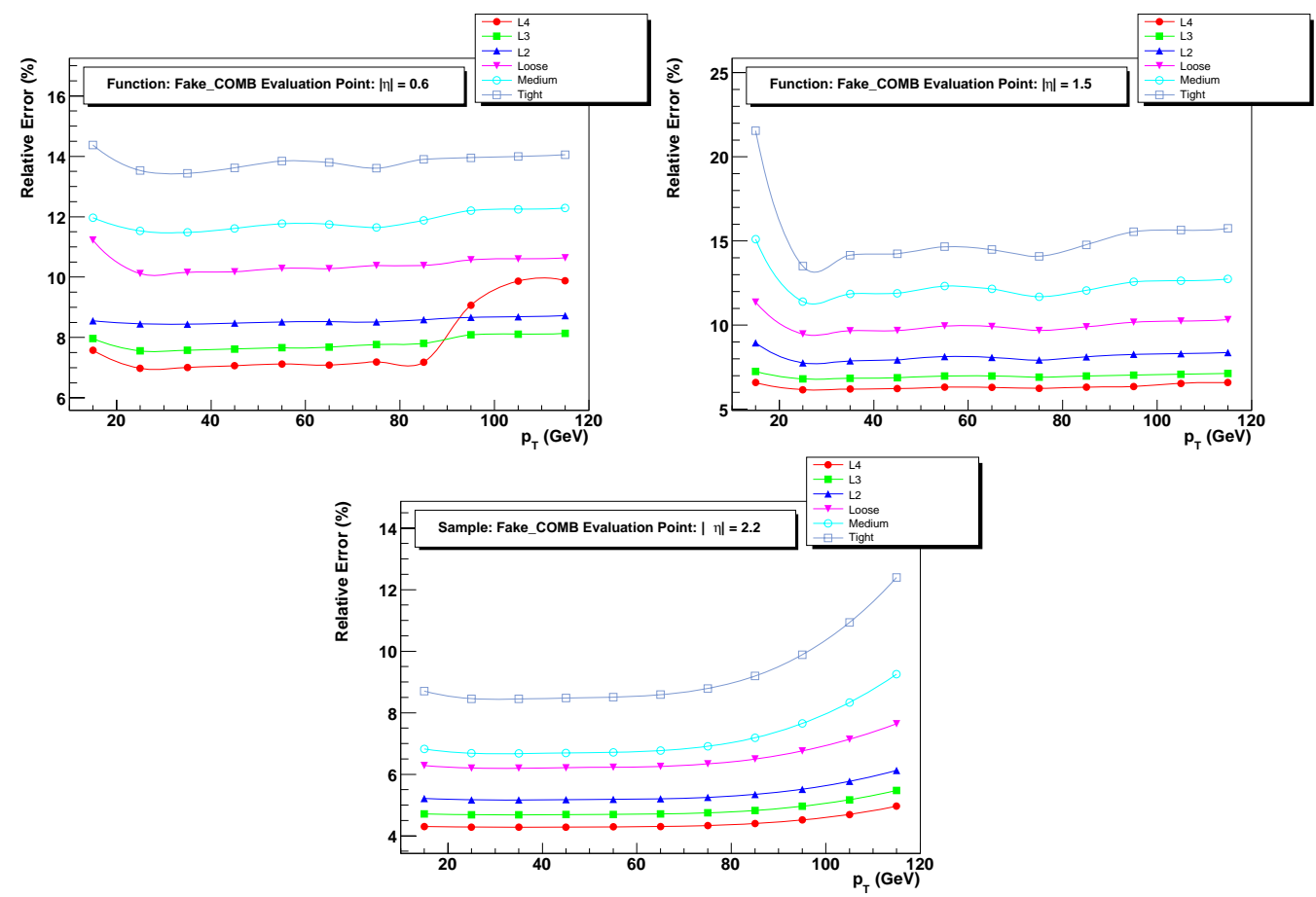

Figure 4.34: The total relative error (combined systematic and statistical) for the L4 (red circles), L3 (green squares), L2 (blue triangles), Loose (purple triangles), Medium (cyan empty circles) and Tight (grey empty squares) NN operating points on the Fake-Tag Rate in the CC (top left), ICR (top right) and $\mathrm{EC}$ (bottom) as a function of $\mathrm{p}_{T}$. The error on the $\mathrm{L} 4$ operating point in the CC increases at large $\mathrm{p}_{T}$ due to the uncertainty in the NTSF fits.

\subsection{Data Performance}

Using the $b$-efficiencies and fake rates measured in the previous two sections, it is now possible to construct a profile of the performance of the NN b-tagger on data and accurately compare it to other $b$-tagging tools. The performance of the NN and JLIP taggers measured on data, including full statistical and systematic errors, is 
shown in Fig. 4.35 for all jets. The NN tagger shows a significant gain in performance compared to the JLIP tagger, with relative increases in efficiency of up to $40 \%$ for a fixed fake rate. Fake rates are typically reduced to between a quarter and a third of their value for a fixed signal efficiency.

The performance of the NN tagger evaluated on a MC sample, but with the tagging performance and error calculated jet by jet using the data derived TRFs for $b, c$ and fake-jets is shown for all jets in the $Z \rightarrow b b$ and $Z \rightarrow q q \mathrm{MC}$ in Fig. 4.36. This demonstrates the overall performance and uncertainty of the NN tagger on a real physical process of interest. The NN tagger has a significantly better performance when compared to the JLIP tagger. The uncertainty on the $b$-efficiency is about half that of the JLIP tagger, although the looser operating points have a larger uncertainty on the fake rate.

\subsection{Conclusion}

DØ's first Neural Network $b$-tagging tool has been developed and certified ${ }^{11}$ for use at $\mathrm{D} \varnothing$. The performance of the NN tagger was measured and parameterised into TRFs for six operating points with fake rates ranging from $0.3 \%$ to $4.0 \%$. The performance of the NN tagger on data compared to the JLIP tagger showed considerable improvement, although the improvement was smaller than that in MC. For a fixed fake rate relative improvements in signal efficiency range from $\sim 40 \%$ for the tightest operating to $\sim 15 \%$ for the loosest operating point. Fake rates, for a fixed signal efficiency, are typically reduced to between a quarter and a third of their value. The improved tagging has significantly increased the sensitivity of the Higgs search at $\mathrm{D} \varnothing$. The benefit from the new tagger is expected to be the equivalent of doubling the luminosity on a double $b$-tag analysis.

\footnotetext{
${ }^{11}$ Measured, tested and approved for use on data after being reviewed by the DØ collaboration.
} 


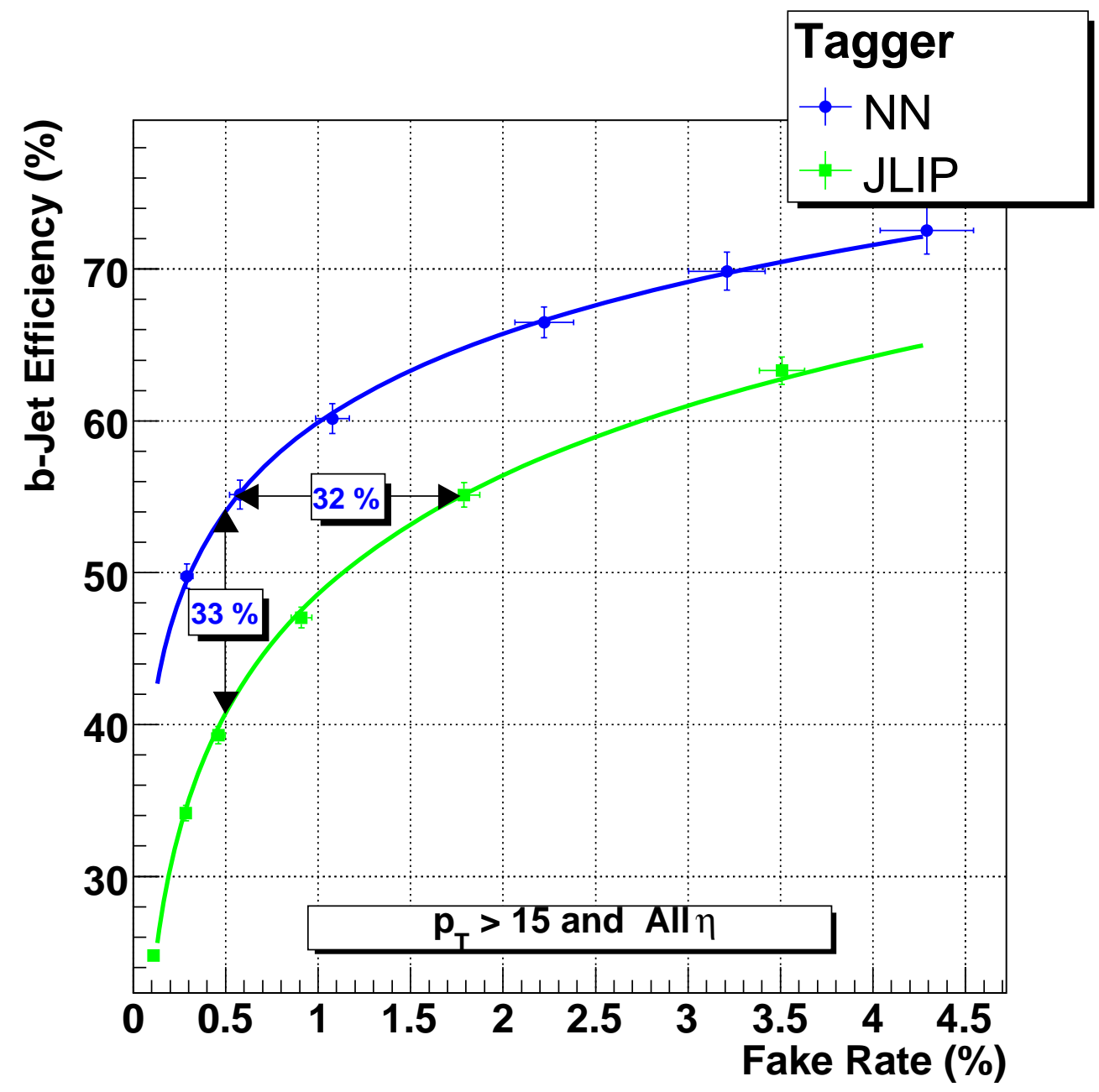

Figure 4.35: Performance profile of the NN (blue circles) and JLIP (green squares) taggers on the $b$-ID and COMB skim. The errors represent the total uncertainty, statistical and systematic. The NN tagger shows large performance gains over the JLIP tagger, with relative increases in efficiency of up to $50 \%$ for a fixed fake rate. Fake rates are typically reduced to between a quarter and a third of their value for a fixed signal efficiency. 


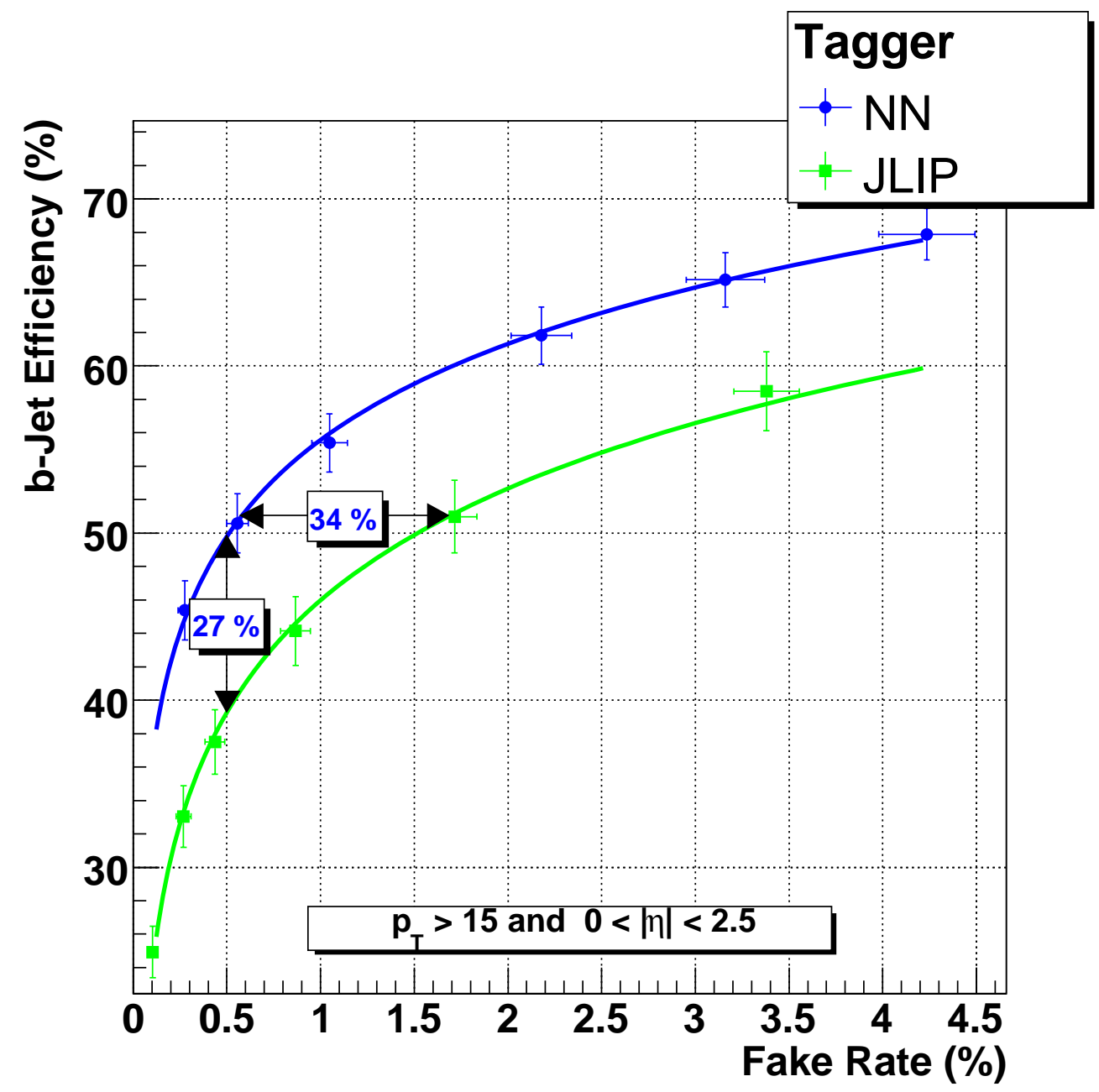

Figure 4.36: 'Data' performance profile of the NN (blue circles) and JLIP (green squares) taggers on $Z \rightarrow b \bar{b}$ and $Z \rightarrow q \bar{q}$ MC samples. The performance is calculated on a jet by jet basis. The error represents the full statistical and systematic error. 


\section{Chapter 5}

\section{Search for Neutral Supersymmetric Higgs Bosons}

\subsection{Introduction}

The largest production cross section for the SM Higgs at the Tevatron, as shown in Fig. 5.1, is $g g \rightarrow H$. However, a low mass Higgs will decay into a pair of $b$ quarks $\sim 90 \%$ of the time, as shown in Fig. 5.2. This results in the signal becoming indistinguishable from the overwhelming $g g, q \bar{q} \rightarrow b \bar{b}$ background.

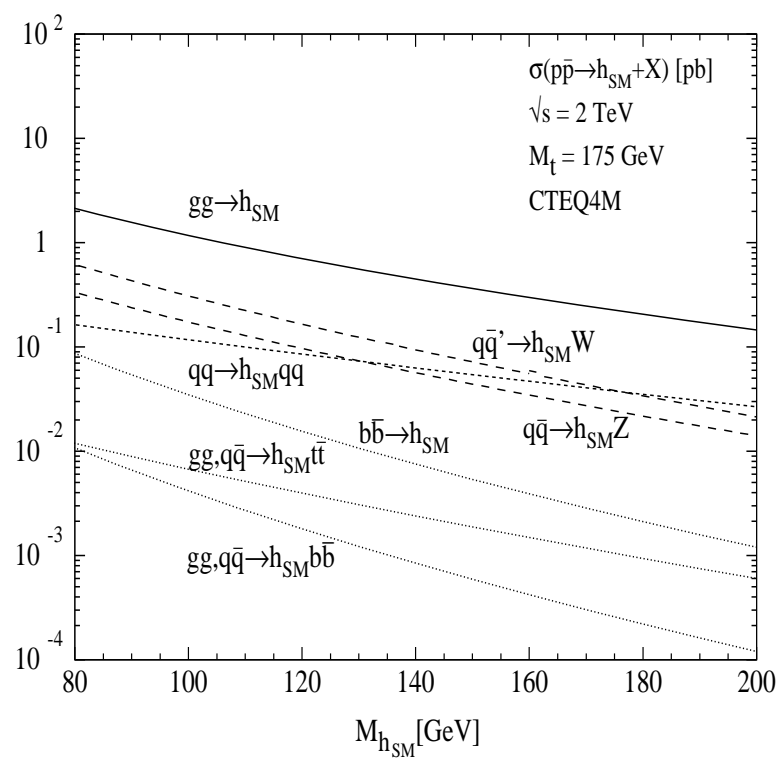

Figure 5.1: The production cross section [88] for the SM Higgs Boson at the Tevatron.

Higgs searches are therefore carried out in associated production channels. Such channels tend to have a more distinctive signature with correspondingly smaller 


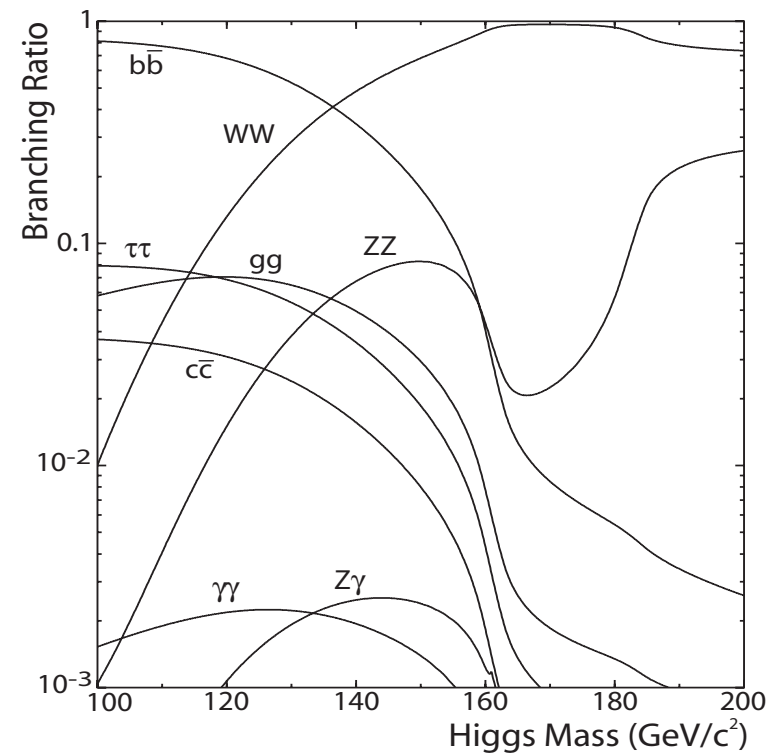

Figure 5.2: The branching ratio [88] for the SM Higgs Boson.

backgrounds. Even with their lower production cross sections they are more sensitive search channels. The associated production channels with the largest cross sections are $W H$ and $Z H$, both of which are around an order of magnitude lower than $g g \rightarrow H$.

The associated production of a Higgs with a bottom quark $(b H)$ allows suppression of backgrounds due to the presence of an additional $b$-quark. It has a production cross section a factor of 2 smaller, whilst $b b H$ production is an order of magnitude less (than the $W H$ and $Z H$ channels). Additionally the sensitivity of these channels is orders of magnitudes smaller due to the prohibitively high multi-jet background. However, there are several Higgs schemes where the coupling of the Higgs to the $b$ is enhanced and consequently $b H$ production becomes the single largest source of Higgs bosons. Searches in the $b H / b b H$ channel allow such theories to be investigated. The Minimal Supersymmetric extension of the Standard Model (MSSM) is such a scheme, where the coupling of the Higgs to the bottom-quark is enhanced by a factor of $\tan \beta$, and therefore the production cross section is enhanced by $\tan ^{2} \beta$ (see Section 1.2.1).

There are two major search channels in the MSSM scheme $h b \rightarrow b b b$ and $h b \rightarrow$ $b \tau \tau$ (where $\mathrm{h}=\mathrm{A}, \mathrm{h}$ and $\mathrm{H}$ ) which correspond to the two largest branching ratios for a low mass Higgs. This chapter documents three iterations of the $h b \rightarrow b b b$ search channel, which follows past searches at CDF in Run I [22] and at D $\varnothing$ in Run II [23]. The next section gives a brief overview of this analysis and the strategy used. 


\subsubsection{Analysis Overview}

The tree level processes which contribute to the associated production of a neutral MSSM Higgs boson with a b-quark, when the Higgs decays to two $b$-jets, are shown in Fig. 5.3 and outlined below:

- $q \bar{q}, \mathrm{gg} \rightarrow b \bar{b} \mathrm{~h} \rightarrow b \bar{b} b \bar{b}$

- $q \bar{q}, \mathrm{gg} \rightarrow \mathrm{bg} \rightarrow \mathrm{hb} \rightarrow \mathrm{b} b \bar{b}$
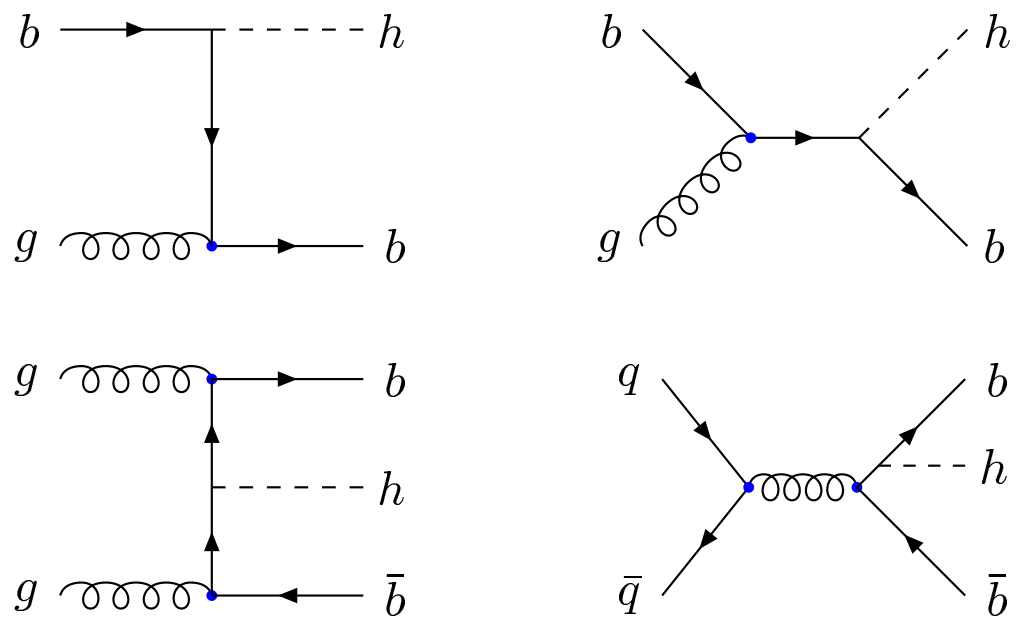

Figure 5.3: Leading-Order Feynman diagrams showing gb $\rightarrow \mathrm{hb}$ and gg, $q \bar{q} \rightarrow b \bar{b} \mathrm{~h}$ production.

The signal is selected by requiring three $b$-jets. The main backgrounds are QCD production of heavy flavour jets such as $b b j j$ ( $j$ represents $u d s g$ here), $b b b b$ and $b b c c$. The uncertainty on the Monte Carlo production of such processes can only be calculated to LO at the moment, and the associated uncertainty on the cross section is up to 100\% (see Section 5.3.2). These large errors mean that the simulation is unsuitable for use in the background prediction. A method was therefore developed to predict the background distributions from data.

The triple tagged background distributions are predicted from data in three stages. Firstly, a tag rate function (TRF) for jets is measured using the data sample. Secondly, the TRF is used to predict the shape of the triple tagged background distributions by weighting the double tagged events by the probability that the non-b-tagged jets in the event would be tagged according to the TRF. Finally, the predicted shape of the triple $b$-tagged background distributions is normalised to data outside the signal region. A flowchart outlining the background prediction method is shown in Fig. 5.4. 
The di-jet mass distributions from the data background prediction, the signal simulation and the data are then used to set limits on the production of the neutral MSSM Higgs bosons.

\subsubsection{The Three Analysis Generations}

The author has participated in the three versions of the $h b$ analysis described in this chapter. The first analysis documented in Section 5.5 is the 'p14 Pass1' [71] analysis which was approved by the Collaboration and published in Physical Review Letters [24]. The author carried out trigger studies for this analysis.

The second analysis, documented in Section 5.6, is the 'p14 Pass2' analysis. The aim of the Pass2 analysis was to investigate the effect of the higher performance $b$-tagging from the Neural Network tagger in the $h b$ analysis and was not aimed at external publication. The author undertook all the work in this version of the analysis, and co-developed a new analysis method and background model ${ }^{1}$ which was needed due to the improved $b$-tagging.

The above analyses are jointly referred to as the p14 analyses due to the version of the offline reconstruction code used.

The last analysis, documented in Section 5.7, is the 'p17' analysis. The author's contribution included the design of the triggers (see Section 3.4) which collected two thirds of the data, the joint development of the new background model and analysis method, and the Neural Network $b$-tagging. This version of the analysis has been approved as a preliminary result by the collaboration for ICHEP 2006 [26], and is aiming for publication in early 2007.

The data used in the analyses are outlined in Section 5.2, the Monte Carlo samples in Section 5.3 and the triggers in Section 5.4.

\subsection{Data}

The Pass1 and Pass2 p14 analyses were conducted on data collected from November 2002 - June 2004 and the p17 analysis was conducted on data collected from November 2002 - April 2006. Three 'versions' of the data were used in the analyses, referred to as 'Pass1', 'Pass2' and 'p17'.

Pass1 consists of physics objects reconstructed from the raw data. Pass2 data had calorimeter fixes for known problems and $t 42^{2}$ cell killing applied before the

\footnotetext{
${ }^{1}$ This work was jointly carried out with members of the Saclay group.

${ }^{2}$ The suppression of calorimeter cells whose energy is less than $2 \sigma$ above noise, unless they have a neighbouring cell whose energy is $4 \sigma$ above noise.
} 


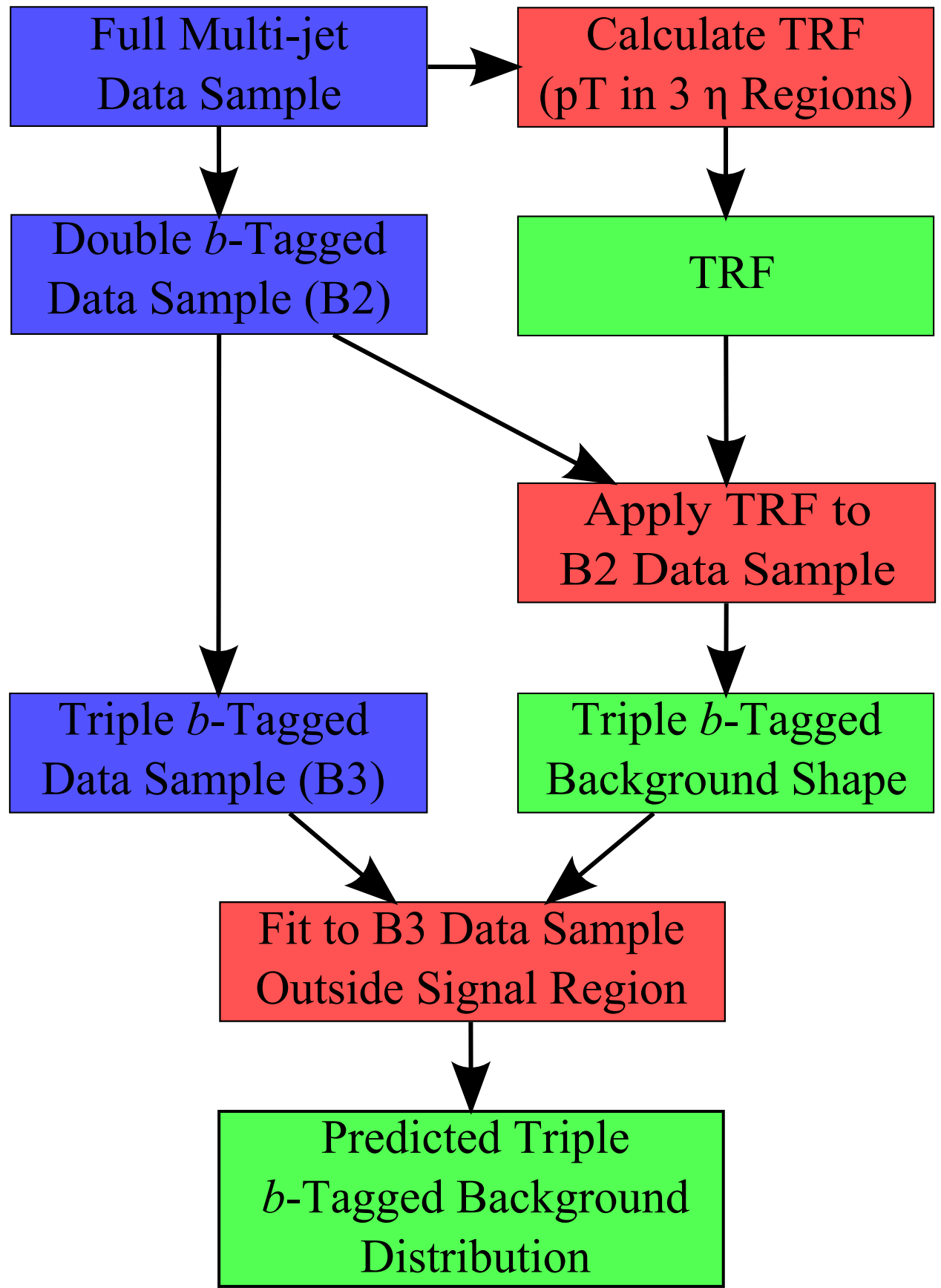

Figure 5.4: The $h b$ analysis triple $b$-tagged background prediction method. The different coloured boxes correspond to: data samples (blue), derived samples (green) and processes (red). 
reconstruction of the physics objects [89]. p17 data included full hadronic and EM calorimeter calibrations and the jet reconstruction threshold was lowered from $\mathrm{p}_{T}>8 \mathrm{GeV}$ to $\mathrm{p}_{T}>6 \mathrm{GeV}$, in addition to the Pass2 improvements.

\subsubsection{Skimming}

Suitable data were 'skimmed' from the full data set; events were required to have three jets with uncorrected transverse momentum $\mathrm{p}_{T}>15 \mathrm{GeV}$ with two having $\mathrm{p}_{T}>25 \mathrm{GeV}$. The $\mathrm{p}_{T}$ and $\eta$ distributions of the jets in the Pass1, Pass2 and p17 skims are shown in Fig. 5.5. The p17 data has a much smoother $\eta$ distribution due to the calorimeter and jet data quality improvements.

\subsubsection{Data Quality}

All runs flagged as having detector problems in either the tracking (SMT and CFT), calorimeter $(\mathrm{Cal})$ or muon systems were removed. This resulted in the loss of $14-$ $15 \%$ of the data as outlined in Table 5.1. All events with bad luminosity information were also excluded, resulting in the loss of $<1 \%$ of the data.

\begin{tabular}{|c|c|c|c|c|c|c|}
\hline Epoch & Luminosity $\left(\mathrm{pb}^{-1}\right)$ & SMT & CFT & Muon & Cal & Any \\
\hline Pre-v12 & 122 & $6.7 \%$ & $1.5 \%$ & $9.4 \%$ & $9.0 \%$ & $24.6 \%$ \\
v12 & 219 & $0.5 \%$ & $0.2 \%$ & $3.6 \%$ & $5.4 \%$ & $9.4 \%$ \\
\hline All pre-v13 & 341 & $2.7 \%$ & $0.7 \%$ & $5.7 \%$ & $6.7 \%$ & $14.8 \%$ \\
\hline v13 & 379 & $2.8 \%$ & $1.1 \%$ & $1.5 \%$ & $7.3 \%$ & $12.2 \%$ \\
v14 & 324 & $2.6 \%$ & $1.2 \%$ & $2.0 \%$ & $10.7 \%$ & $16.1 \%$ \\
\hline All & 1044 & $2.7 \%$ & $1.0 \%$ & $3.0 \%$ & $8.2 \%$ & $14.3 \%$ \\
\hline
\end{tabular}

Table 5.1: The percentage of runs in the different trigger list epochs which are classed as bad in the SMT, CFT, Muon, Cal and Any detector subsystems [90]. The 'All pre-v13' epoch corresponds to the p14 analyses and the 'All' epoch corresponds to the p17 analysis.

\subsection{Event Simulation}

Due to differences between MC and data, all simulated events have their jet resolution [91], jet taggability [45], jet reconstruction efficiency [92] and b-tagging [74] corrected to match those seen in data. The signal and background MC samples are outlined in more detail in the following two sections. 

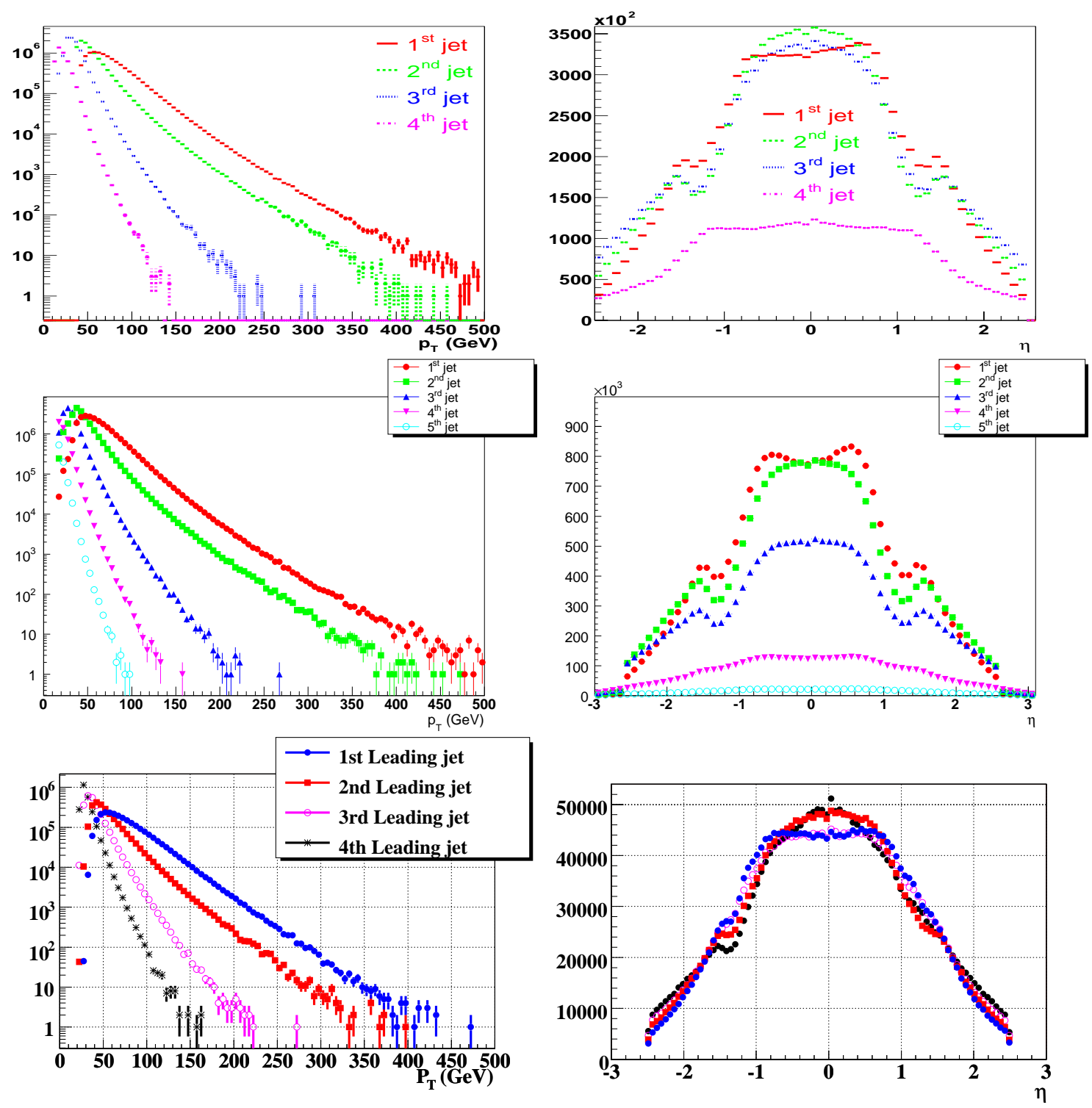

Figure 5.5: The $\mathrm{p}_{T}$ (left) and $\eta$ distribution (right) of the jets in the Pass1 (top), Pass2 (middle) and $\mathrm{p} 17$ data sets from the highest $\mathrm{p}_{T}$ (1st jet) to the lowest $\mathrm{p}_{T}$ jet (4th or 5 th). The jets have passed the skimming, trigger, data quality and taggability requirements. 


\subsubsection{Signal Samples}

Due to radiative effects the two $h b$ production processes, outlined in Section 5.1.1, are nearly indistinguishable in the 3/4-jet channels [93, 94]. Kinematic comparisons of the two processes using Pythia [70] samples produced with initial state radiation (ISR) and final state radiation (FSR) turned on demonstrated that the two samples have almost identical kinematic distributions [95]. Thus to avoid double counting of events, only one of the processes is used to model the signal. The $h b$ process was chosen as it has a simpler NLO calculation with smaller scale uncertainties.

The $h b \mathrm{MC}$ is produced using Pythia with ISR and FSR, a hard scatter of $\mathrm{p}_{T}>15 \mathrm{GeV}$ and no rapidity cuts. For the p14 analyses 100,000 events were produced for each of the following Higgs masses: 90, 100, 110, 120, 130, $150 \mathrm{GeV}$. For the p17 analysis $~ 50,000$ events were produced for Higgs masses of 100, 110, 120, 150 and $170 \mathrm{GeV}$.

The Pythia events were compared to those from the NLO calculation from MCFM [96]. The events were weighted so that the $\mathrm{p}_{T}$ and $\eta$ spectrum of the Higgs matched the NLO spectrum predicted by MCFM. Cross sections were calculated using the NLO calculations from MCFM and are shown in Table 5.2. The renormalisation and factorisation scales $(\mu)$ used in the MCFM simulation were fixed to the same value of $\mu=\left(2 m_{b}+M_{h}\right) / 4$ motivated by the studies in [95, 97]. The cross sections used in the p14 and p17 analyses had $\mathrm{a} \sim 15 \%$ difference in the NLO values due to different versions of the CTEQ PDFs [98] being used in MCFM. The p14 analysis used CTEQ5 and p17 CTEQ6. A larger error was used in the p17 analysis to reflect this difference.

The jet $\mathrm{p}_{T}$ and $\eta$ distributions are shown in Fig. 5.6 for the Pass2 signal sample as a representative distribution.
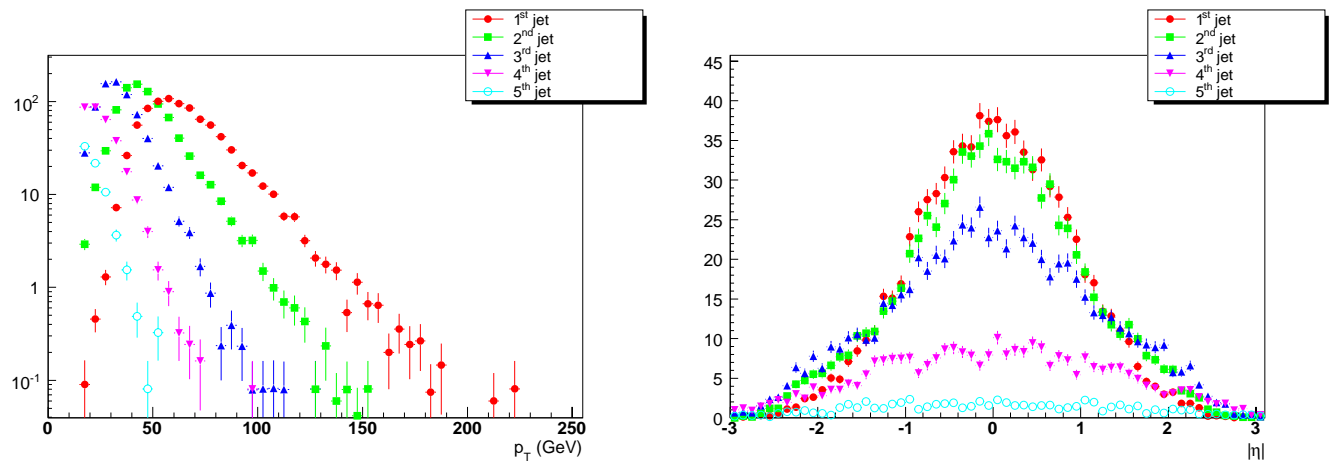

Figure 5.6: $\mathrm{p}_{T}$ (left) and $\eta$ distribution (right) for the Pass $2 \mathrm{MC}$ samples from the highest $\mathrm{p}_{T}$ (1st jet) to the lowest $\mathrm{p}_{T}$ jet (5th). The jets have passed the skimming, trigger, quality and taggability requirements. 


\begin{tabular}{|c|c|c|}
\hline \multirow{2}{*}{$m_{h}(\mathrm{GeV})$} & \multicolumn{2}{|c|}{$\mathrm{hb}(\mathrm{fb})\left(\mathrm{p}_{T}>15 \mathrm{GeV}\right)$} \\
\cline { 2 - 3 } & $\mathrm{p} 14$ & $\mathrm{p} 17$ \\
\hline 90 & 9.99 & 10.93 \\
100 & 6.45 & 7.28 \\
110 & 4.42 & 5.00 \\
120 & 3.03 & 3.52 \\
130 & 2.16 & $n / a$ \\
150 & 1.12 & 1.29 \\
170 & $n / a$ & 0.71 \\
\hline
\end{tabular}

Table 5.2: Signal production cross sections used to normalise the $h b \mathrm{MC}$ samples. The values are taken from the NLO calculations and correspond to $\tan \beta$ of 1 [96].

\subsubsection{Combined Cross Section}

At large $\tan \beta$ the combined production cross section of the $\mathrm{h}$ and $\mathrm{H}$ is nearly always equal to that of the A boson. As this analysis does not distinguish between the three neutral Higgs bosons, the total cross section is therefore assumed to be double that of the A alone. The production cross sections of the h, $\mathrm{H}$ and $\mathrm{A}$ bosons in association with a bottom quark as a function of $m_{A}$, calculated using the HQQ program [88], are shown in Fig. 5.7.

The effect of any possible mass splitting between the Higgs bosons away from the degenerate region was checked by modelling the three Higgs bosons as separate distributions at different masses. The effect that this had on the final limit was less than $1 \%[71]$.

\subsubsection{Width}

The widths of the neutral Higgs bosons vary as a function of $\tan \beta$ and mass as shown in Fig. 5.8. For the majority of the phase space to which this analysis is sensitive, the Higgs width is less than the detector di-jet mass resolution $(\sim 20 \mathrm{GeV})$ and so could be neglected. However, at high masses and large $\tan \beta$, the width of the Higgs becomes greater than the detector di-jet mass resolution. The effect a large Higgs width has on the analysis was studied and found to be small. It was concluded that the Higgs width could be neglected [71].

\subsubsection{Background Samples}

Any process which produces a multi-jet final state is a potential background to this channel. The main backgrounds are listed below. 


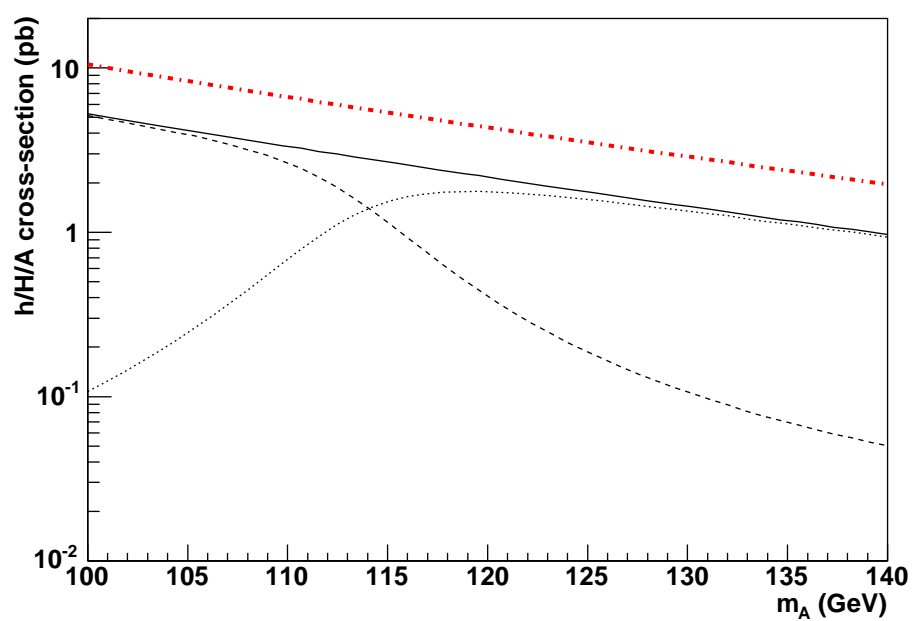

Figure 5.7: Production cross sections for neutral Higgs bosons associated with a $b \bar{b}$ pair at leading-order as a function of $m_{A}$ only, for $\tan \beta$ of 30 calculated using HQQ [88]. The A is solid, the $\mathrm{h}$ is dashed, and the $\mathrm{H}$ is dotted. The sum of $\mathrm{h}, \mathrm{H}$, and A production is shown dashed-dotted, and is always equal to twice the A production alone.

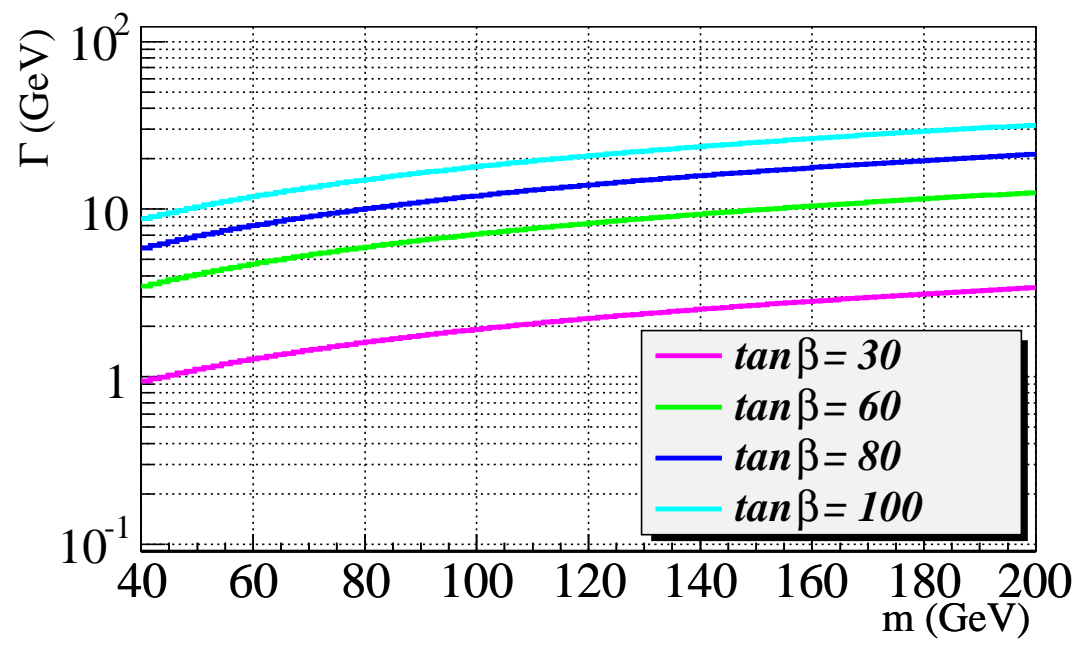

Figure 5.8: Higgs width as a function of mass and $\tan \beta$. At high $\tan \beta$ and mass the width is larger than the experimental resolution of $\sim 20 \mathrm{GeV}$. 
jjj/jjjj - Multi-jet QCD production with 3 or more fake-jets (uds or gluon-jets) in the final state. This sample was modelled and normalised using the data sample, and is referred to as $j j j$.

$b b j / b b j j$ - Multi-jet QCD production with 3 or more jets in the final state, two of which must be $b$-jets with the remaining jets udsc or gluon-jets. Alpgen [85], a leading-order matrix element generator, is used to generate the parton level events. The parton level events are then passed through Pythia to model the partonic cascades and their transformation into their final observable hadron states. The $b b j$ and $b b j j$ samples were merged together, with the $b b j j$ sample weighted by 0.85 so that the combined $b b j$ and $b b j j$ samples matched the jet multiplicity in data. The combined sample is referred to as $b b j$.

$b b b / b b b b$ - Multi-jet QCD production with 3 or $4 b$-jets in the final state, again modelled using Alpgen.

$t t$ - Always has at least $2 b$-jets from the decay of the $t$-quarks, and will often have 2 or 4 more jets depending on how the $\mathrm{W}$ bosons decay. Generated using Pythia, with the production cross section taken from the combined D $\varnothing$ Run II measurement [99].

$Z j-Z$ decays into $2 b$-jets with an additional $u d s c$ or gluon-jet. This is expected to be a small contribution to the background. Generated using Pythia with the cross section given by the DØ Run II measurement [100].

$Z b / Z b b-Z$ decays into $2 b$-jets with 1 or 2 additional $b$-jets. Generated using Pythia with the cross section given by MADGRAPH $[101,102]$.

The background MC samples along with their cross sections and generator level cuts are outlined in Table 5.3. The p17 cross sections are higher due to a couple of changes. The PDF set was altered from CTEQ5L to CTEQ6L1, which increased the yields by $\sim 10 \%$. In addition the renormalisation and factorisation scale $\left(Q^{2}\right)$ in ALPGEN was switched from $Q^{2}=1 / N \sum p_{t}^{2}$ to $Q^{2}=\sum p_{t}^{2}$, where $N$ is the number of partons. This resulted in a $50 \%$ decrease in the cross sections. As there is no reason to choose one of these scales over the other, the uncertainty on the cross section for these processes is very large. In addition the requirements on the $b \bar{b} b \bar{b}$ sample were loosened to the asymmetrical cut of $2 b$-quarks with $\mathrm{p}_{T}>25 \mathrm{GeV}$ and $3 b$-quarks with $\mathrm{p}_{T}>25 \mathrm{GeV}$. This is to account for the three $b$ background which was overlooked in the p14 Pass1 analysis. 


\begin{tabular}{|c|c|c|}
\hline Process & Cross-section (pb) & Generator Cuts ( $\mathrm{p}_{T}$ in $\left.\mathrm{GeV}\right)$ \\
\hline$b \bar{b} \mathrm{jj}$ & 3810 (ALPGEN p14) & \multirow{2}{*}{$\mathrm{p}_{T}(b)>25, \mathrm{p}_{T}(j)>15,|\eta|<3.0$} \\
\hline$b \bar{b} \mathrm{jj}$ & 2540 (ALPGEN p17) & \\
\hline$b \bar{b} \mathrm{j}$ & 8940 (ALPGEN p14) & \multirow[t]{2}{*}{ " } \\
\hline$b \bar{b} \mathbf{j}$ & 3810 (ALPGEN p17) & \\
\hline$b \bar{b} b \bar{b}$ & 58 (ALPGEN p14) & \multirow{3}{*}{$\begin{array}{c}\mathrm{p}_{T}(b)>15,|\eta|<3.0 \\
\mathrm{p}_{T}(2 b)>25, \mathrm{p}_{T}(3 b)>15 \mathrm{GeV},|\eta|<3.0 \\
\mathrm{p}_{T}(2 b)>25, \mathrm{p}_{T}(3 b)>15 \mathrm{GeV},|\eta|<3.0\end{array}$} \\
\hline$b \bar{b} b \bar{b}$ & 120 (ALPGEN p17) & \\
\hline$b \bar{b} b$ & $3.2($ ALPGEN p17) & \\
\hline$\overline{t \bar{t}}$ & $7.1(\mathrm{D} \varnothing$ Run I) & \multirow{4}{*}{$\begin{array}{c}\text { none } \\
\mathrm{p}_{T}(\mathrm{~b})>15,|\eta|<3.0\end{array}$} \\
\hline $\mathrm{Z}(b \bar{b})+$ jets & 1180 (DØ Run II) & \\
\hline $\mathrm{Zb}$ & 10 (MADGRAPH) & \\
\hline $\mathrm{Z} b \bar{b}$ & 3 (MADGRAPH) & \\
\hline
\end{tabular}

Table 5.3: Background MC cross sections, source of the cross section and generator level cuts where applicable.

\section{$5.4 \quad$ Trigger}

Due to the large cross section for QCD multi-jet production the data are collected with a specialised multi-jet trigger. The triggers are designed to maximise signal acceptance whilst maintaining a reasonable rate to tape of $\sim 3 \mathrm{~Hz}$.

The data were collected with five different generations of the multi-jet trigger referred to as versions v9/10, v11, v12, v13 and v14. The triggers became increasingly sophisticated as greater rejection was required at higher instantaneous luminosities. Full details of all the triggers can be found in Section 3.4. The integrated luminosity collected by each of the triggers after data quality cuts is shown in Table 5.4. The total luminosity recorded by the triggers is less than that for all triggers due to data quality cuts and pre-scales ${ }^{3}$.

The multi-jet triggers are outlined in detail in Section 3.4.

\subsubsection{Trigger Simulation}

The use of triggers to collect data leads to biases in the kinematic quantities of the events. MC events, being simulated and therefore not collected using a trigger, need to have such trigger effects simulated to properly model the data collected for the analysis.

\footnotetext{
${ }^{3}$ Random selection of a subset of the triggered events to be written out to tape so that the rate remains within bandwidth constraints at high luminosities.
} 


\begin{tabular}{|c|c|c|c|c|}
\hline \multirow{2}{*}{ Trigger List Version } & \multirow{2}{*}{ Name } & \multicolumn{3}{|c|}{ Integrated Luminosity $\left(\mathrm{pb}^{-1}\right)$} \\
\cline { 3 - 5 } & & Pass1 & Pass2 & $\mathrm{p} 17$ \\
\hline v9/v10 & 3JT15 & 33.4 & 36.1 & 28.8 \\
v11 & 3JT15_PV & 54.8 & 57.6 & 55.4 \\
v12 & 3JT15_2J25_PVZ & 172.0 & 185.3 & 171.9 \\
v13 & JT2_3JT15L_IP_VX & $n / a$ & $n / a$ & 328.3 \\
v14 & JT2_3JT15L_IP_VX & $n / a$ & $n / a$ & 171.3 \\
\hline Total & & 261.2 & 279.0 & 880.1 \\
\hline
\end{tabular}

Table 5.4: The recorded integrated luminosity of each of the multi-jet triggers after data quality cuts for the Pass1, Pass2 and p17 analyses.

The effect of the trigger is simulated in the $\mathrm{MC}$ by weighting each event by the probability that it would have passed the trigger. The trigger simulation calculates the probability that each offline jet is associated with a L1 calorimeter jet tower, L2 jet and L3 jet based upon the $\mathrm{p}_{T}$ and $\eta$ of the jet $[71,103]$. The individual jet probabilities are then combined into an overall event probability.

The L3 $b$-tagging requirement, used in the v13 and v14 triggers, is simulated by modelling a L3 IP probability distribution for jets based upon the flavour of the jets, and if the jet is tagged offline or not. L3 IP probabilities are then randomly selected from the distributions for each jet in an event, and combined into an overall simulated event probability. The turn-on curve for the simulated event probability was measured on data relative to events with a L3 IP probability of $<0.05^{4}$. The probability of the event passing the trigger is then directly obtained from the turn-on curve [26].

Each MC event is weighted by the probability that it has passed one version of the trigger. MC events have a trigger version chosen by random selection based upon the integrated luminosities collected by each trigger.

\subsubsection{Trigger Efficiencies}

The trigger efficiencies calculated for each of the signal MC samples are shown in Table 5.5 for the jet terms only, in Table 5.6 for the L3 IP $b$-tag term only and in Table 5.7 for the combined trigger terms. The efficiencies are quoted relative to the basic p17 analysis cuts of $\mathrm{p}_{T}>40,25$, and $15 \mathrm{GeV}$ for the first, second, and third leading jets respectively. When the L3 IP b-tag cut is included in the trigger simulation, the efficiencies are calculated relative to events which have at least three Tight NN b-tags.

\footnotetext{
${ }^{4} \mathrm{An}$ IP probability of $<0.05$ is the $b$-tagging applied in the trigger condition.
} 


\begin{tabular}{|c|c|c|c|c|c|}
\hline \multirow{2}{*}{ Trigger List } & \multicolumn{5}{|c|}{$m_{h}(\mathrm{GeV})$} \\
\cline { 2 - 6 } & 100 & 110 & 120 & 150 & 170 \\
\hline v9/10 & $55.7 \pm 0.6 \%$ & $56.9 \pm 0.5 \%$ & $60.1 \pm 0.5 \%$ & $59.5 \pm 0.5 \%$ & $63.9 \pm 0.4 \%$ \\
v11 & $42.6 \pm 0.5 \%$ & $45.2 \pm 0.5 \%$ & $48.6 \pm 0.5 \%$ & $49.5 \pm 0.4 \%$ & $55.7 \pm 0.4 \%$ \\
v12 & $42.6 \pm 0.5 \%$ & $45.2 \pm 0.5 \%$ & $48.6 \pm 0.5 \%$ & $49.5 \pm 0.4 \%$ & $55.7 \pm 0.4 \%$ \\
v13 & $45.5 \pm 0.5 \%$ & $47.7 \pm 0.5 \%$ & $51.7 \pm 0.5 \%$ & $52.6 \pm 0.4 \%$ & $58.2 \pm 0.4 \%$ \\
v14 & $47.7 \pm 0.6 \%$ & $48.9 \pm 0.5 \%$ & $52.3 \pm 0.5 \%$ & $53.4 \pm 0.4 \%$ & $58.9 \pm 0.4 \%$ \\
\hline Overall & $46.1 \pm 0.6 \%$ & $46.9 \pm 0.5 \%$ & $51.3 \pm 0.5 \%$ & $53.0 \pm 0.4 \%$ & $58.3 \pm 0.5 \%$ \\
\hline
\end{tabular}

Table 5.5: The trigger efficiencies for the various signal masses for the jet terms only. The 'Overall' efficiency is given by the integrated luminosity weighted average efficiency of the trigger lists. The uncertainties are statistical only.

\begin{tabular}{|c|c|c|c|c|c|}
\hline \multirow{2}{*}{ Trigger List } & \multicolumn{5}{|c|}{$m_{h}(\mathrm{GeV})$} \\
\cline { 2 - 6 } & 100 & 110 & 120 & 150 & 170 \\
\hline v13 & $90 \pm 1 \%$ & $87 \pm 1 \%$ & $85 \pm 1 \%$ & $89 \pm 1 \%$ & $85 \pm 1 \%$ \\
v14 & $87 \pm 1 \%$ & $87 \pm 1 \%$ & $85 \pm 1 \%$ & $86 \pm 1 \%$ & $86 \pm 1 \%$ \\
\hline
\end{tabular}

Table 5.6: The trigger efficiencies for the various signal masses for the L3 IP $b$-tag term only. Events were required to have at least three NN tight $b$-tags. The uncertainties are statistical only.

\begin{tabular}{|c|c|c|c|c|c|}
\hline \multirow{2}{*}{ Trigger List } & \multicolumn{5}{|c|}{$m_{h}(\mathrm{GeV})$} \\
\cline { 2 - 6 } & 100 & 110 & 120 & 150 & 170 \\
\hline v9/10 & $86 \pm 2 \%$ & $85 \pm 1 \%$ & $85 \pm 1 \%$ & $84 \pm 1 \%$ & $85 \pm 1 \%$ \\
v11 & $58 \pm 2 \%$ & $62 \pm 2 \%$ & $65 \pm 2 \%$ & $70 \pm 1 \%$ & $74 \pm 1 \%$ \\
v12 & $58 \pm 2 \%$ & $62 \pm 2 \%$ & $65 \pm 2 \%$ & $70 \pm 1 \%$ & $74 \pm 1 \%$ \\
v13 & $60 \pm 2 \%$ & $59 \pm 2 \%$ & $61 \pm 1 \%$ & $63 \pm 1 \%$ & $66 \pm 1 \%$ \\
v14 & $58 \pm 2 \%$ & $62 \pm 2 \%$ & $63 \pm 2 \%$ & $63 \pm 1 \%$ & $67 \pm 1 \%$ \\
\hline Overall & $60 \pm 2 \%$ & $57 \pm 2 \%$ & $63 \pm 1 \%$ & $64 \pm 1 \%$ & $68 \pm 1 \%$ \\
\hline
\end{tabular}

Table 5.7: The trigger efficiencies for various Higgs masses, when requiring at least three NN tight $b$-tagged jets. The 'Overall' efficiency is given by the integrated luminosity weighted average efficiency of the trigger lists. The uncertainties are statistical only. 


\subsubsection{Trigger Crosschecks}

The trigger simulation described in Section 5.4.1 was compared in detail to the trigger efficiencies found using the official trigger simulation package, TrigSim [104]. The trigger efficiencies calculated using the two methods and the percentage differences between the methods are shown in Table 5.8.

The results from TrigSim are all consistently higher than the results from the analysis trigger simulation. This is expected as TrigSim is known to be over optimistic in its jet finding. These differences diminish as you go to higher Higgs masses where the jet energies are further away from the jet turn-on curves. The discrepancy between the two methods is also within the systematic error of $9 \%$ assigned to the trigger in the p14 analyses.

\begin{tabular}{|c|c|c|c|}
\hline \multirow{2}{*}{$m_{h}(\mathrm{GeV})$} & \multirow{2}{*}{ Trigger Efficiency } & Difference \\
\cline { 2 - 3 } & TrigSim & Analysis Trigger Simulation & \\
\hline 90 & $43 \pm 5 \%$ & $41 \pm 4 \%$ & $5 \%$ \\
110 & $51 \pm 4 \%$ & $48 \pm 4 \%$ & $6 \%$ \\
130 & $59 \pm 3 \%$ & $55 \pm 5 \%$ & $7 \%$ \\
150 & $61 \pm 2 \%$ & $60 \pm 5 \%$ & $2 \%$ \\
\hline
\end{tabular}

Table 5.8: Trigger efficiencies calculated using TrigSim and the analysis trigger simulation for the jet only trigger (v9 - v12) measured relative to the skimming criteria $\left(3\right.$ jets $\mathrm{p}_{T}>15 \mathrm{GeV}$ and 2 jets $\mathrm{p}_{T}>25 \mathrm{GeV}$ ). The TrigSim errors are statistical only and the errors on the analysis trigger simulation includes the $9 \%$ systematic error from the 'p14' analyses (see Section 5.5.4.1).

\subsection{Pass1 Analysis}

The analysis is split into several sections. The event selection is outlined in Section 5.5.1, the analysis method used to predict the background in Section 5.5.2, the method used to set limits in Section 5.5.3, the systematic uncertainties in Section 5.5.4 and finally the results are presented in Section 5.5.5.

\subsubsection{Event Selection}

Events were selected for inclusion in the analysis if they had:

- 3 - 5 taggable jets with $|\eta|<2.5$ and leading jet cuts of $\mathrm{p}_{T}>45,25$ and $15 \mathrm{GeV}$.

- A PV with $|z|<35 \mathrm{~cm}$. 
- Fired a $h b$ trigger.

- At least three $b$-tags.

The Loose SVT tagger operating point was used to identify $b$-jets.

\subsubsection{Analysis Method}

The method used to predict the background from data is outlined in the following sections.

\subsubsection{Double Tag Comparison}

The double tag data sample is used to normalise the $b b j \mathrm{MC}$ background and to measure the FTRF for the multi-jet sample.

The tag rate function (TRF) for all jets in the data sample is defined as:

$$
\mathrm{TRF}_{\text {all }}=\frac{\text { Number of Tagged Jets }}{\text { Number of Taggable Jets }}
$$

and is measured as a function of $\mathrm{p}_{T}$ in $3 \eta$-regions.

$\mathrm{TRF}_{\text {all }}$ is used to model the initial contribution from $j j j$ in the double tagged channel by weighting each data event by the probability that there are 2 or more 'fake' tagged jets in the event. There is a heavy flavour component in $\mathrm{TRF}_{\text {all }}$ from the heavy jets in the data sample, and there will consequentially be a heavy flavour component in the $j j j$ prediction which is corrected for later on before the final normalisation determination.

An estimate of the normalisation of the $b b j \mathrm{MC}$ can now be made using the double tagged data events. The number of double tagged $b b j \mathrm{MC}$ events $\left(N_{b b j}\right)$ is normalised to the total integral $\left(N_{\text {all }}\right)$ of the double $b$-tagged data after subtraction of the $j j j$ and the other small MC samples $(Z j, t t$ and $b b b b$ fixed by their cross sections). The initial fit of the modelled background to the double tag data sample is shown in Fig. 5.9.

The presence of the heavy flavour jets in $\mathrm{TRF}_{\text {all }}$ can be corrected for using the $b b j$ $\mathrm{MC}$ sample, assuming $\mathrm{TRF}_{\text {all }}$ is dominated by fake-tags and heavy flavour jets from $b b j$. The resulting TRF will be the multi-jet fake-tag rate function (FTRF). The FTRF can then be used to make a more accurate prediction of the $j j j$ background and consequentially a more accurate prediction of the $b b j$ normalisation. The FTRF is calculated using the following formula:

$$
\mathrm{FTRF}=\mathrm{TRF}-\frac{N_{b b j}}{N_{a l l}} \times \mathrm{TRF}_{b b j},
$$




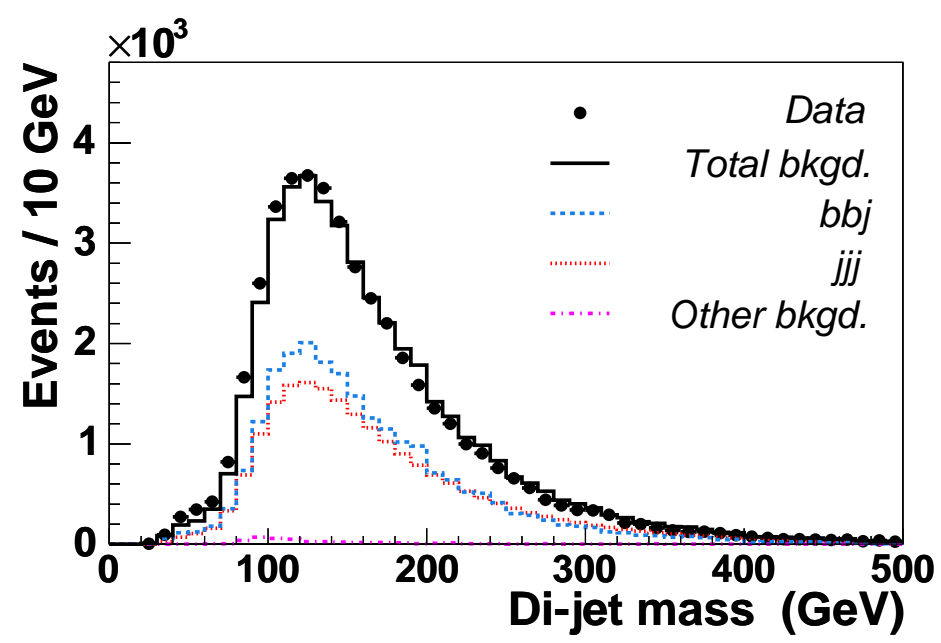

Figure 5.9: The total simulated double $b$-tagged di-jet mass distribution (black line), consisting of $j j j$ (red dotted line), $b b j$ (blue dotted line), and other small backgrounds: $Z j, t t$, and $b b b b$ (Purple dot-dashed line), to data (black dots). The $j j j$ distribution shown in this figure has a heavy flavour component which is corrected for, as explained in the text.

where $\mathrm{TRF}_{b b j}$ is the TRF derived from the $b b j \mathrm{MC}$ sample. The TRF and FTRF are shown in Fig. 5.10.

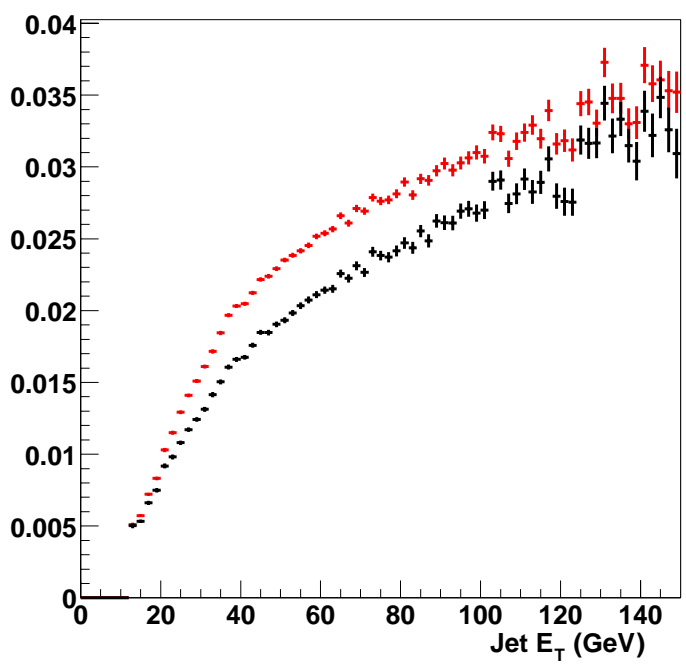

Figure 5.10: $\mathrm{TRF}_{\text {all }}$ (red points) and FTRF (black points).

The recalculated $j j j$ background prediction along with the corrected normalisation for the $b b j \mathrm{MC}$, are shown in Fig. 5.11.

\subsubsection{Triple Tag MC Background Comparison}

Using the triple tagged distributions of $j j j$ predicted from data, the data normalised bbj MC, and the additional small MC backgrounds, a comparison can be made 


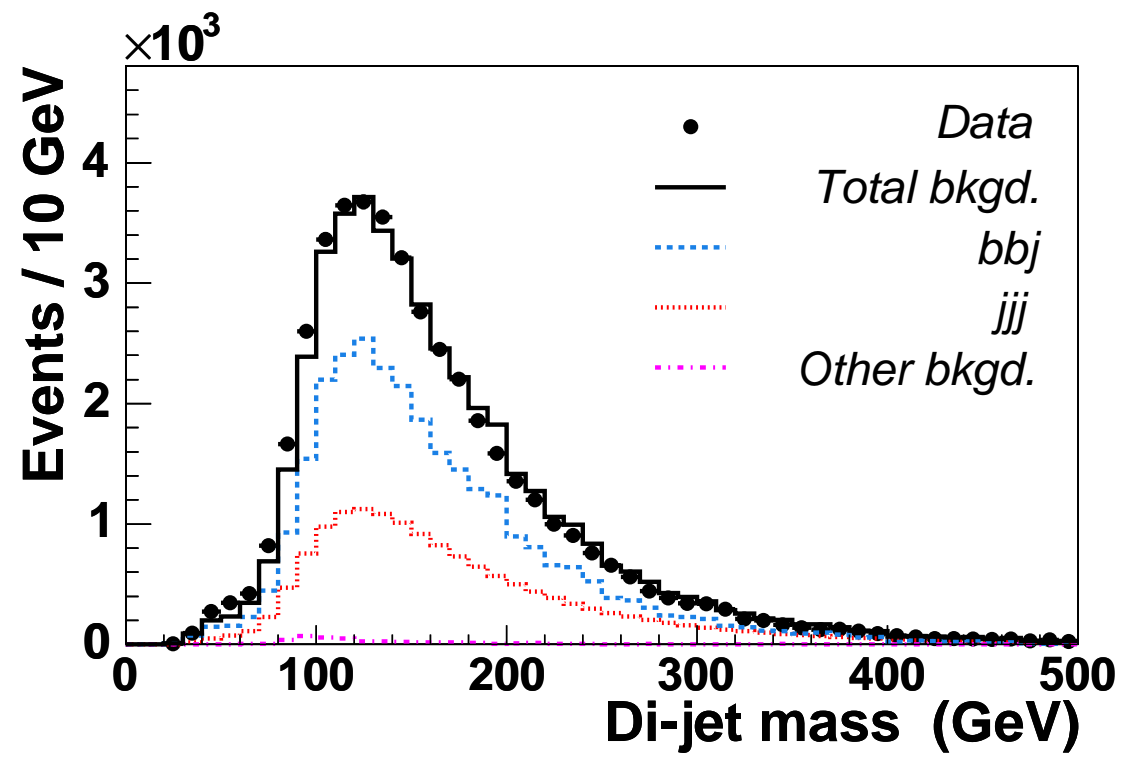

Figure 5.11: Fit of the total background (black line) consisting of $b b j$ (blue dotted line), $j j j$ (red dotted line) and other small backgrounds (purple dot-dashed line) to the double tagged data (black points) sample using the corrected FTRF.

between the triple tagged MC background prediction and data as shown in Fig. 5.12 for the Pass1 data.

\subsubsection{Triple Tag Background Prediction}

The triple tag background is predicted from data. The shape of the distribution is predicted first and then normalised to data.

The shape of the $b b j$ triple tagged background can be predicted using the FTRF and the double tagged data events. Events in the double tagged data sample are weighted, using the FTRF, by the probability that any of the jets, neglecting the two highest $\mathrm{p}_{T}$ tagged jets, are tagged. The resulting distribution provides the shape of the triple tagged background distribution.

The correct normalisation is then calculated by fitting the predicted triple tagged background distribution to the actual triple tagged data on a bin by bin basis outside the signal region $( \pm 1 \sigma$ of a Gaussian fit to the signal) using TMinuit [87]. This provides the scale factor $\left(S_{\text {Data }}\right)$ by which the predicted background is multiplied to obtain the correct normalisation.

The triple tagged data, background prediction and $h b\left(m_{h}=120 \mathrm{GeV}\right) \mathrm{MC}$ events for an illustrative $\tan \beta$ value of 100 are shown in Fig. 5.13. There is no excess, and the predicted background, MC background and data are all within agreement. 


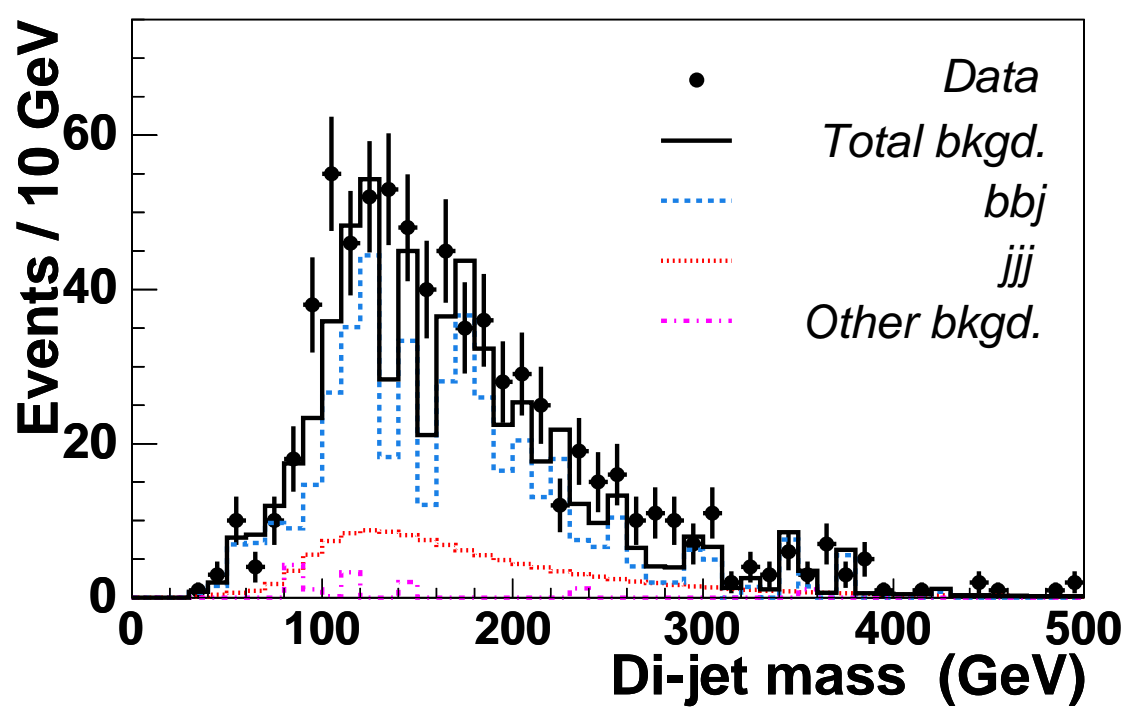

Figure 5.12: The MC predicted triple tagged background (black line) for the Loose SVT tagger, consisting of the correctly normalised MC $b b j$ sample (blue dotted line), the $j j j$ prediction from data (red dotted line) and other small MC contributions (purple dot-dashed line), compared to the triple tagged data sample (black dots).

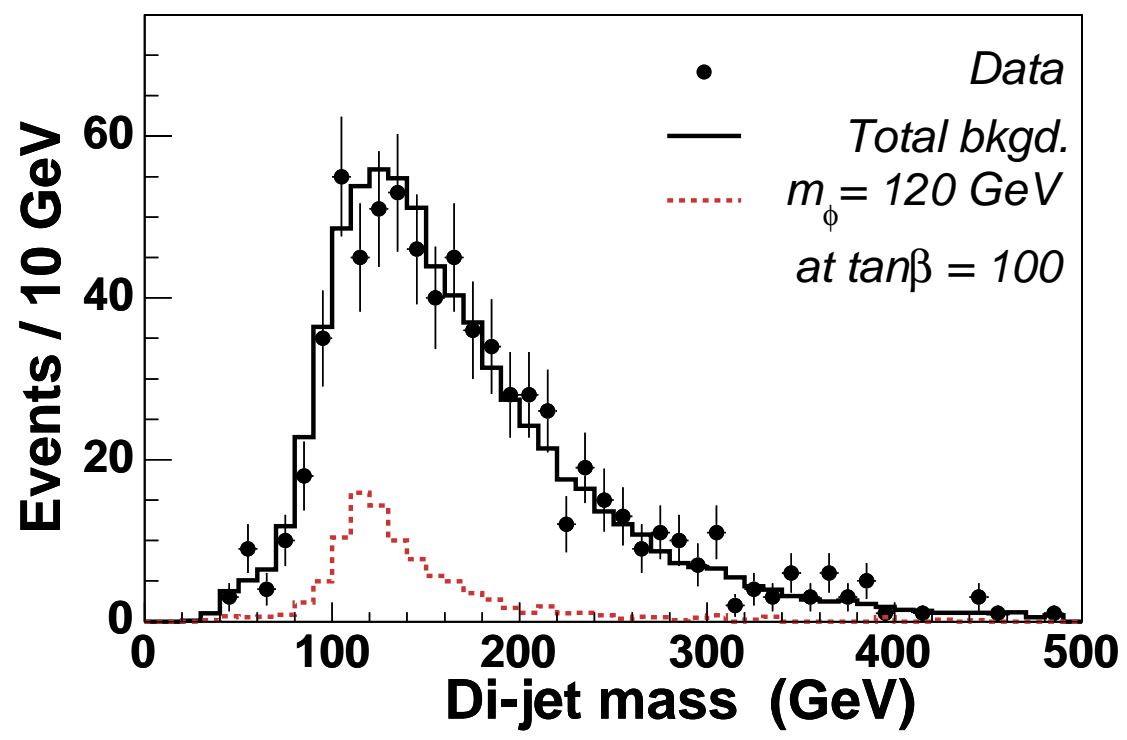

Figure 5.13: The data (points), normalised background (solid line), and the Higgs signal for $m_{A}=120 \mathrm{GeV}$ (dashed line) at $\tan \beta \simeq 100$. 


\subsubsection{Limit Setting}

Upper limits at 95\% confidence level (CL) are set on $\tan \beta$ for each Higgs mass. Limits are set using the TLimit implementation [105] of MCLimit [106]. MCLimit calculates limits on signal production using each histogram bin (of the chosen discriminating variable) as an independent counting search. Each of the separate 'search channels' are combined to provide an overall limit on signal production. The modified frequentist confidence level $\left(C L_{s}\right)$ is used to set limits:

$$
C L_{s}=\frac{C L_{s+b}}{C L_{b}}
$$

where $C L_{s+b}$ is the confidence level for the signal plus background ( $\left.\mathrm{s}+\mathrm{b}\right)$ hypothesis, and $C L_{b}$ is the confidence level for the background only hypothesis.

There are two types of limits set, expected and observed. Expected limits are calculated using the simulated background and signal distributions only, assuming no signal production, and indicate the limit setting potential of the experiment. The observed limits are calculated using the data and the simulated signal and background distributions, and is the actual limit on signal production set from the data.

Background and signal systematic uncertainties are taken into account by assuming that the numbers of signal and background events vary according to gaussian error distributions, whose widths are equal to the systematic uncertainties. The number of signal and background events in each bin is then sampled from the gaussian distributions a predetermined number of times.

Limits are set using the triple $b$-tagged di-jet mass for the signal, data and predicted background. The signal cross section is multiplied by $\tan ^{2} \beta$ until a confidence level for signal production of less than $5 \%$ is achieved.

\subsubsection{Systematic Uncertainties}

Systematic uncertainties are derived for the triple tagged MC signal and predicted background distributions.

\subsubsection{Signal Systematic Uncertainties}

The derived signal distributions have several uncertainties, which can be split into those uncertainties which are dependent on the Higgs mass, and those independent of the Higgs mass. Uncertainties which are independent of the Higgs mass are: 
Theory - The uncertainty in the simulation of the signal samples. The theoretical uncertainty due to the correction of the LO signal processes to match the NLO spectra were taken account of. This error was estimated to be half the 5\% [71] correction. Uncertainties due to the PDF set used and the choice of factorisation and normalisation scale were not included in the theoretical error.

Luminosity (Lum) - The uncertainty on the D $\varnothing$ luminosity measurement. The luminosity group has estimated an uncertainty of $6.5 \%$ [107].

Trigger (Trig) - The uncertainty in the trigger simulation. This was estimated to be $9 \%$ [71]. The uncertainty takes into account closure tests on the method, the statistics available and the quality of the fits to the trigger turn-on curves.

The following sources of systematic error vary as a function of Higgs mass. Errors are calculated by measuring the alteration in the triple tagged signal di-jet mass distribution, in terms of both the integral and shape of the distribution, as the errors are fluctuated. The unfluctuated distribution is weighted so that its integral is equal to one, this histogram is then used to weight, on a bin by bin basis, the $-1 \sigma$ and $+1 \sigma$ fluctuated histograms, with the final errors calculated using the following equation:

$$
\text { Error }=\frac{1}{2} \frac{\sqrt{\left(I_{m}-I_{u}\right)^{2}+\left(I_{p}-I_{u}\right)^{2}}}{I_{u}},
$$

where $I_{u}, I_{m}$, and $I_{p}$ are the integrals of the unfluctuated, $-1 \sigma$ fluctuated and $+1 \sigma$ fluctuated histograms respectively. The factor of a half is used to correct to the half width half maximum value. The following systematic uncertainties are evaluated as such, with the resulting errors shown in Table 5.9.

Jet Energy Resolution (JER) - MC jet energies are smeared, with an associated uncertainty, to account for the difference in resolution of jet energies between data and MC [91].

Jet Energy Scale (JES) - Uncertainty in the jet energy scale corrections [108].

Jet Reconstruction and ID (Jet-ID) - Uncertainty in the scale factor used to adjust the MC efficiency to that measured in data [92].

$b$-tagging ( $b$-ID) - Uncertainty on the $b / c$ and fake-tagging rates used to correct the MC tagging performance to that of data [109]. 


\begin{tabular}{|c|c|c|c|c|c|c|c|c|}
\hline$m_{h}(\mathrm{GeV})$ & Theory & Lum & Trig & $b$-ID & JES & JER & Jet-ID & Total \\
\hline 90 & 5.0 & 6.5 & 9.0 & 14 & 7.5 & 1.4 & 3.8 & 20.4 \\
100 & 5.0 & 6.5 & 9.0 & 15 & 7.1 & 0.7 & 3.8 & 21.0 \\
110 & 5.0 & 6.5 & 9.0 & 14 & 8.1 & 0.7 & 3.7 & 20.6 \\
120 & 5.0 & 6.5 & 9.0 & 14 & 8.5 & 0.8 & 3.6 & 21.0 \\
130 & 5.0 & 6.5 & 9.0 & 14 & 7.8 & 0.4 & 3.3 & 20.4 \\
150 & 5.0 & 6.5 & 9.0 & 15 & 7.7 & 0.8 & 3.4 & 21.1 \\
\hline
\end{tabular}

Table 5.9: The individual errors for the Loose SVT tagger from each source (in \%), added in quadrature to give the total error on the signal MC.

\subsubsection{Background Systematics Uncertainties}

There are three uncertainties considered on the background prediction, all of which are considered to be independent of the Higgs mass as the signal has very little impact on the background prediction.

Normalisation - The uncertainty associated with the normalisation of the predicted background outside the signal region. This is taken as the error on the data normalisation scale factor $\left(S_{\text {Data }}\right)$.

Shape - The uncertainty in the shape of the predicted background distribution. The uncertainty is estimated from the $\chi^{2} / N D F$ of the data normalisation scale factor fit.

Signal Region - The uncertainty from the signal region definition. This is estimated by varying the signal region from 1 to 1.5 and 1.8 sigma and calculating the difference in the number of events in the predicted background. The effect was found to be less than $1 \%$.

The total background systematic uncertainty for the Loose SVT tagger was calculated to be $3 \%$, dominated by the normalisation error [71].

\subsubsection{Results}

The expected and observed limits are outlined in Table 5.10 and are shown in Fig. 5.14 along with the previous p13 [23] result and the region excluded by LEP [21]. When it was published, this result set the world best limits on $\tan \beta$ in the MSSM scenario. 


\begin{tabular}{|c|c|c|}
\hline \multirow{2}{*}{ Signal Mass $(\mathrm{GeV})$} & \multicolumn{2}{|c|}{$\tan \beta$ Limit } \\
\cline { 2 - 3 } & Expected & Observed \\
\hline 90 & 63 & 60 \\
100 & 70 & 71 \\
110 & 71 & 64 \\
120 & 73 & 69 \\
130 & 84 & 75 \\
150 & 93 & 93 \\
\hline
\end{tabular}

Table 5.10: The expected and observed 95\% C.L. $\tan \beta$ limits for the MSSM at tree-level for each $m_{A}$.

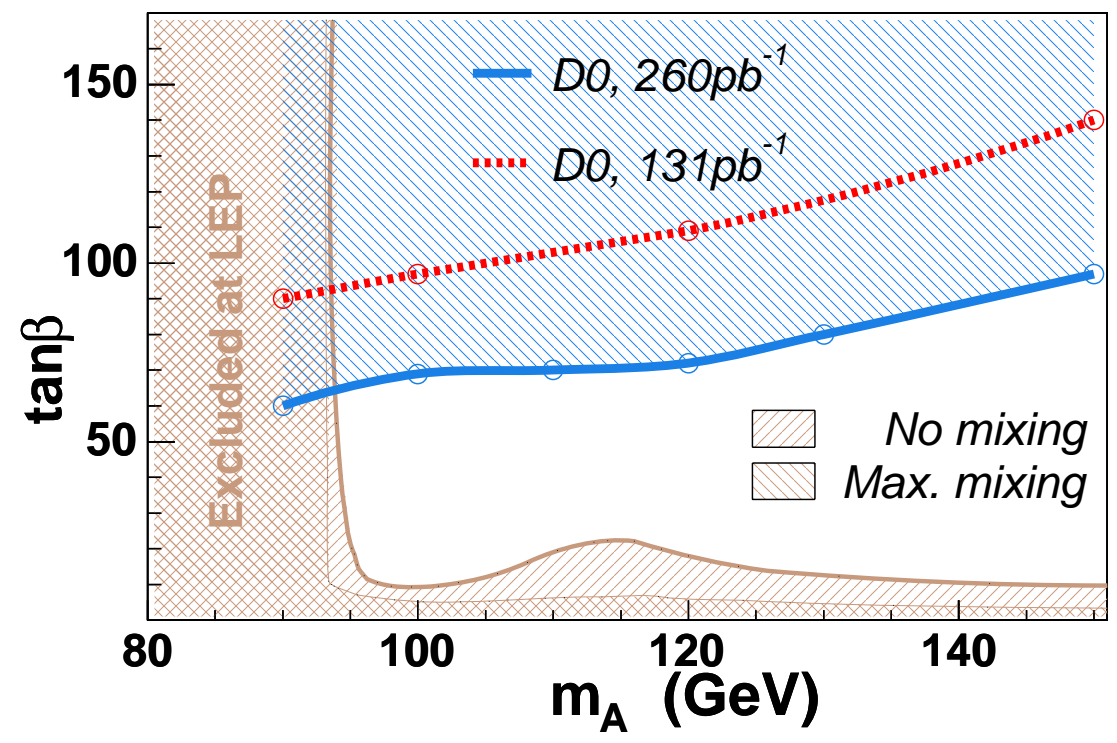

Figure 5.14: The observed 95\% C.L. limits on $\tan \beta$ as a function of $m_{A}$ (area above blue line) and overlayed exclusion limits from the p13 analysis [23] (area above red dotted line), assuming $\tan ^{2} \beta$ cross section enhancement (tree-level results for the MSSM). 


\subsection{Pass2 Analysis}

The goal of the Pass2 analysis was to repeat the Pass1 analysis using the NN $b$ tagger. The differences between the two analyses were:

- Cleaner calorimeter data due to removal of noisy cells.

- Higher luminosity due to recovered bad data between Pass1 and Pass2.

- Smaller signal systematic errors due to a better understanding of the $b$-tagging and jet energy scale uncertainties.

- Use of the NN b-tagger which is a much more performant $b$-tagging tool.

- Reduced statistics for the $h b$ and $b b j$ processes due to the loss of some MC samples between the Pass1 and Pass2 analyses.

The analysis is split into several sections. The event selection is outlined in Section 5.6.1, the problems and investigations in Section 5.6.2 - 5.6.4, the new analysis method in Section 5.6.5, the systematic uncertainties in Section 5.6.6, the tagging point optimisation in Section 5.6.7, the results in Section 5.6.8 and finally conclusions are drawn in Section 5.6.9.

\subsubsection{Event Selection}

Events were selected for inclusion in the analysis if they had:

- 3 - 5 taggable jets with $|\eta|<2.5$ and leading jet cuts of $\mathrm{p}_{T}>45,25$ and $15 \mathrm{GeV}$.

- A PV with $|z|<35 \mathrm{~cm}$.

- Fired a $h b$ trigger.

- At least three $b$-tags.

The optimisation of the b-tagging is discussed in Section 5.6.7. 


\subsubsection{Pass2 Analysis Problems}

Using the same analysis method as set out in Section 5.5.2, the triple tagged di-jet invariant mass distributions are shown in Fig. 5.15 for the Loose SVT (the operating point used in the Pass1 analysis), the Tight NN and the Tight SVT taggers (the operating point whose event yield most closely resembles the Pass1 analysis). Several issues are apparent from the triple tagged distributions:

- A discrepancy exists between the MC (dotted) and data (black) derived background predictions. The same discrepancy, at a lower level, can also be seen in the Pass1 MC triple tag comparison (Fig. 5.12 in Section 5.5.2.2).

- A discrepancy exists between the MC background prediction, the data background prediction and the actual data.

- The Tight SVT tagger in the Pass2 analysis has an event yield similar to the Loose SVT tagger in the Pass1 analysis. This was probably due to a change in the SVT tagging code between Pass1 and Pass2. This resulted in the Loose SVT tagger in Pass1 actually being equivalent to the Pass2 Tight SVT tagger.

\subsubsection{Understanding the Problems}

There were two possible explanations for the problems in the Pass2 analysis:

NN Tagger Problem - The NN tagger is trained on MC di-jet events and the certification is carried out on di-jet events. The possibility therefore existed that the NN tagger's performance was worse on multi-jet events, or that the multi-jet events were not modelled correctly in the MC.

Additional Background - An additional, probably heavy flavour, background which was overlooked in the Pass1 analysis had become more prominent due to the more performant $b$-tagging.

\subsubsection{Neural Network Tagger Investigation}

To test the NN tagger's performance three tests were carried out. Firstly, the tagging rate on multi-jet events and di-jet events in MC was compared. Secondly, the NN input variables were examined on di-jet and multi-jet data to check that the variables are similar in both event types. Thirdly, several new NNs were trained, certified and used in the analysis to check for potential problems in the input variable setup of the NN tagger. 

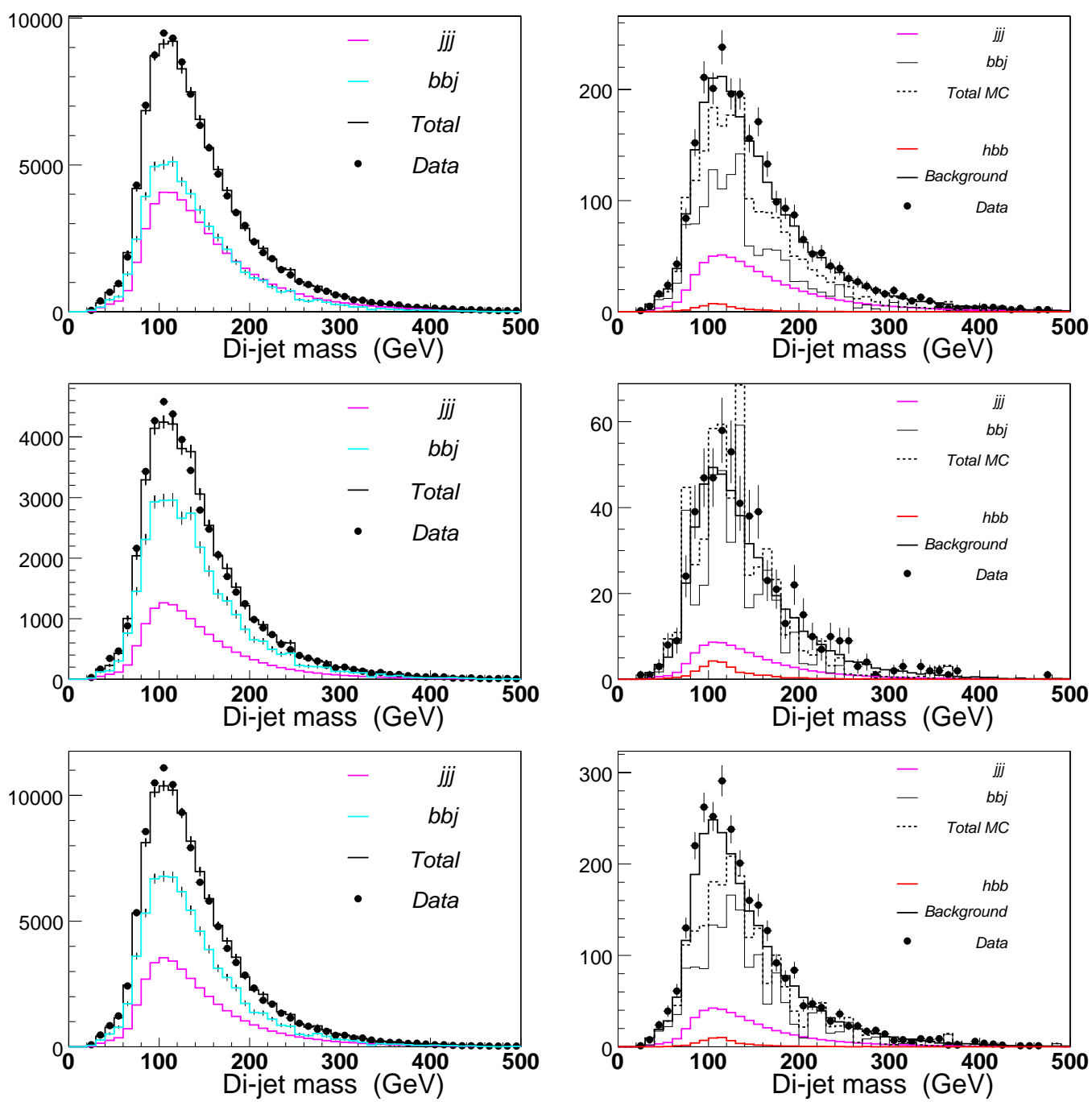

Figure 5.15: The Pass2 double (left) and triple tag (right) di-jet mass distributions for the Loose SVT tagger (top), Tight SVT tagger (middle) and Tight NN tagger (bottom). Data (black points), predicted background (black line) and MC predicted background (dotted black line) are all shown. 


\subsubsection{Multi-Jet Tagging Rates}

The fake rates of the NN tagger in di-jet and multi-jet environments were measured using Pythia [70] QCD MC samples of $u d s g$-jets, and are shown in Fig. 5.16 for a typical NN operating point. The fake rates in the two samples are in agreement within the errors.

Corresponding tests were carried out for $b$ and $c$-jets in the certification of the NN tagger [74]. The tagging rates for multi-jet and di-jet events were again in agreement within errors.

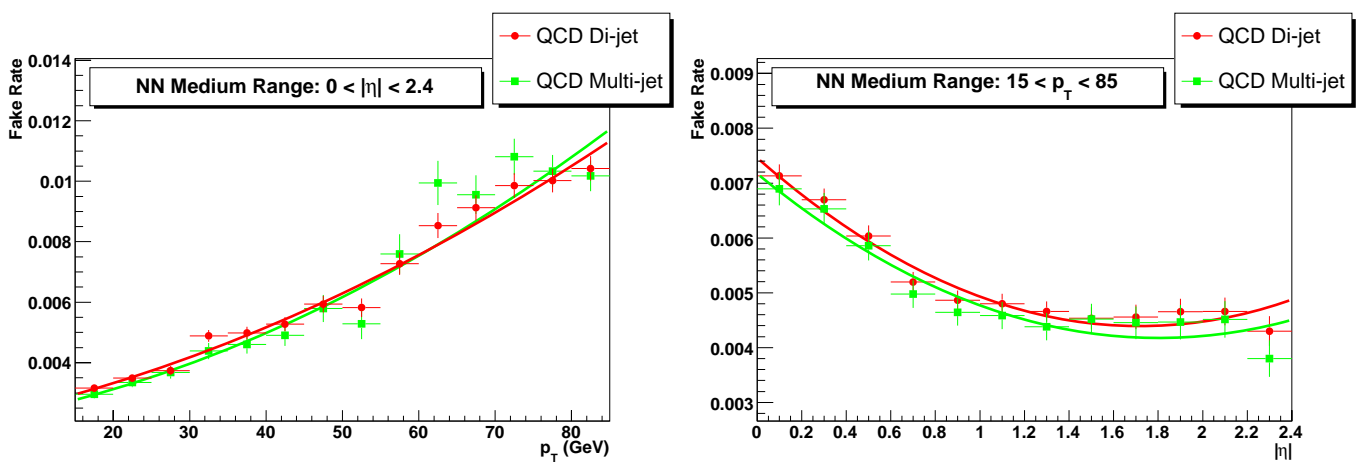

Figure 5.16: The fake-tag rates in di-jet (red circles) and multi-jet events (green squares) for a representative NN operating point (Medium). The fake-tag rates are in agreement within errors.

\subsubsection{Data di-jet and multi-jet Comparisons}

As seen from Fig. 5.16 there is no evidence (in MC) to suggest that there are any appreciable differences between the NN tagging rate in multi-jet and di-jet events. However, differences could exist between data and MC due to differences between multi-jet events in data and MC. For example, correlations could exist between jets in multi-jet events which are incorrectly modelled in $\mathrm{MC}$ or detector issues in multijet events could cause problems with the NN tagging. To test these possibilities comparisons were made between di-jet events, on which the NN tagger was certified, and the multi-jet data events used in this analysis.

In order to compare $b$-tagging variables between multi-jet and di-jet samples there needs to be reasonable confidence that the flavour composition of the samples is similar. To test this assertion the flavour compositions of the jets in a di-jet and multi-jet QCD Pythia MC sample were measured and are shown in Table 5.11. The measurements (which will have large PDF systematic uncertainties) suggest that the expected flavour composition of the two samples are similar enough to allow comparison. 


\begin{tabular}{|c|c|c|c|}
\hline Sample & $b$-Jets (\%) & $c$-Jets (\%) & Fake-Jets (\%) \\
\hline Multi-Jet & $2.7 \pm 0.1$ & $4.8 \pm 0.1$ & $92.5 \pm 0.3$ \\
Di-Jet & $2.7 \pm 0.1$ & $4.6 \pm 0.1$ & $92.7 \pm 0.1$ \\
\hline
\end{tabular}

Table 5.11: The jet flavour composition predicted from Pythia MC samples of di-jet and multi-jet events, errors are statistical only.

The comparison between the input variables for the data di-jet and multi-jet samples is shown in Fig. 5.17 for all jets and in Fig. 5.18 after 1 b-tag was required. There are no significant differences between the input variables in the two samples, although the multi-jet events do have more $b$-like input variables.

\subsubsection{Neural Network Tagger Tests}

The correlations between the input variables, the input jet selection criteria and the input taggers themselves, can be tested by training several new NNs. The following NNs were all designed, trained, certified and tested on the analysis to test various combinations of input taggers, input variables and input jet selections:

NO SVT - The NN is designed to test that the correlations between the SuperLoose SVT tagger and the CSIP and JLIP taggers are correctly modelled in the MC.

Variables: CSIP(2s, 3s, 2w,3w) and JLIP (Prob, Reduced Prob and $N_{\text {Tracks }}$ )

Jet Input Criteria: JLIP Prob $<0.02$ or CSIP COMB $>8$

Tight SVT - This NN was designed to test whether the SuperLoose SVT tagger is modelled correctly in MC.

Variables: Same as the standard NN tagger but all SVT input variables were from the Tight SVT.

Jet Input Criteria: Tight SVT or JLIP Prob $<0.02$ or CSIP COMB $>8$

Loose SVT - Same as above but using the Loose SVT tagger.

Variables: Same as standard NN tagger but all SVT input variables were from the Loose SVT.

Jet Input Criteria: Loose SVT or JLIP Prob $<0.02$ or CSIP COMB $>8$

Tight SVT Jets Only - This NN is designed to test the input jet selection correlations. This NN separates jets with a Tight SVT into signal and background. 

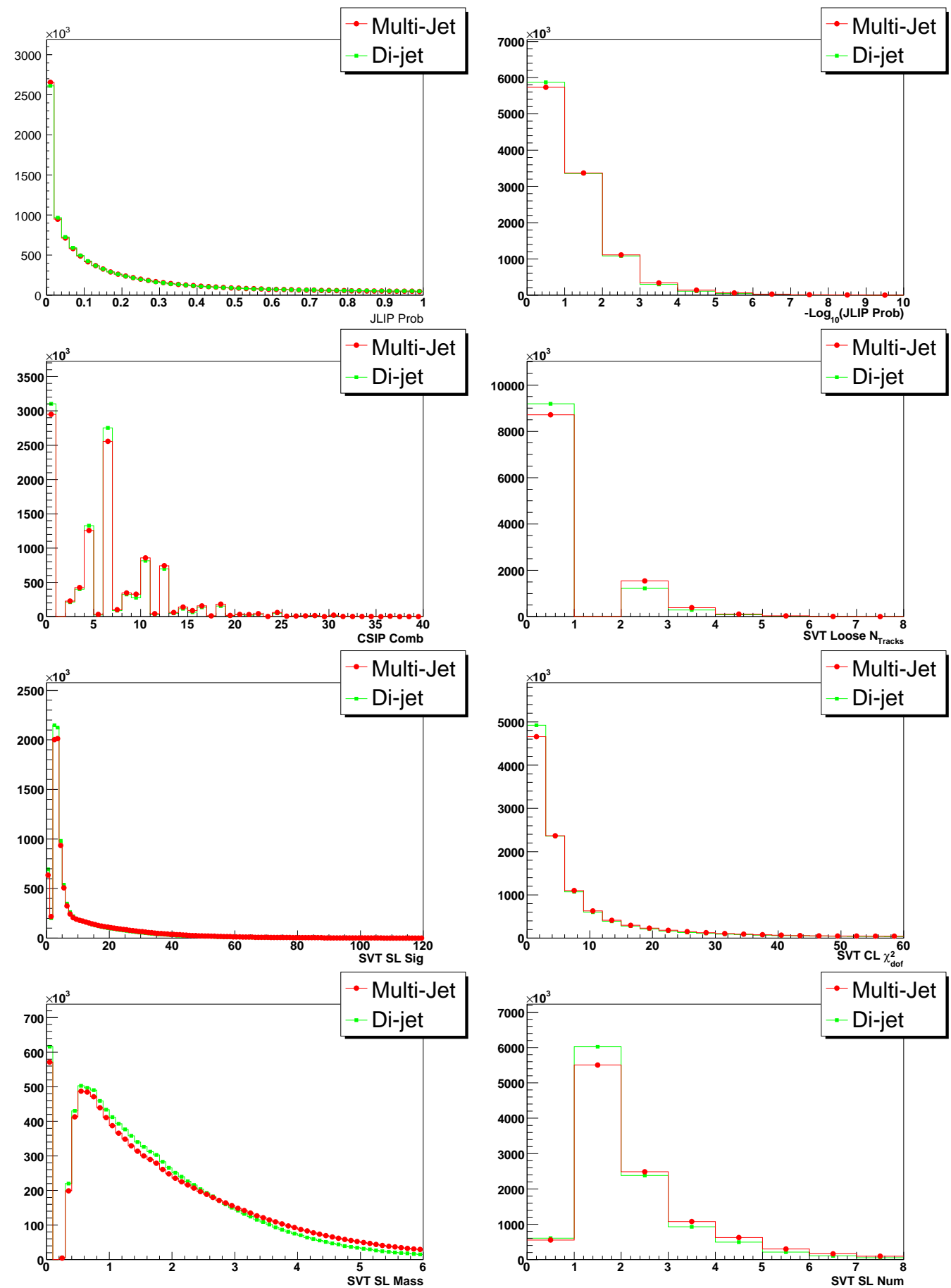

Figure 5.17: NN input variables in the QCD di-jet data sample (green square) and the 3JET multi-jet data sample (red circle) after the NN jet input cuts. The variables are JLIP Prob (top left), $-\log _{10}$ (JLIP Prob)(top right), CSIP Comb (2nd left), $S V T_{L} N_{\text {Tracks }}$ (2nd right), $S V T_{S L}$ DLS (3rd left), $S V T_{S L} \chi_{d o f}^{2}$ (3rd right), $S V T_{S L}$ Mass (bottom left) and $S V T_{S L}$ (bottom right).

The input events are normalised to have equal number. 

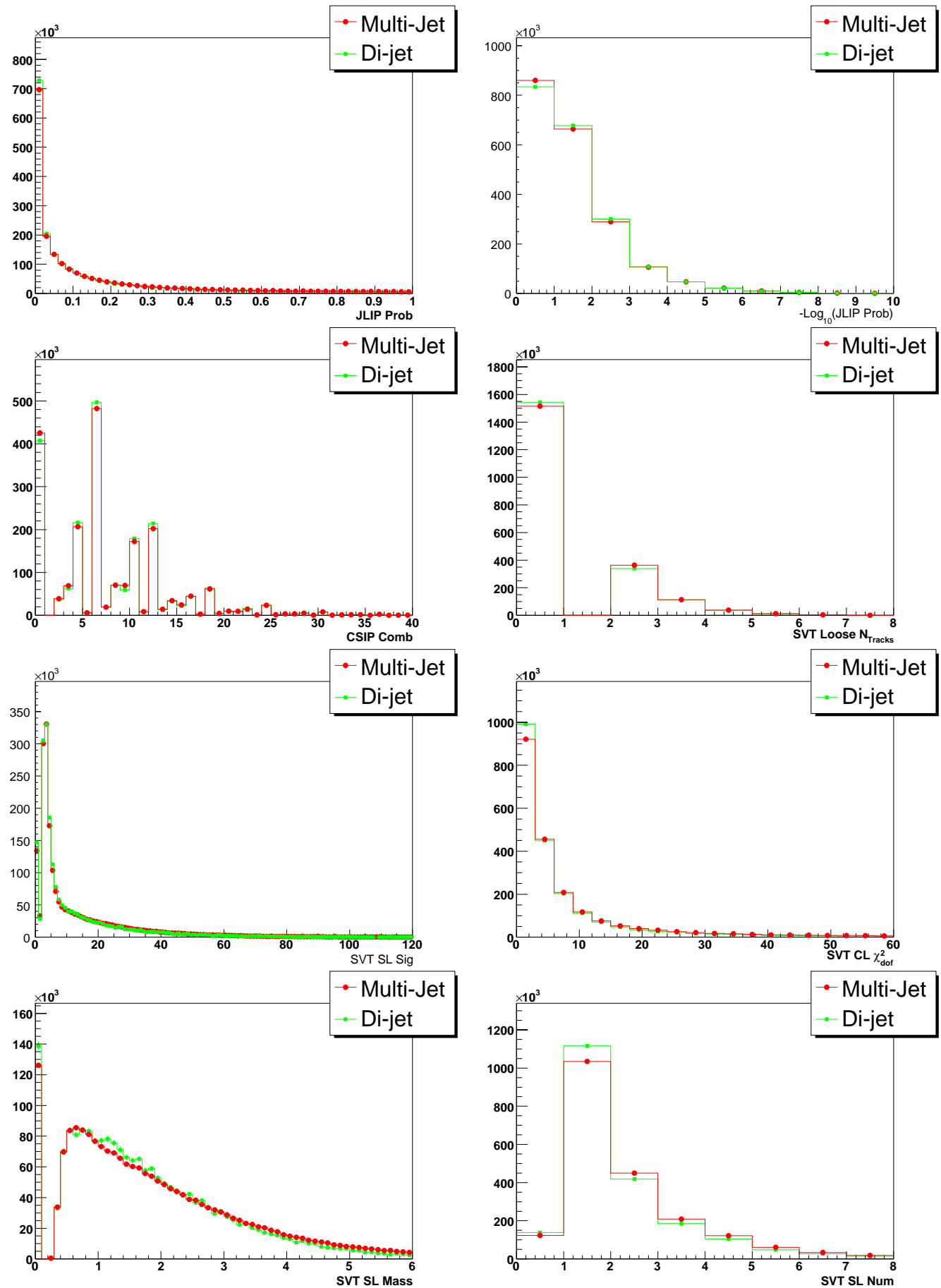

Figure 5.18: NN input variables in the QCD di-jet data sample (green square) and the 3JET multi-jet data sample (red circle) after the NN jet input cuts and 1 tag required in the event. The variables are JLIP Prob (top left), $-\log _{10}$ (JLIP Prob)(top right), CSIP Comb (2nd left), $S V T_{L}$ $N_{\text {Tracks }}$ (2nd right), $S V T_{S L}$ DLS (3rd left), $S V T_{S L} \chi_{d o f}^{2}$ (3rd right), $S V T_{S L}$ Mass (bottom left) and $S V T_{S L}$ (bottom right). The input events are normalised to have equal number. 
Variables: Same as standard NN tagger but all SVT input variables were from the Tight SVT.

Jet Input Criteria: Tight SVT

Loose SVT Jets Only - Same as above but using the Loose SVT tagger.

Variables: Same as standard NN tagger but all SVT input variables were from the Loose SVT.

Jet Input Criteria: Loose SVT

The new NNs all have a worse $b$-tagging performance than the standard NN tagger, although a better $b$-tagging performance than the individual input taggers. When tested in the analysis, the NN taggers which had the worst $b$-tagging performance, gave the best agreement between $\mathrm{MC}$ and data.

\subsubsection{Conclusions}

Checks of the input variables and input composition of the Neural Network all failed to highlight any specific problems. The results also suggested that the worse the performance of the tagger, the better the $\mathrm{MC}$ agreement in the analysis. The MC disagreement in the analysis appears to arise due to the high performance of the NN tagger and not due to a specific problem with the tagging method. This suggests that the disagreement with data arises due to a heavy flavour background which was overlooked in the Pass1 analysis.

\subsubsection{Background Composition Investigation}

The Pass1 analysis assumed that the background and TRFs were both dominated by fake jets. The specially derived FTRF, which was assumed to be unique for the multi-jet data, was used to predict the $j j j$ and $b b j$ backgrounds from data. In the previous section it was shown that the fake-rate in multi-jet events is no different to that in di-jet events. The Pass1 analysis assumption that the FTRF was unique to the multi-jet data sample may have been due to a heavy flavour background which was overlooked. The FTRF therefore contained an additional heavy flavour component, and consequentially disagreed with the di-jet fake rate.

The $b b b b$ background was included in the Pass1 analysis but the cross section was too small for it to be the missing background. However, the $b b b$ process does have a high enough cross section to explain the missing background. The ratio of the $b b b$ process to the $b b j$ process is $1 / 37$ when measured using Alpgen or $1 / 30$ when 
measured using COMPHEP [110]. After a requirement of three $b$-tags this sample would be large enough to make a significant contribution to the background.

This means that the FTRF in Pass1 most probably had heavy flavour contamination. The contamination would affect the $j j j$ prediction causing a heavy flavour component corresponding roughly to the $b b b$ background. However, as the simulated background model was only used as a cross check this would not have affected the limits set by the Pass1 analysis. The data derived background prediction would also have been affected by the heavy flavour contamination. However as the tagging efficiency was so low in the Pass1 analysis ( $~ 30 \%$ b-efficiency) the contamination would have been minimal, and normalising outside the signal region would have corrected any normalisation issues. The shape would have been correctly predicted as the contamination in the FTRF is actually present in the data which is being predicted.

\subsubsection{Monte Carlo Background Composition Model}

Instead of measuring a FTRF for the multi-jet data sample as carried out in Pass1, the fake rate provided by the $b$-ID group [74] was used. The jjj contribution was predicted by using the fake rate to weight the events according to their probability of having $0-5$ tags. Due to the presence of heavy flavour events in the sample (approx $\sim 7 \%$ measured from QCD MC), the predicted $j j j$ contribution will be slightly higher the actual data level.

The $b b j$ and $b b j j \mathrm{MC}$ was used as in Pass1 to model the $b b j$ background, with $\mathrm{j}=u d s c g$, and the $b b j j \mathrm{MC}$ weighted by 0.85 so that the combined MC samples matched the jet multiplicity observed in the data.

No $b b b \mathrm{MC}$ samples were available at the time of the analysis, and due to experimental, computing and time constraints none could be produced for this version of the analysis. Due to the necessity of including a $b b b \mathrm{MC}$ sample a compromise had to be made. An assumption was made that the $b b j \mathrm{MC}$ and the $b b b \mathrm{MC}$ would have a similar spectrum, and the highest $\mathrm{p}_{T}$ jet, which was not a $b$-jet, was treated as the third $b$-jet.

The multi-jet MC samples were normalised to the double tagged data sample. It was assumed that the double tagged data sample consisted of three background components, the double tag fakes (estimated from data), the $b b j \mathrm{MC}$ and a small contribution from $b b b$. The $b b b \mathrm{MC}$ normalisation was fixed to $1 / 33.5$ of the $b b j$ level (from the average of the Alpgen and COMPHEP cross section ratios). The $b b j$ and $b b b \mathrm{MC}$ samples were normalised to the integral of the double $b$-tagged data after substraction of the $j j j$ and the other small MC samples. The agreement of the new 
MC background composition model with the new data background prediction will be compared in the next section.

\subsubsection{Pass2 Analysis Method}

The presence of a significant amount of $b b b$ background invalidates the background prediction method used in Pass1. A new method therefore had to be developed for use with the NN b-tagger.

$\mathrm{TRF}_{2 t a g}$ is the TRF of the jets in an event after at least 2 tags have already been required, with two of the tagged jets randomly excluded. Figure 5.19 shows TRF all and $\mathrm{TRF}_{2 t a g}$, they are different in shape and normalisation in each of the $\eta$ regions. Therefore using $\mathrm{TRF}_{\text {all }}$ and then normalising outside the signal region, as was done in Pass1, would be incorrect as each of the $\eta$ regions needs a different normalisation applied to it.
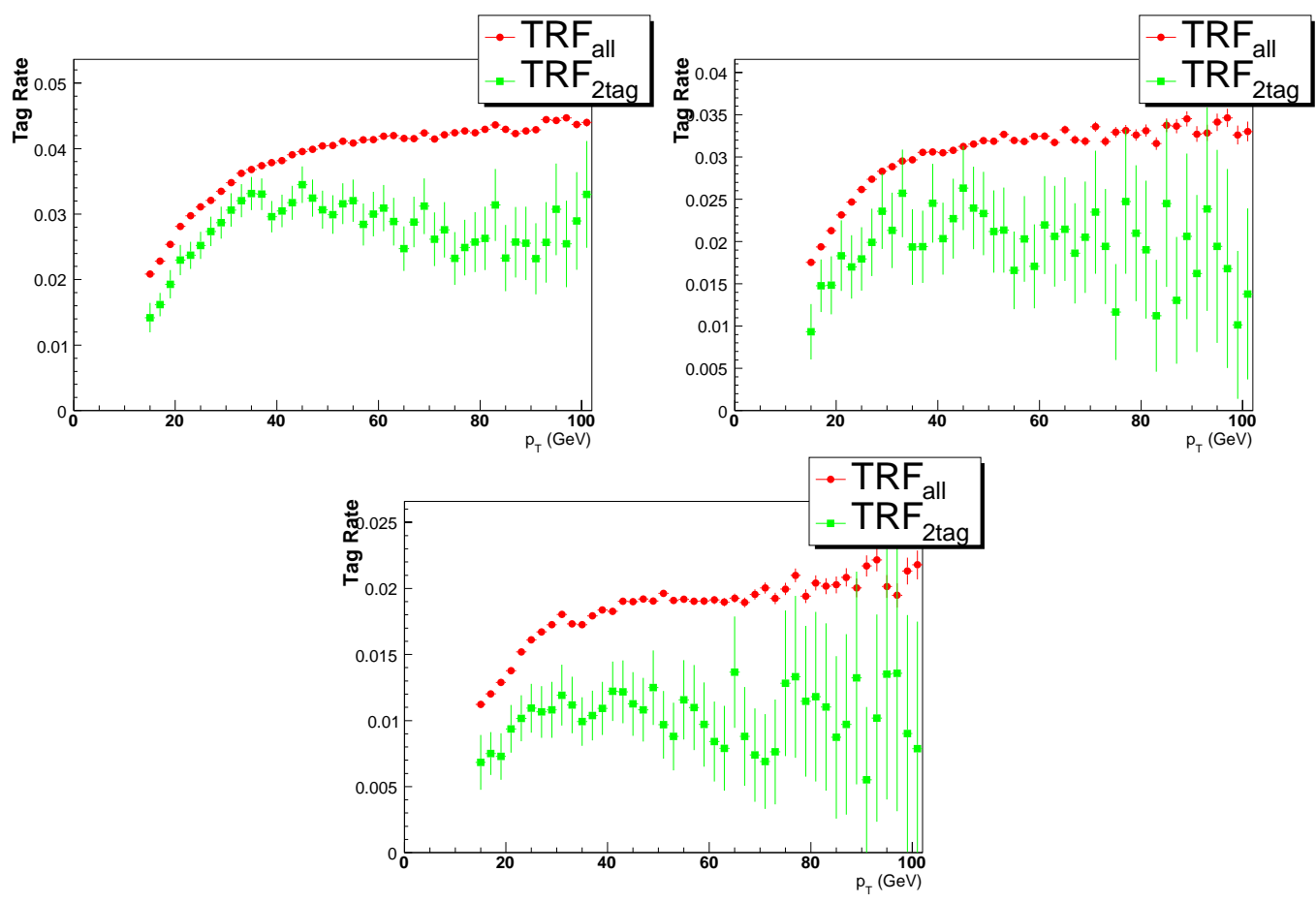

Figure 5.19: The TRFs for all jets in the sample, $\mathrm{TRF}_{\text {all }}$ (red dots), and for jets after 2 tags have been applied, $\mathrm{TRF}_{2 t a g}$ (green squares), in the eta regions $\eta<1.1$ (top), $1.1<\eta<1.5$ (middle) and $1.5<\eta<2.5$ (bottom).

The new analysis method uses $\mathrm{TRF}_{2 t a g}$ to weight the events based upon the probability that one or more of the jets, ignoring the two leading $b$-tagged jets, will be tagged. This provides the shape of the distribution. The predicted distribution is then normalised outside the signal region to account for any signal present in $\mathrm{TRF}_{2 t a g}$. 
The di-jet mass distribution for the MC predicted background, the predicted triple tagged background from data and the triple tagged data are shown in Fig. 5.20 for three of the NN tagger operating points. The predicted background now agrees within errors with the MC background prediction. The background is found to contain a significant proportion of $b b b, b b c$ and $b b j$ events.
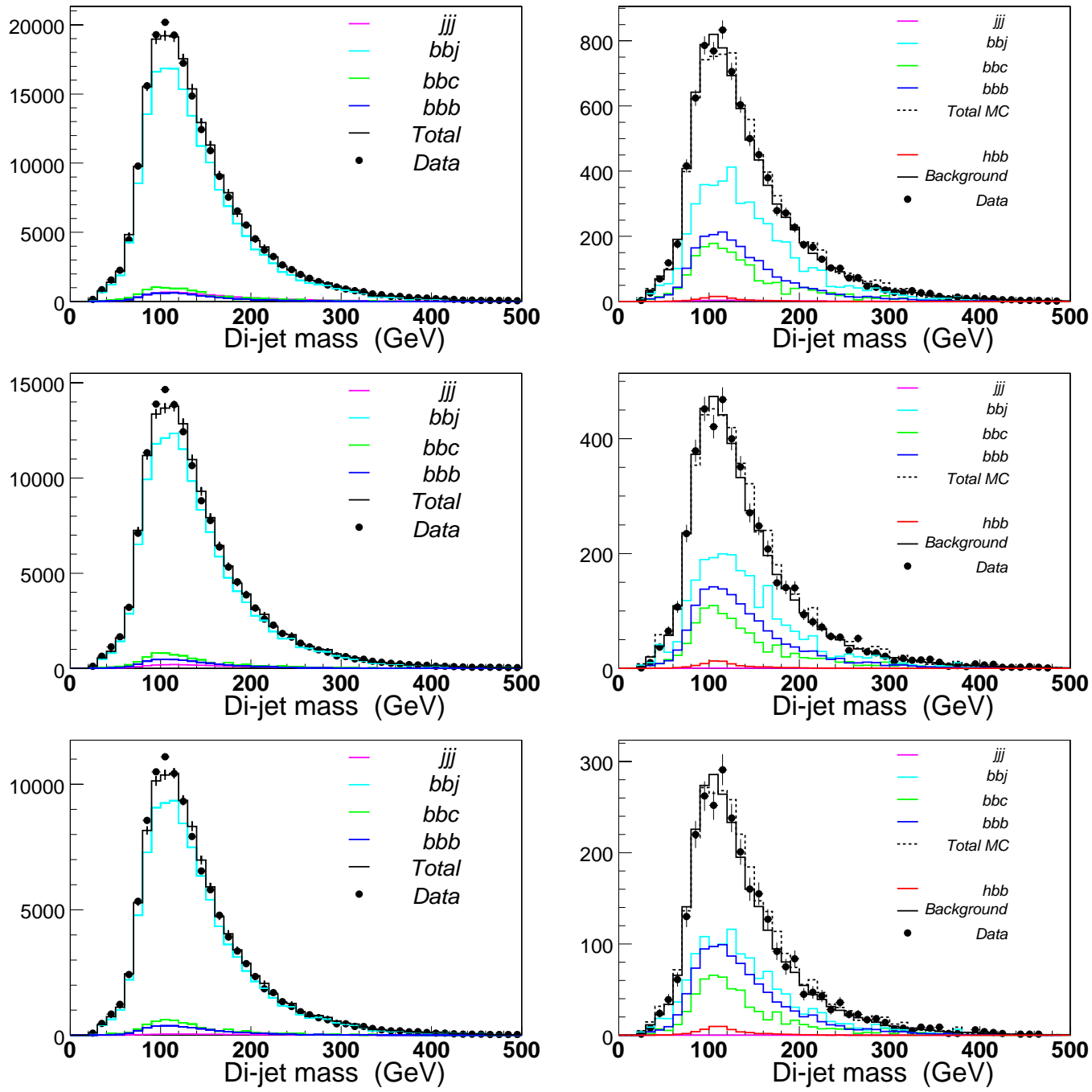

Figure 5.20: The Pass2 double (left) and triple tag (right) di-jet mass distributions for the Loose (top), Medium (middle) and Tight (bottom) NN taggers. Data (black points), predicted background (black line) and MC predicted background (dotted black line) are all shown. A good agreement exists between the data and MC predicted backgrounds for all operating points.

\subsubsection{Systematic Uncertainties}

\subsubsection{Signal Systematic Uncertainties}

The systematic errors are determined in the same manner as in the Pass1 analysis (see Section 5.5.4.1), although with an improved understanding of the $b$-ID [74, 77], 
JES [111] and the jet-ID uncertainties [112].

The signal errors are outlined in Tables 5.12 and 5.13 for the Medium NN and Tight SVT taggers respectively. As expected the errors are smaller than the Pass1 value of $\sim 20 \%$ due to the better JES and $b$-tagging used in the Pass 2 analysis. The systematic errors as a function of the tagging operating point for the Higgs mass point, $m_{H}=120 \mathrm{GeV}$, are shown in Table 5.14 for the NN and SVT taggers. The signal systematic uncertainty decreases slightly as the operating point becomes looser due to the decreased $b$-tagging uncertainty of the looser operating points.

\begin{tabular}{|c|c|c|c|c|c|c|c|c|}
\hline$m_{h}$ & Theory & Lum & Trig & $b$-ID & JES & JER & Jet-ID & Total \\
\hline 90 & 5.0 & 6.5 & 9.0 & 7.3 & 4.0 & 0.7 & 3.5 & 15.2 \\
110 & 5.0 & 6.5 & 9.0 & 7.7 & 3.4 & 0.4 & 2.4 & 15.0 \\
120 & 5.0 & 6.5 & 9.0 & 7.4 & 3.1 & 1.7 & 3.3 & 15.0 \\
130 & 5.0 & 6.5 & 9.0 & 7.1 & 2.2 & 0.3 & 3.7 & 14.8 \\
150 & 5.0 & 6.5 & 9.0 & 7.9 & 1.8 & 0.6 & 2.8 & 14.9 \\
\hline
\end{tabular}

Table 5.12: The systematic errors on the signal in the Pass2 analysis for the Medium NN operating point. All the errors are in $\%$ and are added in quadrature to give the total error. A description of the errors can be found in Section 5.5.4.1.

\begin{tabular}{|c|c|c|c|c|c|c|c|c|}
\hline$m_{h}$ & Theory & Lum & Trig & $b$-ID & JES & JER & Jet-ID & Total \\
\hline 90 & 5.0 & 6.5 & 9.0 & 6.8 & 4.8 & 0.5 & 2.8 & 15.1 \\
110 & 5.0 & 6.5 & 9.0 & 6.3 & 4.9 & 1.0 & 3.6 & 15.0 \\
120 & 5.0 & 6.5 & 9.0 & 6.6 & 4.5 & 1.1 & 2.3 & 14.8 \\
130 & 5.0 & 6.5 & 9.0 & 6.8 & 4.4 & 0.6 & 3.1 & 15.0 \\
150 & 5.0 & 6.5 & 9.0 & 6.5 & 3.4 & 1.5 & 2.7 & 14.6 \\
\hline
\end{tabular}

Table 5.13: The systematic errors on the signal in the Pass2 analysis for the Tight SVT operating point. All the errors are in $\%$ and are added in quadrature to give the total error. A description of the errors can be found in Section 5.5.4.1.

\begin{tabular}{|c|c|c|c|c|c|c|c|}
\hline Error & L2 & Loose & Medium & Tight & V Tight & U Tight & M Tight \\
\hline NN & 13.9 & 14.5 & 15.0 & 15.3 & 15.6 & 15.8 & 16.3 \\
SVT & n/a & 14.5 & 14.8 & 14.8 & n/a & n/a & n/a \\
\hline
\end{tabular}

Table 5.14: The systematic errors, in $\%$, on the signal $\left(M_{h}=120 \mathrm{GeV}\right)$ for the NN and SVT taggers for each of their respective operating points. Only three operating points exist for the SVT tagger. 


\subsubsection{Background Systematic Uncertainties}

The background uncertainties were evaluated in the same manner as in the Pass1 analysis. The systematic errors for each of the NN and SVT operating points are shown in Table 5.15 .

The dominant error is the error on the normalisation which is dependent on the statistics in the triple tag sample. As the operating point becomes looser, and the statistics increase, the error on the background decreases. The errors on the Pass2 NN and SVT are consistent when comparing operating points with similar statistics (e.g. Loose SVT and Tight NN). However, the errors between the Pass1 and Pass2 analyses are not consistent. The Pass1 error for the Loose SVT tagger, which has a distribution and statistics consistent with the Pass2 Tight SVT tagger, has an error of $3 \%$, which is much smaller than the $5.4 \%$ systematic error calculated in the Pass2 analysis.

\begin{tabular}{|c|c|c|c|c|c|c|c|}
\hline Error & L2 & Loose & Medium & Tight & V Tight & U Tight & M Tight \\
\hline NN & 1.0 & 1.5 & 2.0 & 2.6 & 3.0 & 3.9 & 5.2 \\
\hline SVT & n/a & 2.6 & 3.3 & 5.4 & n/a & n/a & n/a \\
\hline
\end{tabular}

Table 5.15: The systematic errors, in \%, on the background for the NN and SVT taggers for each of their respective operating points. Only three operating points exist for the SVT tagger.

\subsubsection{Tagging Optimisation}

The choice of operating point was determined by the sensitivity. Two versions of the sensitivity were considered:

Statistical Sensitivity - The signal, S, divided by the statistical error on the background, B, plus signal:

$$
\frac{S}{\sqrt{S+B}}
$$

Pass2 Sensitivity - The signal divided by the statistical and systematic error on the signal, sigma $a_{S}$, and background, sigma $a_{B}$, added in quadrature:

$$
\frac{S}{\sqrt{S+B} \oplus \sigma_{B} B \oplus \sigma_{S} S} .
$$

The sensitivity as a function of the tagging operating points for the NN and SVT taggers is shown in Fig. 5.21 for the two cases described above. In both cases the NN tagger has a better sensitivity. The optimal operating point is the Medium NN 

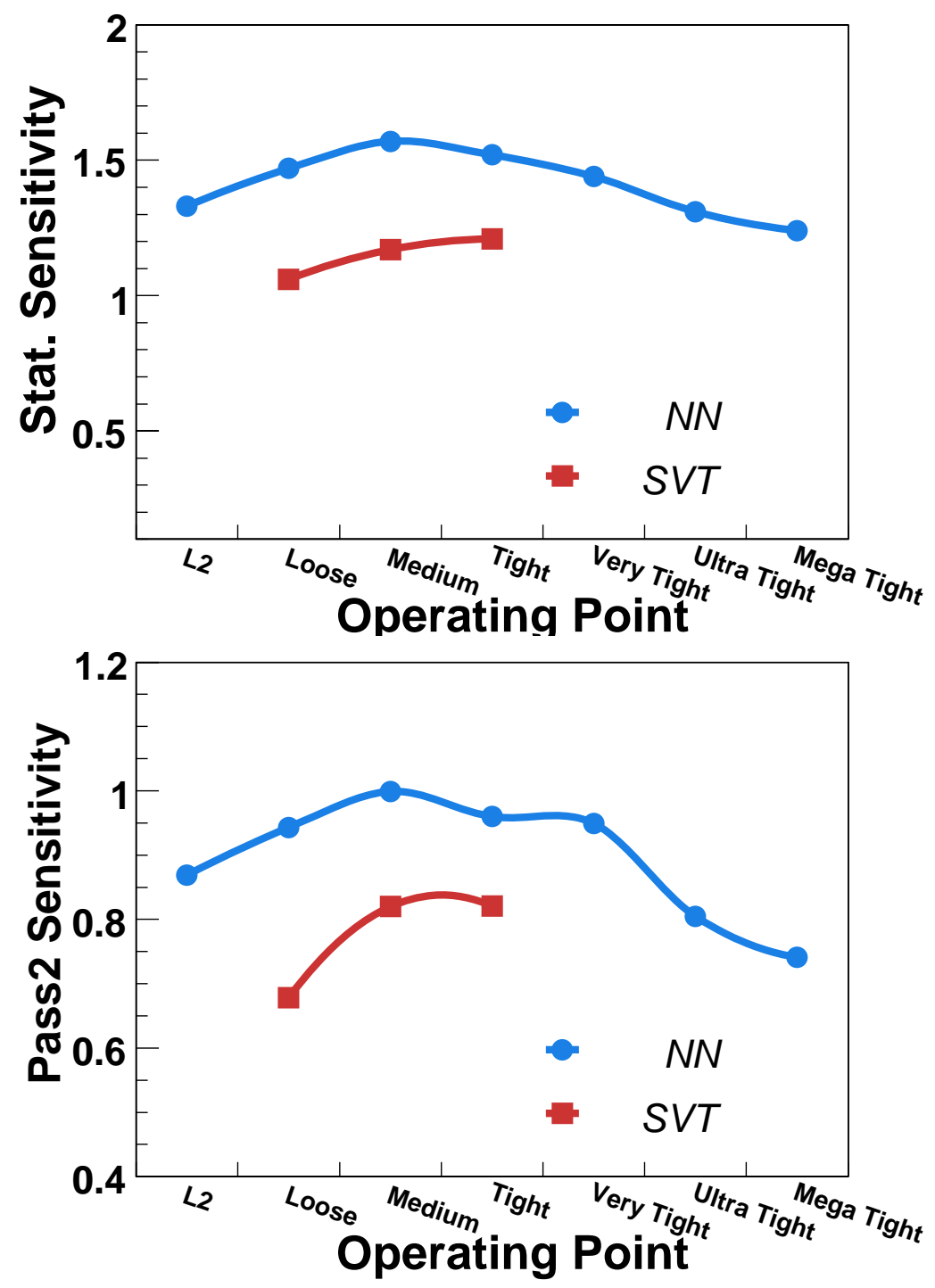

Figure 5.21: Sensitivity as a function of operating point for the NN (blue dots) and SVT (red squares) taggers for $\tan (\beta)=50$. For the cases of: no systematic error on the background prediction (top) and full systematic errors (bottom). 


\subsubsection{Results}

The number of signal, predicted background and data events are shown in Table 5.16.

\begin{tabular}{|c|c|c|c|}
\hline Tagger & Data & Predicted Background & Signal $(\tan (\beta)=50)$ \\
\hline Medium NN & $4730 \pm 69$ & $4685 \pm 116$ & $109 \pm 19$ \\
Tight SVT & $571 \pm 24$ & $566 \pm 35$ & $31 \pm 7$ \\
\hline
\end{tabular}

Table 5.16: The number of events in the signal, predicted background and data triple tagged distribution for the Medium NN and Tight SVT taggers. The errors on the data are statistical only, the errors on the predicted background and signal contain the statistical and systematic errors.

The limits were set using the $\mathrm{CL}_{s}$ method (see Section 5.5.3). The expected limits for the two scenarios of no systematic errors and full systematic errors are shown in Tables 5.17 and 5.18 respectively and also in Fig. 5.22. In both cases the NN improves the limits by $\sim 10 \%$ compared to the SVT tagger. This effectively almost doubles the luminosity of the analysis.

A comparison of the NN tagger to the Pass1 result is also shown in Table 5.18. The Pass1 analysis has better expected limits. This is due to the background errors in the Pass1 analysis being smaller than the background errors used in the Pass2 analysis. It is unclear why the errors in the Pass1 analysis were smaller as a similar method was used to calculate the errors. As a cross check on the assertion that the Pass1 SVT errors were calculated for the wrong operating point, the Pass2 Tight SVT limits were calculated using the Pass1 errors and are shown in Table 5.19. The limits found for the Pass2 SVT and Pass1 SVT are found to be similar when using the same errors.

\begin{tabular}{|c|c|c|}
\hline \multirow{2}{*}{$m_{H}(\mathrm{GeV})$} & \multicolumn{2}{|c|}{$\tan \beta$ Limit } \\
\cline { 2 - 3 } & Pass2 NN & Pass2 SVT \\
\hline 90 & 55 & 62 \\
110 & 63 & 68 \\
120 & 67 & 76 \\
130 & 73 & 77 \\
150 & 86 & 94 \\
\hline
\end{tabular}

Table 5.17: The expected 95\% C.L. $\tan \beta$ limits for the NN tagger and the Pass2 SVT tagger in the MSSM scheme at tree-level. No systematic errors were included in the limit setting. 


\begin{tabular}{|c|c|c|c|c|}
\hline \multirow{2}{*}{$m_{H}(\mathrm{GeV})$} & \multicolumn{4}{|c|}{$\tan \beta$ Limit } \\
\cline { 2 - 5 } & Pass1 SVT & Pass2 NN & Pass2 SVT & Pass2 SVT x2 Lum \\
\hline 90 & 63 & 62 & 72 & 61 \\
110 & 71 & 70 & 76 & 72 \\
120 & 73 & 81 & 84 & 77 \\
130 & 84 & 85 & 92 & 80 \\
150 & 93 & 97 & 107 & 92 \\
\hline
\end{tabular}

Table 5.18: The expected 95\% C.L. $\tan \beta$ limits for the Pass1 SVT tagger, Pass2 NN tagger and the Pass2 SVT tagger in the MSSM scheme at tree-level. Systematic errors were used in the limit setting.

\begin{tabular}{|c|c|c|}
\hline \multirow{2}{*}{$m_{H}(\mathrm{GeV})$} & \multicolumn{2}{|c|}{$\tan \beta$ Limit } \\
\cline { 2 - 3 } & Pass1 SVT & Pass2 SVT \\
\hline 90 & 63 & 67 \\
110 & 71 & 71 \\
120 & 73 & 78 \\
130 & 84 & 82 \\
150 & 93 & 98 \\
\hline
\end{tabular}

Table 5.19: The expected 95\% C.L. $\tan \beta$ limits for the Pass1 SVT tagger and the Pass 2 SVT tagger in the MSSM scheme at tree-level for each $m_{A}$. Limits were calculated assuming the Pass1 systematic error values. 

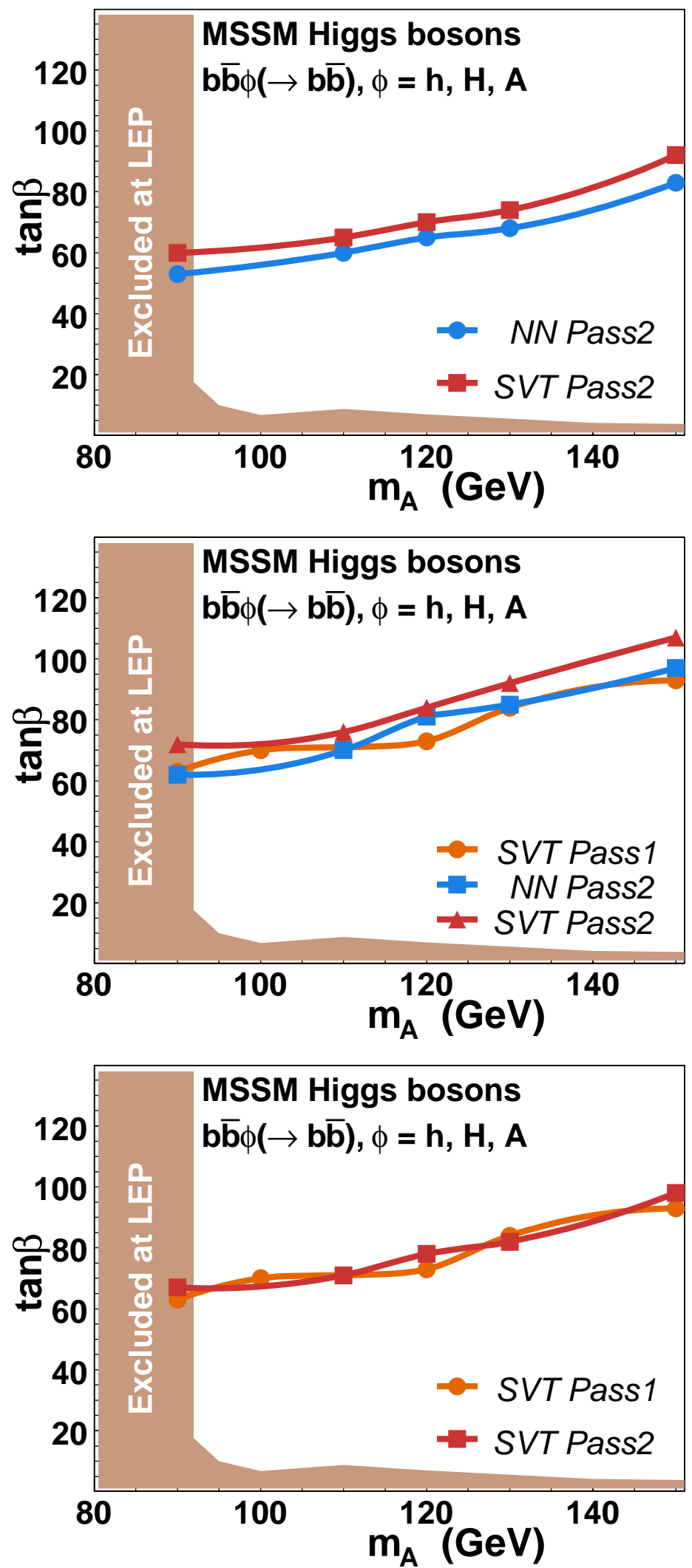

Figure 5.22: Expected limits for the NN and SVT taggers: no systematic errors on the background prediction (top), the Pass 2 systematic errors on the background prediction (middle) and the Pass1 systematic errors for the Pass1 and Pass2 SVT taggers (bottom). 


\subsubsection{Conclusions}

The Pass1 $h b$ analysis was repeated using the NN tagger. The improved $b$-tagging significantly enhanced a heavy flavour background which had been overlooked in the Pass1 analysis. Thus both a new background composition model and analysis technique had to be developed.

The NN tagger significantly improved the sensitivity of the analysis and had the effect of almost doubling the luminosity compared to the SVT tagger used in equivalent circumstances. Direct comparisons between the Pass1 and Pass2 analysis were not possible due to inconsistencies in the background systematic errors.

\section{$5.7 \quad$ p17 Analysis}

The p17 analysis closely follows the methods and background model used in the Pass2 analysis (see Section 5.6), with some improvements.

The analysis is split into several sections. The event selection is outlined in Section 5.7.1, the analysis method in Section 5.7.2, the systematic uncertainties in Section 5.7.3 and finally the results are presented in Section 5.7.4.

\subsubsection{Event Selection}

Events were selected for inclusion in the analysis if they had:

- 3 - 5 taggable jets with $|\eta|<2.5$ and leading jet cuts of $\mathrm{p}_{T}>45,25$ and $15 \mathrm{GeV}$.

- A PV with $|z|<35 \mathrm{~cm}$.

- Fired a $h b$ trigger.

- At least three $b$-tags.

The Tight NN tagger operating point was used to identify $b$-jets.

\subsubsection{Analysis Method}

The background prediction model is the same model as used in the Pass2 analysis.

The MC background cross checks were also similar to those used in the Pass2 analysis, with the inclusion of an additional background, bjjj(j). The contribution from the bjjj(j) background was estimated from data using the $b$-ID $b$-tagging efficiencies; its contribution is found to be small in the double tagged channel and 

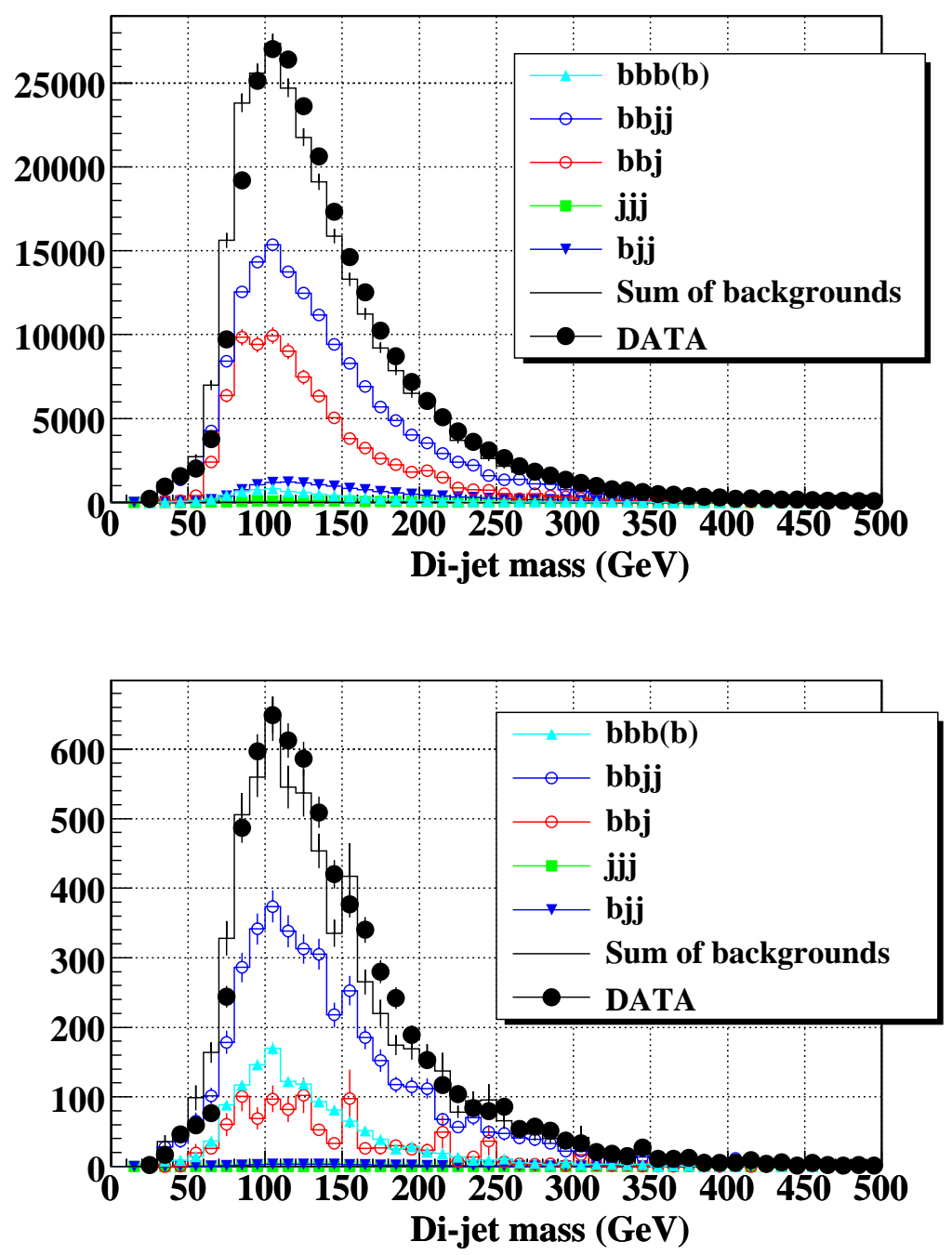

Figure 5.23: Data MC comparison of the di-jet invariant mass distributions in the double $b$-tag sample (top) and the triple $b$-tag sample (bottom).

negligible in the triple tag channel. The $b b j \mathrm{MC}$ was again normalised to the number of events in the double tagged data sample after subtraction of the $j j j$ and $b j j$ contributions. The double tag and triple tag MC cross checks are shown in Fig. 5.23.

Additional attention was paid to possible heavy flavour contributions from $c$ quarks. The $c$-jet efficiency for this operating point is five times lower than the $b$-jet efficiency and processes such as $\operatorname{cjj}(\mathrm{j}), \operatorname{ccj}(\mathrm{j})$ and $\operatorname{ccc}(\mathrm{c})$ were found to be negligible in both the two and three $b$-tag samples. 


\subsubsection{Systematic Uncertainties}

\subsubsection{Signal Systematic Uncertainties}

The sources of signal systematic uncertainties are the same as those used in the p14 analyses (see Section 5.5.4.1). The p17 systematic errors are shown in Table 5.20 [26]. There are a number of differences between the p17 and p14 systematic errors:

- In the p17 analysis the uncertainties due to the limited order in perturbation theory and the PDF set used were also taken account of in the theoretical error. The systematic uncertainty due to the limited order of perturbation theory was estimated by considering the alteration in cross section caused by varying the renormalisation and factorisation scales $(\mu)$ between $\mu / 2$ and $2 \mu$ (these values were motivated from [95, 97]). This resulted in a systematic error which varied from $2-4 \%$ from the lowest to highest mass point. The systematic error associated with the PDF set was evaluated by comparing the cross sections produced when using the 40 different PDF parameterisations provided by the CTEQ collaboration to evaluate errors. The 40 PDF parameterisations correspond to the positive and negative variation of the 20 sources of uncertainties of the PDF. The difference in cross section caused by each of the parameterisations were added in quadrature to give any overall error due to the PDF. This error was estimated to be $11.7 \%$. The p17 theoretical errors were more than twice the size of the p14 errors.

- The trigger and Jet-ID errors were reduced due to greater statistics.

\begin{tabular}{|c|c|c|c|c|c|c|c|c|}
\hline$m_{h}(\mathrm{GeV})$ & Theory & Lum & Trig & $b$-ID & JES & JER & Jet-ID & Total \\
\hline 100 & 12.3 & 6.5 & 4.0 & 8.1 & 4.8 & 0.6 & 0.3 & 17.2 \\
110 & 12.0 & 6.5 & 4.9 & 8.2 & 4.6 & 0.2 & 0.5 & 17.3 \\
120 & 12.1 & 6.5 & 3.6 & 8.3 & 3.9 & 0.1 & 0.4 & 17.0 \\
150 & 13.0 & 6.5 & 4.2 & 8.8 & 2.8 & 0.3 & 0.4 & 17.7 \\
170 & 13.5 & 6.5 & 2.5 & 9.3 & 2.8 & 0.5 & 0.4 & 18.0 \\
\hline
\end{tabular}

Table 5.20: The systematic errors on the signal in the p17 analysis for the Tight NN operating point. All the errors are in $\%$ and are added in quadrature to give the total error. A description of the errors can be found in Section 5.5.4.1. 


\subsubsection{Background Systematic Errors}

The sources of the background systematic errors are the same to those identified in the p14 analyses, although the errors have been evaluated in a different manner. The background systematic errors are outlined in Table 5.21, and the new methods used to calculate the errors are described below.

The uncertainty in the normalisation of the triple tag distribution outside the signal region, is estimated to scale as approximately $\frac{1}{\sqrt{N_{\text {Event }}}}$, where $N_{\text {Events }}$ is the number of events in the predicted background outside the signal region.

The uncertainty in the shape of the predicted triple tag di-jet invariant mass distribution is estimated by comparing the double tag distribution to the triple tag distribution. The difference between the number of events in the two distributions provides the error.

The normalisation errors are consistent between the p17 analysis and the Pass2 analysis. The normalisation error in p17 for the Tight operating point (1.7\%) can be compared to the same error calculated for the Loose operating point in the Pass2 analysis (1.5\%) as they have similar statistics (p17 - 6749, Pass2 - 6798). The Pass2 error is smaller due to knowledge of the shape also being used when calculating the error, if this effect is discounted the error is $1.7 \%$, exactly the same as the p17 error.

\begin{tabular}{|c|c|c|c|}
\hline$m_{h}(\mathrm{GeV})$ & Shape & Normalisation & Total \\
\hline 100 & 1.8 & 1.8 & 2.4 \\
110 & 2.3 & 1.7 & 2.8 \\
120 & 2.4 & 1.7 & 2.8 \\
150 & 1.9 & 1.7 & 2.6 \\
170 & 1.9 & 1.7 & 2.6 \\
\hline
\end{tabular}

Table 5.21: The systematic errors on the background prediction from data in the p17 analysis for the Tight NN operating point. All the errors are in $\%$ and are added in quadrature to give the total error.

\subsubsection{Results}

The limits were set using the $\mathrm{CL}_{s}$ method. Table 5.22 and Fig. 5.24 show the observed and expected $95 \%$ confidence limits on $\tan \beta$ as a function of $m_{A}$. Figure 5.25 shows the triple $b$-tagged di-jet invariant mass distributions for $m_{A}=120 \mathrm{GeV}$ at the observed CL. 


\begin{tabular}{|c|c|c|}
\hline \multirow{2}{*}{ Signal Mass $(\mathrm{GeV})$} & \multicolumn{2}{|c|}{$\tan \beta$ Limit } \\
\cline { 2 - 3 } & Observed & Expected \\
\hline 100 & 46 & 50 \\
110 & 57 & 58 \\
120 & 60 & 62 \\
150 & 85 & 84 \\
170 & 121 & 104 \\
\hline
\end{tabular}

Table 5.22: The observed and expected 95\% C.L. $\tan \beta$ exclusion limits for the p17 analysis in the MSSM scheme at tree-level.

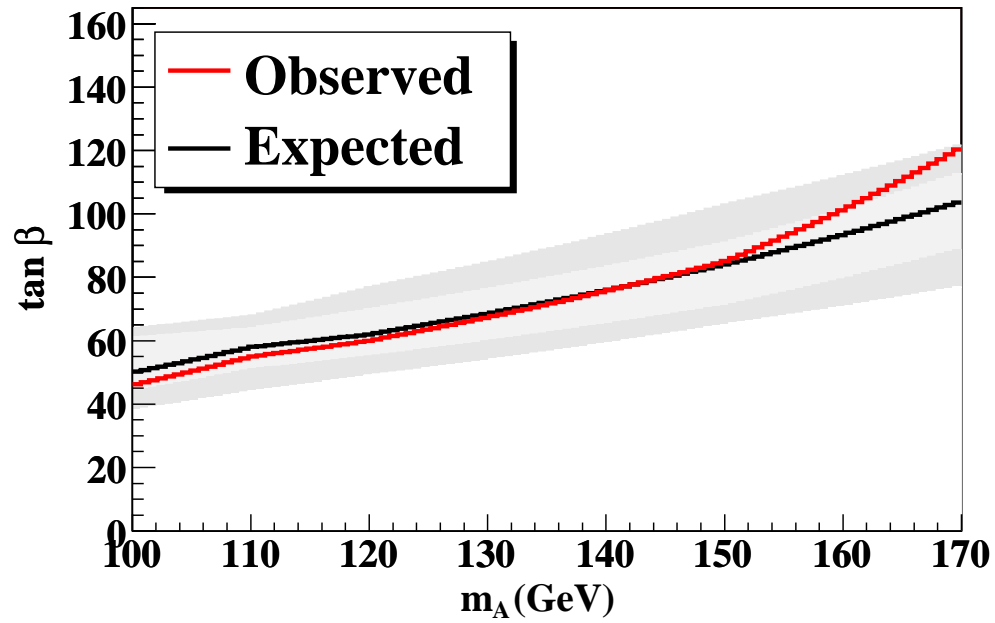

Figure 5.24: The observed and expected $95 \%$ confidence limits on $\tan \beta$ as a function of $m_{A}$, assuming $(\tan \beta)^{2}$ cross section enhancement. The error bands indicate the $\pm 1 \sigma$ and $\pm 2 \sigma$ ranges on the expected limit. 


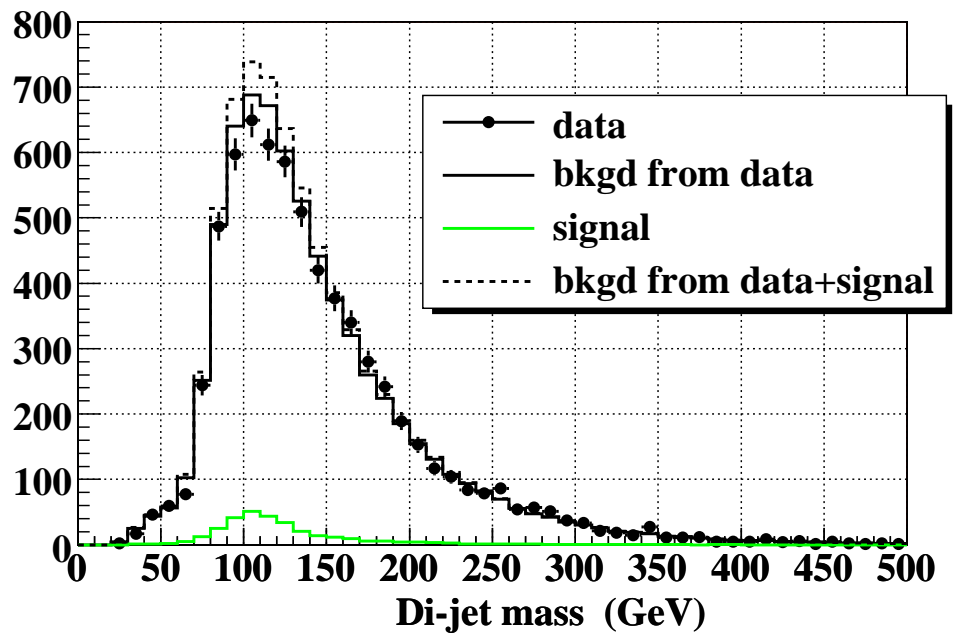

Figure 5.25: The di-jet invariant mass distribution for the data (circles), signal (solid grey line), background (solid black line) and combined signal and background (dashed black line) for $m_{A}=120 \mathrm{GeV}$ at the observed $95 \%$ confidence limit $(\tan \beta=60)$.

\subsection{Conclusion}

Three generations of the $h b$ analysis have been presented in this chapter. The p14 Pass1 analysis has been published and set world best limits on the production of neutral Higgs bosons in the MSSM scheme.

The p14 Pass1 analysis has since been updated with the p14 Pass2 version of the analysis using the NN b-tagging. This required significant effort to implement due to problems revealed by the more efficient $b$-tagging. The new $b$-tagging was shown to effectively double the luminosity of the analysis.

The latest version of the analysis has used the new NN $b$-tagging tool (including the new analysis model and method which were developed), the new $b$-tagging triggers and $0.9 \mathrm{fb}^{-1}$ of data to set improved limits on the production of neutral Higgs bosons in the MSSM, as presented at ICHEP 2006. An improved version of this analysis is being prepared for publication. 


\section{Chapter 6}

\section{Conclusions and Outlook}

\subsection{Summary}

One of the most important and as yet unresolved issues in particle physics is electro weak symmetry breaking and the origin of mass in the Standard Model (SM). The Higgs mechanism has been proposed to explain this issue, and the search for the Higgs boson is currently one of the major goals of particle physics.

Supersymmetry (SUSY) is a popular extension to the SM which solves the 'hierarchy' problem, allows the forces to be unified at high energy scales and allows for the inclusion of gravity. In the Minimal Supersymmetric Extension to the SM (MSSM), the Higgs sector consists of 5 Higgs bosons, 3 of which are neutral and have enhanced couplings to $b$-quarks. In the MSSM this can make bottom quark associative production channels the dominant Higgs production channel, and searches in the $3 b$-jet channel allow strict constraints to be placed on the parameter space of the MSSM.

Both $\mathrm{D} \varnothing$ and the Tevatron are performing well. DØ's data-taking efficiency is above $85 \%$ and the Tevatron has surpassed its instantaneous and weekly integrated luminosity goals. The Tevatron expects to deliver up to $8 \mathrm{fb}^{-1}$ of data by the end of 2009.

This is an exciting time for the Higgs searches at the Tevatron, with the dataset rapidly expanding. There is a wide variety of Higgs searches at D $\varnothing$, both in the SM and SUSY scenarios, with 6 papers published and more awaiting publication. The D $\varnothing$ and CDF SM Higgs searches have now been combined for the first time. The combined limit is a factor of 10(4) away at Higgs mass of $115(160) \mathrm{GeV}$, but with planned improvements and more data these limits are expected to decrease rapidly. At $3 \mathrm{fb}^{-1}$ a Higgs mass of $115 \mathrm{GeV}$ can be excluded at $95 \%$ confidence level, and 
with $8 \mathrm{fb}^{-1}$ there could be $3 \sigma$ or even $5 \sigma$ evidence for a SM Higgs boson with mass less than $130 \mathrm{GeV}$.

The work in this thesis has made a large contribution towards $\mathrm{D} \varnothing$ and its physics programmes. The work on the L3 tracking revealed problems with the L3 SMT clustering, and led to several improvements and fixes being implemented in the unpacking tool. These include: new pedestal thresholds, cluster errors, splitting and merging algorithms and 'hot' SVX chip protection. These improvements increased the L3 tracking purity by $\sim 1-8 \%$, the efficiency by $\sim 5 \%$ and the quality, by increasing the number of SMT hits per track by up to $70 \%$. These improvements have benefited almost every physics group's triggers. The upgrades have improved the performance of all the tools which rely on the tracking, especially the impact parameter (IP) and secondary vertex (SVT) b-tagging tools outlined in this thesis.

New triggers, designed for the multi-jet Higgs analysis, obtained an additional factor of four extra rejection whilst improving the overall efficiency of the triggers by $\sim 40 \%$. The additional rejection was vital in allowing the triggers to effectively function at high instantaneous luminosities. The L3 IP $b$-tagging tool, demonstrated and tested in a trigger for the first time, allowed large gains in rejection to be achieved for very little loss in signal efficiency. The L3 IP $b$-tagging tool was measured to have an efficiency of $\sim 90 \%$ on a 3 -jet sample whilst cutting the background by $\sim 90 \%$. This pioneered the use of $b$-tagging in the trigger, and has since been adopted by other analyses.

Another $b$-tagging tool which locates displaced secondary vertices in jets has been developed and optimised for use in the L3 trigger. The new tool has a large potential for reducing background rates at little expense to the trigger efficiency. Six potential operating points have been identified for use; the points have signal efficiencies varying from $93 \%$ to $54 \%$ for 'rates' ranging from $43 \%$ to $4 \%$. The tool's time consumption was measured as $1.4 \mathrm{~ms}$ at an instantaneous luminosity of $\sim 15 \times 10^{30} \mathrm{~cm}^{-2} \mathrm{~s}^{-1}$.

DØ's first Neural Network $b$-tagging tool was developed and certified ${ }^{1}$. The performance of the NN tagger was measured and parameterised into tag rate functions for six operating points with fake 'rates' ranging from $0.3 \%$ to $4.0 \%$. The NN tagger demonstrated a considerable improvement in performance compared to the standard taggers. For a fixed fake rate, relative improvements in signal efficiency range from $\sim 40 \%$ for the tightest operating to $\sim 15 \%$ for the loosest operating point. Fake rates, for a fixed signal efficiency, are typically reduced to between a quarter and a

\footnotetext{
${ }^{1}$ Measured, tested and approved for use on data after being reviewed by the $\mathrm{D} \emptyset$ collaboration.
} 
third of their value. The improved tagging has significantly increased the sensitivity of the Higgs search at DØ, and is a vital component of the future projections. The benefit from the new tagger is expected to be the equivalent of doubling the luminosity on a double $b$-tag analysis, and benefits a wide range of physics groups, including the single top, top, and Higgs groups.

Three generations of the search for neutral MSSM Higgs bosons have been presented. The p14 Pass1 analysis has been published and set new world best limits on the production of neutral Higgs bosons in the MSSM scheme. The p14 Pass1 analysis has since been updated with the p14 Pass2 version of the analysis using the NN $b$-tagging. This required significant effort to implement due to problems revealed by the more efficient $b$-tagging and resulted in a new analysis method being developed. The new $b$-tagging was shown to effectively double the luminosity of the analysis.

The latest version of the analysis used the new NN $b$-tagging tool (including the new analysis method), the new $b$-tagging triggers and $0.9 \mathrm{fb}^{-1}$ of data to set a new preliminary world best limit on the production of MSSM neutral Higgs bosons. This result was presented at ICHEP 2006, and is being prepared for publication in early 2007.

\subsection{Future Work}

The two L3 $b$-tagging tools demonstrated in this thesis could be combined in simple or sophisticated ways to further increase the effectiveness of the $b$-tagging in the triggers, there is even potential to use a NN to combine the various variables from the two tools. The combination of the $b$-tagging tools could be used in physics triggers to either cope with higher instantaneous luminosities, or even to allow other, less powerful cuts, to be loosened. In the higher instantaneous luminosity of Run IIb, the $b$-tagging tools will prove vital in reducing the rate for the top, single top and Higgs triggers.

The NN b-tagging tool can be improved by a more refined selection of input variables, creating different NNs for low and high energy jets, creating a new NN to separate $b$ and $c$ jets and by improving the MC simulation. Potentially the biggest improvement that could be made to the $b$-tagging would be the development of a system to allow the NN output to be used as a continuous variable, as opposed to the current system where only certain 'certified' operating points can be used. This would allow any NN output value to be used as a b-tagging cut. More interestingly, it would allow the NN output values from each of the jets in an event to be combined 
into a single variable ${ }^{2}$, which could potentially be a more powerful discriminating variable than the individual jet NN output values.

The multi-jet Higgs analysis could be improved in several ways: a NN event selection, a more sophisticated $b$-tagging optimisation, including more than one trigger in the analysis and by splitting the analysis into orthogonal 3 and $4 b$-jet channels to increase the sensitivity.

\footnotetext{
${ }^{2}$ For example, for 2 jets with NN output $P_{1}$ and $P_{2}$, a more powerful discriminating combination may be $\ln \left(P_{1} \times P_{2}\right)$.
} 


\section{References}

[1] F. Halzen, A. Martin, "Quarks \& Leptons", John Wiley \& Sons, 1984, ISBN 0-471-81187-4.

[2] D. Griffiths, "Introduction to Elementary Particles", John Wiley \& Sons 1987, ISBN 0-471-60386-4.

[3] S. Dawson, "SUSY and Such", hep-ph/9612229.

[4] D. I. Kazakov, "Beyond the Standard Model (in Search of Supersymmetry)", hep-ph/0012288 v2.

[5] W. Yao et al. (Particle Data Group), "Supersymmetry", J. Phys. G 33. 1 (2006).

[6] P. Higgs, "Broken Symmetries, Massless Particles and Gauge Fields", Phys. Lett. 12, 132 (1964).

[7] P. Higgs, "Broken Symmetries and the Masses of the Gauge Bosons", Phys. Lett. 13, 508 (1964).

[8] P. Higgs, "Spontaneous Symmetry Breakdown without Massless Bosons", Phys. Rev. 145, 1156 (1966).

[9] Physics of and with Leptons, http://www-zeus.physik.uni-bonn.de/ rbrock/feynman/vtp_ws0506/index.html.

[10] J. Goldstone, A. Salam, S. Weinberg, "Broken Symmetries", Phys. Rev. 127, 965 (1962).

[11] S. Weinberg, "A Model of Leptons", Phys. Rev. Lett. 19, 1264 (1967)

[12] S. Glashow, J. Iliopoulos, L. Maiani, "Weak Interactions with Lepton-Hadron Symmetry", Phys. Rev. D2, 1285-1292 (1970). 
[13] The LEP Working Group for Higgs Boson Searches, "Search for the Standard Model Higgs Boson at LEP", Phys. Rev. Letters B, Volume 565, pp. 61-75 (2003).

[14] The TEVNPH Working Group, "Combined D $\varnothing$ and CDF Upper Limits on Standard-Model Higgs-Boson Production", CDF Note 8384 DØNote 5227.

[15] The CDF and D Collaborations, "Results of the Tevatron Higgs Sensitivity Study", FERMILAB-PUB-03/320-E.

[16] The LEP Electroweak Working Group, "A Combination of Preliminary, Electroweak Measurements and Constraints on the Standard Model", http:// lepewwg . web . cern. ch/LEPEWWG/plots/winter2006/.

[17] K. Riesselmann, "Limitations of a Standard Model Higgs Boson", hep$\mathrm{ph} / 9711456$.

[18] B. Lee, C. Quigg, H. Thacker, "Weak interactions at Very High Energies: The Role of the Higgs-Boson Mass", Phys. Rev. D 16, 15191531 (1977).

[19] J. Gunion, H. Haber, G. Kane, S. Dawson, "The Higgs Hunter's Guide", Addison-Wesley (1990).

[20] B. Ananthanarayan, G. Lazarides, Q. Shafi, "Top-Quark-Mass Prediction from Supersymmetric Grand Unified Theories", Phys. Rev. D44, 16131615 (1991).

[21] The LEP Working Group for Higgs Boson Searches, "Search for Neutral MSSM Higgs Bosons at LEP", Eur. Phys. J. C 47, 547-587 (2006).

[22] The CDF Collaboration, "Search for Neutral Supersymmetric Higgs Bosons in $p \bar{p}$ Collisions at $\sqrt{s}=1.8-\mathrm{TeV} "$, Phys. Rev. Lett. 86 (2001) 4472-4478.

[23] A. Haas, A. Kharchilava,, G. Watts, "D $\varnothing$ Search for Neutral Higgs Bosons at High $\tan \beta$ in Multi-jet Events Using p13 Data", DØNote 4290.

[24] The DØ collaboration, "Search for Neutral Supersymmetric Higgs Bosons in Multijet Events at $\sqrt{s}=1.96$ TeV", Phys. Rev. Lett. 95 (2005) 15801.

[25] A. Goussiou, Y. Pogorelov, P. Svoisky, "Search for Neutral Higgs Bosons at High $\tan \beta$ in the $b(h / H / A) \rightarrow b \tau^{+} \tau^{-}$Channel", DØNote 5246. 
[26] F. Couderc, P. Jonsson, M. Michaut, S. Robinson, T. Scanlon, B. Tuchming, "Search for Neutral Higgs Bosons at High $\tan \beta$ in Multi-Jet Events", DØNote 5203.

[27] Fermi National Accelerator Laboratory (Fermilab) Homepage http://www. fnal.gov/

[28] Fermilab Beams Division, "Collider Run II Peak Luminosity", http:// www-bdnew . fnal .gov/operations/lum/lum.html

[29] V.M. Abazov, et al. (D0 Collaboration), "The Upgraded DØ Detector", Nucl. Instrum. and Methods A 565, 463-537 (2006).

[30] Fermilab Beams Division, "Run II Parameter List", http://www-bd.fnal. gov/runII/parameters.pdf.

[31] The DØ Operations Group, "Daily Data Taking Efficiency", http:// d0server1.fnal.gov/Projects/Operations/DORunII_DataTaking.htm

[32] G. Blazey, "Dø Performance and Planned Improvements", Talk given to the Derparment of Energy High Energy Physics Advisory Panel, September 2005.

[33] R. Lipton, "Layer 0 performance", DØ Workshop, August 2006.

[34] DØ Collaboration, "Beam Tests of the DØ Uranium Liquid Argon End Calorimeter", Nucl. Instr. and Methods A 32453 (1993).

[35] G. Borissov, "Status of DØ Track Reconstruction", Talk given at All DØ Meeting, 14 Feb 2003.

[36] G. Borissov, "Ordering a Chaos or... Technical Details of AA Tracking", Talk given at All DØ Meeting, 28 Feb 2003.

[37] A. Khanov, "HTF: Histogramming Method for Finding Tracks. The Algorithm Description.", DØNote 3778.

[38] P. Hough, "Machine Analysis of Bubble Chamber Pictures", International Conference on High Energy Accelerators and Instrumentation, p554-556 (1959).

[39] H. Greenlee, "The DØ Kalman Track Fit", DØNote 4303.

[40] H. Greenlee, "The DØ Interacting Propagator", DØNote 4293. 
[41] A. Garcia-Bellido, S. Lager, F. Rizatdinova, A. Schwartzman, G. Watts, "Primary Vertex Certification in p14", DØNote 4320.

[42] A. Schwartzman, C. Tully, "Primary Vertex Reconstruction by Means of Adaptive Vertex Fitting", DØNote 4918.

[43] G. Blazey, et. al., "Run II Jet Physics: Proceedings of the Run II QCD and Weak Boson Physics Workshop", hep-ex/0005012.

[44] J. Agram et. al., "Jet Energy Scale at DØ RunII", DØNote 4720.

[45] C. Gerber, E. Chabalina, G. Garzon, "Taggability in Pass2 p14 Data", DØNote 4995.

[46] A. Schwartzman, M. Narain, "Track-Jet Studies Using a 3-Dimensional Simple Cone Jet Algorithm", DØNote 3919.

[47] C. Clement et al., "MuonID Certification for p14", DØNote 4350.

[48] R. Demina, A. Khanov, F. Rizatdinova, "b-Tagging with Counting Signed Impact Parameter Method", DØNote 4049.

[49] D. Bloch, B. Clément, D. Gelé, S. Greder, Isabelle Ripp-Baudot, "Performance of the JLIP b-tagger in p14", DØNote 4348.

[50] A. Schwartzman, M. Narain, "b-quark Jet Identification with Secondary Vertex Reconstruction using DØReco Version p14", DØNote 4414.

[51] K. Hanagaki, J. Kasper, "Identification of b-jet by Soft Muon", DØNote 4867.

[52] R. Illingworth, "Raw Data Format and Unpacking for the SMT", DØNote 3829.

[53] D.Bauer, R.Illingworth, "Level 3 Trigger: Clustering in the SMT", DØNote 3822.

[54] L3 CFT Unpacking Tool, http://www-d0.fnal.gov/d0dist/dist/packages/ $13 f$ cftunpack/p16-br-04/.

[55] D. Whiteson, "Global Track Finding at Level 3", DØNote 3808.

[56] Imperial College DØ Group, "A Level 3 CFT Tracking Tool", DØNote 3779.

[57] N. I. Chernov, G. A. Ososkov, "Effective Algorithms for Cirlce Fitting", Computer Physics Communications 33 (1984) p329-333. 
[58] C. Barnes, P. Jonsson, R. Beuselinck, "Vertexing for the Level 3 Trigger", DØNote 4271.

[59] Level 3 Jet Tool Documentation, http://www-d0.fnal.gov/computing/ algorithms/level3/jets/L3TJet_overview.html.

[60] V. Buscher, "Calorimeter Clustering Tool for the DØ Level 3 Trigger at Run II", http://www-d0.fnal.gov/computing/algorithms/level3/jets/ 13fcalcluster.ps.

[61] E. Busato, B. Andrieu, "Jet Algorithms in the DØRun II Software: Description and User's Guide", DØNote 4457.

[62] Level 3 Impact Parameter Jet B-tag page, http://www-d0.fnal.gov/ computing/algorithms/level3/b-tagging/L3Btag.html.

[63] C. Barnes, R. Jesik, "Level 3 Triggers for Bs Mixing", DØNote 4272.

[64] D. Bauer, V. Lesne, R. Jesik, "Level 3 Muon Tools: p16 certification", DØNote 4470.

[65] The DØRuns Quality Database, http://d0db.fnal.gov/qualitygrabber/ qualQueries.html.

[66] R. Demina, A. Khanov, Y. Kulik, A. Nomerotski, L. Shabalina, "Charge Distribution in SMT Clusters", DØNote 3981.

[67] G. Steinbruck, "Noise Studies with Silicon Microstrip Detectors", DØNote 3379.

[68] The DØ Trigger Board, http://www-d0.fnal.gov/RunIIaOperations/ TriggerBoard/.

[69] M. Michaut, B. Tuchming, "L2 Multijet Trigger Study for the hbb Channel", DØNote 4345.

[70] T. Sjostrand, S. Mrenna, P. Skands, "Pythia 6.5 Physics and Manual", HEP$\mathrm{PH} / 0603175$

[71] A. Haas, A. Kharchilava, M. Michaut, J. Rani, T. Scanlon, B. Tuchming, "D $\varnothing$ Search for Neutral Higgs Bosons at High $\tan \beta$ in Multi-jet Events Using p14 Data", DØNote 4671. 
[72] G. Bernardi, DØ Convenors Meeting, "Talk Higgs Status and Plans for Summer", May 2006.

[73] T. Scanlon, "A Neural Network b-tagging Tool”, DØNote 4889.

[74] T. Scanlon, M. Anastasoaie, "Performance of the NN b-tagging Tool on Pass2 Data", DØNote 4890.

[75] b-id Certification Guidelines, http://www-d0.fnal.gov/phys_id/bid/d0_ private/certification/p14Pass2/togetcert.html.

[76] D. Bloch, B. Clément, "Update of the JLIP b-tagger Performance in p14/pass2 with JES 5.3", DØNote 4824.

[77] D. Boline, L. Feligioni, M. Narain "Update on b-quark Jet Identification with Secondary Vertex Reconstruction using DØReco Version p14-Pass2 Samples", DØNote 4796.

[78] TMultiLayerPerceptron, http://root.cern.ch/root/html/ TMultiLayerPerceptron.html\#TMultiLayerPerceptron: description.

[79] MLPfit: a tool for Multi-Layer Perceptrons, http://schwind.home.cern.ch/ schwind/MLPfit.html.

[80] K. Hornik et al., "Multilayer Feedforward Networks are Universal Approximators", Neural Networks, Vol. 2, p359-366 (1989).

[81] D. Ruck et al., "The Multilayer Perceptron as an Approximation to a Bayes Optimal Discriminant Function", IEEE Transactions on Neural Networks, Vol. 1, p296-298 (1990).

[82] H. Robbins, S. Monro, "A Stochastic Approximation Method", Annals of Math. Stat. 22 (1951), p400.

[83] R.Fletcher, "Practical Methods of Optimization", second edition, Wiley (1987).

[84] Pass2 Skimmed Datasets, http://www-d0.fnal.gov/Run2Physics/cs/ skimming/pass2.html.

[85] M.L. Mangano, M. Moretti, F. Piccinini, R. Pittau, A. Polosa, "ALPGEN, a Generator for Hard Multiparton Processes in Hadronic Collisions", JHEP 0307:001,2003, hep-ph/0206293. 
[86] B. Clément, D. Bloch, D. Gelé, S. Greder, A.C. Le Bihan, I. Ripp-Baudot, "SystemD or how to get Signal, Backgrounds and their Efficiencies with Real Data", DØNote 4159.

[87] TMinuit, http://root.cern.ch/root/html/TMinuit.html.

[88] M. Spira, "Higgs boson Production and Decay at the Tevatron", hep$\mathrm{ph} / 9810289$.

[89] J. Vlimant, U. Bassler, G. Bernardi, S. Trincz-David, "Technical Description of the T42 Algorithm for the Calorimeter Noise Suppression", D $\varnothing$ Note 4146.

[90] M. Ford, S. Kappler, "Data Quality Report", All DØMeeting, May 2006.

[91] The Top Physics Working Group, "DØ Top Analysis and Data Sample for the Winter Conferences 2004", DØNote 4419.

[92] J. Rani, "Jet Efficiency Studies in $\gamma+$ jet Events: Data vs. Monte Carlo", DØNote 4363.

[93] A. Belyaev, E. Boos, "Single Top Quark tW+X Production at the LHC: A Closer Look", hep-ph/0003260, Phys.Rev. D63 (2001) 034012.

[94] J. Campbell, S. Dawson, S. Dittmaier, C. Jackson, M. Krämer, F. Maltoni, L. Reina, M. Spira, D. Wackeroth, S. Willenbrock, "Higgs Boson Production in Association with Bottom Quark", hep-ph/0405302 v1, 31 May, 2004.

[95] S. Dittmaier, M. Krämer, M. Spira, "Higgs Radiation off Bottom Quarks at the Tevatron and the LHC", Phys. Rev. D 70, 074010 (2004).

[96] J. Campbell, R.K. Ellis, F. Maltoni, S. Willenbrock, "Higgs-Boson Production in Association with a Single Bottom Quark", Phys.Rev.D67 (2003) 095002.

[97] S. Dawson, C. Jackson, L. Reina and D. Wackeroth, "Exclusive Higgs Production with Bottom Quarks at Hadron Colliders", Phys. Rev. D 69 (2004) 074027.

[98] CTEQ Collaboration, http://www.phys.psu.edu/ cteq/.

[99] The DØ Collaboration, "Combined $t \bar{t}$ Production Cross Section at $\sqrt{s}=1.96$ $\mathrm{TeV}$ in the Lepton+Jets and Dilepton Final States using Event Topology", DØNote 4906. 
[100] E. Nurse, P. Telford, "Measurement of $\sigma \times \mathrm{BR}$ for $\mathrm{Z} \rightarrow \mu^{+} \mu^{-}$in $p \bar{p}$ Collisions at $\sqrt{s}=1.96 \mathrm{TeV} "$, DØNote 4231 .

[101] F. Maltoni, T. Stelzer, "MADEVENT: Automatic Event Generation with MADGRAPH", hep-ph/0208156.

[102] J. Campbell, R. K. Ellis, F. Maltoni, S. Willenbrock, "Associated Production of a Z Boson and a Single Heavy-Quark Jet", hep-ph/0312024.

[103] A. Haas, "Level 1 and Level 2 Calorimeter Trigger Performance in Multi-jet Events", DØNote 4073.

[104] DØ Trigger Simulation, http://www-d0.fnal.gov/computing/trigsim/ trigsim.html.

[105] TLimit, http://root.cern.ch/root/html/TLimit.html.

[106] T. Junk, "Confidence Level Computation for Combining Searches with Small Statistics", Nucl. Instrum. Meth. A434 (1999) 435-443.

[107] The DØ Luminosity Group, "Luminosity Measurement", DØNote 4328.

[108] I. Iashvili, N. Parua, "Jet Energy Scale and Resolution for p14 Data and Monte Carl", http://www-d0.fnal.gov/phys\_id/jes/d0\_private/ certified/v5.0/links.html.

[109] L. Feligioni, M. Narain, P. Schieferdecker, A. Schwartzman "Update on bquark Jet Identification with Secondary Vertex Reconstruction using DØReco version p14", DØNote 4414.

[110] A. Pukhov et al., "COMPHEP", hep-ph/9908288.

[111] JES Group, "Jet Energy Scale and Resolution for Pass2 Fixed p14 Data and MC, with and without T42", http://www-d0.fnal.gov/phys \_id/jes/d0 \ _private/certified/v5.3/links.html.

[112] J. Heinmiller, N. Varelas, "Jet Reconstruction Efficiency", DØNote 4837.

[113] M. Anastasoaie, S. Robinson, T. Scanlon, "Performance of the NN b-tagging Tool on p17 Data", DØNote 5213. 